\title{
Multilagenbasierte Transmissionsoptiken \\ für die Röntgenmikroskopie
}

\author{
Dissertation \\ zur Erlangung des mathematisch-naturwissenschaftlichen Doktorgrades \\ „Doctor rerum naturalium“ \\ der Georg-August-Universität Göttingen
}

vorgelegt von

Tobias Liese

aus Rotenburg a. d. Fulda

Göttingen 2012 
Referent: Prof. Dr. H.-U. Krebs

Korreferentin: Prof. Dr. S. Köster

Tag der mündlichen Prüfung: 15.05.2012 


\section{Inhaltsverzeichnis}

1 Einleitung 1

2 Theoretische Grundlagen und aktueller Forschungsstand 5

2.1 Wechselwirkung von Röntgenstrahlung mit Materie . . . . . . . . . . 5

2.2 Konventionelle Zonenplatten . . . . . . . . . . . . . . . . . . 7

2.2.1 Beugungseffizienz . . . . . . . . . . . . . . . . . 11

2.2.2 Auflösungsvermögen . . . . . . . . . . . . . . . 16

2.2.3 Entwicklung der Zonenplatten-Herstellung . . . . . . . . . . . . 17

2.3 Multilagenbasierte Transmissionsoptiken . . . . . . . . . . . . . . . 21

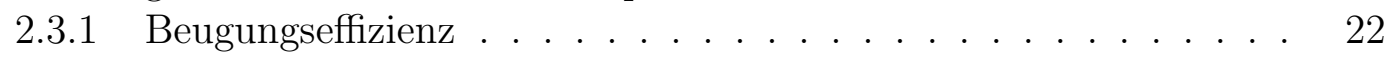

2.3.2 Auflösungsvermögen . . . . . . . . . . . . . . . . . . 25

2.3.3 Entwicklung der MLL- und MZP-Herstellung . . . . . . . . . 27

3 Experimentelle Methoden 31

3.1 Gepulste Laserdeposition . . . . . . . . . . . . . . . . . . . . . . . 31

3.2 Fokussierte Ionenstrahltechnik . . . . . . . . . . . . . . . . . . . 33

3.3 „table-top"-Röntgenmikroskopie . . . . . . . . . . . . . . . . . 34

3.4 Untersuchungen mit Synchrotronstrahlung . . . . . . . . . . . . . . . 35

3.5 Schichtdickenanalyse . . . . . . . . . . . . . . . . . 35

3.5.1 Profilometrie . . . . . . . . . . . . . . . 36

3.5 .2 Massenwägung . . . . . . . . . . . . . . . . 36

3.5.3 Röntgenreflektometrie . . . . . . . . . . . . . . . 37

3.5.4 Transmissionselektronenmikroskopie . . . . . . . . . . . . . 39

3.6 Strukturanalyse . . . . . . . . . . . . . . . . . . . . . . . 39

3.6.1 Rasterelektronenmikroskopie . . . . . . . . . . . . . . . 39

3.6.2 Röntgendiffraktometrie . . . . . . . . . . . . . . . . 39

3.6.3 EUV-Reflektometrie . . . . . . . . . . . . . . 40

4 Optimierung zur Herstellung hochpräziser Multischichtstrukturen $\quad 41$

4.1 Stabilisierung der Energiedichte am Target . . . . . . . . . . . . . . . . . . . . 42

4.1 .1 Energieregelung des Lasers . . . . . . . . . . . . . . . . . . 42

4.1.2 Verlängerung des Strahlengangs im Vakuum . . . . . . . . . . . 44

4.2 Homogenisierung des Targetabtrags . . . . . . . . . . . . . . . . . . . 46 
5 Transmissionsoptiken für weiche Röntgenstrahlung $\quad 49$

5.1 Teststruktur einer Multilagen-Laue-Linse . . . . . . . . . . . . . . . . . 49

5.1 .1 Systemauswahl und Design . . . . . . . . . . . . . . . 50

5.1.2 Charakteristik von laserdeponiertem $\mathrm{Ti}$ und $\mathrm{ZrO}_{2}$. . . . . . . 55

5.1.3 Untersuchungen am $\mathrm{Ti} / \mathrm{ZrO}_{2}-$ Multischichtsystem . . . . . . . . 63

5.1.4 MLL-Herstellung mittels PLD und FIB . . . . . . . . . . . 70

5.1.5 Strahlcharakteristik am „tabletop“-Mikroskop . . . . . . . . . 76

5.2 Weitere linear fokussierende Optiken . . . . . . . . . . . . . . 80

5.2.1 Herstellung einer dünnen $\mathrm{MgO} / \mathrm{ZrO}_{2}-\mathrm{MLL}$. . . . . . . . . . . . 81

5.2.2 Herstellung einer dicken $\mathrm{MgO} / \mathrm{ZrO}_{2}-\mathrm{MLL} \ldots \ldots$. . . . . . . . 88

5.2.3 Herstellung eines $\mathrm{Ti} / \mathrm{ZrO}_{2}-$ Röntgenmonochromators . . . . . . . 89

5.3 Laserdeponierte Multilagen-Zonenplatten . . . . . . . . . . . . . . . . . 93

5.3.1 Vorbereitende Beschichtungsprozesse . . . . . . . . . . . . 93

5.3.2 Beschichtung rotierender Drähte . . . . . . . . . . . . . 95

5.4 Zusammenfassung und Ausblick . . . . . . . . . . . . . . . . 100

6 Transmissionsoptiken für harte Röntgenstrahlung 103

6.1 Systemauswahl und Design von Multilagen-Laue-Linsen . . . . . . . . 103

6.2 Vorbereitende Untersuchungen am W/Si-System . . . . . . . . . . . 108

6.2.1 W- und Si-Einzelschichten . . . . . . . . . . . . . . . . 109

$6.2 .2 \mathrm{~W} / \mathrm{Si}-$ Multischichten . . . . . . . . . . . . . . . . . . 116

6.2.3 Theoretische Analysen auftretender Grenzflächen-Phänomene . 121

6.3 MLL-Schichtherstellung mittels PLD . . . . . . . . . . . . . . . . . 129

6.4 Eindimensionale MLL-Fabrikation mittels FIB . . . . . . . . . . . . . 137

6.5 Strahlcharakteristik am Synchrotron . . . . . . . . . . . . . . . 140

6.6 Design und Herstellung zweidimensionaler Multilagen-Laue-Linsen . 147

6.7 Ausblick . . . . . . . . . . . . . . . . . . . . 151

$\begin{array}{lll}7 & \text { Zusammenfassung } & 155\end{array}$

$\begin{array}{ll}\text { Literaturverzeichnis } & 176\end{array}$ 


\section{Einleitung}

Als Wilhelm Conrad RöNTGEN 1895 kurz nach seiner Entdeckung der Röntgenstrahlung zu der Erkenntnis kam, „dass man mit Linsen die X-Strahlen nicht concentriren kann" [1], konnte er nicht ahnen, welch intensiver Forschungsdrang entwickelt wurde, es schließlich doch zu tun. Dieser war vor allem durch den Wunsch geprägt, die Mikroskopie in den kurzwelligen Bereich der Röntgenstrahlung $(\lambda=0,001-10 \mathrm{~nm})$ auszudehnen, zumal die Auflösung eines Mikroskops wesentlich von der Wellenlänge des verwendeten Lichts bzw. der elektromagnetischen Strahlung abhängt [2]. Mittlerweile hat sich die Röntgenmikroskopie als komplementäres Bindeglied zwischen der Lichtund der Elektronenmikroskopie etabliert [3] (s. Abb. 1.1). Die Vorteile sind in einer verbesserten räumlichen Aufösung gegenüber der konventionellen Lichtmikroskopie und einer größeren Eindringtiefe bei einfacherer Probenpräparation gegenüber der Elektronenmikroskopie zu sehen. Lag der damalige Forschungsschwerpunkt hauptsächlich in der kontrastreichen Abbildung biologischer Zellen im weichen Röntgenwellenlängenbereich des sog. „Wasserfensters“ $(2,3-4,4 \mathrm{~nm})$, ist heute außerdem eine Vielzahl moderner Applikationen wie z. B. Fluoreszenz-Röntgenmikroskopie, Spektromikroskopie, kohärente Röntgenbeugung zur linsenlosen Abbildung, PhasenkontrastRöntgenmikroskopie und 3D-Röntgentomographie mit weicher $(0,5-6 \mathrm{~nm})$ sowie harter Röntgenstrahlung $(<0,5 \mathrm{~nm})$ in der Medizin, Biophysik, Materialwissenschaft, Umweltphysik, Archäologie, Paläontologie etc. fest verankert [4-12].

Der einhergehende rasante Fortschritt von hochbrillanten Synchrotronstrahlungsquellen [15] aber auch die zunehmende Entwicklung von kompakten labortauglichen Röntgenmikroskopen [16] verlangt nach immer besseren optischen Elementen zur Strahlfokussierung, durch welche die erreichbare Auflösung wesentlich beschränkt ist. Die Arten von optischen Linsen im Röntgenbereich gleichen allerdings nicht derer, die für sichtbares Licht funktionieren. Der Brechungsindex energetischer Röntgenstrahlung ist für alle Materialien ungefähr gleich 1, sodass mit einer herkömmlichen Brechungslinse keine ausreichende Wechselwirkung zur Strahlmanipulation besteht. Als fokussierende Optiken finden daher hauptsächlich reflektive Kirkpatrick-Baez- 


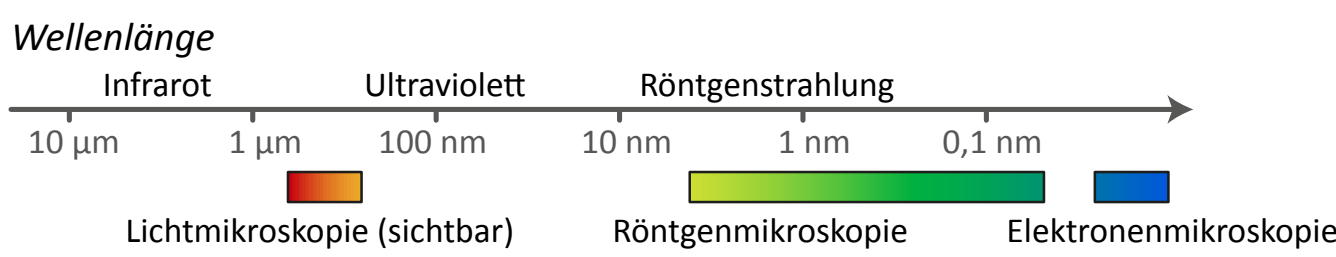

Objektgröße

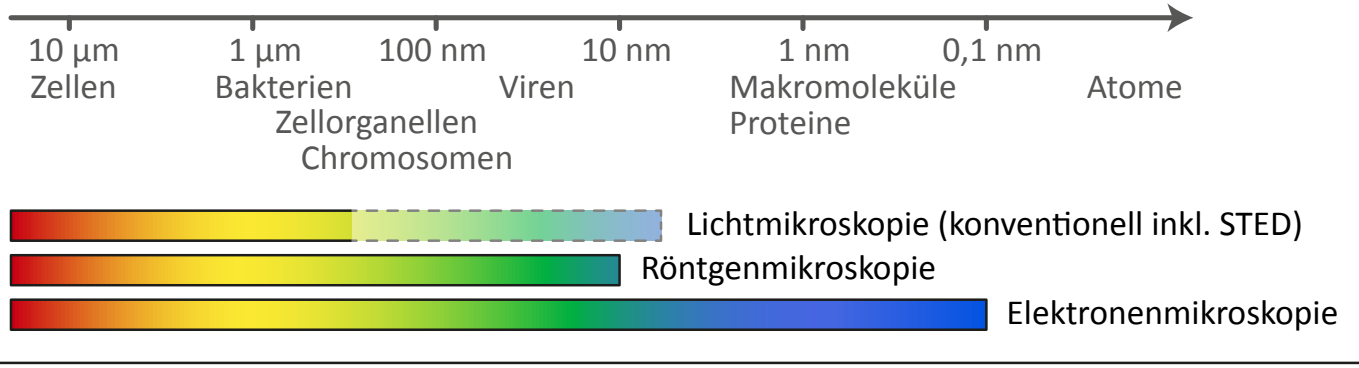

Abbildung 1.1: Übersicht der verwendeten Wellenlängen in der Mikroskopie und deren Einsatzbereiche (nach [3], angepasst durch aktuellen Forschungsstand). Mittels einer speziellen Fluoreszenz-Technik (engl. stimulated emission depletion, STED) gelang es in der Lichtmikroskopie, die natürliche Auflösungsgrenze von ungefähr $200 \mathrm{~nm}$ (Abbe'sche Theorie) zu überwinden [13]. Heute werden damit Auflösungen von ca. 5-6 nm erreicht [14].

Spiegel [17, 18], reflektive Wellenleiter [19, 20], refraktive Verbundlinsen [21,22] und diffraktive Zonenplatten [23-26] Verwendung. Mit einem Röntgenmikroskop auf Zonenplattenbasis konnte zu Beginn der 80er Jahre die Aufösungsgrenze der Lichtmikroskopie (ca. $200 \mathrm{~nm}$ ) von Schmahl et al. zum ersten Mal überwunden werden $[27,28]$.

Zonenplatten sind radialsymmetrische Kreisgitter mit nach außen abnehmender Gitterkonstante, deren maximale Aufösung in der Größenordnung der Breite der äußersten Zone liegt [29]. Die bislang am erfolgreichsten angewandte Fertigungsmethode der Elektronenstrahl-Lithographie birgt jedoch zwei wesentliche Herstellungsprobleme: Zum einen ist es besonders kompliziert, auflösungsbestimmende äußere Zonenbreiten kleiner als $10 \mathrm{~nm}$ herzustellen und zum anderen ist es enorm schwierig, optische Zonentiefen größer als $100 \mathrm{~nm}$ zu erzeugen, die den Beugungswirkungsgrad einer Zonenplatte erheblich beeinflussen können [30]. Eine vielversprechende Alternative stellt die Fabrikation von ein- oder zweidimensionalen Zonenplattenstrukturen (Linienoder Punktfokus) auf der Basis aperiodischer Multischichten dar [31]. Mit sog. Multilagen-Laue-Linsen (MLL, 1D) oder Multilagen-Zonenplatten (MZP, 2D) lassen sich effektive Strahlfokussierungen durch hohe Aspektverhältnisse (von Zonentiefe zu Zonenbreite) bewerkstelligen. 
Das Ziel der vorliegenden Arbeit ist die Entwicklung und Optimierung einer neuartigen Herstellungsmethode multilagenbasierter Transmissionsoptiken für den weichen und harten Röntgenwellenlängenbereich, welche die Verwendung zweier komplementärer Methoden umfasst: die gepulste Laserdeposition und die fokussierte Ionenstrahltechnik. Im Vordergrund steht dabei ein elementares physikalisches Verständnis von gezieltem Schichtwachstum und realer Grenzflächeneigenschaften geeigneter Materialsysteme sowie eine defektfreie und unkomplizierte Fabrikation entsprechender Optiken zur effizienten Fokussierung von Röntgenstrahlung mit möglichst hoher Auflösung im Bereich unter $10 \mathrm{~nm}$.

Inhaltlich ist die Arbeit wie folgt gegliedert: Zunächst werden in Kapitel 2 theoretische Betrachtungen der Beugungstheorie von konventionellen Zonenplatten und alternativen multilagenbasierten Transmissionsoptiken bezüglich ihrer maximal möglichen Effizienz und Auflösung mit jeweils anschließendem aktuellen Forschungsstand aufgeführt. Nachdem in Kapitel 3 angewandte experimentelle Methoden vorgestellt werden, folgt in Kapitel 4 ein Auszug notwendiger Prozessoptimierungen der gepulsten Laserdeposition. Die dadurch erst ermöglichte Herstellung aperiodischer Multischichten und die Fabrikation von MLL sowie MZP unterschiedlicher Geometrien und Materialsysteme für weiche Röntgenstrahlung sind in Kapitel 5 detailliert beschrieben. Eindimensionale MLL im weichen Röntgenwellenlängenbereich sind in der Literatur bisher nicht bekannt. Kapitel 6 beinhaltet schließlich die Realisierung einund zweidimensionaler MLL für harte Röntgenstrahlung. Hauptaugenmerk wird dabei auf die Untersuchung der Grenzflächeneigenschaften des benutzten Materialsystems zur Herstellung hochpräziser aperiodischer Multischichten gelegt. 



\section{Theoretische Grundlagen und aktueller Forschungsstand}

Im folgenden Kapitel sollen zunächst die Wechselwirkung von Röntgenstrahlung mit Materie, die Beugungstheorie von konventionellen Zonenplatten und deren technologische Herstellungsgrenzen behandelt werden. Alternative Methoden zur Erzeugung multilagenbasierter ein- und zweidimensionaler Zonenplattenstrukturen (Multilagen-Laue-Linsen und Multilagen-Zonenplatten) werden im Anschluss theoretisch beschrieben sowie experimentelle Vor- und Nachteile aufgezeigt.

\subsection{Wechselwirkung von Röntgenstrahlung mit Materie}

Die Wechselwirkung von Röntgenstrahlung mit Materie hat eine Phasenverschiebung $\delta$ der einfallenden Welle und eine Dämpfung $\beta$ ihrer Amplitude zur Folge und wird zusammenfassend im komplexen Brechungsindex $n$ eines Mediums formuliert [32]:

$$
n=1-\delta-\mathrm{i} \beta
$$

Wird die Bestrahlung einer Materialschicht der Dicke $\Delta t$ mit einer ebenen Welle der Amplitude $A_{0}$ und einer festen Wellenlänge $\lambda$ betrachtet, ergibt sich mit der Amplitude $A_{1}$ der hindurchtretenden Welle der Transmissionskoeffizient $T$ zu

$$
T=\frac{A_{1}}{A_{0}}=e^{-\frac{2 \pi}{\lambda} \beta \Delta t} e^{-\mathrm{i} \frac{2 \pi}{\lambda}(1-\delta) \Delta t}
$$

Der erste Term beschreibt die Dämpfung durch photoelektrische Absorption und der zweite die Phasenverschiebung der transmittierenden Welle. Die durch Absorption 
resultierende Abschwächung der eingestrahlten Intensität $I_{0}=A_{0} A_{0}^{*}$ zu $I_{1}=A_{1} A_{1}^{*}$ beträgt

$$
\frac{I_{1}}{I_{0}}=e^{-4 \pi \beta \Delta t / \lambda}
$$

Dabei stellt die Größe $\mu_{l}=4 \pi \beta / \lambda$ den linearen Absorptionskoeffizienten dar. Auf atomarer Ebene können die Phasenverschiebung und die Absorption durch den komplexen atomaren Streufaktor $f=f_{1}+\mathrm{i} f_{2}$ ausgedrückt werden. In der Annahme einer Vorwärtsstreuung $(\theta=0)$ lässt sich mittels der atomaren Dichte $n_{a}$ und dem klassischen Elektronenradius $r_{e}$ ein Zusammenhang zwischen Brechungsindex und Streufaktor herstellen [33]:

$$
n=1-\frac{n_{a} r_{e} \lambda^{2}}{2 \pi}\left(f_{1}+\mathrm{i} f_{2}\right)
$$

Die Variation der Phase wird dabei durch $f_{1}$ und die Dämpfung der Wellenamplitude aufgrund von Absorption durch $f_{2}$ berücksichtigt. Im Vergleich zwischen Formel 2.1 und 2.4 lässt sich die Phasenverschiebung und die Absorption in folgender Form ermitteln:

$$
\delta=\frac{n_{a} r_{e} \lambda^{2}}{2 \pi} f_{1} \quad \text { und } \quad \beta=\frac{n_{a} r_{e} \lambda^{2}}{2 \pi} f_{2}
$$

Im Bereich weicher und harter Röntgenstrahlung nehmen $\delta$ und $\beta$ sehr geringe Werte von ungefähr $10^{-7}-10^{-2}$ an. Der komplexe Brechungsindex weicht somit kaum von $1 \mathrm{ab}$, sodass lediglich eine schwache Wechselwirkung zwischen hochenergetischer Strahlung und Materie besteht. Zur Strahlfokussierung im Röntgenbereich ist daher die Verwendung herkömmlicher Brechungslinsen kaum lohnenswert. Bei weichen Wellenlängen wäre die Absorption viel zu hoch und bei harter Röntgenstrahlung würden sich aufgrund der geringen Wechselwirkung nur sehr hohe Brennweiten und somit geringe Auflösungen ergeben. Letzteres würde sich durch die Anordnung vieler bikonkaver Einzellinsen (engl. compound refractive lens) umgehen lassen [21].

Neben zahlreichen anderen Methoden zur Strahlfokussierung ist die Verwendung von Fresnelschen Zonenplatten, die anstelle der Brechung das Prinzip der Beugung ausnutzen, von großem Interesse [33-35]. Bereits 20 Jahre vor der Entdeckung der Röntgenstrahlung sind 1875 deren beugenden Eigenschaften im sichtbaren Wellenlängenbereich von SoRET beschrieben worden [36], welcher daher als Entdecker dieses 
Phänomens gilt. Die früheste Aufzeichnung über die mögliche Fokussierung des Lichts mit undurchlässigen Fresnel-Zonen, welche allerdings erst 1889 veröffentlicht wurde, stammt aus dem Jahr 1871 von LoRD RAYLEIGH [37].

\subsection{Konventionelle Zonenplatten}

Zonenplatten (ZP) sind radialsymmetrische Kreisgitter, die im einfachsten Fall aus abwechselnd transparenten und absorbierenden Zonen bestehen. Genau wie beim periodischen Gitter ist der Beugungswinkel bei fester Wellenlänge abhängig vom Abstand der einzelnen Zonen $d$. Soll die einfallende Strahlung in einem Brennpunkt der Ordnung $m$ fokussiert werden, müssen die Abstände der Zonen bei größer werdendem Beugungswinkel gemäß der Beziehung

$$
\sin \theta_{m}=\frac{m \lambda}{d}
$$

von der optischen Achse aus nach außen hin abnehmen (vgl. Abb. 2.1), wobei jede Zone die gleiche Fläche wie alle anderen besitzt und gleichermaßen zu einer Fokussierung beiträgt. Sind die Weglängen durch die jeweils transparenten Zonen um $\lambda$ verschieden, kommt es am Ort des Fokus im Abstand der Brennweite (oder Fokuslänge) $f \mathrm{zu}$ phasengleicher, konstruktiver Interferenz. Die Einzelamplituden dieser Beugungsordnung addieren sich zu einem Intensitätsmaximum auf, welches sich aufgrund der konzentrischen Lage des Gitters auf der optischen Achse befindet. Ob nun die geraden oder ungeraden Zonen transparent sind, spielt für die gebeugte Intensität im Fokus keine Rolle, es tritt lediglich ein Phasenschub um $\lambda / 2$ auf. Da die Bedingung für konstruktive Interferenz für verschiedene Richtungen erfüllt ist, existieren neben dem ungebeugten Nullstrahl weitere Beugungserscheinungen höherer Ordnung. Beträgt der Weglängenunterschied das $m$-fache der Wellenlänge, tritt bei verringerter Fokuslänge ( $\widehat{=}$ größerem Beugungswinkel) die $m$-te Beugungsordnung auf. Der am weitesten von der ZP entfernte Brennpunkt gehört zur 1. Beugungsordnung, der nächstnähere wird Brennpunkt 3. Ordnung usw. genannt. Gerade Beugungsordnungen $(m= \pm 2, \pm 4 \ldots)$ treten nur dann durch teilweise konstruktive Interferenz auf, wenn aufeinander folgende Flächen von transparenten und absorbierenden Zonen nicht gleich groß sind. Bei einer idealen ZP werden sie jedoch durch destruktive Interferenz am Ort des Fokus ausgeblendet. Im Vergleich zur herkömmlichen Linse existiert bei einer ZP also eine Vielzahl an Brennpunkten. 


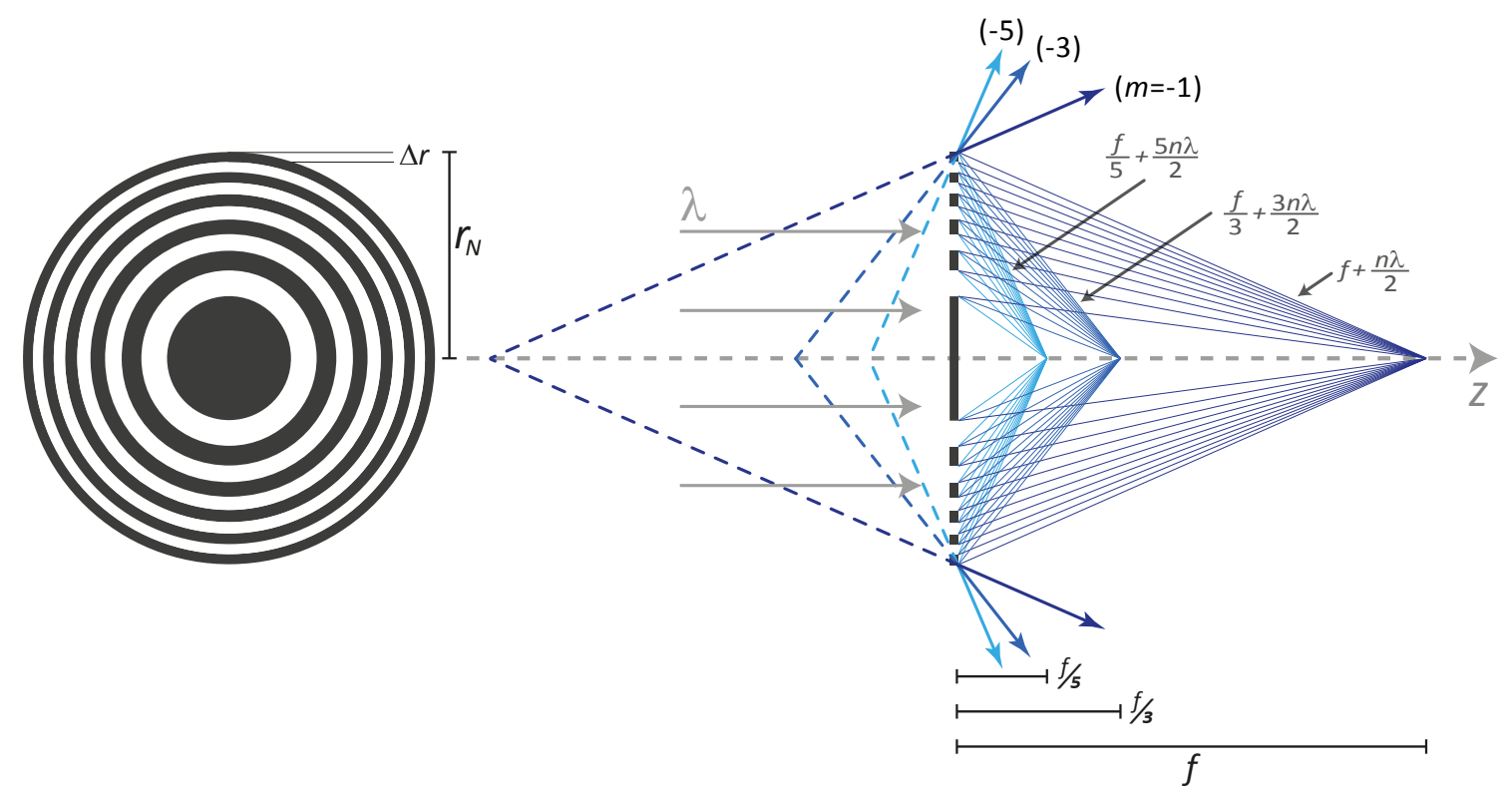

Abbildung 2.1: Front- und Seitenansicht einer Fresnelschen Zonenplatte. Nur wenn der Weglängenunterschied der Teilstrahlen ein Vielfaches der Wellenlänge beträgt, findet am Ort des Fokus konstruktive Interferenz statt. Die Zonenbreite muss dabei nach außen hin sukzessiv abnehmen. Neben der am weitesten entfernten +1 . Beugungsordnung mit der Fokuslänge $f$ sind weitere höhere Ordnungen dargestellt (nach [33]).

Wichtige Kenngrößen wie die Fokuslänge $f$, die Weglänge $f+n \lambda / 2$ durch die Zone $n$ sowie deren Radius $r_{n}$ werden über das Gesetz von Pythagoras als

$$
f^{2}+r_{n}^{2}=\left(f+\frac{n \lambda}{2}\right)^{2}
$$

zusammengefasst. Durch einfache Umwandlung wird das binäre Zonenplattenbildungsgesetz, welches den jeweiligen Zonenabstand zur optischen Achse beschreibt, hergeleitet [33].

$$
r_{n}=\sqrt{n \lambda f+\frac{n^{2} \lambda^{2}}{4}}
$$

Der quadratische Term, welcher die sphärische Aberration dieser Transmissionsoptik berücksichtigt, kann für $f \gg n \lambda / 2$ vernachlässigt werden, sodass das Zonenplattenbildungsgesetz in vereinfachter Form zu

$$
r_{n} \simeq \sqrt{n \lambda f}
$$


genähert werden kann. Sollen alle transmittierten Teilstrahlen einer ZP schließlich einen gemeinsamen Fokus besitzen, muss die Zonenperiode sukzessive um $\sqrt{n}$ abnehmen.

Wird nun konkret eine ZP mit $N$ Zonen, dem Durchmesser $D=2 r N$ und der Fokuslänge $f$ betrachtet, so ist die äußere Zonenbreite $\Delta r$ durch

$$
\Delta r \equiv r_{N}-r_{N-1}
$$

gegeben. Werden beide Radien mittels des genäherten Zonenplattenbildungsgesetzes formuliert, ergibt sich nach ihrer Subtraktion die Beziehung

$$
r_{N}^{2}-r_{N-1}^{2}=N \lambda f-(N-1) \lambda f=\lambda f
$$

wobei sich die linke Seite gemäß der Definition von $\Delta r$ separat mit

$$
r_{N}^{2}-\left(r_{N}-\Delta r\right)^{2}=2 r_{N} \Delta r-(\Delta r)^{2} \simeq 2 r_{N} \Delta r
$$

behandeln lässt, sofern $\Delta r \ll r_{N}$ für große $N$ ist. Die Kombination beider Gleichungen führt schließlich zu

$$
2 r_{N} \Delta r \simeq \lambda f \quad \text { oder } \quad D \Delta r \simeq \lambda f
$$

Wiederum lässt sich $\lambda f$ mit Formel 2.9 näherungsweise durch $r_{N}^{2} / N$ umschreiben, sodass mittels $D=2 r_{N} N$ der Durchmesser einer ZP allein mit der Gesamtzahl aller Zonen und der kleinsten Zonenbreite in Beziehung gesetzt wird.

$$
D \simeq 4 N \Delta r
$$

Durch Superposition von Gleichung 2.11 und 2.12 wird schließlich ein Ausdruck für die Fokuslänge $f$ bezogen.

$$
f \simeq \frac{4 N(\Delta r)^{2}}{\lambda}
$$


Mit $f \sim 1 / \lambda$ ist der Ort des Brennpunktes einer Beugungsordnung demnach stark wellenlängenabhängig, sodass ZP in bestimmten Geometrien auch als Röntgenmonochromatoren (s. Kap. 5.2.3) eingesetzt werden können.

Zusätzlich soll die numerische Apertur $N A$ eingeführt werden, ein weiterer Parameter, der für die Definition der Aufösung einer ZP benötigt wird (s. Kap. 2.2.2).

$$
N A \equiv \sin \theta=\frac{D}{2 f}
$$

Der Winkel $\theta$ entspricht hier dem halben Öffnungswinkel einer ZP. Mit $D \simeq \lambda f / \Delta r$ (s. Gl. 2.11) kann die numerische Apertur ebenfalls durch die äußere Zonenbreite $\Delta r$ beschrieben werden.

$$
N A \simeq \frac{\lambda}{2 \Delta r}
$$

Fresnelsche Zonenplatten, die nach dem Bildungsgesetz hergestellt werden, können auf zwei verschiedene Weisen in einem Röntgenmikroskop eingesetzt werden [32]:

Bei Vollfeld-Röntgenmikroskopen z. B. befinden sich Kondensor-ZP, die mit einer sehr großen Anzahl an Zonen mehrere Millimeter im Durchmesser einnehmen, zwischen Röntgenquelle und zu beleuchtendem Objekt und funktionieren in Verbindung mit einer Lochblende als monochromatisierende Sammellinse (s. Abb. 2.2 oben). Der Beugungswirkungsgrad, also das Verhältnis zwischen eingestrahlter und gebeugter Intensität, sollte dabei möglichst hoch sein, um einen hohen Anteil der aufgefangenen Strahlung auf das Objekt zu fokussieren. Eine zentrale hinter der Kondensor-ZP befindliche Blende verhindert die Objektbeleuchtung mit ungebeugter Röntgenstrahlung 0. Ordnung. Zwischen Objekt und Detektor befindet sich eine Mikro-ZP zur hochauflösenden Bildgebung.

Bei Raster-Röntgenmikroskopen wird die Mikro-ZP entsprechend vor der Probe positioniert, um lokale analytische Untersuchungen durchzuführen oder aus den Intensitätsverteilungen einzelner abgerasterter Flächensegmente ein vollständiges, vergröBertes Probenabbild zu erzeugen (s. Abb. 2.2 unten). Zur Abrasterung kann entweder das Objekt oder die ZP verfahren werden. Auch hier dient die zentrale Blende zur Abschattung der 0. Beugungsordnung. 

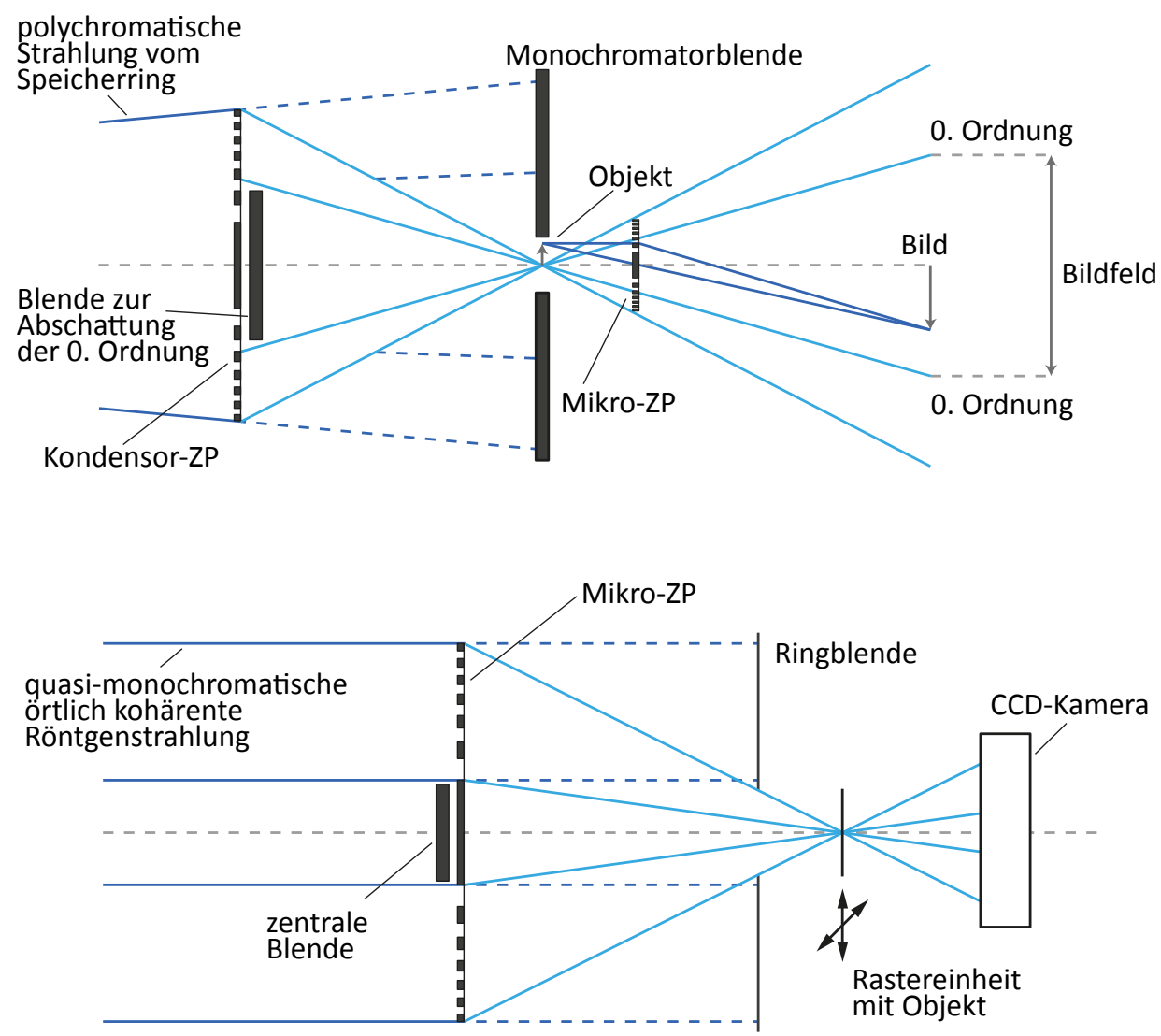

Abbildung 2.2: Strahlengang eines Vollfeld- und eines Raster-Röntgenmikroskops. Während bei einem Vollfeldmikroskop (oben) Kondensor- und Mikro-ZP zur Bildgebung benutzt werden, wird beim Raster-Röntgenmikroskop (unten) mit vorausgesetzter kleiner Röntgenquelle lediglich eine MikroZP verwendet (nach [32]).

\subsubsection{Beugungseffizienz}

Sowohl für Kondensor- als auch Mikro-ZP spielt deren Qualität für die Anwendung in der Röntgenmikroskopie eine zentrale Rolle. Durch Eigenschaften wie maximal erreichbare Effizienz und bestmögliche Auflösung wird die Charakteristik der ZP vollständig beschrieben. Bezüglich der maximalen Effizienz lassen sich im Wesentlichen drei Klassen von ZP betrachten: (a) Absorptions-- bzw. Amplitudenzonenplatten (Fresnel), (b) binäre phasenschiebende Zonenplatten (Rayleigh-Wood) und (c) mehrstufige phasenschiebende Zonenplatten (Kinoform).

(a) Bei einer vollständig absorbierenden, dünnen Fresnelschen ZP (Aspektverhältnis von Zonentiefe zu Zonenbreite kleiner als 10:1) kommt es anhand der undurchlässigen Zonen zu einer Ausblendung derjenigen Elementarwellen, die sonst mit gegenläufiger Phase destruktiv zum Wellenfeld beitragen würden. Mit einem großen Verhältnis 
von $k=\beta / \delta$ dominiert hierbei die Absorption des Zonenmaterials, sodass $50 \%$ der einfallenden Strahlung absorbiert werden und weitere $25 \%$ ungebeugt transmittieren. Die restlichen $25 \%$ verteilen sich auf die ungeraden Beugungsordnungen, bei denen in geometrischer Näherung betrachtet die Effizienz $\eta=I_{1} / I_{0}$ mit dem Quadrat der Beugungsordnung $m$ deutlich abfällt [38]: (Diese Formel ergibt sich als Spezialfall von Gl. 2.18 mit hoher Absorption bzw. großem $\beta$.)

$$
\eta=\frac{1}{(\pi m)^{2}}
$$

In der Mikroskopie wird daher hauptsächlich in der 1. Beugungsordnung gearbeitet, in die ca. $10 \%$ der transmittierten Strahlung fokussiert wird. Die für ein Zonenmaterial benötigte optische Tiefe für nahezu vollständige Absorption lässt sich anhand der Intensitätsabschwächung innerhalb des Materials anhand Gleichung 2.3 für $I_{1} \rightarrow 0$ abschätzen. Je größer hierbei die Absorption des Zonenmaterials ist, desto geringer ist die herzustellende optische Tiefe einer ZP.

(b) Sind $\beta$ und $\delta$ nicht stark unterschiedlich, können durch die Wahl eines teiltransparenten Zonenmaterials weitaus höhere Effizienzen erzielt werden (RayleighWood), indem sich bei geeigneter Zonentiefe $\Delta t$ ein Phasenschub um $\Delta \phi=\pi$ bezüglich der durch die transparenten Zonen transmittierten Strahlung einstellt [39].

$$
\Delta \phi=\frac{2 \pi \delta \Delta t}{\lambda}
$$

Bei einer solchen Phasendifferenz interferieren alle durch die Zonen hindurchtretenden Teilstrahlen konstruktiv, sodass sich theoretisch bei verdoppelter Amplitude für jede Beugungsordnung eine vierfache Intensität ergibt (ca. 40\% in der 1. Beugungsordnung).

Mit einer idealen Phasen-ZP $(k \rightarrow 0)$ ließe sich die 0. Beugungsordnung unter Vernachlässigung der Absorption sogar vollständig unterdrücken. Die Möglichkeit, eine phasenschiebende ZP zu realisieren, hängt dabei stark von der Herstellungsmethode, dem Absorptionsgrad des Zonenmaterials und der verwendeten Wellenlänge ab. Gerade bei weicher Röntgenstrahlung nimmt die hohe Absorption innerhalb der Zonen einen großen Einfluss auf die zu erwartende Effizienz, sodass KIRZ in die Berechnung der Intensitäten verschiedener Beugungsordnungen nicht nur die Phasenmodulation, sondern auch die Absorption innerhalb des verwendeten Materials (vgl. Formel 2.3) 
mit einfließen lässt [39]. Für weiche Wellenlängen werden Gleichung 2.16 weitere Terme hinzugefügt:

$$
\eta=\frac{1}{(\pi m)^{2}}\left(1+e^{-4 \pi \beta \Delta t / \lambda}-2 e^{-\pi \beta \Delta t / \lambda} \cos (2 \pi \delta \Delta t / \lambda)\right)
$$

Abbildung 2.3 zeigt dazu die in 1. Beugungsordnung theoretisch erreichbaren Effizienzen $\eta$ für einige typische Materialien zur Herstellung von ZP für weiche Röntgenstrahlung bei fester Wellenlänge $\lambda=2,88 \mathrm{~nm}$ auf. Hier wird sichtbar, wie sich der Phasen-Effekt für verschiedene Materialien in Abhängigkeit der optischen Tiefe $\Delta t$ äußert. Im direkten Vergleich zu Nickel und Germanium weist Beryllium den kleinsten $k$-Wert von 0,15 und daher die größtmögliche Beugungseffizienz von $33 \%$ auf. Um allerdings bei einer hochaufösenden ZP den dafür notwendigen Phasenschub von $\pi$ zu erzeugen, wäre in diesem Fall eine hohe und somit für die LithographieTechnik (s. Kap. 2.2.3) kaum zu realisierende optische Tiefe von $670 \mathrm{~nm}$ erforderlich. Nickel und Germanium besitzen vergleichsweise hohe $k$-Werte von 0,25 bzw. 0,46, sodass die berechneten Effizienzen zwar etwas schwächer sind, dafür aber aufgrund größerer Phasenmodulation wesentlich kleinere optische Tiefen von nur 200 bzw. $320 \mathrm{~nm}$ notwendig wären. Abgesehen von Herstellungsproblemen ist es also bzgl. einer maximalen Effizienz generell von Vorteil, diejenigen Zonenmaterialien zu verwenden, die bei bestimmter Wellenlänge über einen kleinen $k$-Wert verfügen.

(c) Mit komplizierten, mehrstufigen phasenschiebenden ZP (Multilevel-ZP) ist es theoretisch möglich, annähernd 100\% der einfallenden Intensität in der 1. Beugungsordnung zu fokussieren. Dies geschieht nicht wie bei einer binären Rayleigh-WoodZP mit einem abrupten Phasenschub von 0 auf $\pi$ zwischen transmittierender und offener Zone, sondern mit einer kontinuierlichen Phasenmodulation (Kinoform), indem jede teiltransparente Zone nach folgendem modifizierten Zonenplattenbildungsgesetz durch beliebig viele Unterzonen (Phasenlevel) mit einem parabolischen Tiefenprofil in $z$-Richtung dargestellt wird [41]:

$$
r_{n, L}=\sqrt{\lambda f\left(2 \frac{l}{L}+n-2\right)} .
$$

Jede Zone mit einem geraden Zonenindex ist hierbei in $L$ Phasenlevel mit $1 \leq l \leq L$ eingeteilt, wobei sich die Formel für $L=2$ wiederum auf das binäre Zonenplattenbildungsgesetz (s. Gl. 2.9) reduzieren lässt. Innerhalb jeder Levelzone steigt 


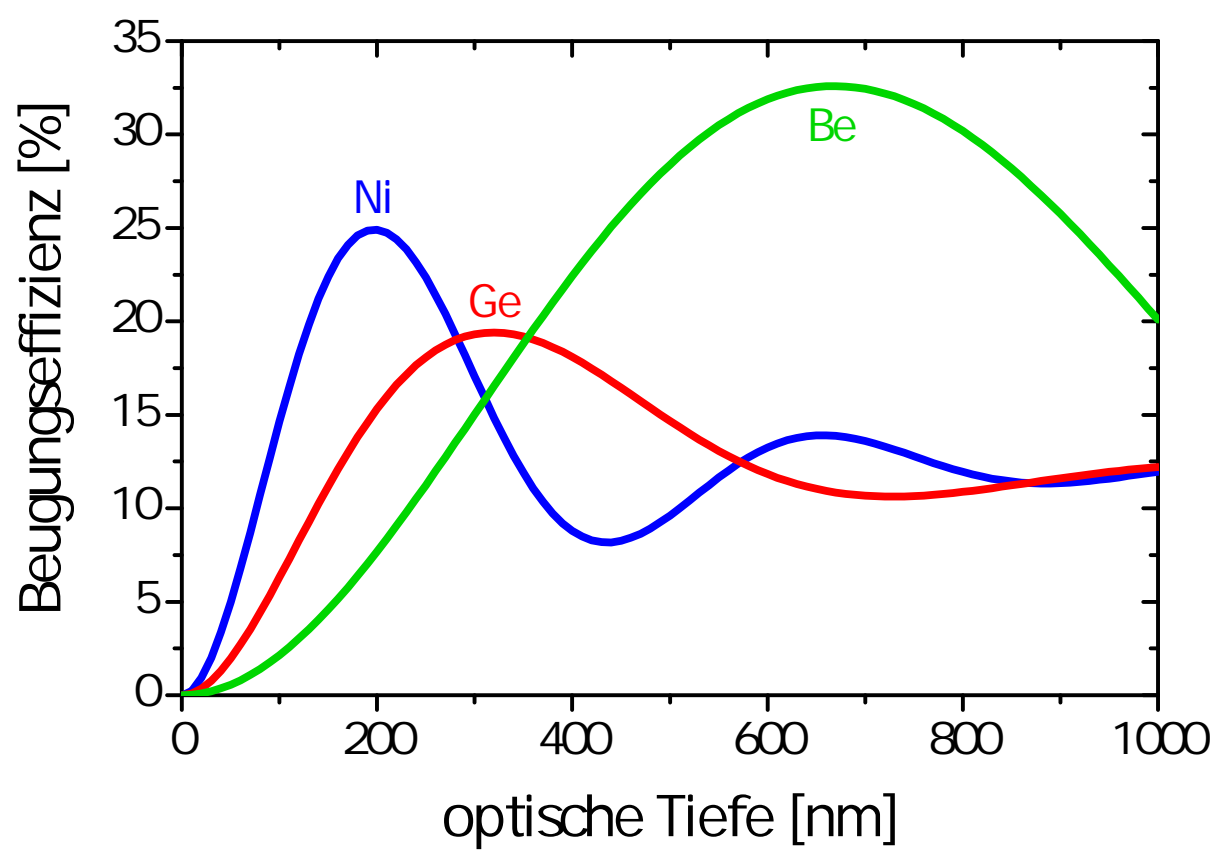

Abbildung 2.3: Berechnete Beugungswirkungsgrade kommerziell verwendeter Materialien für die Herstellung phasenschiebender ZP im weichen Röntgenwellenlängenbereich $(\lambda=2,88 \mathrm{~nm})$. Bei kleinen Werten von $k=\beta / \delta$ dominiert der Phasenschub gegenüber der Absorption, sodass sich z. B. für Beryllium eine hohe Effizienz von $33 \%$ nahe der theoretisch möglichen $40 \%$ ergibt. (Die $k$-Werte sind der CXRO-Datenbank entnommen [40]).

der Phasenschub um $2 \pi / L$ an, welcher durch unterschiedliche optische Tiefen der Unterzonen (genähertes Sägezahnprofil) korrigiert werden kann. Mit zunehmenden $L$ werden somit immer mehr Teilstrahlen in Phase addiert, sodass unter Vernachlässigung der Absorption die 0. sowie höhere Beugungsordnungen nach und nach komplett unterdrückt werden können. Die Effizienz der 1. Beugungsordnung

$$
\eta=\left(\frac{\sin (\pi / L)}{\pi / L}\right)^{2}
$$

steigt auf diese Weise sehr rapide an [42], wie es in Abbildung 2.4 graphisch veranschaulicht wird. Gleichzeitig sind die theoretisch möglichen Effizienzen aller hier beschriebenen Arten von ZP ( $L=1$ : Fresnel, $L=2$ : Rayleigh-Wood, $L>2$ : Multilevel) dargestellt. Dabei zeigt sich bereits bei einer quaternären ZP $(L=4)$ mit einer quasikontinuierlichen Phasenmodulation ein Effizienzanstieg auf mehr als 80\%.

Eine generelle Effizienzabschätzung für höhere Aspektverhältnisse, wie sie bei hochauflösenden ZP mit sehr kleinen äußeren Zonenbreiten, oder bei hohen optischen 


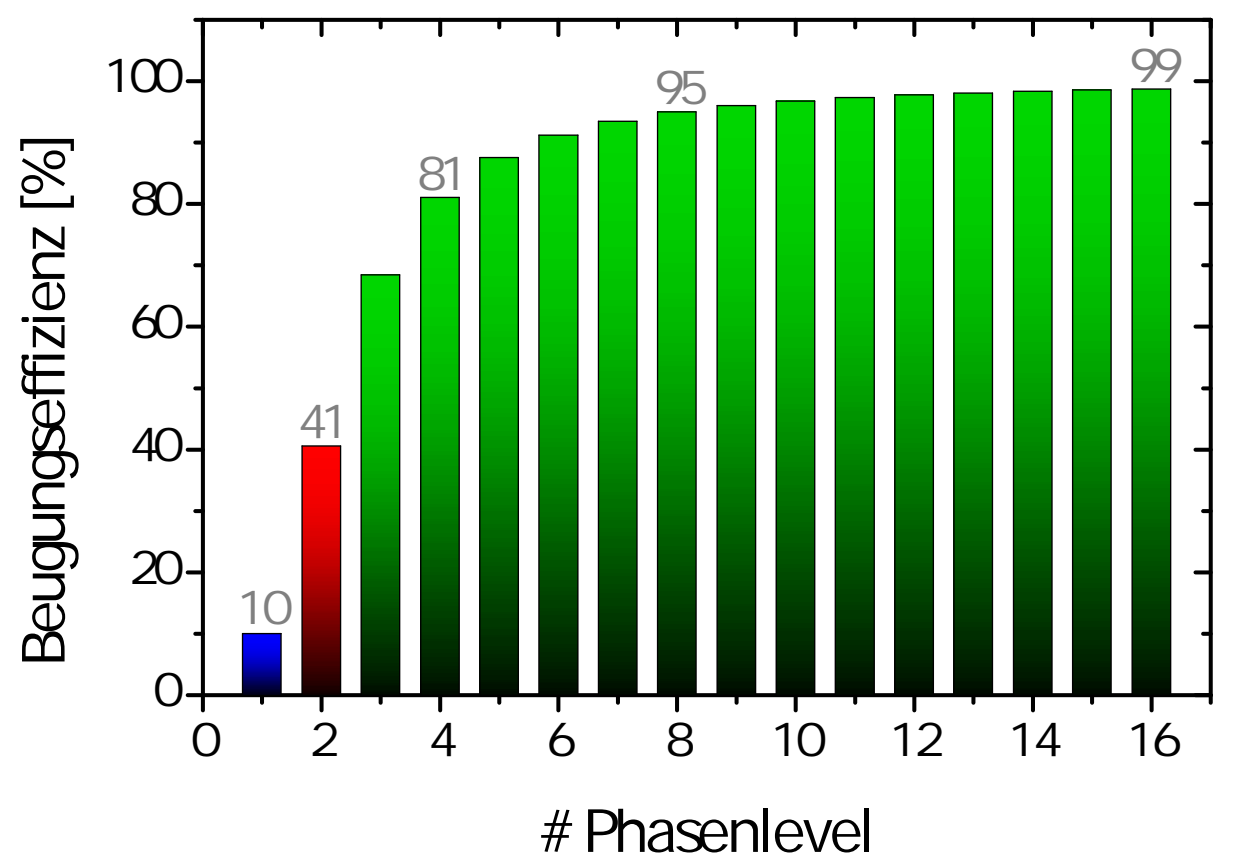

Abbildung 2.4: Theoretisch mögliche Effizienzen von Einzel- und Multilevel-ZP in Abhängigkeit der Anzahl der Phasenlevel, wobei $L=1$ einer reinen Amplituden-ZP (Fresnel) und $L=2$ einer binären Phasen-ZP (Rayleigh-Wood) entspricht. Mit zunehmendem Phasenlevel kann ohne Berücksichtigung der Absorption nahezu die komplette einfallende Strahlung in der 1. Beugungsordnung konzentriert werden (nach [42]).

Tiefen für eine ausreichende Wechselwirkung mit harter Röntgenstrahlung gegeben sind, stellt eine Beschreibung basierend auf der Theorie dynamisch gekoppelter Wellen dar, wonach die Effizienz nicht wie bei einer ideal dünnen ZP über die gesamte ZPStruktur konstant ist [43]. Durch Auftreten von Volumendiffraktionseffekten kann die Effizienz deshalb nicht mehr allein geometrisch behandelt werden. Betrachtet wird vielmehr die lokale Effizienz einer ZP als Funktion ihres Radius, die im Bereich nahe der optischen Achse durch die planare Beugungstheorie [39], für äußere Zonen jedoch durch die Erfüllung der Bragg-Gleichung dominiert wird. Mit zunehmender optischen Dicke steigt auch die Sensibilität der Bragg-Beziehung, sodass ein maximaler Wirkungsgrad bei hohen Aspektverhältnissen nur durch eine parabolische (planare Beleuchtung) oder elliptische Zonengeometrie (Punkt-zu-Punkt-Abbildung) erzielt werden kann [44]. SCHROER konnte anhand von Simulationen dynamisch gekoppelter Wellen zeigen, dass sich harte Röntgenstrahlung von $20 \mathrm{keV}$ mittels einer parabolischen Zonenform durch eine globale Reflexions-Erfüllung mit einer Effizienz von $63 \%$ fokussieren lassen [44]. Die einzelnen Zonen sind dabei so verkippt, dass sich der reflektierte Röntgenstrahl mit dem gebeugten in der 1 . Beugungsordnung konstruktiv überlagert. 


\subsubsection{Auflösungsvermögen}

Um die räumliche Aufösung einer ZP bestimmen zu können, soll zunächst deren Nomenklatur erläutert werden. Nach dem häufig benutzten Rayleigh-Kriterium können die von zwei Punktquellen stammenden Intensitätsverteilungen (Airy-Scheibchen) gerade noch unterschieden werden, wenn sich die abfallenden Flanken der Intensitäten 1. Ordnung vollständig überlagern [45]. Dieser minimale Abstand $R$ steht für die maximal mögliche Auflösung und ergibt sich anhand Formel $2.14 \mathrm{zu}$

$$
R=\frac{0,61 \lambda}{N A}=\frac{1,22 \lambda f}{D}
$$

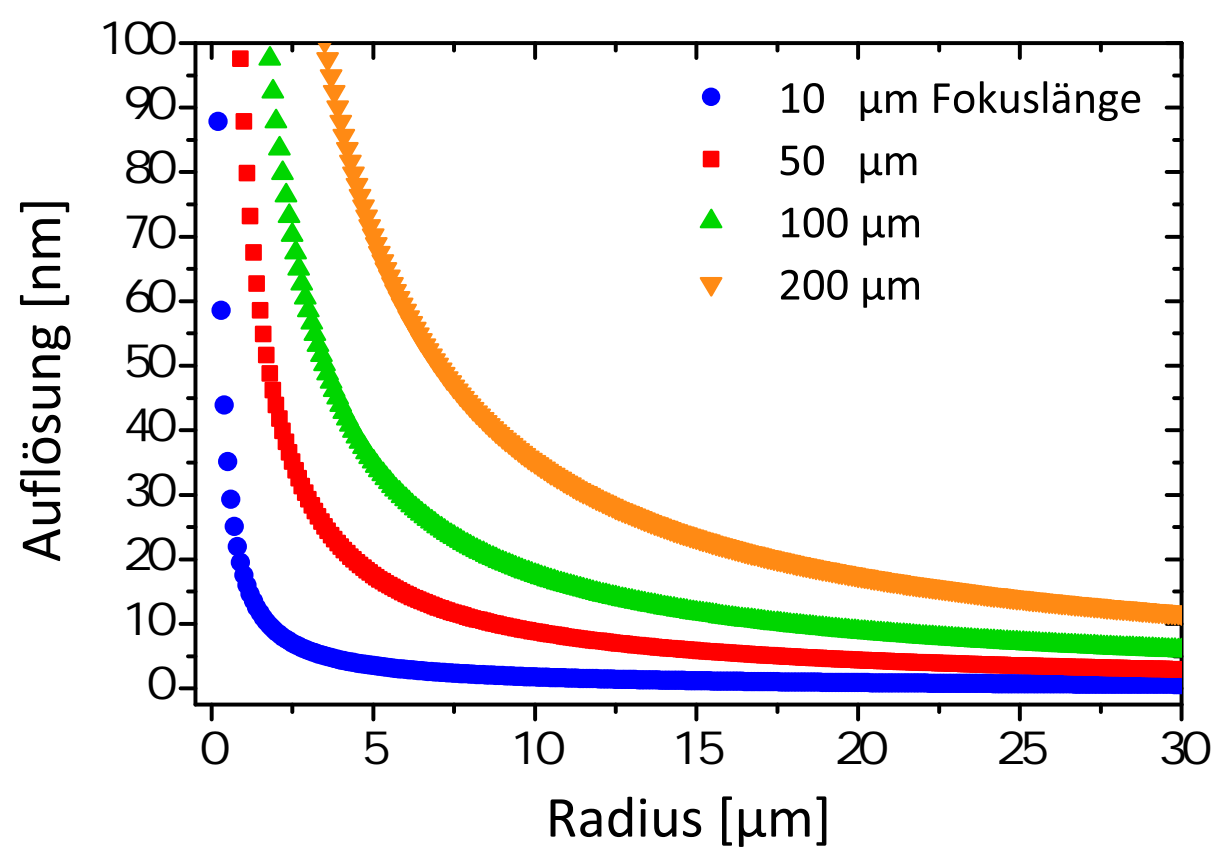

Abbildung 2.5: Veranschaulichung der theoretisch möglichen Auflösung einer vollständigen ZP bei fester Wellenlänge $(\lambda=2,88 \mathrm{~nm})$ und unterschiedlicher Fokuslänge, dargestellt in Abhängigkeit des Radius. Kleinere Fokuslängen führen bereits bei kleinerem ZP-Radius zu verbesserter Auflösung.

Die so erreichbare Auflösung wird bei fester Fokus- und Wellenlänge nur durch den Durchmesser der ZP bestimmt. In Abbildung 2.5 ist sie für verschiedene Fokuslängen bei einer festen Wellenlänge im weichen Röntgenbereich $(\lambda=2,88 \mathrm{~nm})$ dargestellt. Hohe Auflösungen lassen sich demnach bei verkleinerter Fokuslänge leichter realisieren, da die Anzahl an benötigten Zonen verringert wird. 
Nur für den Fall, dass alle Zonen zu einer kompletten ZP hergestellt werden (keine off-axis-ZP), kann die Auflösung anhand Formel 2.15 in Abhängigkeit der äußeren Zonenbreite formuliert werden.

$$
R \simeq 1,22 \Delta r
$$

Für höhere Beugungsordnungen gilt $R \sim 1 / m$, sodass sich bei vergrößerten numerischen Aperturen, bedauerlicherweise mit stark verringerten Effizienzen (s. Gl. 2.16) verbunden, nochmals höhere Auflösungen erzielen lassen.

Die Frage nach der maximal möglichen Auflösung, die mit einer Fresnelschen ZP zu realisieren ist, wird ebenfalls anhand der gekoppelten Wellentheorie diskutiert [44,46]. Im Gegensatz zu anderen Transmissionsoptiken wie z. B. Wellenleitern, bei denen die (theoretisch) erreichbare Auflösung knapp unterhalb der $10 \mathrm{~nm}$-Grenze vom kritischen Beugungswinkel $\theta_{c}=\sqrt{2 \delta}$ äußerer Totalreflexion abhängt [47], ist die Beugungslimitation einer ZP in erster Linie allein durch die äußere Zonenbreite bestimmt. Theoretisch wird anhand berechneter Wellenpropagation innerhalb einer verkippten ZPGeometrie mit hoher numerischer Apertur eine effiziente Fokussierung kleiner als $1 \mathrm{~nm}$ vorhergesagt, geltend jedoch nur bei einer globalen Erfüllung der Bragg-Bedingung [44]. Ist dies der Fall, kann die Aufösung sogar Werte unterhalb der äußersten Zonenbreite annehmen. Bei einer planaren Zonenstruktur würden Propagationseffekte innerhalb der ZP einen drastischen Abfall der Effizienz äußerer Zonen verursachen, welche somit nicht mehr effektiv zu einer Fokussierung beitragen würden.

\subsubsection{Entwicklung der Zonenplatten-Herstellung}

Obwohl es die technologischen Herstellungsmöglichkeiten von ZP derzeit noch nicht zulassen, existiert also kein physikalisches Limit, in der Röntgenmikroskopie eine räumliche Auflösung besser als $1 \mathrm{~nm}$ zu erreichen. Seit den grundlegenden Arbeiten von SCHMAHL et al., der schon 1981 eine experimentelle Auflösung von $10 \mathrm{~nm}$ vorhersagte, wurde in den letzten 30 Jahren enormer Forschungsaufwand betrieben, die Fabrikation von ZP voranzutreiben. Eine Übersicht der frühen Herstellungsmöglichkeiten wie z. B. die UV-Laserholographie, bei der durch Interferenz von sphärischer und planarer Wellenfronten ein ZP-Muster erzeugt wird, findet sich in [48-50]. Seit der Etablierung der fokussierten Ionenstrahltechnik wurden auch hiermit Versuche unternommen, ZPStrukturen zu fabrizieren [51-53]. 
Die konventionelle Herstellungsmethode hochauflösender ZP liegt jedoch in einem mehrstufigen Verfahren von Elektronenstrahl-Lithographie und reaktivem Ionenätzen [54] (s. Abb. 2.6). Das ZP-Muster wird dabei mit Hilfe eines Elektronenstrahls zunächst in einen elektronenempfindlichen Photolack geschrieben und durch Ionenätzen in eine dünne Metallschicht übertragen [55]. Die Positionsungenauigkeit des ZP-Musters sollte dabei nicht mehr als ein Drittel der äußeren Zonenbreite überschreiten [56]. Durch weiteres Ätzen wird die Zonenstruktur in eine Polymerschicht übertragen, die für elektrolytisches Abscheiden des Zonenmaterials, beispielsweise Nickel oder Gold, als Negativform benutzt und anschließend wieder entfernt wird $[57,58]$. Anstatt das eigentliche Zonenmaterial in einem Galvanik-Prozess aufzubringen, kann die ZPStruktur auch direkt in das vorgesehene Material (z. B. Germanium) durch zusätzliches reaktives Ionenätzen eingebracht werden [59].

(a)

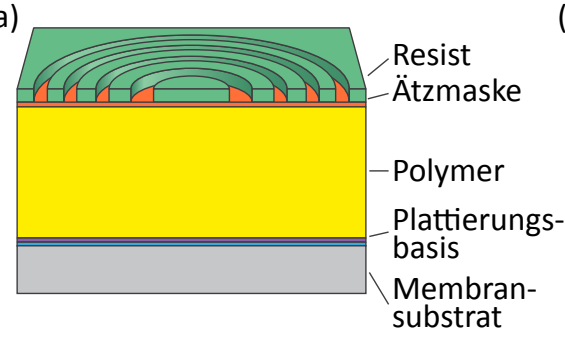

(b)

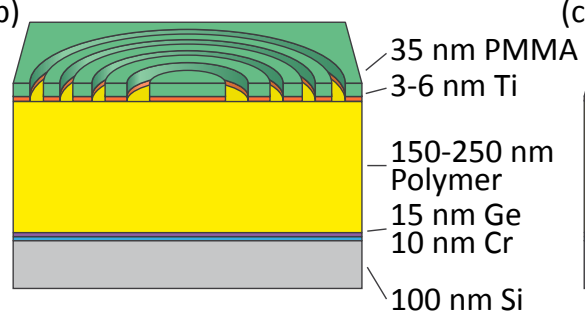

(c)

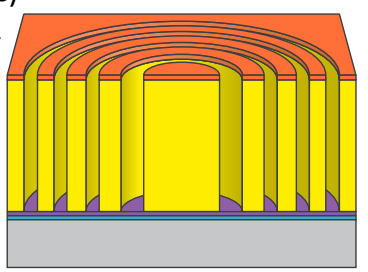

(d)

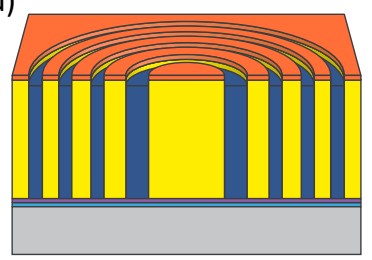

(e)

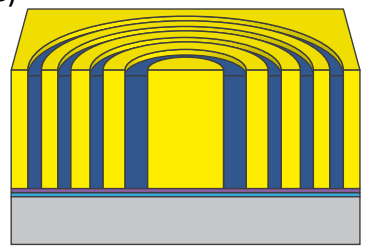

(f)

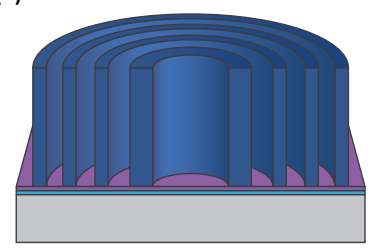

Abbildung 2.6: Prozessschritte der konventionellen ZP-Herstellung. Das mit Hilfe eines Elektronenstrahls in einen Photolack geschriebene ZP-Muster (a) wird durch Ätzen in eine dünne Metallschicht (b) und danach in eine Polymerschicht übertragen (c). Nach elektrolytischem Abscheiden des eigentlichen ZP-Materials (d) wird die Maske und das Polymer entfernt (e, f), um eine freistehende ZP zu erzeugen (nach [29]).

Unglücklicherweise birgt die Methode der Elektronenstrahl-Lithographie zwei wesentliche Herstellungsprobleme. Zum einen ist es enorm schwierig, kleinste äußere Zonenbreiten zu erzeugen, durch die die maximal mögliche Auflösung einer ZP bestimmt wird. Begrenzende Faktoren sind hier in der Größe des fokussierten Elektronenstrahls sowie in der Beschaffenheit des Entwicklers zu sehen, zumal die Entstehung von Sekundärelektronen innerhalb der Struktur die Herstellung hoher 
Zonendichten maßgeblich behindert [60]. Anderseits ist es bei der Röntgenmikroskopie (vor allem im harten Wellenlängenbereich) aufgrund der hohen Eindringtiefe bzw. der schwachen Wechselwirkung von Röntgenstrahlung von besonderer Bedeutung, hohe Aspektverhältnisse für eine optimale Effizienz-Ausschöpfung zu schaffen. Für einen Phasenschub von $\pi$ wird generell eine Zonentiefe größer als $100 \mathrm{~nm}$ gefordert, was gerade bei Mikro-ZP lithographisch eine absolute Herausforderung darstellt.

Durch eine Vielzahl an fortgeschrittenen Techniken lässt sich daher meist nur eines dieser beiden Defizite optimieren. Um die Beugungseffizienz von ZP zu erhöhen, wurde z. B. mit einer planaren, linienfokussierenden ZP-Struktur bei einer äußeren Zonenbreite von $100 \mathrm{~nm}$ eine Zonentiefe von $1,5 \mu \mathrm{m}$ erreicht, was ungefähr dem 10-fachen Wert einer konventionellen ZP entspricht [61]. Eine Drehung der Struktur erhöht die effektive Zonentiefe weiter, sodass eine 26\%-ige Effizienz bei einer Energie von $12,4 \mathrm{keV}$ (0,1 nm Wellenlänge) gemessen werden konnte. Eine Erhöhung des Aspektverhältnisses zweidimensionaler ZP kann auch durch permanentes Verbinden zweier exakt gleicher Einzel-ZP realisiert werden [62]. Ferner können ZP aus Nickel und Germanium kombiniert werden, indem die galvanisch hergestellte Nickel-Zonenstruktur als Maske für weiteres reaktives Ionenätzen in Germanium benutzt wird [63-65]. Auf diese Weise konnte bei einer äußeren Zonenbreite von nur $13 \mathrm{~nm}$ eine optische Tiefe von $80 \mathrm{~nm}$ erreicht werden. Höhere Aspektverhältnisse größer als 20:1 werden z. B. durch mehrfache Wiederholung sämtlicher konventioneller Lithographie- und Ätzprozesse erzielt [66]. Bei einer Zonentiefe von $900 \mathrm{~nm}$ konnten sogar partiell verkippte Strukturen zur lokalen Erfüllung der Bragg-Beziehung hergestellt werden [67]. Die höchste Effizienz wurde bisweilen von DI FABRIZIO mit einer quaternären Multilevel-ZP aus Nickel gemessen, die bei einer Energie von $7 \mathrm{keV}$ im Bereich harter Röntgenstrahlung 55\% beträgt [41].

Bezüglich einer Verbesserung der räumlichen Aufösung der Röntgenoptiken gelang es mitunter, zwei komplementäre ZP mit einer örtlichen Passgenauigkeit von $2 \mathrm{~nm}$ ineinander zu verschachteln [68], sodass sich aufgrund der geringeren lithographisch herzustellenden Zonendichte eine vollständige ZP mit einer äußeren Zonenbreite von $12 \mathrm{~nm}$ und einer Aufösung ebenfalls um die $12 \mathrm{~nm}$ fabrizieren ließ [24]. Ferner konnte unter Ausnutzung der 3. Beugungsordnung einer konventionellen ZP mit $\Delta r=25 \mathrm{~nm}$ eine Fokusgröße von $11 \mathrm{~nm}$ erzielt werden [25]. Eine weitere Möglichkeit, eine hohe Auflösung bei gleichzeitig adäquater Effizienz zu erlangen, stellt die Verwendung einer Verbund-ZP (engl. composite oder compound zone plate) dar, bei der eine zentrale ZP, bei der die 1. Beugungsordnung ausgenutzt wird, von einer äußeren ZP 
umgeben ist, deren 3. Beugungsordnung Röntgenstrahlung in der gleichen Fokusebene konzentrieren soll [69]. Somit konnte durch die Ausblendung der äußeren 1. Ordnung eine Auflösung von $25 \mathrm{~nm}$ erlangt werden [70]. Eine weitere vielversprechende Herstellungsmethode erweitert die übliche Elektronenstrahl-Lithographie mit nachträglicher Dünnschichtdeposition auf die Seitenwände geätzter Zonen, wodurch eine Verdoppelung der Zonendichte sowie ein hohes Aspektverhältnis ermöglicht wird [71]. Mit $15 \mathrm{~nm}$ äußerer Zonenbreite konnte bei einer Energie von $6,2 \mathrm{keV}(0,2 \mathrm{~nm})$ im harten Wellenlängenbereich erstmals eine Aufösung von $15 \mathrm{~nm}$ gemessen werden [72]. Bei weicher Röntgenwellenlänge von $1,03 \mathrm{~nm}(1,2 \mathrm{keV})$ wurde mit dieser Art von $\mathrm{ZP}$ mit $\Delta r=12,5 \mathrm{~nm}$ sogar eine Aufösung von $R=9 \mathrm{~nm}$ erreicht [26]. Damit konnte zum ersten Mal die $10 \mathrm{~nm}-$ Grenze mittels einer diffraktiven Transmissionsoptik durchbrochen werden.

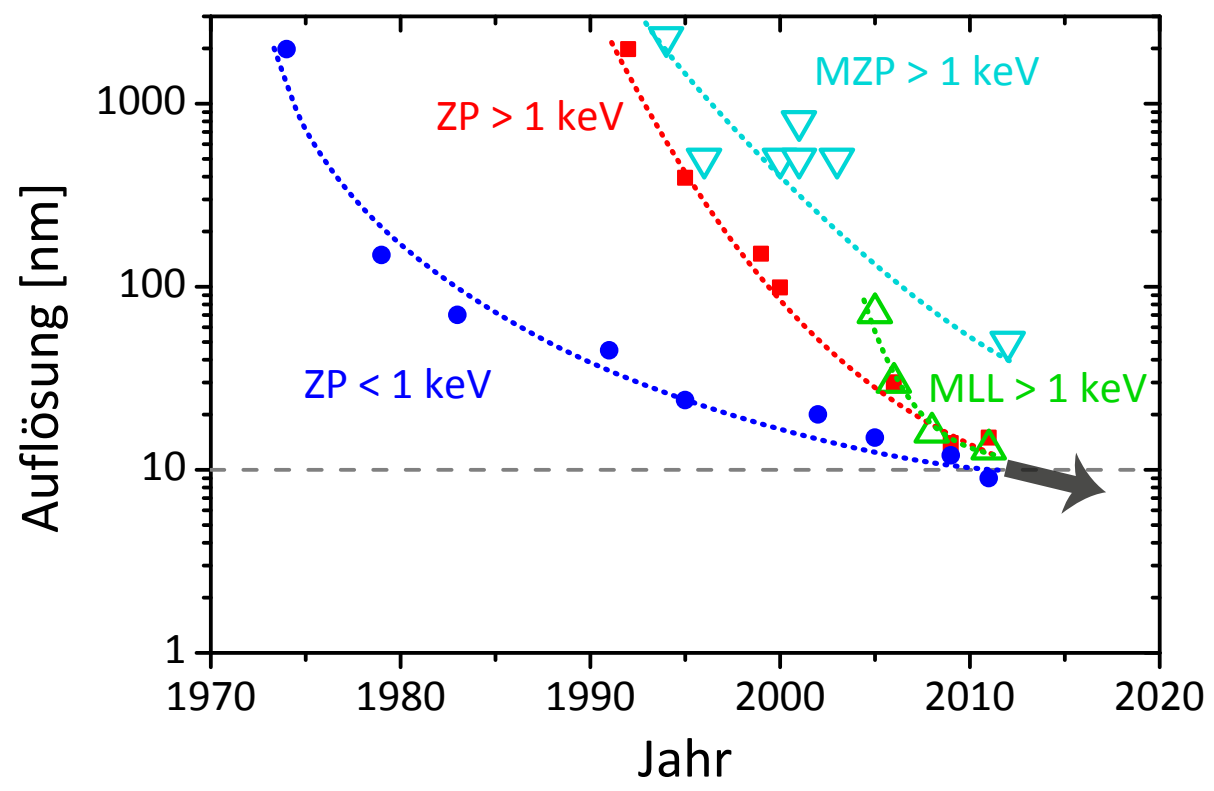

Abbildung 2.7: Zeitliche Entwicklung der bestmöglich erreichten Messdaten für die räumliche Auflösung von ZP, aufgegliedert nach weicher und harter Röntgenstrahlung. Mit immer besser werdenden Auflösungen nimmt auch die Herstellungsproblematik weiter zu. Diese kann durch die Verwendung von Multilagen-Laue-Linsen vereinfacht werden, welche eine rasante Entwicklung bzgl. minimaler Fokusgröße zeigen. (Die Daten sind aus [6] entnommen und durch weitere ergänzt $[24,26,31,72-82]$.)

In Abbildung 2.7 sind die besten Aufösungen von Fresnelschen ZP der vergangenen Jahre für weiche und harte Röntgenstrahlung zusammengefasst dargestellt. Da die frühen Anwendungsgebiete der Röntgenmikroskopie eher in der Untersuchung biologischer Zellen mit Dicken zwischen 0,1 und $10 \mu \mathrm{m}$ lagen, wurde die Entwicklung von ZP für den weichen Wellenlängenbereich, speziell im sog. „Wasserfenster“ $(2,3-4,4 \mathrm{~nm})$, 
bereits in den 70er Jahren vorangetrieben. Für modernere bildgebende Techniken wie Fluoreszenz-Mikroskopie oder Phasenkontrast-Tomographie, mit denen wesentlich dickere Proben analysiert werden können, erweist sich die Verwendung harter Röntgenstrahlung mit einer geringeren Absorption von Vorteil [5]. Die Herstellung entsprechender Optiken setzt jedoch hohe Aspektverhältnisse bei bestmöglicher Auflösung voraus, was die technologische Entwicklung von ZP im harten Röntgenbereich weitaus schwieriger gestaltet. Trotz stagnierender Fortschritte zeigen die Trends klar in Richtung einer sub-10 nm-Auflösung für zukünftige Anwendungen in der Röntgenmikroskopie. Bereits vorweg gegriffen sind außerdem die bisher erreichten Fokusgrößen von gesputterten Multilagen-Laue-Linsen und Multilagen-Zonenplatten aufgezeigt, welche im folgenden Kapitel 2.3 vorgestellt werden. Aufgrund der rasanten Entwicklung haben sich innerhalb der letzten Jahre vor allem linienfokussierende Multilagen-LaueLinsen als zukunftsweisende Alternative zu konventionellen Zonenplatten erwiesen.

\subsection{Multilagenbasierte Transmissionsoptiken}

Auf der Suche nach alternativen Herstellungsmethoden von ZP sind solche Optiken, die auf Multischichtsystemen basieren, sehr vielversprechend. Dabei werden die transparenten und absorbierenden Ringe einer ZP durch die Deposition von aperiodischen Multilagen aus zwei Materialien nachgebildet, die einen möglichst großen Unterschied im Absorptionskoeffizienten besitzen sollten. Auf planaren oder gekrümmten Substraten aufgebracht können somit ein- oder zweidimensionale ZP-Strukturen zur Erzeugung von Linien- oder Punktfoki hergestellt werden (s. Abb. 2.8).

Mit diesen beugenden Transmissionsoptiken, die als Multilagen-Laue-Linse (1DFokus, engl. Multilayer Laue Lens, MLL) bzw. Multilagen-Zonenplatte (2D-Fokus, engl. Multilayer Zone Plate, MZP) bezeichnet werden, gelingt es schlagartig, zwei wesentliche Probleme, die bei der lithographischen ZP-Manufaktur auftreten, zu überwinden: Sowohl kleinste äußere Zonenbreiten, die die Auflösung einer ZP bestimmen, als auch nahezu beliebig große Aspektverhältnisse für eine optimale Effizienzausbeute sind simultan realisierbar.

Die Einzelschichtdicken der MLL oder MZP leiten sich wiederum nach dem Zonenplattenbildungsgesetz ab, welches den Radius jeder Zone bestimmt. Müssen die dickeren Zonen z. B. bei der Beschichtung eines Drahtes, welcher den Platz einiger 

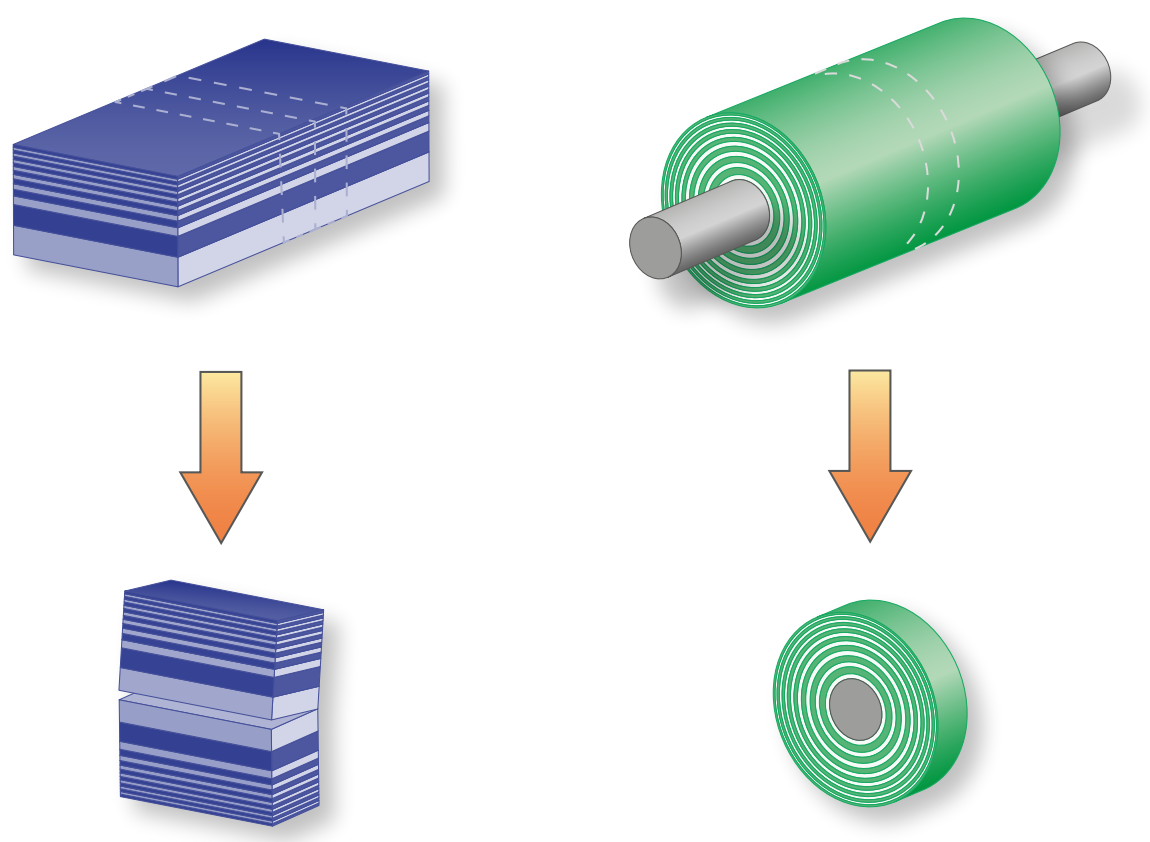

Abbildung 2.8: Schematische Darstellung von Multilagen-Laue-Linsen und Multilagen-Zonenplatten. Mit der Herstellung solcher alternativer Transmissionsoptiken sind kleinste äußere Zonenbreiten bei nahezu beliebigen optischen Tiefen möglich.

Zonen einnimmt oder aufgrund einer langen Herstellungsdauer ausgelassen werden, wird Gleichung 2.9 mit dem off-axis-Radius $r_{0}$ in folgender Form abgewandelt [83]:

$$
r_{n}^{2}=r_{0}^{2}+n \lambda f .
$$

Je nachdem, wie viele Zonen ausgelassen werden, ändert sich aufgrund einer geringeren Aperturgröße die Effizienz sowie die Aufösung einer multilagenbasierten Transmissionsoptik, welche im Folgenden theoretisch betrachtet werden sollen. Darüber hinaus können fehlerhafte Schichtdicken sowie die Existenz von Grenzflächenrauigkeiten die Effizienz und die Aufösung erheblich beeinträchtigen.

\subsubsection{Beugungseffizienz}

Da bei einer MLL und MZP nahezu jedes Aspektverhältnis realisierbar ist, lassen sich auf relativ einfache Weise effiziente phasenschiebende ZP-Strukturen herstellen. Die benötigte optische Tiefe für einen Phasenschub um $\pi$ ergibt sich im einfachsten Fall bei einem 2-Materialsystem unter Vernachlässigung der Absorption zu 


$$
\Delta t=\frac{\lambda}{2\left(\delta_{2}-\delta_{1}\right)}
$$

Eine theoretische Effizienzabschätzung, welche die Phase und die Absorption der einzelnen Zonenmaterialien berücksichtigt, wurde von YUN et al. vorgenommen. Dazu wird der in Gleichung 2.18 angegebenen Berechnung für eine konventionelle ZP (Zonenmaterial und Vakuum abwechselnd) in Abhängigkeit der optischen Tiefe $\Delta t$ weitere Terme hinzugefügt [84]:

$$
\eta=\frac{1}{(\pi m)^{2}}\left(\gamma_{1}^{2}+\gamma_{2}^{2}-2 \gamma_{1} \gamma_{2} \cos \left[2 \pi \Delta t\left(\delta_{2}-\delta_{1}\right) / \lambda\right]\right)
$$

Die Absorption der beiden Materialien ist dabei in $\gamma_{i}=\exp \left(-2 \pi \Delta t \beta_{i} / \lambda\right)$ mit $i=1,2$ enthalten.

Neben der Herstellung einer binären phasenschiebenden MLL/MZP lässt sich die Effizienzausbeute auf zwei weitere Arten erhöhen: 1. durch zusätzliche Ausnutzung der Reflexion von Röntgenstrahlung an den Grenzflächen [85,86] und 2. durch sukzessive Phasenmodulation anhand gradueller Brechungsindizes [87,88]. Um im ersten Fall die Effizienz einer MLL/MZP durch eine Kombination von Diffraktion und Reflexion zu erhöhen, wird die lokale oder sogar globale Erfüllung der Bragg-Bedingung an den Grenzflächen der einzelnen Zonen angestrebt. Bei größeren Aspektverhältnissen ist die Ausnutzung dieses Effekts sogar essentiell, da die lokale Effizienz bei optischen Tiefen $\Delta t>(2 \Delta r)^{2} / \lambda$ in Abhängigkeit des ZP-Radius rapide abnimmt [85, 89]. Bei einer optimalen optischen Tiefe ist dies für äußere Zonenbreiten $\Delta r<10 \mathrm{~nm}$ der Fall. In Abbildung 2.9 sind verschiedene MLL/MZP-Geometrien skizziert, welche von links nach rechts betrachtet immer besser für eine hohe Effizienzausbeute optimiert sind.

Den einfachsten Fall stellt die flache, konventionelle ZP-Struktur dar. Bei großen optischen Tiefen treten hier jedoch effizienzmindernde Volumendiffraktionseffekte in den Vordergrund, sodass hohe Effizienzen nur durch Superposition von Diffraktion und Reflexion möglich sind. Die vorausgesetzte Einhaltung der Bragg-Beziehung ist hier nur für Zonen nahe der optischen Achse gewährleistet, sodass die lokale Effizienz mit steigendem Radius der ZP bis auf Null abnimmt. Bei einer gekippten Geometrie zweier MLL-Hälften wird der Verkippungswinkel so gewählt, dass die Bragg-Bedingung in einem schmalen äußeren Zonenbereich erfüllt ist und Reflexion in eine Beugungsordnung stattfindet. In einer keilförmigen Anordnung der MLL/MZP (engl. wedged) 


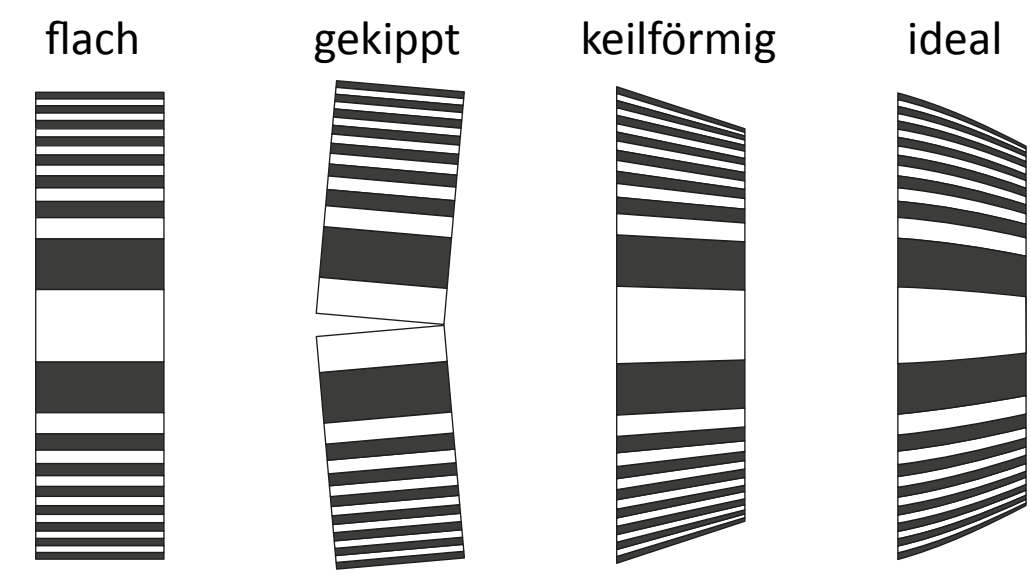

Abbildung 2.9: Mögliche Anordnungen von MLL/MZP zur gesteigerten Effizienzerhöhung. Von links nach rechts betrachtet erfüllen immer größere Zonenbereiche die Bragg-Bedingung, sodass die integrale Effizienz durch konstruktive Überlagerung von Diffraktion und Reflexion erhöht werden kann (nach [85]).

ist jede Zone mit einem individuellen linearen Dickengradienten versehen, um eine globale Reflexion der transmittierenden Röntgenstrahlung zu erhalten. Beschrieben wird diese Dickenabnahme durch ein modifiziertes Zonenplattenbildungsgesetz nach YAN et al. [85]:

$$
r_{n}=a_{n}(z) \sqrt{n \lambda f+\frac{n^{2} \lambda^{2}}{4}}, \quad a_{n}=1-\frac{z}{2 f(1+n \lambda / 4 f)}
$$

An der Eintrittsseite der MLL/MZP $(z=0)$ wird das Zonenplattenbildungsgesetz zur konventionellen Form reduziert. Ein großer Vorteil dieser Geometrie ist, dass bis auf die 1. Beugungsordnung alle anderen (einschließlich des Nullstrahls) signifikant unterdrückt werden. In der Mikroskopie bräuchte somit keine ordnungsselektierende Blende benutzt zu werden. Lokal betrachtet verläuft die Effizienz allerdings nicht konstant über den gesamten Radius, da es in einigen Bereichen zu einer Abweichung der Phase kommt. Dieser Effekt wird durch die ideale kurvenförmige Geometrie behoben: Hierbei muss das Zonenprofil zur Erfüllung der Bragg-Beziehung bei gleichbleibender konstruktiver Phasenaddition parabolisch (planare Beleuchtung) oder elliptisch (Punkt-zu-PunktAbbildung) verlaufen [90]. Auf diese Weise konnte bei einer Photonenenergie von $19,5 \mathrm{keV}$ eine hohe integrale Effizienzausbeute einer $\mathrm{WSi}_{2} / \mathrm{Si}-\mathrm{MLL}$ von $67 \%$ simuliert werden [85].

Ähnlich der in Kapitel 2.2.1 vorgestellten Multilevel-ZP können auch bei MLL und MZP quasi-kontinuierliche Phasenmodulationen realisiert werden, um eine Effizienz- 
erhöhung zu erzielen. Bei diesen sog. Quasi-Kinoform-ZP wird der Phasenübergang nicht anhand unterschiedlicher optischer Tiefen innerhalb einer Zone erreicht, sondern durch eine graduelle Änderung im Brechungsindex der verwendeten Materialien [87]. Bei gleichbleibender optischer Tiefe wird somit ein Sägezahnprofil des Brechungsindex durch eine kompositionelle Variation der schwach und stark absorbierenden Materialien angestrebt. Die Effizienz einer solchen ZP mit $L$ Multilevel ergibt sich unter Berücksichtigung der Absorption zu

$$
\eta=\left[\frac{1}{2 \pi}\left(\sum_{l=1}^{L} T_{l} \int_{\frac{2 l \pi}{L}-\frac{\pi}{L-1}}^{\frac{2 l \pi}{L}} e^{\mathrm{i} \theta} \mathrm{d} \theta\right)\right]^{2},
$$

wobei der Transmissionskoeffizient $T_{l}=\exp \left(-2 \pi \beta_{l} \Delta t / \lambda\right)$ mit $l=1,2, \ldots, L$ die Abschwächung jeder Unterzone beschreibt [88]. Für die Realisierung einer MultilevelStruktur muss allerdings auf eine niedrige Interdiffusion der verwendeten Materialien geachtet werden, da bei einer Diffusionslänge $\Delta x>2 / 3 \Delta r$ mit einem Intensitätsabfall der transmittierten Röntgenstrahlung gerechnet werden muss [91].

Bei multilagenbasierten Optiken mit kleinen äußeren Zonenbreiten kann ein Effizienzverlust außerdem durch auftretende Grenzflächenrauigkeiten verursacht werden, welcher durch das Verhältnis von Rauigkeit und Zonenbreite $\xi=\sigma / \Delta r$ beschrieben wird [92]. Nimmt die Rauigkeit weniger als $10 \%$ der Schichtdicke ein $(\xi=0,1)$, ergibt sich eine vernachlässigbare lokale Verringerung der Effizienz unterhalb $5 \%$. Für $\xi=0,5$ lässt sich in der Simulation von YAN allerdings schon ein merklicher Abfall der Effizienz um 50\% feststellen [92]. Der Grund hierfür liegt in einer starken Ausschmierung der Elektronendichte von absorbierender und transmittierender Zone, sodass die Beugungsintensität deutlich abgeschwächt wird. Ist dies der Fall, tragen jene Zonen auch nicht mehr zu einer effektiven Fokussierung der Röntgenstrahlung bei.

\subsubsection{Auflösungsvermögen}

Im Grunde entspricht die maximal erreichbare, beugungslimitierte Auflösung einer multilagenbasierten Zonenplattenoptik der einer konventionell hergestellten ZP. Häufig werden aufgrund zu langer Herstellungsdauer die dicksten Schichten nahe der optischen Achse ausgelassen, sodass sich die Auflösung in diesem off-axis-Fall mit einer gesamten Breite aller deponierten Zonen $b<D / 2$ (vgl. Formel 2.21) zu 


$$
R=0,61 \frac{\lambda f}{b}
$$

reduziert. Wird zudem nur eine Hälfte einer MLL hergestellt, wie es in der Literatur oft der Fall ist, verschlechtert sich die Aufösung um den Faktor 2. Soll die minimale Fokusgröße näherungsweise durch die äußere Zonenbreite ausgedrückt werden, ergibt sie sich nach LIU und KANG et al. zu $R=1,22 \Delta r / p$ oder $R=\Delta r / p$ (zirkularer oder linearer Fokus) mit dem Bruchteil $p$ einer vollständig hergestellten Zonenplattenoptik [89,93]. Simulationen von MASER et al. haben diesbezüglich gezeigt, dass bis zu 25\% einer vollständigen ZP ohne eine sichtbare Verschlechterung der Auflösung ausgelassen werden können [30].

Um mit Hilfe von eindimensionalen MLL einen zweidimensionalen Punktfokus zu erzeugen, lassen sich zwei um $90^{\circ}$ versetzte MLL verwenden, wobei die zweite Linse eine verkürzte Brennweite besitzen muss, um Röntgenstrahlung durch beide Teillinsen in einem Punkt konzentrieren zu können [30]. Dabei kann eine ungenügende Justage der beiden Teillinsen bzgl. Bragg-Winkel und Abstand zueinander schnell zu einer Fokusverbreiterung führen [94].

Im harten Röntgenwellenlängenbereich bei optischen Tiefen größer als $(2 \Delta r)^{2} / \lambda$ kann eine maximale Auflösung (1D oder 2D) nur durch modifizierte ZP-Geometrien, wie es in Kapitel 2.3.1 vorgestellt wurde, erreicht werden. Da die lokale Effizienz bei unverkippten Zonen kleiner als $10 \mathrm{~nm}$ durch Auftreten von Volumendiffraktionseffekten bis auf weniger als $1 \%$ abnimmt, verringert sich die effektive numerische Apertur gegenüber der tatsächlichen derart, dass die äußeren Zonen nicht zu einer Fokussierung beitragen [90]. Der Grund hierfür liegt in dem unterschiedlichen Diffraktionsverhalten einer MLL/MZP mit hohem Aspektverhältnis. So verhält sich die Optik nahe der optischen Achse, wo die Zonenbreite relativ groß ist, wie ein dünnes Gitter (Beugung am Spalt) und im äußeren Zonenbereich mit kleinen $\Delta r$ wie ein dynamisch beugender Kristall (Laue-Diffraktion) [30,89]. Letzterer Effekt führte schließlich zu der Namensgebung der Multilagen-Laue-Linse (MLL) durch MASER et al. [30].

Erst durch eine individuelle lineare Krümmung jeder Zone zur Einhaltung der BraggBeziehung („wedged“-Struktur, s. Abb. 2.9) konnte anhand berechneter Strahlpropagation dynamisch gekoppelter Wellen bei einer äußeren Zonenbreite von $\Delta r=0,25 \mathrm{~nm}$ und eingestrahlter Photonenenergie von 19,5 keV eine hohe Auflösung von $R=0,34 \mathrm{~nm}$ demonstriert werden [85]. Mittels einer parabolischen Zonenform, welche neben der Einhaltung der Bragg-Bedingung auch eine richtige Phasenaddition berücksichtigt, 
wurde auf diese Weise eine theoretische Auflösung von $R=0,21 \mathrm{~nm}$ ermittelt, welche sogar kleiner als die angenommene äußere Zonenbreite ist.

Theoretisch existiert somit keine physikalische Grenze, mittels MLL und MZP Auflösungen kleiner als $1 \mathrm{~nm}$ zu erreichen. Praktisch stellt jedoch bei der Dünnschichtdeposition im atomaren Bereich die Rauigkeit den limitierenden Faktor dar, welche bei sub-nm-Schichtdicken nicht zu vernachlässigen ist. Nimmt das Verhältnis $\xi=\sigma / \Delta r$ zwischen Rauigkeit und Zonenbreite einen Wert größer als 0,5 an, ist in theoretischer Betrachtung eine beachtliche Schwächung der Fokussierung (z.B. auf das Dreifache bei $\xi=0,8)$ festzustellen [92]. Darüber hinaus ist es bei einer Multischicht-Optik ein möglicher Schichtdickenfehler, der eine systematische Abweichung der Zonenpositionierung vom Zonenplattenbildungsgesetz und somit eine Verringerung der erreichbaren Auflösung hervorruft [90]. Während hier die Effizienzausbeute weitestgehend unberührt bleibt, führt diese Unregelmäßigkeit zu einem stark gestörten Fokusprofil. Durch auftretende Phaseneffekte treten auf einer Seite des Hauptmaximums mehrere aufösungsmindernde Seitenmaxima auf, welche anhand von Simulationen und Fokussierungsexperimenten gezeigt werden konnten [95].

Sämtliche theoretische Betrachtungen der möglichen Auflösung von MLL und MZP wurden in der Literatur bislang nur für harte Röntgenstrahlung vollzogen. Bei weicher Röntgenstrahlung wäre mit Hilfe multilagenbasierter Optiken sogar eine wellenlängenbegrenzte Auflösung möglich.

\subsubsection{Entwicklung der MLL- und MZP-Herstellung}

Bereits 1966 wurde die Herstellung von ZP mittels Vakuumdeposition von dünnen Filmen von HART et al. vorgeschlagen [96]. Erst Anfang der 80er Jahre wurde diese Methode von RUdolPH et al. für kurze Wellenlängen im Röntgenbereich adaptiert, indem alternierende Lagen aus absorbierenden (oder phasenschiebenden) und möglichst transparenten Materialien auf einen rotierenden Draht gesputtert wurden [48,97]. Der Draht, welcher während der Deposition als Substrat und später als innerer beam stop dient, wurde anschließend in dünne Scheiben geschnitten und auf eine adäquate Dicke ausgedünnt bzw. poliert, um eine effiziente ZP zu erhalten. Diese Technik wurde primär für harte Röntgenstrahlung entwickelt, da dort die Realisierung großer Aspektverhältnisse weitaus einfacher ist als es mit herkömmlicher ElektronenstrahlLithographie möglich wäre [84]. Die ersten bildgebenden Experimente mit harter Röntgenstrahlung und einer Auflösung von ca. $3 \mu \mathrm{m}$ bei einer Effizienz von 4,5\% wurden 
mit einer MZP, bestehend aus $20 \mathrm{WSi}_{2} / \mathrm{C}-\mathrm{Multischichten,} \mathrm{von} \mathrm{SAITOH} \mathrm{et} \mathrm{al.} \mathrm{erreicht,}$ während die erste phasenschiebende ZP mit einer Effizienz von $19 \%$ von BIONTA et al. hergestellt wurde $[83,98]$.

Aufgrund der starken Ausbildung kumulativer Rauigkeiten konnte bis 2009 lediglich die Anwendbarkeit von MZP mit maximal 70 Einzelschichten und minimalen äußeren Zonenbreiten von $160 \mathrm{~nm}$ bei immer höheren Photonenenergien bis zu $200 \mathrm{keV}$ mit erreichten Auflösungen zwischen 5 und $0,5 \mu \mathrm{m}$ demonstriert werden [77,79,81,99-101]. Mittels der Deposition von 134 Lagen $\mathrm{MoSi}_{2} / \mathrm{Si}$ mit Schichtdicken zwischen 50 und $40 \mathrm{~nm}$ auf einem Glasdraht gelang es schließlich, eine Auflösung von $50 \mathrm{~nm}$ zu erreichen [82]. Mit Hilfe einer keilförmigen ZP-Struktur wurde bei Auflösungen von $280 \mathrm{~nm}$ bzw. $50 \mathrm{~nm}$ (volle und partielle Beleuchtung) aufgrund der globalen Erfüllung der Bragg-Beziehung eine Effizienz von 52\% erzielt. [102]. Durch die erstmals realisierte keilförmige Anordnung wurde hierbei sogar die Ausblendung der - 1. Beugungsordnung erfolgreich demonstriert, sodass ohne eine ordnungsselektierende Blende gearbeitet werden konnte. Eine Abschwächung der -1. Beugungsordnung mit einhergehender Intensitätsverstärkung der +1 . Beugungsordnung wurde auch bei der Untersuchung von $\mathrm{Al} / \mathrm{Cu}-\mathrm{Multilevel-MZP}$ gefunden [87]. Mittels der Herstellung von $4-6$ MultilevelMZP konnten bei Auflösungen um $1 \mu \mathrm{m}$ ebenfalls Effizienzen von $50-52 \%$ erreicht werden [103-105].

Zu Beginn der im Rahmen dieser Arbeit hergestellten Optiken waren ausschließlich Veröffentlichungen zu gesputterten MZP bei harter Röntgenstrahlung vorhanden. Seit kurzem wurde die zweidimensionale Fokussierung im weichen Röntgenbereich mittels Atomlagenabscheidung (engl. atomic layer deposition, ALD) mit einer Auflösung von $39 \mathrm{~nm}$ gezeigt [106]. Die für ALD bevorzugte Materialwahl $\mathrm{Al}_{2} \mathrm{O}_{3} / \mathrm{Ta}_{2} \mathrm{O}_{5}$ führte allerdings nur zu einer Effizienz von 3,7\%. Ähnlich wie auch in dieser Arbeit wurde die Fabrikation der MZP mittels FIB durchgeführt. Auch die plasmaunterstützte chemische Gasphasenabscheidung (engl. surface-plasma chemical vapor deposition, SPCVD) wurde als alternative Herstellungsmethode von MZP getestet [107, 108]. Aufgrund der hohen Depositionsrate konnten bisher keine Schichtdicken unterhalb $100 \mathrm{~nm}$ realisiert werden, sodass diese Depositionsmethode eher für die Herstellung von Kondensor-MZP geeignet ist.

Die langwierige Entwicklung der MZP-Herstellung ist hauptsächlich der Ausbildung von kumulativen Rauigkeiten innerhalb der Schichtsysteme geschuldet. Eine Vielzahl an Veröffentlichungen über kumulative Rauigkeiten zeigt, dass selbst bei planar deponierten Multischichten exakte Herstellungsparameter gefunden werden müssen, um eine 
additive Rauigkeitsentwicklung unterdrücken zu können [109-113]. Bei einer Schichtherstellung auf gekrümmten Substraten kommt erschwerend die Deposition unter flachen Einfallswinkeln hinzu, sodass durch einen auftretenden Abschattungseffekt die Entstehung kumulativer Rauigkeiten bei der Sputterdeposition zusätzlich begünstigt wird [114]. Dieser Effekt konnte durch die Ausblendung flacher Depositionswinkel mittels eines dünnen Schlitzes zwischen Target und Substrat geringfügig eingeschränkt werden [115].

Neben den Herstellungsparametern spielt für die Deposition hochwertiger Multischichten die Materialauswahl eine wesentliche Rolle, deren Qualität durch die Grenzflächenstruktur beeinflusst wird. Auf der Suche nach minimaler kumulativer Rauigkeit wurden verschiedenste Materialsysteme wie z. B. $\mathrm{Ag} / \mathrm{C}, \mathrm{W} / \mathrm{C}, \mathrm{Cr} / \mathrm{C}, \mathrm{Cu} / \mathrm{C}$, $\mathrm{Cu} / \mathrm{Al}, \mathrm{WSi}_{2} / \mathrm{C}, \mathrm{Ni} / \mathrm{SiO}_{2}, \mathrm{NiCr} / \mathrm{SiO}_{2}, \mathrm{MoSi}_{2} / \mathrm{Si}$ und $\mathrm{Al}_{2} \mathrm{O}_{3} / \mathrm{Ta}_{2} \mathrm{O}_{5}$ für die $\mathrm{MZP}-$ Herstellung untersucht $[50,78,80,83,102,106,116,117]$, wobei mit letzteren beiden die besten Strahlfokussierungen erzielt wurden. Dabei kommt es nicht nur auf optimale Wachstumseigenschaften, sondern auch auf eine Langzeitstabilität der Depositionsraten der verwendeten Materialien an, um eine fehlerhafte Zonenpositionierung nach dem Zonenplattenbildungsgesetz zu vermeiden. Darüber hinaus haben DüvEL et al. bei gesputterten MZP eine Abhängigkeit der Rauigkeit von dem Drahtdurchmesser festgestellt, sodass Drahtbeschichtungen unterhalb $50 \mu \mathrm{m}$ Durchmesser als nicht sinnvoll erschienen [117]. Auch die Oberflächenbeschaffenheit sowie die Abweichung des Drahtes von der Zylinderform führt zu einer Verringerung der erreichbaren Auflösung, weshalb die Verwendung von Glasdrähten zweckmäßiger als die wegen hoher Absorption häufig benutzten $\mathrm{Au}$-Drähte erscheint $[102,106]$.

Aufgrund der lang anhaltenden MZP-Herstellungsprobleme wurde sich gleichzeitig auf die Multischichtdeposition auf planaren Substraten konzentriert, um in erster Linie multilagenbasierte Bragg-Fresnel-Linsen herzustellen [118-120]. Die erste MLL in Laue-Geometrie wurde von MASER et al. mit 470 alternierenden $\mathrm{WSi}_{2} / \mathrm{Si}_{-}$-Schichten und einer äußeren Zonenbreite von $60 \mathrm{~nm}$ realisiert, welche einen Linienfokus von $72 \mathrm{~nm}$ erzeugte [31]. Ein wesentlicher Vorteil gegenüber der MZP-Herstellung ist die Verringerung von Positionierungsfehlern der kleinsten aufösungsbestimmenden Zonen, da diese gleich zu Beginn der Deposition auf einem planaren Substrat hergestellt werden können [30]. Die gesputterten Multischichten werden in einem aufwändigen Verfahren bestehend aus Sägen, mechanischem Polieren, Ionenpolieren und Plasmareinigen auf eine entsprechende optische Tiefe ausgedünnt [121]. Eventuell auftretende Sägeschäden können dabei zu einer gestörten Schichtstruktur und somit zu einer massiven Fokus- 
verbreiterung führen. Ebenfalls kann eine unzureichende Haftung zwischen Schicht und Substrat sowie hohe innere Spannungen zu einem Abreißen der Multischicht während des Sägens führen [31]. Zur Vermeidung solcher Fabrikationsschäden können MLL alternativ durch reaktives Ionenätzen fabriziert werden, wobei hier die Erzeugung einer anisotropen Querschnittsfläche für eine konstante Fokuslänge problematisch ist [122]. Vor dem Ausdünnen können zur Herstellung einer vollen MLL-Struktur zwei MLLHälften mit einer niedrig schmelzenden AuSn-Zwischenschicht verklebt werden [123].

Die Anforderungen an die Materialsysteme sind neben ihrer optischen Eigenschaften eine niedrige intrinsische Spannung, eine adäquate Haftung der Multischicht zum Substrat sowie niedrige intrinsische Rauigkeiten [31,113]. Zur Multischichtherstellung für die MLL-Fabrikation wurden bisher $\mathrm{W} / \mathrm{Si}, \mathrm{WSi}_{2} / \mathrm{Si}, \mathrm{Mo} / \mathrm{Si}$ und $\mathrm{MoSi}_{2} / \mathrm{Si}$ verwen$\operatorname{det}[31,86,124]$, wobei die Silizid-Systeme aufgrund besserer Wachstumseigenschaften bei der Sputterdeposition bisher beste Aufösungen von $16 \mathrm{~nm}\left(\mathrm{WSi}_{2} / \mathrm{Si}\right)$ und $13 \mathrm{~nm}$ $\left(\mathrm{MoSi}_{2} / \mathrm{Si}\right)$ mit jeweiliger äußerer Zonenbreite von $\Delta r=5 \mathrm{~nm}$ zeigten $[75,125]$. Zweidimensionale Foki-Erzeugung durch die Kreuzung von senkrechter und waagrechter Einzellinsen konnten bisher mit Auflösungen von ca. $100 \times 100 \mathrm{~nm}^{2}\left(\mathrm{MoSi}_{2} / \mathrm{Si}\right)$ und $25 \times 27 \mathrm{~nm}^{2}\left(\mathrm{WSi}_{2} / \mathrm{Si}\right)$ demonstriert werden [75,126]. Da bei diesen zweidimensionalen Geometrien die beiden auf verschiedenen Halterungen befindlichen Einzellinsen sehr genau justiert werden müssen, sind hochstabile und vibrationsarme mechanische Positionierungssysteme vonnöten $[127,128]$.

Um die Auflösung gesputterter Transmissionsoptiken in Zukunft zu optimieren, sollen MLL mit hoher numerischer Apertur und Gesamtschichtdicken von über $100 \mu \mathrm{m}$ hergestellt werden [129]. Aufgrund niedriger Spannungen in $\mathrm{WSi}_{2} / \mathrm{Si}-\mathrm{Filmen}$ konnte bereits die Deposition einer $46 \mu \mathrm{m}$ dicken MLL mit $\Delta r=5 \mathrm{~nm}$ demonstriert werden. Für eine Maximierung der Effizienz wurden „wedged“-MLL-Strukturen ebenfalls auf planaren Substraten getestet [130].

Insgesamt lässt sich festhalten, dass mittels MLL/MZP-Herstellung zwei wesentliche Probleme der konventionellen ZP-Manufaktur umgangen werden können: Kleinste äußere Zonenbreiten, notwendig für Aufösungen im sub-10 nm-Bereich, und hohe Aspektverhältnisse, erforderlich für maximale Effizienzen, können durch multilagenbasierte Optiken ermöglicht werden. Dabei müssen die Materialsysteme neben einem hohen Unterschied im Absorptionskoeffizienten hervorragende Wachstumseigenschaften aufweisen: Stabile Depositionsraten, geringe mechanische Spannungen, niedrige Rauigkeiten sowie Interdiffusion und ausreichende Haftungen sind die physikalischen Eigenschaften der zu benutzenden Systeme, die es sehr gut zu kennen gilt. 


\title{
3 Experimentelle Methoden
}

\author{
Dieses Kapitel beinhaltet eine Übersicht aller zur Probenherstellung und \\ -charakterisierung angewandten Methoden. Hauptaugenmerk wird dabei auf \\ die gepulste Laserdeposition (PLD) und die Fokussierte Ionenstrahltechnik \\ (FIB) gelegt, mit denen sämtliche im Rahmen dieser Arbeit aufgezeigten Trans- \\ missionsoptiken für weiche und harte Röntgenstrahlung hergestellt wurden.
}

\subsection{Gepulste Laserdeposition}

Das Verfahren der gepulsten Laserdeposition (PLD) hat sich seit der Herstellung von Hochtemperatursupraleitern in den 80er Jahren zu einer flexiblen Herstellungsmethode komplexer Schichtsysteme verschiedenster Materialklassen wie Metalle, Halbleiter, Oxide und Polymere etabliert [131]. Experimentell wird dabei ein intensiver Laserstrahl auf das zu ablatierende Material (sog. Target) fokussiert, wodurch die Targetoberfläche innerhalb weniger Nanosekunden bis zu $6000 \mathrm{~K}$ erhitzt und eine gasförmige Knudsenlage gebildet wird [132]. Durch Absorption weiterer Strahlung innerhalb eines Laserpulses entsteht ein teilweise hochgradig ionisiertes Plasma, welches durch Stoßprozesse von Atomen und Ionen senkrecht zur Targetoberfläche expandiert. Schnelle und leichte Elektronen ziehen dabei eine Raumladungszone auf, sodass Ionen mit einer kinetischen Energie bis zu $150 \mathrm{eV}$ beschleunigt werden [132]. Je nach eingekoppelter Energiedichte am Target kann der Anteil der Ionen im Plasma über $90 \%$ betragen [133]. Durch Rekombination treffen die restlichen Teilchen als neutrale Atome mit Energien zwischen 5 und $10 \mathrm{eV}$ auf einem dem Target gegenüber befindlichen Substrat auf. Im Gegensatz zur Sputterdeposition $\left(E_{k i n}=5-10 \mathrm{eV}\right)$ oder zum thermischen Verdampfen $\left(E_{k i n}<1 \mathrm{eV}\right)$ können bei PLD hochenergetische Ionen verstärkt zu einem Absputtern oder einer Implantation in die bereits aufgewachsene Schicht führen, sodass der eigentliche Wachstumsprozess einige Monolagen unterhalb der Schichtoberfläche erfolgt (engl. „subsurface growth“) [134]. Ein weiterer PLD- 


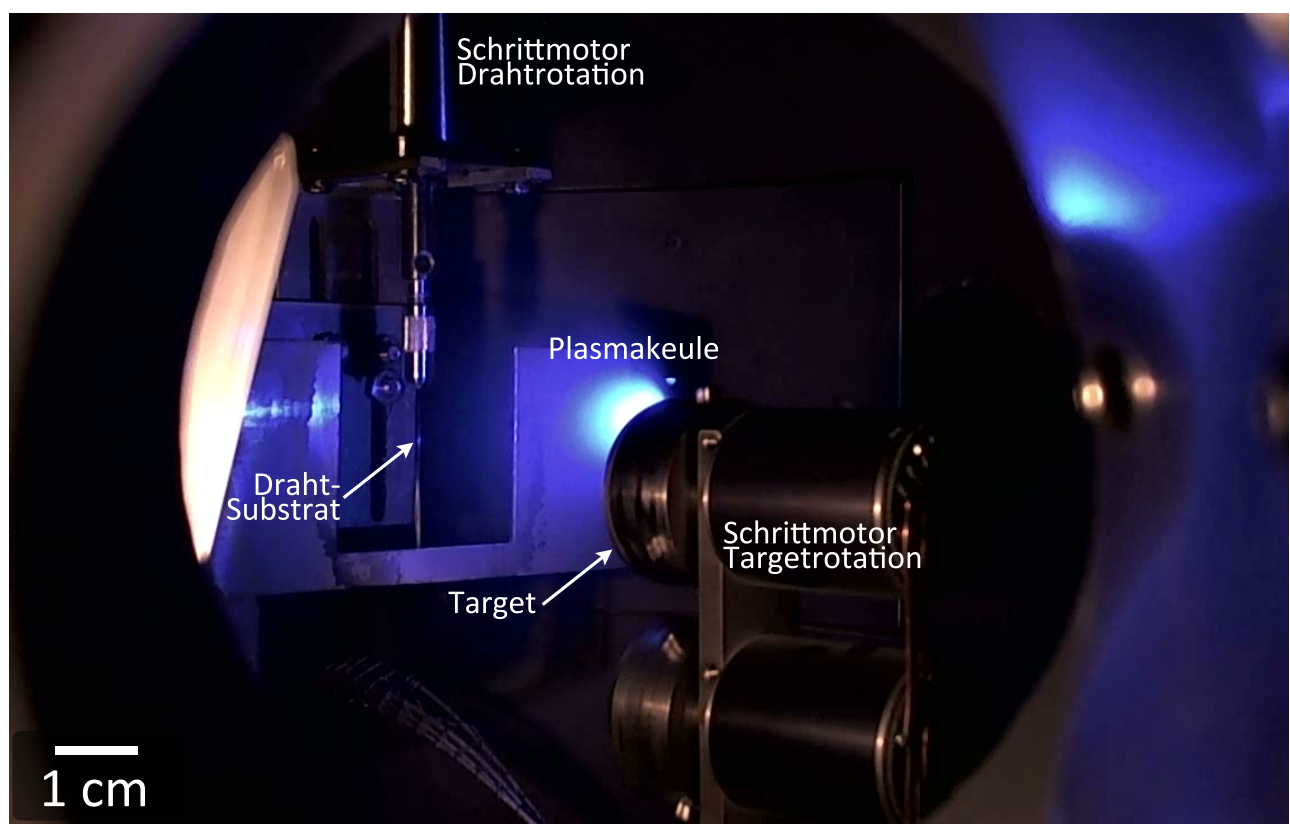

Abbildung 3.1: Blick ins Innere der Depositionskammer während der Multischichtherstellung auf einen rotierenden Draht. Die Plasmakeule gehört zu dem momentan verwendeten $\mathrm{ZrO}_{2}$-Target. Die Richtung des Laserstrahls zeigt in dieser Ansicht aus der Bildebene heraus.

typischer Plasmabestandteil sind makroskopische flüssige Tröpfchen (engl. droplets), welche aufgeschmolzenem Targetmaterial entspringen [135].

Als externe Energiequelle wurde ein KrF-Excimerlaser (LPX 110i, LAMBDA PHYSIK) mit der Wellenlänge $\lambda=248 \mathrm{~nm}$ und einer Pulsdauer von $\tau=30$ ns verwendet, der bei einer Repetitionsrate von $10 \mathrm{~Hz}$ betrieben wurde. Je nach Wahl der Laserenergie und der Strahlfokussierung wurden am Target Energiedichten zwischen 1,3 und $3,3 \mathrm{~J} / \mathrm{cm}^{2}$ erzeugt. Für die Multischichtherstellung können wie in Abbildung 3.1 ersichtlich bis zu drei Targets in den Strahlengang des Lasers eingeführt werden, welche durch Translations- und Rotationsbewegungen (s. dazu Kap. 4.2) flächendeckend und gleichmäßig abgerastert werden. Als Substratmaterialien wurden planare Si(111)- sowie einseitig gedünnte $\mathrm{Si}_{3} \mathrm{~N}_{4}$-Substrate und für die Deposition auf gekrümmten Oberflächen FeC- sowie Glasdrähte unterschiedlicher Durchmesser verwendet. Für die Umsetzung einer Drahtrotation wurde ein neuer UHV-Schrittmotor (VSS 25.200.1.2, PHYTRON) in die Depositionskammer integriert. Die gewählte Umdrehungsgeschwindigkeit ist dabei beliebig, muss jedoch schneller sein als eine Einzelschicht dauern würde, damit jedes Flächenelement des Drahtes gleichmäßig beschichtet werden kann. Mittels einer neuen $x y z$-Verfahreinheit (VAB ELMSHORN), welche im Rahmen der begleiteten Bachelorarbeit von Dirk Fetzer an der Anlage eingesetzt wurde [136], lassen sich verschiedene 
Substrate sowie ein Depositionsratenmonitor (s. Kap. 3.5) in-situ im Materialstrom des Plasmas positionieren. Weiterhin kann auf diese Weise der Target-SubstratAbstand verändert werden, der hier zwischen 4 und $7 \mathrm{~cm}$ gewählt wurde. Während der Schichtherstellung herrschte innerhalb der Vakuumkammer stets ein Basisdruck kleiner als $1 \cdot 10^{-8}$ mbar sowie Raumtemperatur. Zur weiteren Druckminderung wurde teilweise eine Kältefalle mit flüssigem Stickstoff betrieben.

\subsection{Fokussierte Ionenstrahltechnik}

Die Verwendung fokussierter Ionenstrahlen (engl. focused ion beam, FIB) erlaubt Abbildung und Mikromanipulation auf der Nanometerskala in einem einzigen Gerät. Das Hauptanwendungsgebiet einer FIB-Apparatur ist im Wesentlichen in der Probenpräparation für eine Vielzahl analytischer Methoden wie z. B. die Fabrikation von TEM-Lamellen zu sehen [137]. Dabei wechselwirkt ein fokussierter Ionenstrahl mit der Probenoberfläche, der hauptsächlich ein atomares Sputtern der Schicht verursacht und somit eine gezielte Strukturierung ermöglicht. Der Energietransfer bei Stoßprozessen führt außerdem zu Rückstreuung der Ionen, Emission von (Sekundär-) Elektronen sowie elektromagnetischer Strahlung, Ionenimplantation und Aufheizung der Probe [138]. Da emittierte Sekundärelektronen je nach Signalstörung durch gesputterte Teilchen nur begrenzt zur Abbildung genutzt werden können (ca. $10 \mathrm{~nm}$ Auflösung), wird häufig ein zusätzlich integriertes Rasterelektronenmikroskop (REM, s. Kap. 3.6.1) zur Bildgebung verwendet (sog. „dual-beam“-FIB). Durch Einlassen einer gasförmigen $\left(\mathrm{CH}_{3}\right)_{3} \mathrm{Pt}\left(\mathrm{C}_{\mathrm{p}} \mathrm{CH}_{3}\right)$-Verbindung wird bei Abrasterung eines Musters durch den Elektronen- oder Ionenstrahl eine chemische Reaktion hervorgerufen, die eine lokale Pt-Deposition bewirkt. Diese dient einerseits als Schutzfunktion vor einer Ionenkontamination des interessanten Probenbereichs und andererseits als Verbindungsmittel zwischen Schicht und einer feinen Spitze (Mikromanipulator), mit der es möglich ist, vorgeschnittene Segmente aus einer Probe herauszuheben und zur Weiterverarbeitung auf einen Probenhalter zu transferieren.

Das im Rahmen dieser Arbeit verwendete Nova 600 NanoLab (FEI) besteht im Wesentlichen aus einer flüssigen $\mathrm{Ga}^{+}$-Ionenquelle, einem integrierten REM, einem 5-Achsen-Probentisch und einem 3-Achsen-Mikromanipulator (Wolfram-Spitze, Omniprobe). Zum Freilegen und Polieren der Schichtsysteme durch Sputtern wurden für die MLL/MZP-Fabrikation $30 \mathrm{keV} \mathrm{Ga}{ }^{+}$-Ionen mit Strahlströmen zwischen $20 \mathrm{nA}$ und $30 \mathrm{pA}$ genutzt. 


\section{3 „table-top“-Röntgenmikroskopie}

Aufgrund der begrenzten Messzeit an Synchrotron-Strahlungsquellen stellt die lasergetriebene Erzeugung von Röntgenstrahlung eine vielversprechende Möglichkeit dar, die Röntgenmikroskopie unabhängig und frei zugänglich auf Laborgröße zu betreiben [139]. Zu diesem Zweck wird intensive Laserstrahlung mit einer Leistungsdichte von $10^{12}-10^{13} \mathrm{~W} / \mathrm{cm}^{2}$ auf einen konzentrierten Gasfluss, ein flüssiges oder ein Festkörpertarget fokussiert [140] und durch Multiphoton-Ionisationsprozesse ein heißes, dichtes Plasma erzeugt, welches elektromagnetische Strahlung bis hinunter in den Röntgenbereich $(1-20 \mathrm{~nm})$ emittiert $[141,142]$. Speziell bei weicher Röntgenstrahlung sind dabei Plasma-Temperaturen von $T \geq 10^{6} \mathrm{~K}$ sowie Elektronen- und Ionendichten von $10^{19}-10^{20} \mathrm{~cm}^{-3}$ notwendig [32]. Bei den resultierenden Emissionsspektren wird zwischen quasi-kontinuierlicher breitbandiger Strahlung, die mit schweren Targetgasen hoher Ordnungszahl $Z$ wie z. B. Krypton oder Xenon erzeugt wird, und monochromatischer Linienstrahlung unterschieden, die durch leichte Elemente wie Sauerstoff oder Stickstoff zustande kommt.

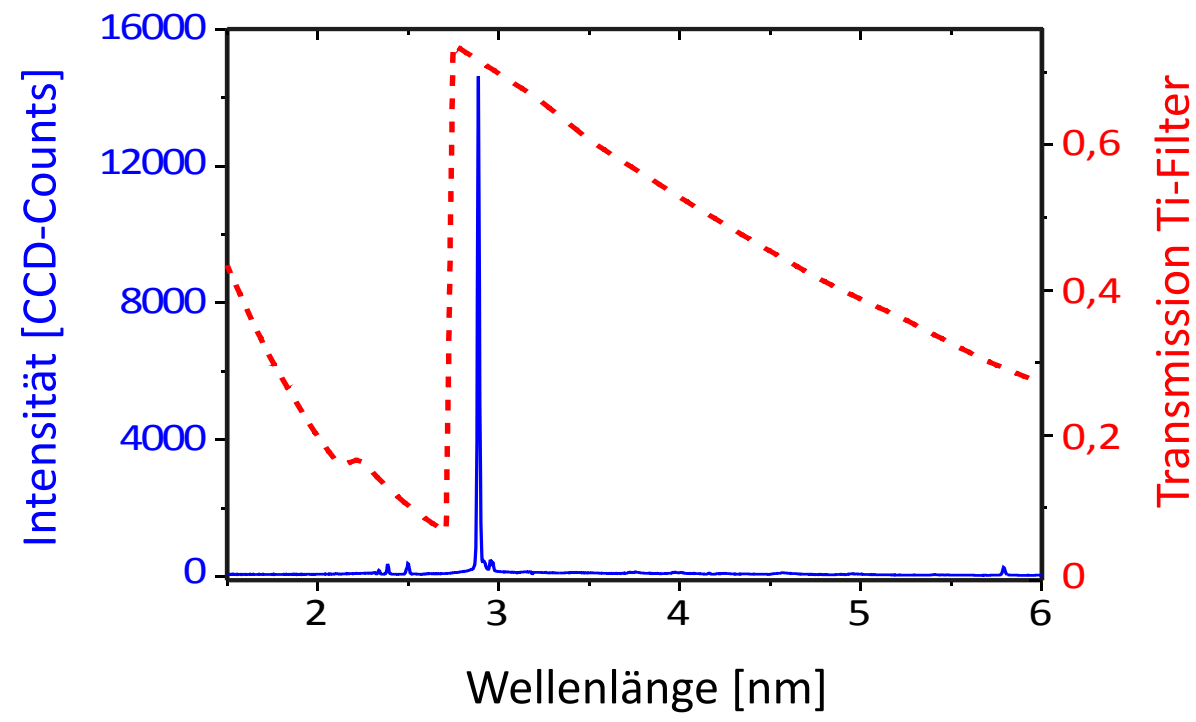

Abbildung 3.2: Mittels einer $200 \mathrm{~nm}$ dicken Ti-Folie gefiltertes Emissionsspektrum der mit Stickstoff betriebenen Plasmaquelle des Laser-Laboratoriums Göttingen. Nur die starke Linienemission bei 2,879 nm wird nicht unterdrückt und kann als monochromatische, weiche Röntgenquelle benutzt werden. (Die Daten wurden freundlicherweise von Michael Reese zur Verfügung gestellt.)

In den von Michael Reese (Laser-Laboratorium Göttingen e.V.) durchgeführten Experimenten wurde gasförmiger Stickstoff als Linienstrahler verwendet, dessen charakteristische Emissionslinie bei $\lambda=2,879 \mathrm{~nm}$ mit Hilfe eines Ti-Filters isoliert wurde 
(s. Abb. 3.2). Aufgrund des relativ großen Abstands der multilagenbasierten Transmissionsoptiken zur Röntgenquelle von $83 \mathrm{~cm}$ wurde eine parallele Beleuchtung mit planaren Wellenfronten approximiert. Die Optiken wurden dabei so im Strahlengang fixiert, dass die kleinere Halbachse des ca. $300 \times 800 \mu \mathrm{m}^{2}$ großen Röntgenplasmas fokussiert wurde. Zur Aufnahme von Beugungsbildern wurde eine rückseitig gedünnte CCD-Kamera (PI-SX: 1k, Roper ScIentific) mit 1024² Pixeln der jeweiligen Größe von $13 \times 13 \mu \mathrm{m}^{2}$ verwendet.

\subsection{Untersuchungen mit Synchrotronstrahlung}

Strahlexperimente bzgl. der Fokuseigenschaften der für harte Röntgenstrahlung entworfenen MLL wurden durch Mitarbeiter des Göttinger Instituts für Röntgenphysik (Sven Philip Krüger, Klaus Giewekemeyer, Markus Osterhoff und Tim Salditt) am Deutschen-Elektronen-Synchrotron DESY, Hamburg, durchgeführt. Am Strahlrohr P10 des Beschleunigerrings PETRA III steht dazu ein hochkohärenter Röntgenstrahl mit einem Photonenfluss von $10^{11}-10^{12} \mathrm{ph} / \mathrm{sec}$ und einer Wellenlänge von $\lambda=0,0898 \mathrm{~nm}(13,8 \mathrm{keV})$ zur Verfügung [143], welcher mit einer Quellgröße von $36 \mu \mathrm{m} \times 6 \mu \mathrm{m}$ (horizontal $\times$ vertikal) zunächst auf eine Strahlgröße zwischen 300 und $400 \mathrm{~nm}$ (je nach Strahlqualität im Beschleunigerring) vorfokussiert wird. Dafür werden zwei elliptisch geformte, mit Palladium beschichtete Totalreflexionsspiegel verwendet, die in einer Kirkpatrick-Baez-Geometrie (KB-Optik) angeordnet sind [144]. Mit einer Fokuslänge von $f_{\mathrm{KB}}=200 \mathrm{~mm}$ ergibt sich innerhalb der Fokusebene eine hohe Schärfentiefe von $200 \mu \mathrm{m}$, in der von einer gaußförmigen Strahlpropagation mit parallelen Wellenfronten ausgegangen werden kann. Hier wurde im zweiten Schritt eine MLL positioniert, die schließlich einen extrem kleinen Nanofokus erzeugen soll. Zur Aufnahme von Beugungsbildern wurde ein Einzelphoton zählender Detektor mit einer Pixelgröße von $173 \times 173 \mu \mathrm{m}^{2}$ (PILATus 300K, Dectris) verwendet, der in 5,39 m Entfernung zur MLL positioniert war.

\subsection{Schichtdickenanalyse}

Die ständige Schichtdickenkontrolle der hergestellten Proben ist für die Vorbereitung einer MLL/MZP-Fabrikation unerlässlich. Zu diesem Zweck wurden verschiedene Methoden angewandt, wobei die Röntgenreflektometrie die höchste quantitative 
Schichtdickenauflösung bietet. Zusätzlich wurden Schichtdicken von präparierten Querschnittslamellen direkt im FIB vermessen.

\subsubsection{Profilometrie}

Bei der Profilometrie wird eine dünne Nadel senkrecht über eine definierte Kante zwischen Schicht und unbeschichtetem Substrat geführt, wobei der resultierende Höhenunterschied als Schichtdicke aufgefasst wird. Die dabei benutzten Si-Substrate wurden vor der Deposition von Einzelschichten mit einem dünnen Kapton-Klebestreifen versehen, um durch späteres Ablösen eine entsprechende Kante zu erzeugen. Zur Messung wurde ein Dektak 150 (VEeco) mit einer theoretischen Aufösung kleiner als $1 \mathrm{~nm}$ verwendet. Experimentell wird diese bei einer Schicht mit hohem Dropletanteil oder einer undefinierten und rauen Kante jedoch stark beeinträchtigt. Zudem muss das zur Referenz dienende Substrat möglichst waagrecht ausgerichtet werden.

\subsubsection{Massenwägung}

Eine sehr sensitive in-situ-Erfassung von Schichtdickenänderungen stellt das Prinzip der Massenwägung dar. Während einer Schichtherstellung wird dazu ein Quarzkristall in eine empfindliche Dickenscherschwingung der Eigenfrequenz $f_{0}$ versetzt, welche sich folglich eines Zuwachses an deponierter Masse verringert. Über die Frequenzänderung $f / f_{0}$ lässt sich auf die Schichtdicke

$$
d_{\mathrm{S}}=-\frac{f}{f_{0}} \frac{\rho_{\mathrm{Q}} d_{\mathrm{Q}}}{\rho_{\mathrm{S}}}
$$

schließen, wobei $\rho_{\mathrm{S}, \mathrm{Q}}$ die Dichten des Schichtmaterials bzw. des Quarzkristalls der Dicke $d_{\mathrm{Q}}$ sind [145]. Zur Messung wurde ein Depositionsratenmonitor SQM 160 (INFICON) in die Vakuumanlage integriert, der im arbeitenden Frequenzbereich zwischen 4 und $6 \mathrm{MHz}$ eine Dickenauflösung von $0,037 \AA$ (bei $1 \mathrm{~g} / \mathrm{cm}^{3}$ ) bietet. Wie in Abbildung 3.3 anhand des zeitlichen Verlaufs der Schichtdicke während der Deposition von 50 Pulsen $\mathrm{ZrO}_{2}$ zu erkennen ist, zeigt der Ratenmonitor eine Relaxationszeit von ca. 30 s, sodass zur Datenerfassung nach Abgabe des letzten Pulses entsprechend gewartet werden muss. Die anfängliche negative Schichtdicke spricht in diesem Fall für ein Absputtern des bereits auf dem Quarzkristall vorhandenen Films. 


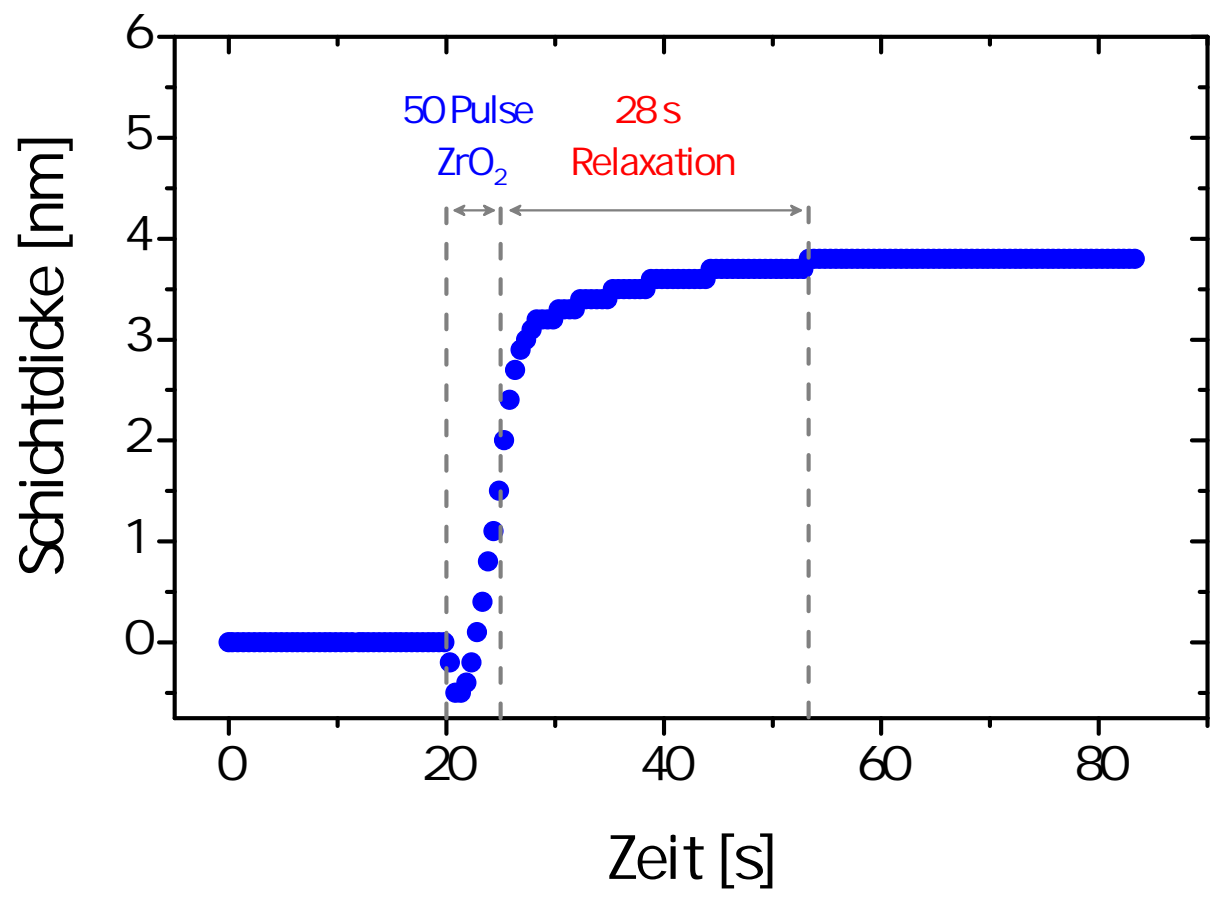

Abbildung 3.3: Einschwingverhalten des Depositionsratenmonitors nach der Abgabe von 50 Laserpulsen $\mathrm{ZrO}_{2}$. Bis zum Erreichen einer im Gleichgewicht befindlichen Schichtdicke muss ca. $30 \mathrm{~s}$ gewartet werden.

\subsubsection{Röntgenreflektometrie}

Die wohl wichtigste Methode zur Bestimmung von Einzel- sowie Multischichten gleicher Periode stellt hier die spekuläre Röntgenreflektometrie (XRR) dar. Mittels harter Röntgenstrahlung wird dazu die reflektierte Intensität eines dünnen Films (bis ca. $300 \mathrm{~nm}$ Dicke) unter sehr kleinen Einfallswinkeln $\left(\theta \approx 0^{\circ}-5^{\circ}\right)$ gemessen, welche Auskunft über das Elektronendichteprofil senkrecht zur Probenoberfläche gibt. Mit dieser Information lassen sich Einzel- und Doppelschichtdicken, Grenz- und Oberflächenrauigkeiten sowie die chemische Zusammensetzung des Films erschließen. Strukturelle oder chemische Rauigkeiten können anhand des integralen Elektronendichteprofils jedoch nicht unterschieden werden. Die Doppelschichtdicke $\Lambda$ berechnet sich anhand der Bragg-Beziehung zu

$$
n \lambda=2 \Lambda \sin \theta
$$

wobei hier die konstruktive Interferenz nicht an den Gitterebenen, wie es bei der Röntgendiffraktometrie (s. Kap. 3.6.2) der Fall ist, sondern an den Grenzflächen der 
Schichten (sog. Übergitter) erfolgt. Durch die Winkellage der Totalreflexionskante, ab der der Röntgenstrahl in die Probe eindringt, sowie das Auftreten oder die Auslöschung von Bragg-Peaks bestimmter Ordnungen eines XRR-Spektrums lassen sich das Schichtdickenverhältnis $\Gamma$ und damit die Einzelschichtdicken mit einer Genauigkeit im Ångströmbereich ermitteln. Bei einem Schichtpaket gleicher Einzelschichtdicke $(\Gamma=0,5)$ werden unter Vernachlässigung der Rauigkeit alle geraden Bragg-Peaks aufgrund destruktiver Interferenz ausgeblendet [146], wie es in Abbildung 3.4 anhand von Multischicht-Simulationen ähnlicher Schichtdickenverhältnisse deutlich wird.

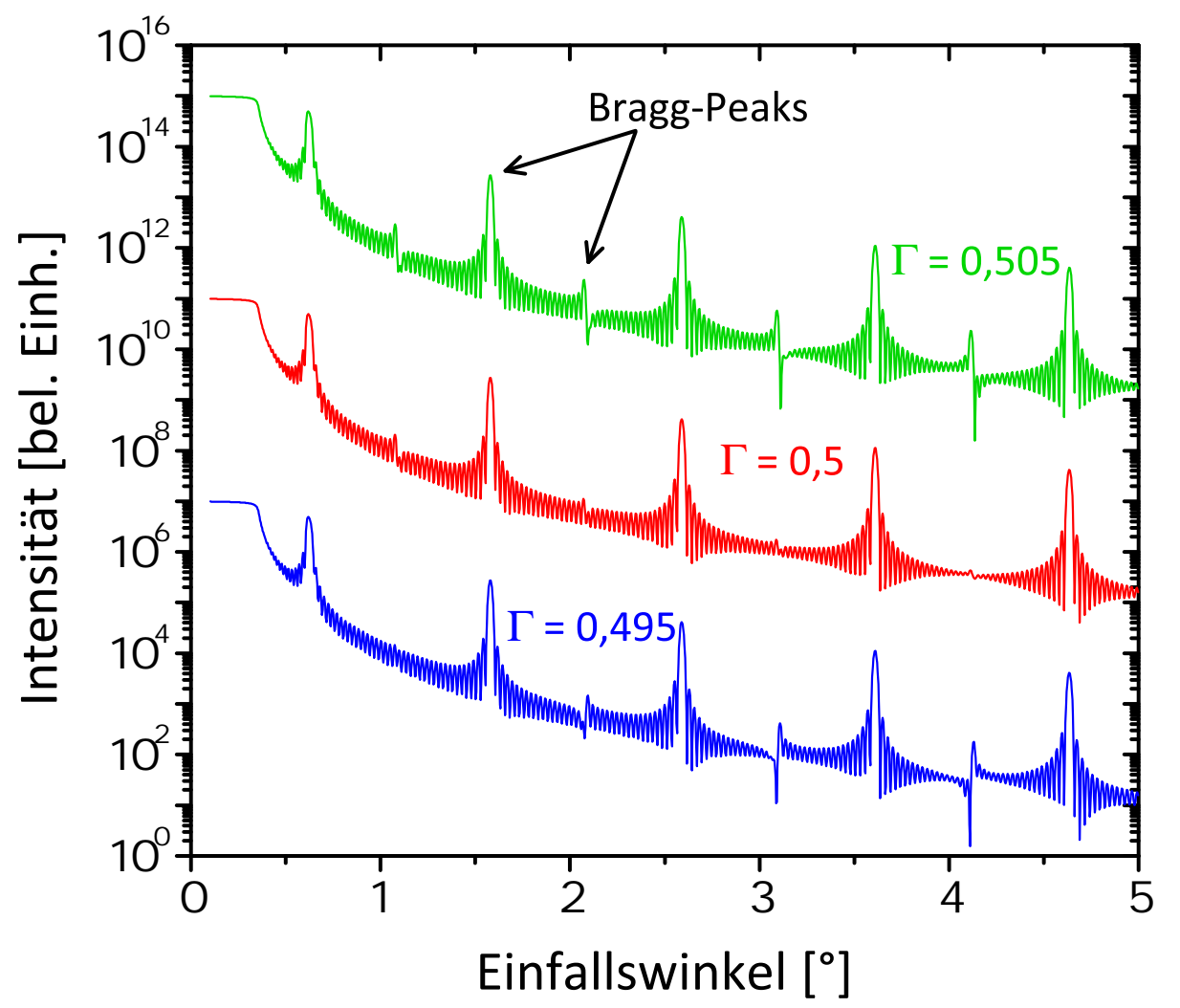

Abbildung 3.4: Simulierte XRR-Spektren eines $\mathrm{MgO} / \mathrm{ZrO}_{2}-$ Schichtpakets mit jeweils 10 Doppelschichten und unterschiedlichen Schichtdickenverhältnissen $\Gamma$. Bereits geringe Änderungen der Einzelschichtdicken von $\pm 1 \%$ ( $\Gamma=0,495$ bzw. 0,505) lassen Bragg-Peaks gerader Ordnung durch konstruktive Interferenz auftreten, die bei $\Gamma=0,5$ unter Vernachlässigung von Rauigkeiten nahezu vollständig ausgelöscht sind.

Zur Erfassung von XRR-Spektren wurde ein Vierkreisdiffraktometer X'Pert (PhILIPS) mit einer Wellenlänge von $\lambda=1,79026 \AA$ (Co-Röntgenröhre) benutzt. Die Simulation der Spektren wurde mit dem Softwarepaket IMD/XOP [147] durchgeführt, welches auf den rekursiven Parratt-Algorithmus zurückgreift [148]. Detaillierte Beschreibungen sind in früheren Arbeiten ausgeführt [149]. 


\subsubsection{Transmissionselektronenmikroskopie}

Besonders zur Schichtdicken- aber auch zur Strukturanalyse (s. Kap. 3.6) wurde die Transmissionselektronenmikroskopie (TEM) verwendet, bei der die Wechselwirkung von Elektronenstrahl und einer gedünnten Probe in Transmission stattfindet [150]. Zur Bildgebung werden hauptsächlich elastisch gestreute Primärelektronen detektiert. Um kontrastreiche Aufnahmen zu erzeugen, wird eine optische Tiefe der mittels FIB präparierten Querschnittslamellen (s. Kap. 3.2) kleiner als $100 \mathrm{~nm}$ vorausgesetzt. Durch Änderung des Strahlengangs können Real- oder Beugungsbilder der Probe generiert werden, die hier mit einem CM 30 (PHILIPS) aufgenommen wurden.

\subsection{Strukturanalyse}

Mit den folgenden Methoden wurden strukturelle Untersuchungen bzgl. Oberflächenmorphologie, kristallinem Wachstum sowie Stöchiometrieübertrag vorgenommen.

\subsubsection{Rasterelektronenmikroskopie}

Um die Oberflächenmorphologie von Proben und Targets zu untersuchen, wurde die Rasterelektronenmikroskopie (REM) verwendet, bei der ein fokussierter Elektronenstrahl die Oberfläche abrastert. Die Wechselwirkung zwischen Strahl und Probe erzeugt rückgestreute Primärelektronen (elastische Streuung) sowie die Emission von Sekundärelektronen (inelastischer Prozess) [151]. Intensität und Austrittsrichtung der Sekundärelektronen lassen einen morphologischen Kontrast zu, welcher häufig zur Bildgebung genutzt wird. Neben dem integrierten REM im FIB (s. Kap. 3.2) wurden außerdem folgende Geräte verwendet: S 360 und Supra 35 (LEO, ehemals CAMBRIDGE Instruments) sowie Nova NanoSEM 650 (FEI). Beim S 360 wurde die Emission von Sekundärelektronen weiterhin genutzt, um eine energiedispersive Röntgenfluoreszenzanalyse (engl. energy dispersive x-ray analysis, EDX) durchzuführen. Diese ermöglicht eine elementspezifische Konzentrationsanalyse.

\subsubsection{Röntgendiffraktometrie}

Zur strukturellen Untersuchung kristalliner Materialien bietet sich die Methode der Röntgendiffraktometrie (XRD) an, bei der die einfallende Röntgenstrahlung an unter- 
schiedlichen Netzebenen gestreut wird und somit Aussagen über die Orientierung der Kristallite ermöglicht. Ferner lässt sich anhand der Scherrer-Formel eine quantitative Abschätzung der Korngröße

$$
d=\frac{0,9 \lambda}{\Delta(2 \theta) \cos \theta}
$$

vornehmen, wobei $\Delta(2 \theta)$ der Halbwertsbreite des entsprechenden kristallinen Reflexes entspricht. Als Messinstrument diente ebenfalls das Vierkreisdiffraktometer X'Pert (s. Kap. 3.5.3), welches in üblicher Bragg-Brentano-Geometrie betrieben wurde [152].

\subsubsection{EUV-Reflektometrie}

Die Reflektometrie im extrem ultravioletten Wellenlängenbereich (EUVR) zeichnet sich vor allem dadurch aus, dass die EUVR-Spektren aufgrund der relativ großen Wellenlänge über einen großen Winkelbereich ausgedehnt sind und somit im Vergleich zur konventionellen XRR mit harter Röntgenstrahlung (s. Kap 3.5.3) eine bessere Auflösung bieten. Durch die hohe Wechselwirkung mit Materie aufgrund hoher Absorption ist diese Analysemethode zudem sehr sensitiv bezüglich der Bestimmung von Rauigkeit, Dichte, komplexem Brechungsindex und chemischer Komposition der zu untersuchenden Materialien [153].

Die Messungen und zugehörige Simulationen wurden von Stefan Döring im LaserLaboratorium Göttingen e. V. mittels eines EUV-Reflektometers mit einer lasergetriebenen Plasmaquelle (s. Kap. 3.3) an der $\mathrm{O}_{2}$-Absorptionskante durchgeführt. Die emittierte Röntgenstrahlung kann durch ein anpassungsfähiges Kirkpatrick-BaezSpiegelsystem kollimiert oder fokussiert auf die Probe gelenkt werden [140]. Spektral gefiltert ergibt sich ein quasi-monochromatischer Röntgenstrahl mit 12,98 nm Wellenlänge, der eine Größe von ca. $300 \times 1000 \mu \mathrm{m}^{2}$ einnimmt. Das in der Probenkammer befindliche Goniometer ist frei rotierbar und wurde im $\theta-2 \theta$-Modus betrieben. 


\title{
4 Optimierung zur Herstellung hochpräziser Multischichtstrukturen
}

\author{
Nachfolgend sollen die im Rahmen dieser Arbeit durchgeführten notwendigen \\ Prozessoptimierungen vorgestellt werden, um Röntgenoptiken mit hochpräzisen \\ aperiodischen Schichtdicken herstellen zu können. Wesentliche Ziele sind dabei \\ die Stabilität der Depositionsraten zu optimieren sowie auf Veränderungen \\ der Raten durch entsprechende Gegenmaßnahmen reagieren zu können. Dabei \\ wurden Hard- und Software der PLD-Anlage maßgeblich verbessert.
}

Neben einer schwierigen Software-Implementierung der in den Herstellungsprozess neu integrierten Geräte und einer unzureichenden Flexibilität des existierenden Laserprogramms war die Tatsache, dass lediglich periodische Schichtdicken realisiert werden konnten, der entscheidende Grund für die Entwicklung einer komplett neuen Steuerung der Depositionsanlage. Diese wurde mit der graphischen Programmierumgebung LabVIEW 2009 von National Instruments umgesetzt. Durch die modulare Programmstruktur von LabVIEW lässt sich auf intuitive Weise über verschiedenste Schnittstellen mit Hardware-Controllern kommunizieren sowie die Datenerfassung von Messgeräten, deren Weiterverarbeitung und Speicherung ausführen [154]. Sämtliche hier benötigten Geräte wie Laser, Oszilloskop, Depositionsratenmonitor und eine Vielzahl an Schrittmotoren werden durch Programmblöcke (sog. virtuelle Instrumente) separat angesteuert und ausgelesen, welche in übergeordneten Programmen des eigentlichen Herstellungsprozesses synchron oder nacheinander ausgeführt werden können. Die vollständig automatisierte Anlagensteuerung ist auf jede der drei vorhandenen Depositionskammern spezifisch angepasst und ermöglicht eine flexible Übergabe frei konfigurierbarer Herstellungsparameter für beliebig viele Targets, umfangreiche Justagemöglichkeiten sowie eine ausführliche Dokumentation über den Herstellungsverlauf. In Abbildung 4.1 ist die Benutzerschnittstelle der im Rahmen dieser Arbeit verwendeten Depositionskammer gezeigt, mit der dem Anwender viele Möglichkeiten 


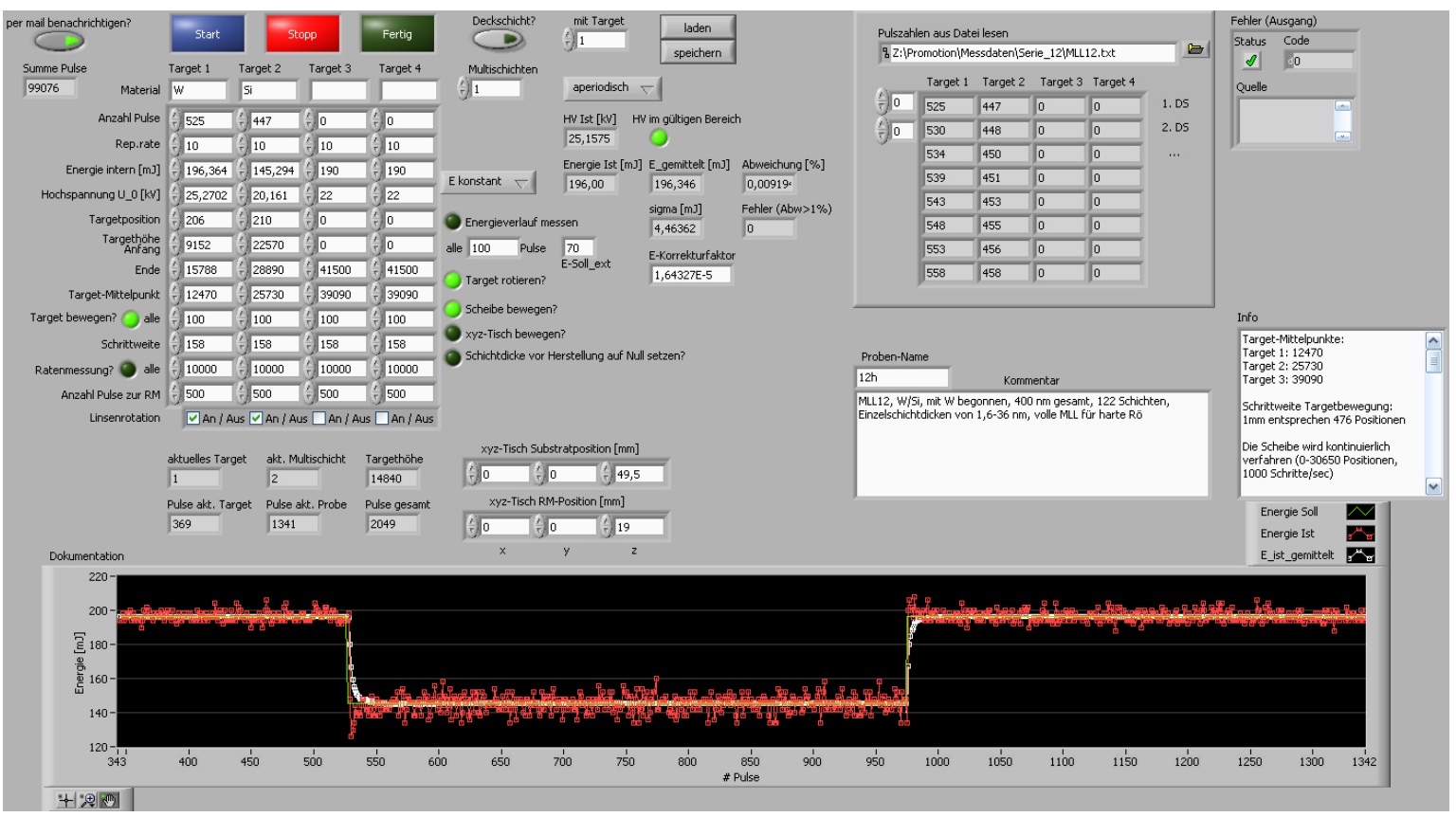

Abbildung 4.1: Benutzeroberfläche des neu entwickelten Laserprogramms. Neben zahlreichen einstellbaren Herstellungsparametern und graphischer Kontrolle der ausgegebenen Laserenergie ist nun die Deposition aperiodischer Multischichten möglich.

zur komplexen Herstellung periodischer und aperiodischer (Multi-)Schichtstrukturen eröffnet werden.

\subsection{Stabilisierung der Energiedichte am Target}

$\mathrm{Zu}$ Beginn dieser Arbeit war es nicht möglich, vorgegebene Schichtdicken größer als $1 \mu \mathrm{m}$ mit einer geringen Abweichung $(<5 \%)$ herzustellen. Der Grund hierfür lag an einem starken Verlust der eingekoppelten Energiedichte am Target während der Schichtherstellung. Für eine Langzeitstabilität der Energiedichte wurden daher die im Folgenden gezeigten Veränderungen am Herstellungsprozess vorgenommen: 1. eine neue Energieregelung des Lasers und 2. eine Erhöhung des Abstands zwischen dem Eintrittsfenster und dem eigentlichen Depositionszentrum innerhalb der Vakuumkammer.

\subsubsection{Energieregelung des Lasers}

Während einer Schichtherstellung ist es besonders wichtig, Laserstrahlung mit einer möglichst konstanten Energie zu verwenden. Da dem Laser nur Hochspannungswerte 
übergeben werden, erfolgt die eigentliche Energieregelung über das Laserprogramm mittels Abfrage des internen Laser-Energiemonitors nach jedem abgegebenen Puls. Hierbei sind folgende Effekte des Lasers zu berücksichtigen:

- Die Laserenergie streut zunehmend bei niedrigen Hochspannungen und hohen Repetitionsraten.

- Der interne Energiemonitor weist eine starke Streuung bis zu $\pm 11 \%$ auf.

- Die ausgelesene Energie ist nicht die des aktuell abgegebenen Pulses, sondern die des vorangegangenen. Die Energie des ersten Pulses ist also immer Null.

- Die Hochspannung des Lasers, die von $18-26 \mathrm{kV}$ reicht, kann nur mit einer Schrittweite von $\pm 0,1 \mathrm{kV}$ geändert werden.

- Der Wechsel von sehr hoher zu niedriger Hochspannung (z. B. beim Targetwechsel) ist nur über min. 2 Pulse möglich, da der Kondensator erst nach und nach entladen werden kann.

Um trotz dieser nachteiligen Lasereigenschaften mit konstanten Bedingungen arbeiten zu können, musste die Energieregelung des Lasers komplett überarbeitet werden. Hierzu wurde eine PI-(Proportional-Integral-)Regelung programmiert, mit der pro Puls ein neuer Hochspannungswert an den Laser übergeben wird:

$$
U_{\text {neu }}=U_{\text {alt }}+I \Delta E+P \Delta E
$$

$I$ und $P$ sind dabei Konstanten, die die Gewichtung von Integral- und Proportionalteil bestimmen und $\Delta E$ bildet die Differenz zwischen Soll- und Ist-Energie des internen Energiemonitors. Da die ausgelesene Energie des ersten Laserpulses immer Null ist, wird in diesem Fall $E_{\text {Ist }}=E_{\text {Soll }}$ gesetzt. Trotz der extremen Streuung gelingt es mit dieser Regelung bereits nach ca. 100 abgegebenen Laserpulsen einen sehr konstanten Mittelwert der Energie mit einer minimalen Abweichung von $\left|<E_{\text {Ist }}>-E_{\text {Soll }}\right| / E_{\text {Soll }}<0,1 \%$ zu erzielen.

Damit schon zu Beginn der Herstellung immer die gleiche Energiedichte am Target herrscht, muss der erste zu übergebende Hochspannungswert mittels einer vorangehenden Kalibrierung sehr genau bestimmt werden. Hierfür wird bei vorgegebener externer Soll-Energie die zugehörige interne Energie sowie die Hochspannung über einen Bereich einiger 100 Laserpulse mit der oben beschriebenen PI-Regelung aufgenommen und deren konstante Mittelwerte dem eigentlichen Herstellungsprogramm übergeben. So 
können auch hier je nach frei wählbarer Dauer der Kalibrierung minimale Abweichungen kleiner als 0,1\% erreicht werden. Bei dem hier gezeigten Beispiel wurde bereits nach 500 Pulsen eine Abweichung von 0,04\% zur externen Soll-Energie $\left(E_{\text {ext. }}=80 \mathrm{~mJ}\right)$ erreicht (s. Abb. 4.2). Die Hochspannung wurde dazu selbständig zwischen 22,6 und $22,9 \mathrm{kV}$ variiert. Sollen verschiedene Soll-Energien für unterschiedliche Targets verwendet werden, muss die Kalibrierung entsprechend wiederholt werden.

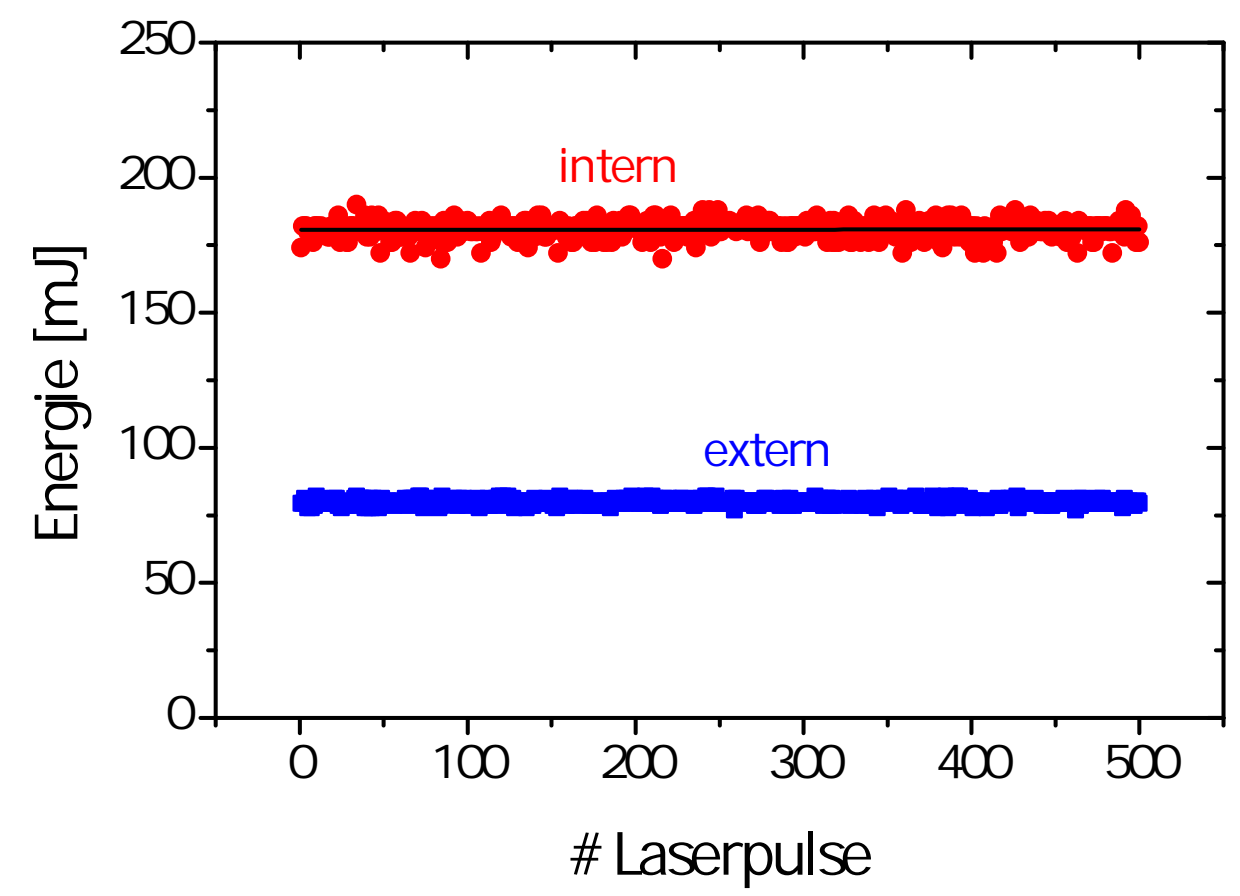

Abbildung 4.2: Kalibrierungsmethode des Lasers vor der Schichtherstellung. Bei einer festen externen Soll-Energie werden die interne Energie sowie die benötigte Hochspannung über mehrere Laserpulse ausgelesen und dem Herstellungsprogramm als Startwerte übergeben (vgl. Abb. 4.1).

\subsubsection{Verlängerung des Strahlengangs im Vakuum}

Ein konstanter Energietransfer des extern befindlichen Lasers in die Vakuumanlage stellt ein gewisses Problem dar, zumal das bei anhaltender Herstellung nach und nach mitbeschichtete Eintrittsfenster der Depositionskammer die Energieeinkopplung verringert. Beim früheren Aufbau der Anlage befand sich das Eintrittsfenster ca. $20 \mathrm{~cm}$ von dem Target entfernt. Dazwischen war eine $2,5 \times 7 \mathrm{~cm}^{2}$ große Schutzscheibe positioniert, die während der Herstellung in diskreten Abständen verfahren wurde, um die Verschmutzung des Eintrittsfensters durch Materialbeschichtung zu verringern. In Abbildung 4.3 unten ist die Transmission von Laserstrahlung in die Vakuumkammer 
in Abhängigkeit der Laserpulse auf Fe bei fester Scheibenposition aufgetragen. Dabei zeigt sich bereits nach ca. $1 \mu \mathrm{m}$ deponierter Schichtdicke auf einem Substrat ein exponentieller Abfall bis um 17\% nach dem Lambert-Beerschen Gesetz

$$
\frac{I}{I_{0}}=e^{-\mu d}
$$

wobei $\mu$ der Absorptionskoeffizient und $d$ die (ungewollte) Schichtdicke, in diesem Fall von Fe, auf der Schutzscheibe ist. Mit diesem viel zu hohen Wert war es faktisch unmöglich, die Herstellung hochpräziser Schichtdicken realisieren zu können.

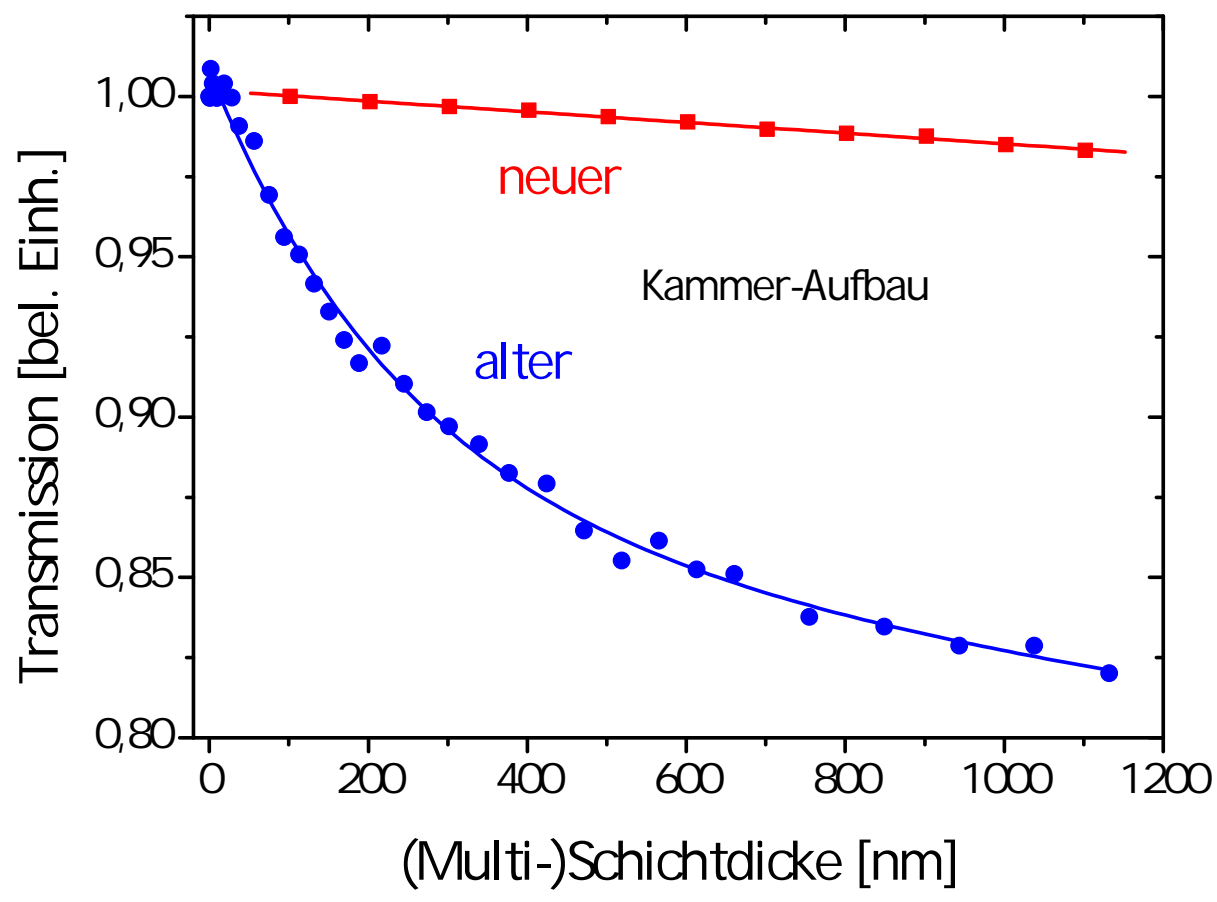

Abbildung 4.3: Unten: Intensitätsverlust der Laserstrahlung innerhalb der Depositionskammer bei alter Kammer-Geometrie während der Deposition von Fe. Durch kontinuierliche Beschichtung der zu nahe am Depositionszentrum positionierten Schutzscheibe fällt die Energiedichte am Target bereits nach ca. $1 \mu \mathrm{m}$ Schichtdicke um 17\% ab. Oben: Transmission der eingestrahlten Energiedichte während einer Multischichtherstellung von $\mathrm{MgO} / \mathrm{ZrO}_{2}$ bei neuer Kammer-Geometrie. Nach $1 \mu \mathrm{m}$ Schichtdicke stellt sich lediglich ein Verlust von $1,5 \%$ ein.

Da diese Beschichtung quadratisch mit dem Abstand zwischen Scheibe und Target abfällt, lag es nahe, das Eintrittsfenster samt der Schutzscheibe weiter nach außen vom Zentrum der Deposition zu setzen. Durch Verwendung einer neuen Fokussierungslinse einer größeren Brennweite $(f=70 \mathrm{~cm})$ konnte diesbezüglich der Strahlengang innerhalb der Vakuumkammer um ca. $50 \mathrm{~cm}$ erweitert werden. Zudem wurde eine neue $3 \times 15 \mathrm{~cm}^{2}$ große Schutzscheibe mit einer kontinuierlichen Bewegung verwendet, um die 
ungewollte Beschichtung auf einer größeren Fläche zu verteilen. Abbildung 4.3 oben zeigt schließlich die Transmission bei einer mittleren Scheibenposition, die während der Herstellung einer periodischen $\mathrm{MgO} / \mathrm{ZrO}_{2}-$ Multischicht aufgenommen wurde. Der minimale Abfall der Transmission wurde mit 1,5\% pro $1 \mu \mathrm{m}$ Multischichtdicke gemessen und konnte somit zugunsten einer möglichst konstanten Energiedichte am Target drastisch reduziert werden. Durch eine zusätzliche, mit dem Laserprogramm durchführbare Energiekorrektur, wie es teilweise bei der Schichtherstellung angewandt wurde, kann der Verlust der Energiedichte am Target weiterhin verringert werden.

\subsection{Homogenisierung des Targetabtrags}

Selbst bei konstanter Energieeinkopplung verringert sich die Depositionsrate im Laufe der Zeit aufgrund einer Änderung der Oberflächenmorphologie des Targets (sog. Targetalterung). Bei anhaltender Laserbestrahlung unter konstantem Winkel stellt sich ein aufrauender Prozess ein, bei dem besonders bei Metallen Zapfen entstehen, die dem einfallenden Laserstrahl entgegengerichtet sind [155]. Die effektive Targetoberfläche vergrößert sich, sodass eine resultierende verringerte Energiedichte zu einer niedrigeren Depositionsrate und einer begünstigten Dropletbildung führt [156]. Eine solche Zapfenbildung kann durch die Verwendung einer Targetrotation verhindert werden, indem die Targetoberfläche von zwei entgegengesetzten Richtungen bestrahlt wird. Diese Abrasterung geschieht wie in Abbildung 4.4 veranschaulicht durch Rotation und Verschiebung des Targets in der Höhe einmal oberhalb und einmal unterhalb des Mittelpunktes.

Zur experimentellen Realisierung ist ein Dreifach-Targethalter mit drei UHVSchrittmotoren (PRECIstep AM2224, FAulHABER) einer Schrittweite von 1/48 pro Vollschritt in die Vakuumkammer integriert worden. Die Rotation in Verbindung mit der Höhenbewegung ist so programmiert, dass auf eine Targetscheibe mit $23 \mathrm{~mm}$ Durchmesser 30 Ringe mit einem Unterschied im Radius von $\Delta r=0,33 \mathrm{~mm}$ abgerastert werden können. Auf diese Weise entsteht eine Überlappung des Laserbrennflecks pro Ring größer als 50\%, sodass ein flächendeckender Abtrag gewährleistet ist. Zur gleichmäßigen Abrasterung der gesamten Targetfläche werden die Laserpulse aufgrund des zunehmenden Umfangs der einzelnen Ringe mit steigendem Radius linear verteilt.

In Abbildung 4.5 lässt sich die enorme Verringerung einer Aufrauung der Targetoberfläche am Beispiel von $\mathrm{ZrO}_{2}$ erkennen. Jeweils links zeigt sich eine wellenartige Struktur, 


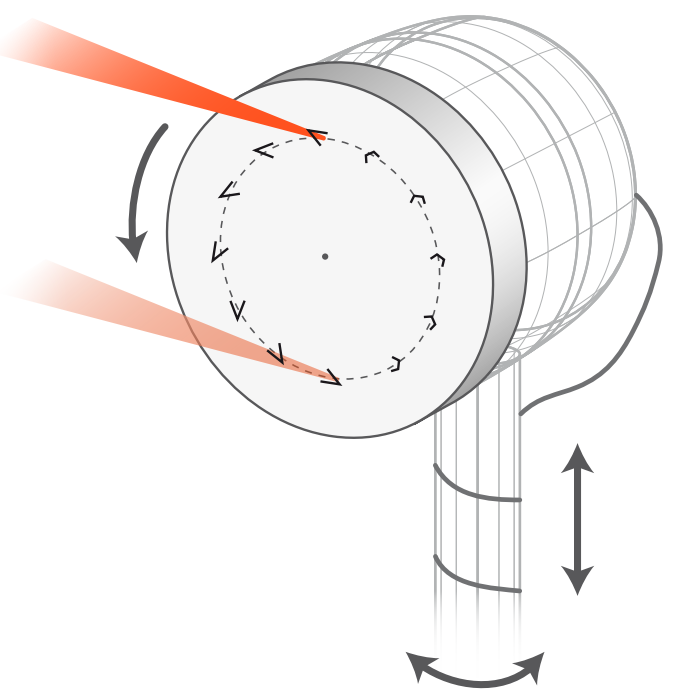

Abbildung 4.4: Schematische Darstellung der Targetrotation. Jedes Flächenelement wird ober- und unterhalb des Target-Mittelpunktes und somit von entgegengesetzten Richtungen bestrahlt. Dadurch wird eine Aufrauung der Oberflächenmorphologie verringert, was eine stabilere Depositionsrate sowie eine geringere Dropletbildung zur Folge hat. Durch Drehung des kompletten Halters kann die Plasmakeule mittig auf das Substrat ausgerichtet werden.
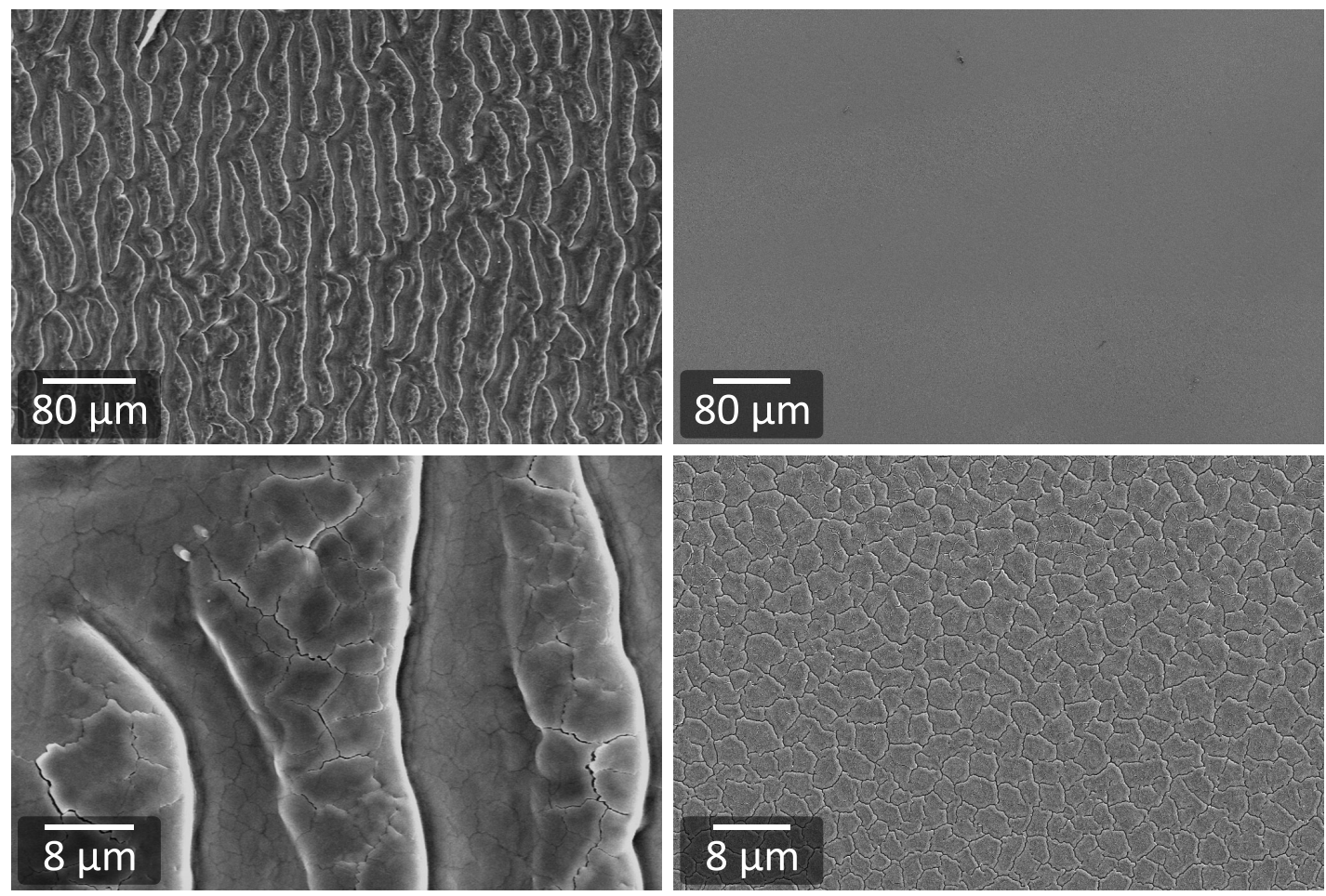

Abbildung 4.5: Vergleich der Oberflächenmorphologien von $\mathrm{ZrO}_{2}$ nach Laserbestrahlung ohne (links) und mit Targetrotation (rechts). Raue Wellenstrukturen, die sich bei einseitiger Abrasterung bilden, werden durch die rotierende Bewegung des Targets komplett unterdrückt. 
die bei einseitiger Bestrahlung durch den Laser auftritt. Hier wurde das Target nach früherem Aufbau lediglich in der Höhe verfahren. Auf der rechten Seite kann diese Aufrauung durch eine zusätzliche Rotation vollständig unterbunden werden, sodass hier nur materialspezifische Spannungsrisse (vgl. Abb. 5.8 in Kap. 5.1.2) zu erkennen sind. Die Homogenisierung des Targetabtrags führt somit zu einer stabileren Depositionsrate. Würde sich die Alterung bei anderen Targets schneller vollziehen, könnte dies durch eine Erhöhung der Laserpulszahl zum Erreichen einer bestimmten Schichtdicke korrigiert werden. Des Weiteren konnte durch die Realisierung der Targetrotation bei der Schichtherstellung von $\mathrm{Cu}$ die Anzahl großer Droplets um das 1,5-fache und die von kleinen um das 2,6-fache reduziert werden (Zur Klassifizierung von Droplets siehe ebenfalls Kap. 5.1.2).

Mit Hilfe all dieser im Laufe der Arbeit durchgeführten Optimierungen wurde es schließlich möglich, hochpräzise Schichtdicken zu realisieren, wie es in Kapitel 5.2 am Beispiel verschiedener MLL-Strukturen gezeigt ist. Nur wenn die Abnahme der Depositionsrate über einen längeren Zeitraum bekannt ist, können entsprechende Gegenmaßnahmen wie z. B. eine Erhöhung der Energie oder eine Erhöhung der Laserpulszahl ergriffenen werden, um die Auswirkungen einer verringerten Energieeinkopplung und einer Targetalterung so gering wie möglich zu halten. 


\title{
5 Transmissionsoptiken für weiche Röntgenstrahlung
}

\begin{abstract}
Mit dem im Rahmen dieser Arbeit neu entwickelten Verfahren zur Fabrikation von Transmissionsoptiken lassen sich Multilagen-Laue-Linsen (MLL) sowohl für weiche als auch harte Röntgenstrahlung realisieren, wobei je nach Wellenlängenbereich unterschiedliche Anforderungen an das Design sowie die Herstellung mittels gepulster Laserdeposition und fokussierter Ionenstrahltechnik gestellt werden. Im Folgenden sollen alternative Materialsysteme für weiche Röntgenstrahlung vorgestellt, verschiedene Geometrien der daraus entstehenden Röntgenoptiken beschrieben und die einzelnen Prozessschritte während der Fabrikation von Multilagen-Laue-Linsen aufgeführt werden. Zusätzlich werden die Fokuseigenschaften einiger Multilagen-Laue-Linsen, vermessen von M. Reese mit einer Labor-Röntgenquelle, dargelegt. Weitere Fokussierungsoptiken sowie erste Demonstratoren von Multilagen-Zonenplatten (MZP), die unter optimierten Bedingungen laserdeponiert wurden, werden im Anschluss vorgestellt.
\end{abstract}

\subsection{Teststruktur einer Multilagen-Laue-Linse}

Bei der Präparation einer MLL ist für die Einhaltung der konstruktiven Interferenz der Einzelschichten die Erfüllung zweier Bedingungen essentiell: Zum einen darf bei der Multischichtherstellung keine kumulative sowie hohe intrinsische Rauigkeit auftreten, und zum anderen bei der Fabrikation einer Linse die akkurate Schichtstruktur nicht gestört werden. Besonders bei der in der Literatur meist verwendeten Sputterdeposition ist es äußerst schwierig, entsprechende Herstellungsparameter zu finden, bei denen keine kumulative Rauigkeit entsteht [111]. Am Beispiel von gesputterten $\mathrm{Cr} / \mathrm{Ti}$-Multischichtspiegeln für weiche Röntgenstrahlung wird die Entstehung einer solchen additiven Rauigkeit durch eine aufwändige modulare Ionenbestrahlung gering 
gehalten [112]. Bei der herkömmlichen Fabrikationsmethode wird die MLL durch Sägen und Polieren hergestellt, wodurch Schäden der Multischichtstruktur entstehen können, die die Verwendung als Röntgenlinse unbrauchbar machen [121]. Besonders beim Einsatz für weiche Röntgenstrahlung müssen die Linsen aufgrund der niedrigen Eindringtiefe sehr dünn und somit technisch anspruchsvoller poliert werden, um den Intensitätsverlust während der Transmission gering zu halten. Das Ziel der in diesem Kapitel aufgezeigten Experimente ist daher, MLL mit einer völlig neuen alternativen Kombination aus PLD und FIB herzustellen, um die hier beschriebenen Problematiken zu umgehen. Darüber hinaus sind in der Literatur bislang keine MLL für die Mikroskopie mit weicher Röntgenstrahlung hergestellt worden, sodass die hier aufgezeigten Optiken eine innovative Weiterentwicklung der aktuellen Forschung darstellen.

\subsubsection{Systemauswahl und Design}

Den wichtigsten Parameter für die Auswahl des Materialsystems einer MLL stellt im Wesentlichen die Röntgenwellenlänge dar, mit der im Einsatzgebiet der Optik gearbeitet werden soll. Die in diesem Kapitel beschriebenen MLL für weiche Röntgenstrahlung sind so konzipiert worden, dass sie in Zusammenarbeit mit dem LaserLaboratorium Göttingen e.V. in einem „table-top“- (d.h. laborgroßen) Röntgenmikroskop (s. Kap. 3.3) mit einer lasergetriebenen Plasmaquelle der Wellenlänge $\lambda=2,879 \mathrm{~nm}$ verwendet werden können [157]. Diese weiche Röntgenwellenlänge befindet sich im sog. „Wasserfenster“, welches durch die Absorptionskanten von Sauerstoff bei 2,3 nm und Kohlenstoff bei 4,4 nm begrenzt wird (s. Abb. 5.1) und sich zur mikroskopischen Analyse von biologischen Zellen innerhalb ihrer fluiden Umgebung eignet. Nahe der Sauerstoff-Kante ergeben sich die besten Bedingungen für eine kontrastreiche Abbildung, bei der Wasserschichten bis zu einer Tiefe von $10 \mu \mathrm{m}$ durchstrahlbar sind, Kohlenstoff hingegen als Hauptbestandteil organischer Zellsysteme die einfallende Strahlung ausreichend absorbiert, sodass sich ein natürlicher Amplitudenkontrast einstellt.

Die Materialauswahl muss also in erster Linie anhand ihrer optischen Eigenschaften bei gegebener Wellenlänge getroffen werden, bei der ein hoher Unterschied im Absorptionskoeffizient $\beta$ der Einzelkomponenten gefordert wird. Bei multilagenbasierten Optiken ist außerdem das Schichtwachstum der Materialien zu berücksichtigen, die bezüglich maximaler Effizienz möglichst glatt und nicht mit einer kumulativen Rauig- 


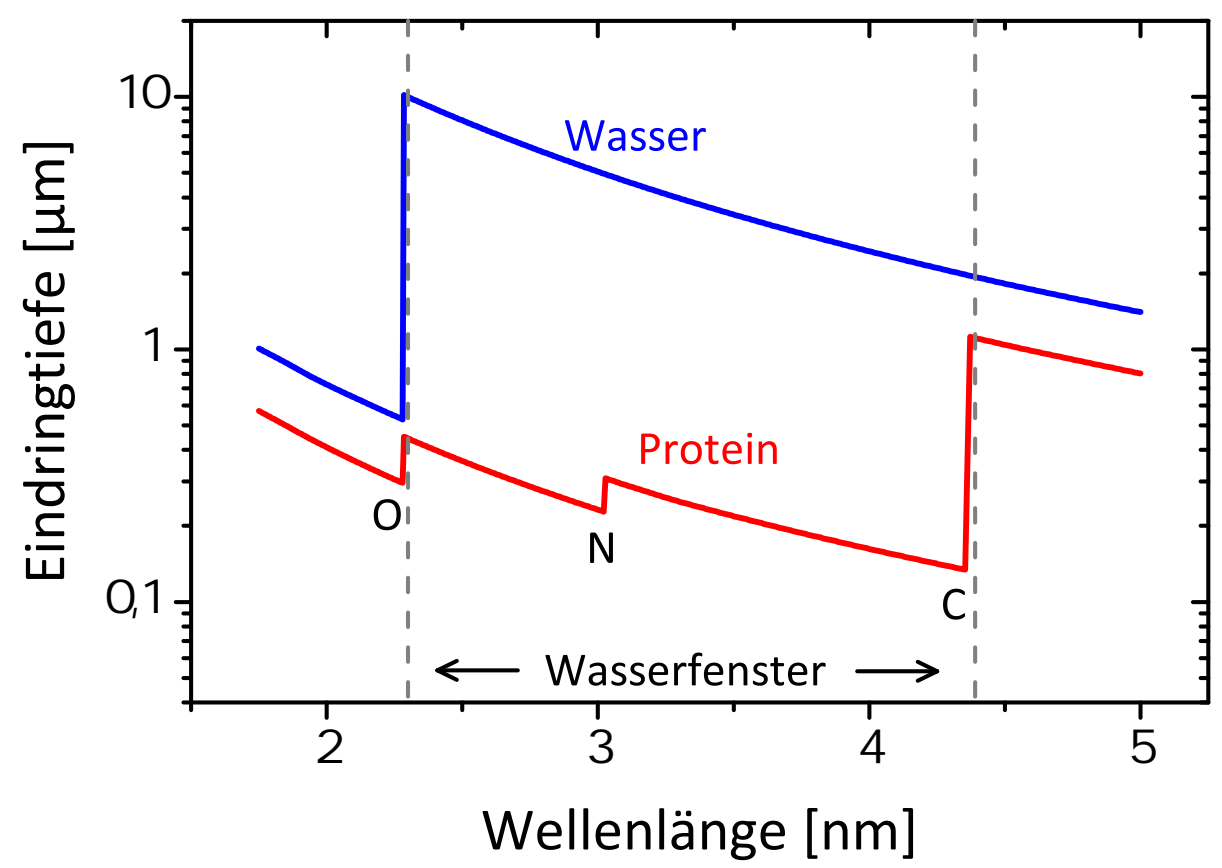

Abbildung 5.1: Eindringtiefen von Röntgenstrahlung in Wasser und einem typischen Protein $\left(\mathrm{C}_{94} \mathrm{H}_{134} \mathrm{~N}_{24} \mathrm{O}_{31} \mathrm{~S}\right.$ mit über 50\% Kohlenstoff-Anteil, nach [33]) in Abhängigkeit der Wellenlänge, welche das sog. „Wasserfenster“ definieren. Durch die C-Absorption und die hohe Durchstrahltiefe von Wasser lassen sich kontrastreiche röntgenmikroskopische Bilder biologischer Zellen erzeugen. (Die Absorptionsdaten sind der CXRO-Datenbank entnommen [40].)

keit aufwachsen sollen [92]. Bei der Herstellung reflektiver Multilagenoptiken wird in der Literatur häufig mit dem Materialsystem $\mathrm{Cr} / \mathrm{Sc}$ gearbeitet, welches sich aufgrund hoher Reflektivitäten im Wellenlängenbereich des Wasserfensters anbietet [158-160]. Im Rahmen dieser Arbeit sollen hingegen alternative Materialkombinationen Verwendung finden, die sich neben einem ausreichend hohen Unterschied im Absorptionskoeffizienten im Wasserfenster durch optimale Wachstumseigenschaften laserdeponierter Multischichten wie z. B. geringe Grenzflächenrauigkeiten auszeichnen [149,161]. In Abbildung 5.2 sind dazu die Absorptionslinien des zunächst benutzten Systems $\mathrm{Ti} / \mathrm{ZrO}_{2}$ und, zur besseren Übersicht bereits vorweg gegriffen, die des Systems $\mathrm{MgO} / \mathrm{ZrO}_{2}$ gezeigt, welches in Kapitel 5.2 zur Herstellung einfacherer Fokussierungsoptiken verwendet wurde. Beim Materialsystem $\mathrm{Ti} / \mathrm{ZrO}_{2}$ ergeben sich nur bei Wellenlängen oberhalb der Ti-Absorptionskante (wie auch bei der hier verwendeten mit $\lambda=2,879 \mathrm{~nm}$ ) große Unterschiede in $\beta$, sodass der zur Verfügung stehende Wellenlängenbereich in diesem System auf $\lambda=2,73-4,4 \mathrm{~nm}$ begrenzt ist. Beim System $\mathrm{MgO} / \mathrm{ZrO}_{2}$ stellt sich trotz der Verwendung zweier Oxide ebenfalls ein ausreichender Kontrast zur Herstellung 


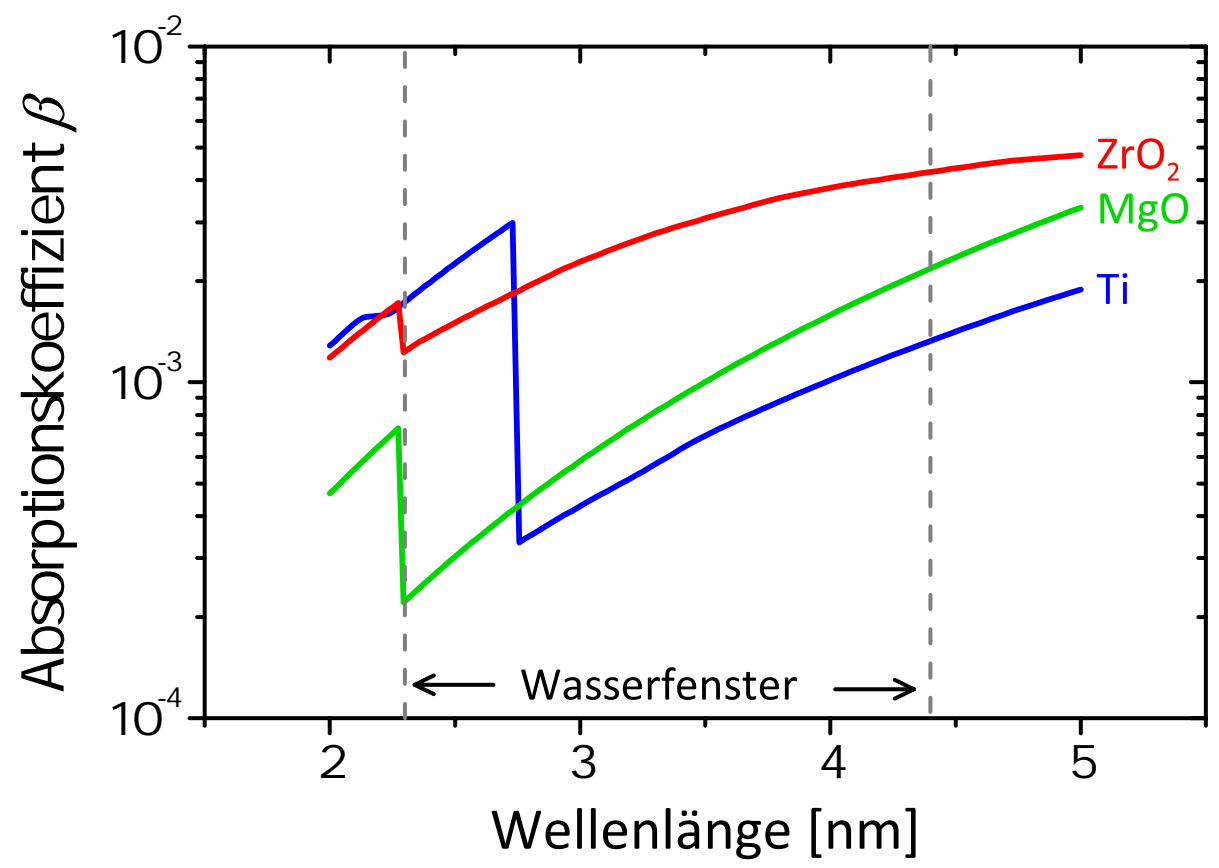

Abbildung 5.2: Absorptionskoeffizienten der zur Herstellung von MLL verwendeten Materialien $\mathrm{ZrO}_{2}$, $\mathrm{MgO}$ und Ti in Abhängigkeit von der Röntgenwellenlänge. Die Absorptionskante des Ti schränkt dabei die Verwendbarkeit innerhalb des Wasserfensters auf einen kleineren Wellenlängenbereich ab $\lambda=2,73 \mathrm{~nm}$ ein. (Die Absorptionsdaten sind der CXRO-Datenbank entnommen [40].)

effizienter Röntgenoptiken ein. In beiden Fällen ist $\mathrm{ZrO}_{2}$ das stärker absorbierende Material, sodass Ti bzw. MgO als Spacer eingesetzt werden.

Um mit der gegebenen Wellenlänge von $\lambda=2,879 \mathrm{~nm}$ die einzelnen Schichtdicken nach dem Zonenplattenbildungsgesetz berechnen zu können, muss noch die Fokuslänge festgesetzt werden, welche in Bezug auf die Geometrie des Mess-Aufbaus gewählt werden muss. Der Aufbau der „table-top“-Anlage wurde dazu wie in Kapitel 3.3 beschrieben variabel gestaltet, um die Fokuseigenschaften der MLL innerhalb der hier geplanten Experimente mit zwei unterschiedlichen Methoden zu untersuchen [157, 162]: Zum einen soll mittels einer Fernfelduntersuchung der propagierenden Röntgenstrahlung anhand der Erfassung von Intensitätsprofilen bei verschiedenen Detektorabständen rückwirkend auf die Fokuseigenschaften am Ort der Brennweite geschlossen werden. Zum anderen kann eine direkte Analyse nahe der Fokusebene durch sog. „knife-edge“Messungen durchgeführt werden, bei denen eine dünne absorbierende Kante senkrecht zur optischen Achse durch das Strahlprofil gefahren wird, um durch resultierende Intensitätsabfälle den Fokusdurchmesser zu bestimmen. Aufgrund dieser in $z$-Richtung durchzuführenden Messungen nahe der Fokusposition darf die Fokuslänge nicht zu kurz gewählt werden, obwohl so das Aufösungsvermögen nach Gleichung 2.21 gesteigert 
werden könnte. Für das erste auf diese Art und Weise geplante Design einer MLLTeststruktur wurde eine etwas größere Brennweite von $f=220 \mu \mathrm{m}$ angenommen.

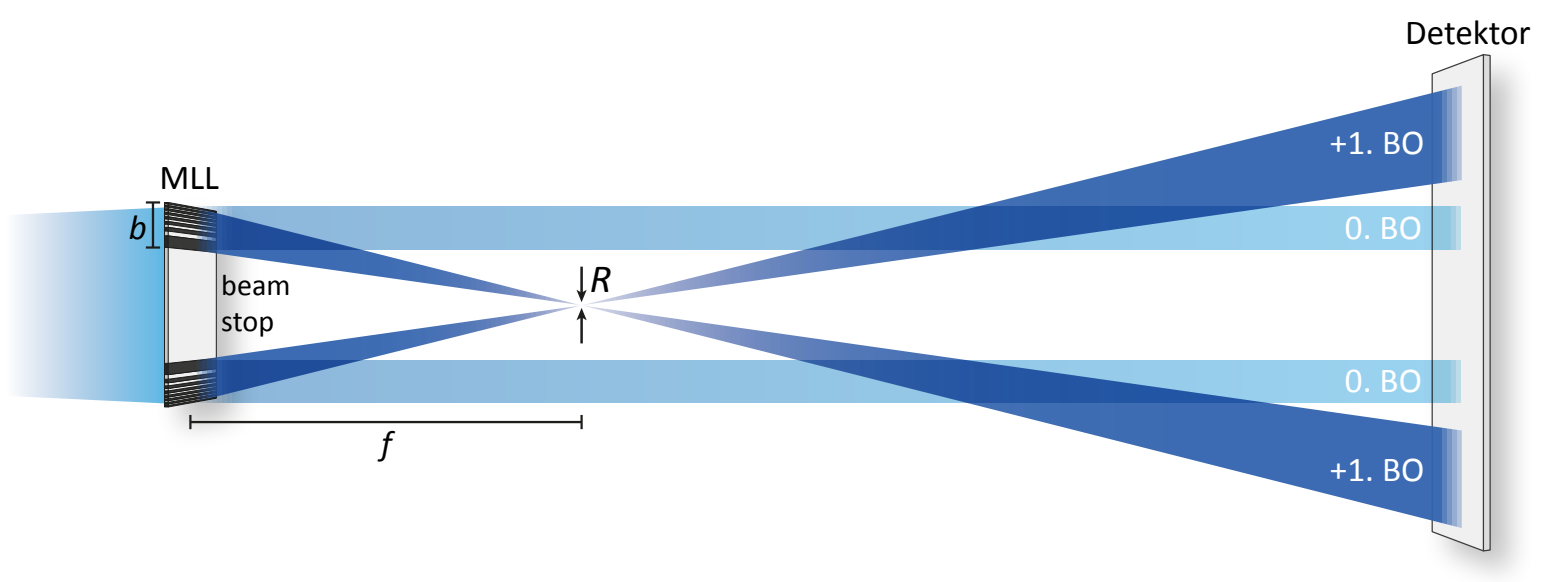

Abbildung 5.3: Schematische Darstellung der transmittierten Röntgenstrahlung durch die geplante MLL. Durch die Verwendung eines beam stops können im Fokus 1. Beugungsordnung Proben ohne Überlagerung der ungebeugten 0. Ordnung untersucht werden. (Zur Übersicht ist die MLL gegenüber dem Detektor stark vergrößert dargestellt.)

Abbildung 5.3 zeigt das Design der MLL-Teststruktur, welche für eine effiziente Fokussierung aus zwei aperiodischen Multischichtpaketen zusammengesetzt wurde. Dabei musste die eine Hälfte der Linse um $180^{\circ}$ gedreht werden, um die Röntgenstrahlung in einem Punkt konzentrieren zu können. Zwischen den beiden Teilen verhindert ein beam stop von ca. $11 \mu \mathrm{m}$ Größe, dass eine zu untersuchende Probe zusätzlich durch die 0. Beugungsordnung bestrahlt wird. Als Optik ergibt sich somit eine sog. off-axis-MLL, bei der die Deposition der dicksten Schichten ausgelassen wurde. Die Multischichtdeposition umfasste somit Schichtdicken zwischen der 49. Zone mit $d=57,1 \mathrm{~nm}$ und der 133. Zone mit $\Delta r=34,6 \mathrm{~nm}$. Eine Auswahl der einzelnen Zonenbreiten ist in Tabelle 5.1 zusammengetragen, mit denen die insgesamt 85 hergestellten Zonen eine Gesamtschichtdicke von $b=3,66 \mu \mathrm{m}$ einnehmen. Die optische Tiefe der Zonenplattenstruktur $\Delta t$ wurde mit $200 \mathrm{~nm}$ möglichst dünn festgelegt, um die Gesamtabsorption des MLL-Systems gering zu halten.

Bei der Abschätzung der zur erwarteten Fokusgröße muss unbedingt berücksichtigt werden, dass keine vollständige MLL fabriziert, sondern nur zwei Ausschnitte als off-axis-MLL zusammengefügt wurden. Für eine volle Zonenplattenstruktur würde sich mit oben angegebener äußerer Zonenbreite eine theoretische Auflösung von $R_{\text {ber. }}=1,22 \Delta r=42,2 \mathrm{~nm}$ ergeben. Bei der in diesem Fall hergestellten off-axis-MLL 


\begin{tabular}{llll}
\hline $\mathrm{n}$ & $d[\mathrm{~nm}]$ & $\mathrm{n}$ & $d[\mathrm{~nm}]$ \\
\hline & & & $\vdots$ \\
49 & 57,14 & & $\vdots$ \\
50 & 56,56 & 114 & 37,35 \\
51 & 55,99 & 115 & 37,19 \\
52 & 55,45 & 116 & 37,02 \\
53 & 54,92 & 117 & 36,87 \\
54 & 54,40 & 118 & 36,71 \\
55 & 53,90 & 119 & 36,55 \\
56 & 53,41 & 120 & 36,40 \\
57 & 52,94 & 121 & 36,25 \\
58 & 52,47 & 122 & 36,10 \\
59 & 52,02 & 123 & 35,95 \\
60 & 51,59 & 124 & 35,81 \\
61 & 51,16 & 125 & 35,66 \\
62 & 50,74 & 126 & 35,52 \\
63 & 50,33 & 127 & 35,38 \\
64 & 49,93 & 128 & 35,24 \\
65 & 49,55 & 129 & 35,10 \\
66 & 49,17 & 130 & 34,97 \\
67 & 48,79 & 131 & 34,83 \\
68 & 48,43 & 132 & 34,70 \\
69 & 48,08 & 133 & 34,57 \\
\hline
\end{tabular}

Tabelle 5.1: Nach dem binären Zonenplattenbildungsgesetz berechnete Einzelschichtdicken der geplanten MLL für $\lambda=2,879 \mathrm{~nm}$ und $f=220 \mu \mathrm{m}$. Mit steigender Anzahl an Zonen lässt sich aufgrund des wurzelförmigen Abfalls in der rechten Spalte ein geringerer Unterschied in den Schichtdicken mit einer annähernden Periodizität feststellen.

wird die Fokusgröße unabhängig von der kleinsten Zonenbreite anhand Gleichung 2.28 $\mathrm{zu}$

$$
R_{\text {ber. }}=0,61 \frac{\lambda f}{b}=105,4 n m
$$

berechnet. Abbildung 5.4 verdeutlicht diesbezüglich die theoretische Auflösung als Funktion der Aperturgröße der MLL. Da insgesamt nur eine 3,66 $\mu \mathrm{m}$ große Multischicht hergestellt wurde, ergibt sich bei gleicher äußerer Zonenbreite eine niedrigere Auflösung von etwa $105 \mathrm{~nm}$. Wird weiterhin die reale endliche Quellgröße des laserproduzierten Röntgenplasmas berücksichtigt, ist mit einer noch geringeren Auflösung im Laborexperiment zu rechnen. 


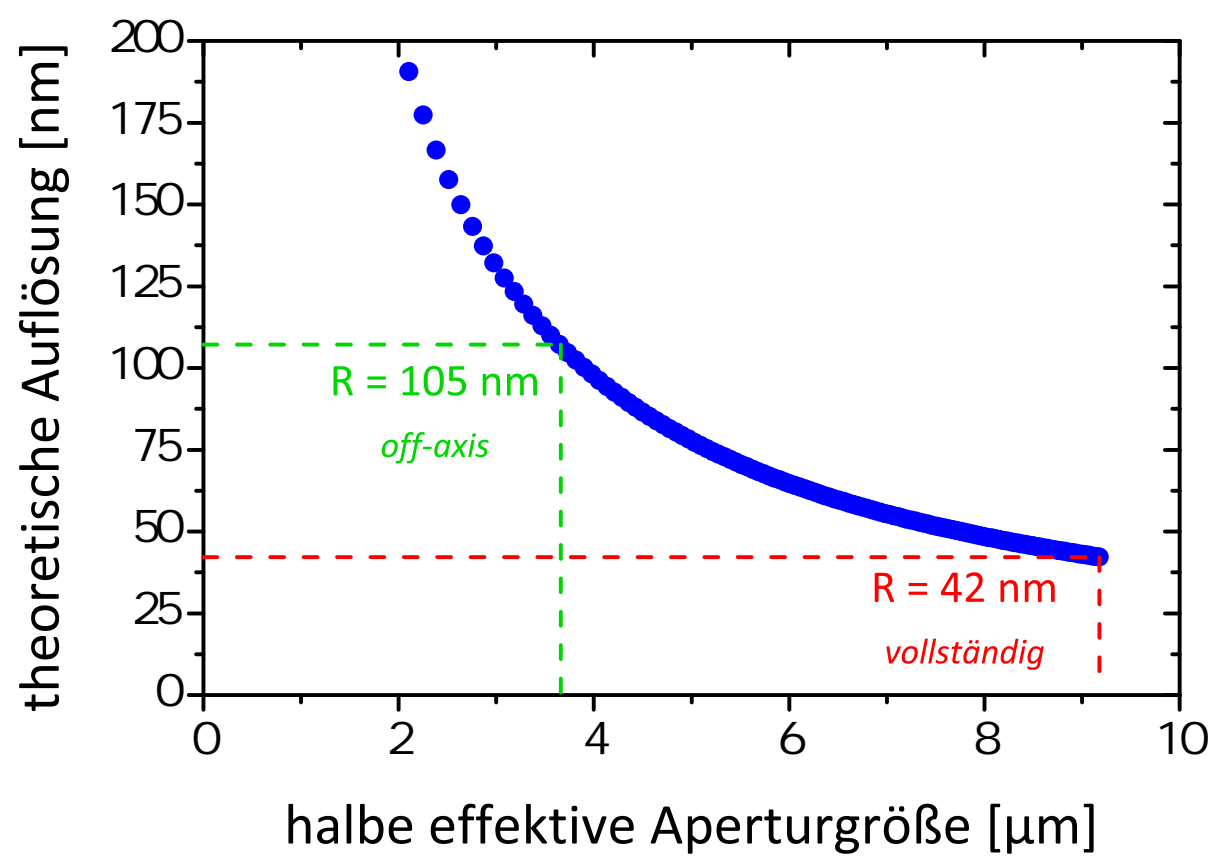

Abbildung 5.4: Theoretische Auflösung einer MLL mit $f=220 \mu \mathrm{m}$ und $\lambda=2,879 \mathrm{~nm}$ in Abhängigkeit der Aperturgröße. Trotz gleicher äußerer Zonenbreite ergibt sich für eine off-axis-MLL, bei der dickere Zonen nahe der optischen Achse ausgelassen wurden, eine niedrigere Auflösung.

\subsubsection{Charakteristik von laserdeponiertem $\mathrm{Ti}$ und $\mathrm{ZrO}_{2}$}

Bevor mit der Fabrikation einer $\mathrm{Ti} / \mathrm{ZrO}_{2}-\mathrm{MLL}$ begonnen werden kann, mussten zunächst die Wachstumseigenschaften des Materialsystems untersucht werden, um optimale Parameter zur Herstellung qualitativ hochwertiger Schichtpakete mittels der gepulsten Laserdeposition zu finden. Dazu wurden die beiden Materialien anfangs separat untersucht und später als periodisches Multischichtsystem zur Vorbereitung auf die Herstellung von MLL betrachtet. Da bei der geplanten MLL-Gesamtschichtdicke von 3,66 $\mu \mathrm{m}$ die Hälfte der Multischicht aus Ti besteht, die ermittelte Depositionsrate allerdings ungefähr 10 mal kleiner ist als die des $\mathrm{ZrO}_{2}$, sind auch ungefähr 10 mal so viel Laserpulse nötig, um die gewünschte Schichtdicke zu erreichen. Damit einhergehend findet die für die Laserdeposition typische Entstehung von Tröpfchen (Droplets) hauptsächlich am Ti-Target statt. Somit ist die Wahl geeigneter Herstellungsparameter für Ti deutlich kritischer als für $\mathrm{ZrO}_{2}$. Im ersten Schritt wurde daher eine Maximierung der Depositionsrate von Ti bei gleichzeitiger Tröpfchenminimierung vorgenommen.

Wie in Abbildung 5.5 gezeigt, wurden diesbezüglich die Depositionsrate, gemessen mit dem in Kapitel 3.5 vorgestellten Ratenmonitors, sowie die Dropletdichten bei variabler Energiedichte $\rho=E / A$ des Lasers mittels einer Verschiebung der Fokus- 
sierungslinse analysiert, indem bei gleichbleibender Laserenergie $E$ die Brennfleckgröße des Laserstrahls $A$ verändert wurde. Die Linsenposition entspricht dabei dem Abstand $d$ des Linsenfokus zur Targetoberfläche. Wird zunächst die Depositionsrate im Bereich $d=0-7 \mathrm{~cm}$ betrachtet, so ergibt sich durch lineare Anpassung der Datenpunkte bis zum Maximum der Rate die Depositionsschwelle bei knapp $8 \mathrm{~cm}$, bei der die eingestrahlte Energiedichte nicht mehr ausreicht, um Targetmaterial abzutragen. Das Maximum mit $0,0042 \mathrm{~nm} /$ Puls hingegen befindet sich nicht bei fokussierter Geometrie $(0 \mathrm{~cm})$, sondern bereits bei 2,6 cm defokussierter Linsenstellung. Durch weitere Fokussierung kann die Depositionsrate aufgrund des sog. „,shielding“-Effekts [163] nicht mehr erhöht werden, da die oberhalb der Targetoberfläche befindliche gasförmige Knudsenlage bereits vollständig gesättigt ist und einen Großteil des Laserpulses absorbiert. Vielmehr fällt die Depositionsrate durch eine Erhöhung der Brennfleckgröße $(d<0)$ bei resultierender kleinerer Energiedichte wieder ab.

Im unteren Graph der Abbildung 5.5 sind die Dropletdichten bei verschiedenen Linsenpositionen aufgetragen, die anhand von hergestellten Ti-Schichten ausgewertet und auf $100 \mathrm{~nm}$ Einzelschichtdicke bei einer Oberfläche von $10000 \mu \mathrm{m}^{2}$ normiert wurden. Dabei werden die Droplets bezüglich ihrer Herkunft am Target in große $(>0,3 \mu \mathrm{m})$ und kleine Droplets $(<0,1 \mu \mathrm{m})$ klassifiziert [135]. Typisch für die Laserablation von Metallen bilden sich nach FäHLER et al. große Droplets hauptsächlich durch eine dem einfallenden Laserstrahl ausgerichtete Zapfenbildung am Target (vgl. Abb. 5.6), wobei die Droplets entweder an den Spitzen der Zapfen durch ständiges Aufschmelzen und Abkühlen von Targetmaterial entstehen oder aber die säulenartigen Zapfen anhand von thermischen Schockwellen komplett abgerissen werden und sich als flüssige Tröpfchen auf dem Substrat niederschlagen. Kleine Droplets entstehen durch das Vorhandensein leichter Unebenheiten der Targetoberfläche, welche aufgrund der ständigen Schmelz- und Erstarrungsvorgängen wachsen und schließlich abgetragen werden. Dieser PLD-Prozess wird in der Literatur als „hydrodynamisches Sputtern“ bezeichnet [164]. In Abbildung 5.5 unten zeigt sich ein Minimum der kleinen Droplets ungefähr bei derjenigen Linsenposition, bei der sich das Maximum der Depositionsrate einstellt. Dort werden insgesamt weniger Laserpulse zur angestrebten Schichtdicke benötigt, sodass die resultierende Dropletanzahl am geringsten ist. Mit steigender Defokussierung wird sodann die eingekoppelte Energiedichte pro Laserpuls verringert, sodass ein Flächenelement des Targets wesentlich häufiger bestrahlt werden muss, um die gleiche Schichtdicke wie im Maximum zu erzielen. Das damit einhergehende vermehrte Aufschmelzen und Abkühlen führt wiederum zu einer Erhöhung der Dichte 


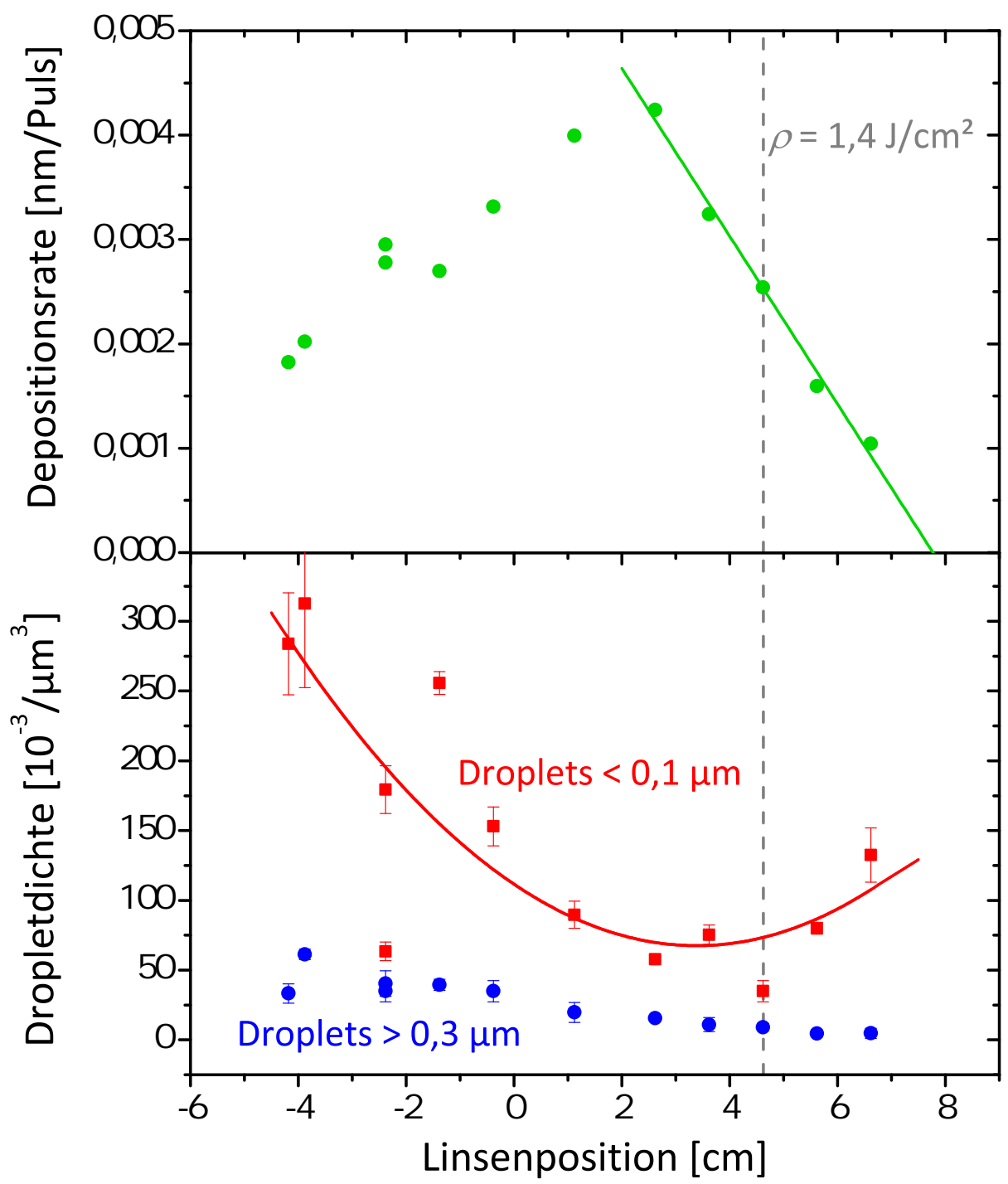

Abbildung 5.5: Depositionsrate sowie auf $100 \mathrm{~nm}$ Schichtdicke normierte Dropletverteilung von Ti in Abhängigkeit der Fokussierung des einfallenden Laserstrahls. Bei steigender Rate (v. r. n. l.) und somit für eine gleiche Schichtdicke weniger benötigte Laserpulse existieren weniger kleine Droplets, größere werden mit steigender Fokussierung vermehrt gezählt. Die Klassifizierung großer und kleiner Droplets wurde nach ihrer Herkunft am Target vorgenommen [135]. Die Linsenposition für zukünftige Schichtherstellung wurde bezüglich niedrigster Gesamtanzahl an Droplets gewählt.

kleinerer Droplets. Große Droplets wurden im Bereich fokussierter Linsenstellung d. h. bei maximaler Energiedichte am häufigsten gezählt. Mit abnehmender Energiedichte scheinen weniger Zapfen abgerissen zu werden, sodass die Dropletanzahl wieder sinkt. Zusätzlich ist im Graph diejenige defokussierte Linsenstellung bei 4,6 cm eingezeichnet, welche für zukünftige Experimente als Standard-Parameter definiert wurde. Dieser entspricht der Linsenposition, bei der insgesamt am wenigsten Ti-Droplets vorhanden 
sind. Mit einer festgelegten Laserenergie von $E=70 \mathrm{~mJ}$ ergibt sich bei einer bestrahlten Targetfläche von ca. $A=5 \mathrm{~mm}^{2}$ eine Energiedichte von $\rho=E / A=1,4 \mathrm{~J} / \mathrm{cm}^{2}$. Die zugehörige Depositionsrate beträgt $0,0025 \mathrm{~nm} / \mathrm{Puls}$.
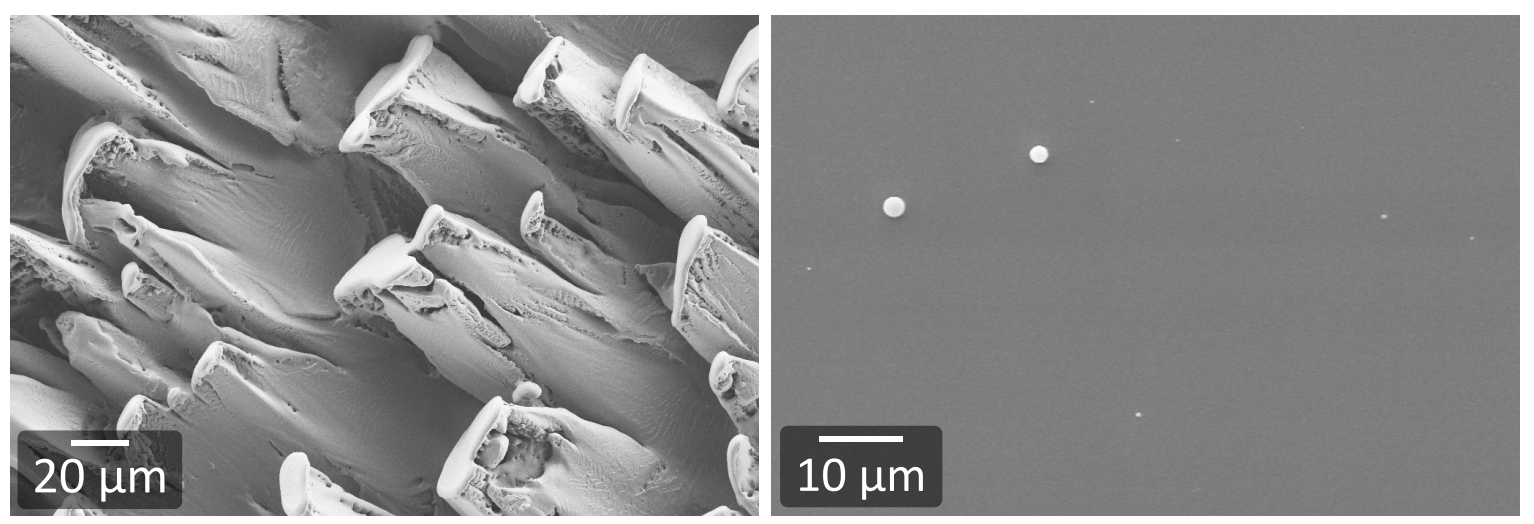

Abbildung 5.6: REM-Aufnahmen eines Ti-Targets (links) und einer damit hergestellten $75 \mathrm{~nm}$ dicken Einzelschicht (rechts) bei optimaler Linsenposition. In der Zapfenstruktur des Targets ist im Wesentlichen der Ursprung großer Droplets zu sehen, welche allerdings nur in geringem Maße auf der Schichtoberfläche vorhanden sind.

Die bei optimierter Wahl der Linsenposition bestrahlte Targetoberfläche von Ti ist in Abbildung 5.6 links dargestellt, bei der die dem Laserstrahl entgegengerichteten Zapfen erkennbar sind. Diese weisen allerdings keine vollständige, säulenartige Struktur auf, was auf ein Abreißen des vorderen Zapfenteils durch die intensive Laserbestrahlung und folglich auf eine Produktion einiger großen Droplets hindeutet. Im rechten Teil der Abbildung 5.6 ist eine REM-Aufnahme einer $75 \mathrm{~nm}$ dicken Ti-Einzelschicht gezeigt, welche aufgrund der geeigneten Wahl der Linsenposition sehr wenige große und kleine Droplets aufweist. Eine Untersuchung der Wachstumsorientierung einer TiEinzelschicht auf einem Si(111)-Substrat wurde anhand einer RöntgendiffraktometrieMessung vollzogen. Das zugehörige Spektrum im Winkelbereich zwischen $35^{\circ}$ und $100^{\circ}$ in $2 \theta$ ist in Abbildung 5.7 dargestellt. Demnach wächst Ti mit ca. 93\% der Gesamtintensität bevorzugt in der $<100>-$ und $\mathrm{zu}$ einem geringen Anteil in $<001>$-Richtung auf. Die mittlere Korngröße wurde anhand der Scherrer-Formel (vgl. Kap 3.6.2) aus der Halbwertsbreite der Reflexe zu 14 nm abgeschätzt.

Nachdem für Ti optimale Herstellungsbedingungen ermittelt wurden, soll im Folgenden die Laserdeposition von $\mathrm{ZrO}_{2}$ untersucht werden. Da die Position der Fokussierungslinse während einer Multischichtherstellung nicht verändert werden konnte, wurde für diese Komponente die gleiche Linsenposition von $4,6 \mathrm{~cm}$ und somit die gleiche Energiedichte von $\rho=1,38 \mathrm{~J} / \mathrm{cm}^{2}$ gewählt. Als Targetmaterial fand polykristallines $\mathrm{ZrO}_{2}$ Verwendung, welches mit $3 \mathrm{~mol} \% \mathrm{Y}_{2} \mathrm{O}_{3}$ in dessen tetragonaler Kristallform partiell 


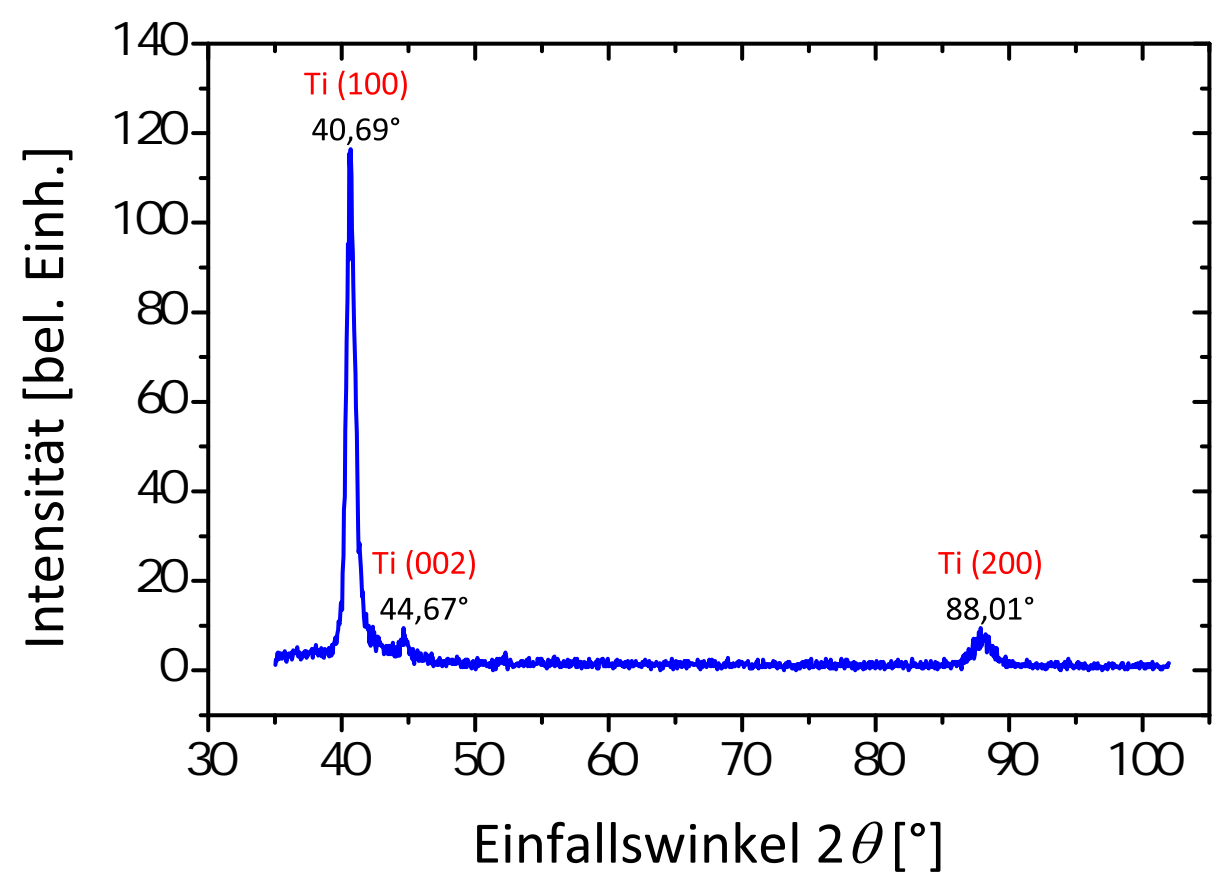

Abbildung 5.7: Röntgendiffraktogramm einer laserdeponierten Ti-Schicht. Das kristalline Wachstum mit einer durchschnittlichen Korngröße von $14 \mathrm{~nm}$ zeigt eine Vorzugsorientierung in <100>Richtung mit ungefähr $93 \%$ der gestreuten Intensität auf.

stabilisiert ist. Anhand der mikroskopisch untersuchten Targetoberfläche (s. Abb. 5.8 oben) zeigt sich in der Übersicht eine sehr glatte Morphologie des $\mathrm{ZrO}_{2}$-Targets, welche für einen gleichmäßigen Materialabtrag mit einer geringen Anzahl an Droplets spricht. Allerdings entstehen mit zunehmender Laserpulszahl immer mehr und tiefere Spannungsrisse entlang der Korngrenzen, wie es in den Detailaufnahmen der Abbildung 5.8 unten gezeigt ist. Die Entstehung solcher Spannungsrisse ist in einem ständigen Wechsel von Aufschmelzen und Abkühlen während der Ablation des Targetmaterials zu sehen. Mit zunehmender Anzahl der Schmelz- und Erstarrungsvorgänge reichen einige Risse tiefer in das Material hinein, wodurch lokale Spannungsfelder kompensiert werden (s. Abb. 5.8 rechts). Im Energiedichtebereich zwischen 1,2 und 1,5 J/ $\mathrm{cm}^{2}$, in dem die hier gewählte Energiedichte liegt, erfolgt während der Ablation eine laserinduzierte partielle Umwandlung von der tetragonalen zur (bei Raumtemperatur) stabilen monoklinen Kristallphase, die für eine hohe Rissfestigkeit des Targetmaterials sorgt [165]. Ohne eine entsprechende Dotierung und Festlegung der Energiedichte würde weitere Bestrahlung eine Delamination von Targetmaterial („exfoliational sputtering“ [166]) zur Folge haben, sodass die Herstellung partikelfreier Filme (mit Ausnahme herkömmlicher Droplets) nicht mehr gewährleistet wäre. 

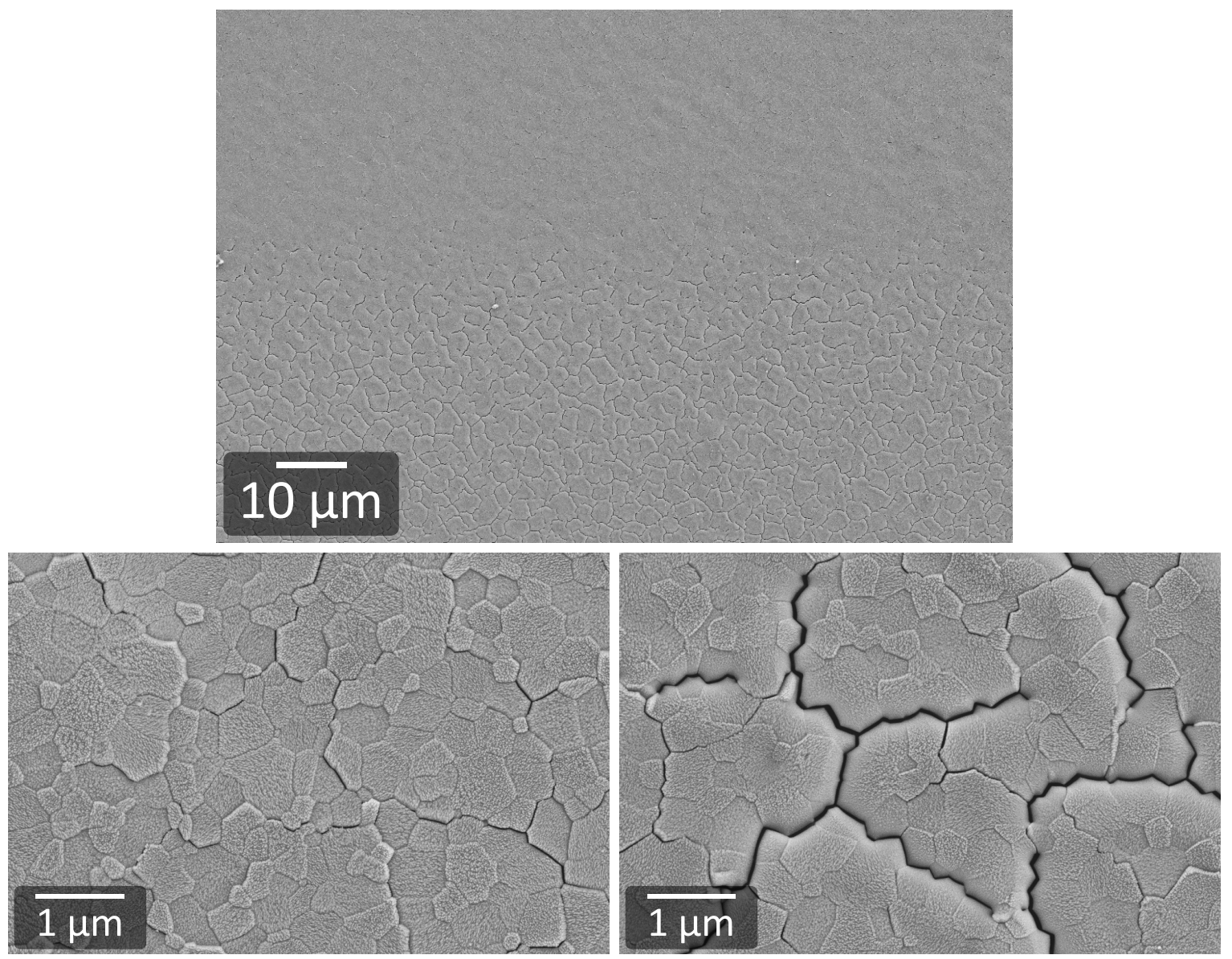

Abbildung 5.8: REM-Aufnahmen des verwendeten $\mathrm{ZrO}_{2}$-Targets, welches mit $3 \mathrm{~mol} \% \mathrm{Y}_{2} \mathrm{O}_{3}$ partiell stabilisiert ist. Die sehr glatte Oberfläche (oben) ist je nach Häufigkeit lokaler Laserbestrahlung unterschiedlich stark strukturiert: Der etwas weniger abgetragene Bereich zeigt in der Detailaufnahme links Spannungsrisse entlang der Korngrenzen, durch die lokale Spannungsfelder ausgeglichen werden. Mit zunehmender Bestrahlung nehmen auch die Spannungen zu, sodass in der rechten Detailansicht einige Risse wesentlich tiefer in das Targetmaterial hineinreichen.

Somit lässt sich bei der Analyse einer $3,2 \mu \mathrm{m}$ dicken periodischen $\mathrm{Ti} / \mathrm{ZrO}_{2}-$ Multischicht in Abbildung 5.9 hauptsächlich die Existenz von Ti-Droplets bestätigen. Neben runden Tröpfchen sind unter flachem Betrachtungswinkel auch kegelförmige erkennbar, die schon relativ früh während der Deposition entstanden sein müssen, da sie im weiteren Herstellungsprozess mitbeschichtet wurden. Insgesamt ist die Dropletdichte so gering, dass für die Herstellung einer MLL stets eine makellose Substratposition gefunden werden kann.

Zur Überprüfung eines exakten Stöchiometrie-Übertrags des $\mathrm{ZrO}_{2}$ zwischen Target und Substrat soll der Ablationsprozess genauer untersucht werden. Nach Voevodin et al. befinden sich im Laserplasma die Atome bzw. Moleküle Zr, O, 


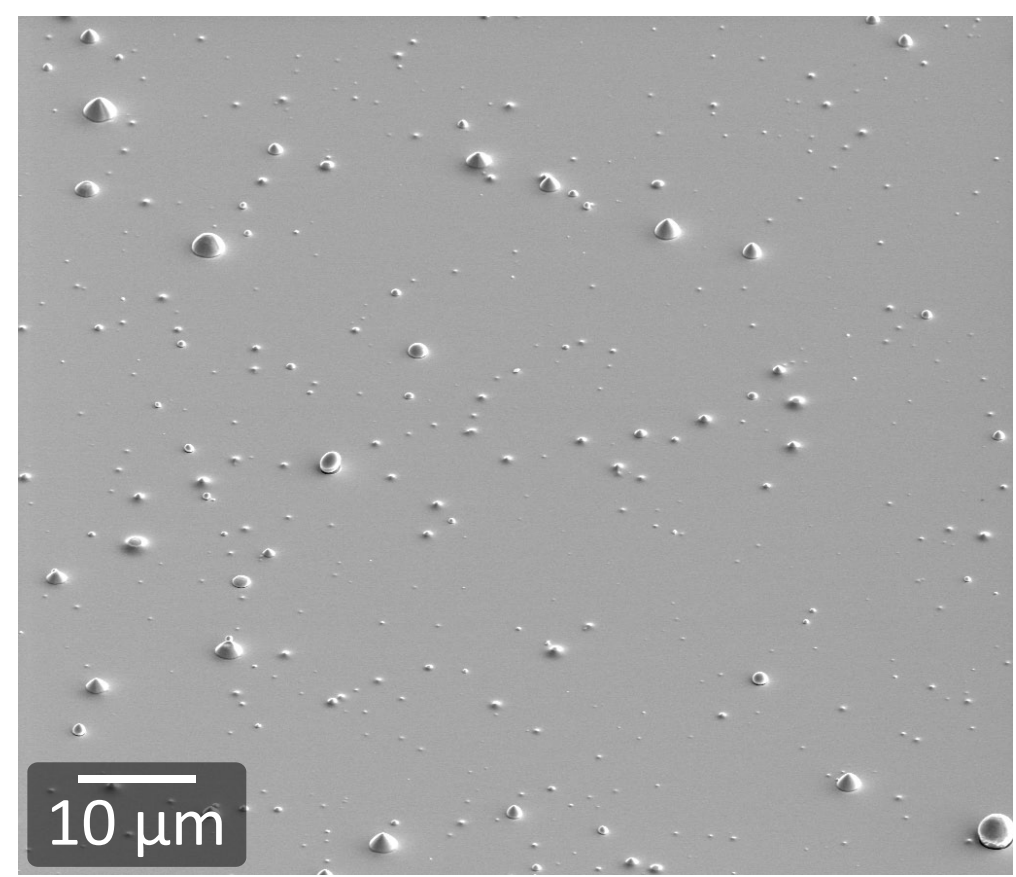

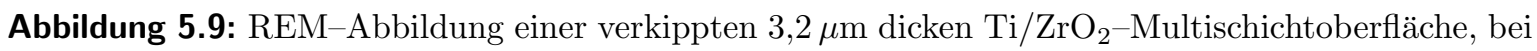
der sich zwischen den Droplets geeignete Positionen zur MLL-Herstellung finden lassen.

$\mathrm{ZrO}, \mathrm{O}_{2}$ sowie $\mathrm{Y}$ und $\mathrm{YO}$ im angeregten Zustand, sodass $\mathrm{ZrO}_{2}$ nicht als molekulare Einheit zum Substrat übertragen wird [167]. Ob sich die Rekombination der Atome im Plasma bzw. auf der Substratoberfläche stöchiometrisch vollzieht, wurde mit Hilfe einer EUV-Reflektometrie-Messung (s. Kap. 3.6.3) an einer $1 \mu$ m dicken $\mathrm{ZrO}_{2}{ }^{-}$ Einzelschicht überprüft. Bei einer solch großen Schichtdicke treten keine störenden Grenzflächenoszillationen zwischen Schicht und Substrat auf, sodass das Spektrum dieser bulk-Probe einfacher zu simulieren ist.

Die Messung und zugehörige Simulation wurden von Stefan Döring im LaserLaboratorium Göttingen e. V. mittels eines EUV-Reflektometers $\left(\lambda=12,98 \mathrm{~nm}, \mathrm{O}_{2}-\right.$ Absorptionskante) durchgeführt. Reflektometrie-Messungen bei großen Wellenlängen haben prinzipiell den Vorteil, dass die resultierenden Spektren über einen großen Winkelbereich aufgezogen werden und somit im Vergleich zur konventionellen Röntgenreflektometrie mit harter Röntgenstrahlung eine bessere Auflösung bieten. Wie in Abbildung $5.10 \mathrm{zu}$ sehen, erstreckt sich die Totalreflexionskante der $\mathrm{ZrO}_{2}$-Schicht über einen breiten Winkelbereich bis $\theta=20^{\circ}$, sodass mittels ihrer Simulation genaue Informationen über die partiellen Dichten der Komponenten und somit über die chemische Zusammensetzung gewonnen werden können. Im Ergebnis zeigt sich hierbei ein Stöchiometrie-Verhältnis von 1:1,8 zwischen $\mathrm{Zr}$ und $\mathrm{O}$, welches für ein leichtes 


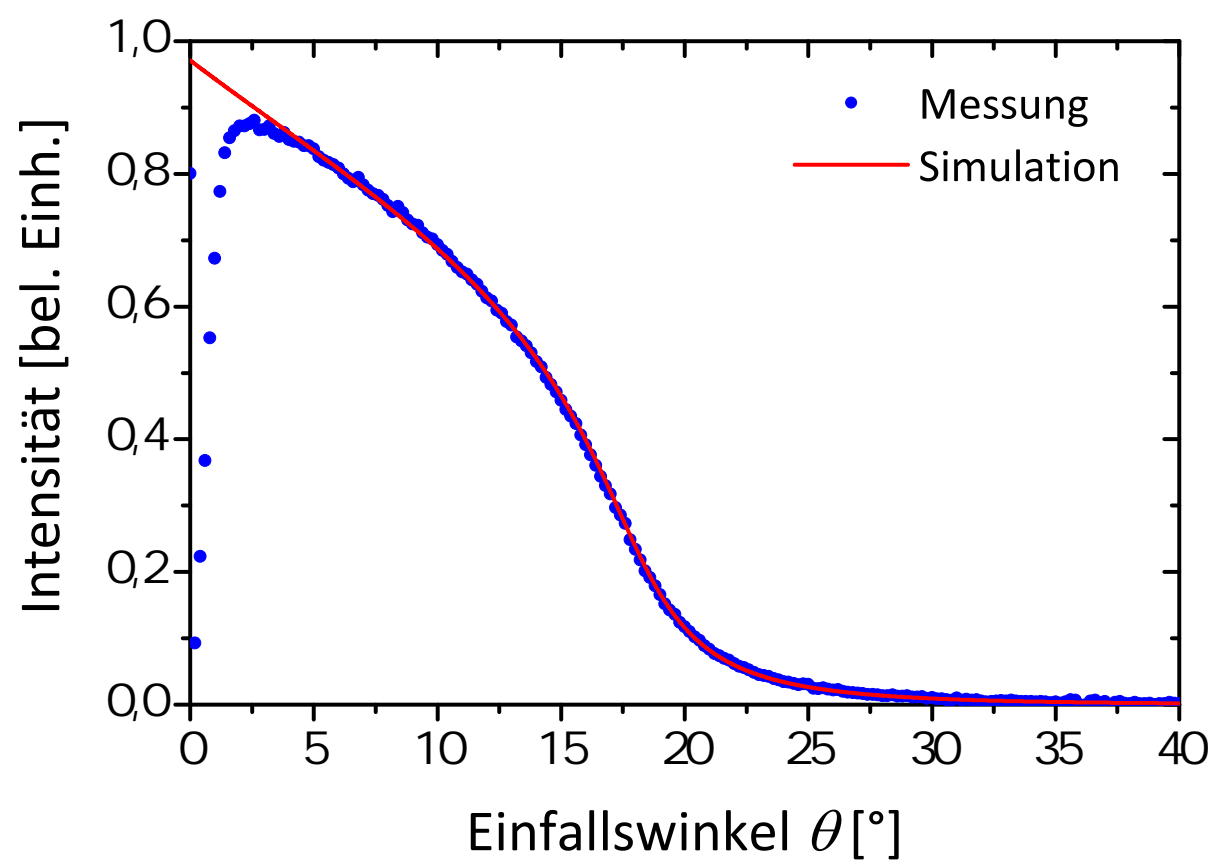

Abbildung 5.10: Reflektometrie mit extrem ultravioletter Strahlung an laserdeponiertem $\mathrm{ZrO}_{2}$. Die Simulation des hochaufösenden Spektrums offenbart ein leichtes Sauerstoffdefizit von $\mathrm{ZrO}_{1,8}$ aufgrund eines nicht exakt stöchiometrischen Materialübertrags von Target zu Substrat. (Die Daten wurden freundlicherweise von Stefan Döring zur Verfügung gestellt.)

Sauerstoffdefizit der laserdeponierten Schicht spricht. Diese nicht vollständige Rekombination von $\mathrm{Zr}$ und $\mathrm{O}$ wird von HEIROTH et al. durch eine unzureichende Haftung oder ein präferentielles Absputtern der leichten O-Atome bzw. -Moleküle erklärt [168]. In ihren Experimenten wurde unter Vakuumbedingungen ein Sauerstoffdefizit von $\mathrm{ZrO}_{1,77}$ gemessen, welches mit den hier erhaltenen Ergebnissen in guter Übereinstimmung steht. Für die Herstellung einer MLL hat der Verlust von Sauerstoff allerdings keine nachteiligen Konsequenzen. Da $\mathrm{ZrO}_{2}$ im Ti/ZrO${ }_{2}$-System als Absorber fungiert, würde ein O-Defizit dessen Absorption zusätzlich erhöhen, sodass die optischen Eigenschaften sogar leicht verbessert würden. Darüber hinaus kann es im $\mathrm{Ti} / \mathrm{ZrO}_{2}-$ Schichtverbund sogar zu einem noch höheren als dem hier gemessenen Defizit kommen, zumal Ti ein reaktiver O-Getter ist und an den Grenzflächen eine bevorzugte Bildung von $\mathrm{TiO}_{2}$ bewirken kann. Dieser Effekt würde wiederum zu einem vergrößerten Unterschied der Absorptionskoeffizienten von $\mathrm{Ti}$ und $\mathrm{ZrO}_{2}$ führen, was im Rahmen dieser Arbeit allerdings nicht eingehender untersucht wurde. Im Vergleich von laserdeponierten und gesputterten $\mathrm{ZrO}_{2}$-Schichten besitzen die PLD-hergestellten nicht nur eine genauere chemische Zusammensetzung, sondern zeigen auch eine geringere Rauigkeit als die gesputterten Schichten [169]. 


\subsubsection{Untersuchungen am $\mathrm{Ti} / \mathrm{ZrO}_{2}-$ Multischichtsystem}

Nachdem die Ablationseigenschaften der einzelnen Komponenten bei den optimalen Herstellungsparametern untersucht wurden, sollen nun die Wachstumseigenschaften von $\mathrm{Ti} / \mathrm{ZrO}_{2}-\mathrm{Multischichten}$ betrachtet werden. Insbesondere ist dabei die Beschaffenheit der Grenzflächen bezüglich chemischer Stabilität und geringer Rauigkeit von Interesse. Bei dem im Rahmen dieser Arbeit vorgestellten $\mathrm{Ti} / \mathrm{ZrO}_{2}-$ Schichtsystem ist der glättende Charakter des $\mathrm{ZrO}_{2}$ äußerst hilfreich, der Bildung einer kumulativen Rauigkeit vorzubeugen. Nach den Untersuchungen von RöDER et al. zeigt laserdeponiertes $\mathrm{ZrO}_{2}$ nicht nur eine sehr niedrige intrinsische Rauigkeit von $0,1 \mathrm{~nm}$, sondern bewirkt zusätzlich ein frequenzabhängiges Ausglätten vorhandener Rauigkeiten in Abhängigkeit der deponierten Schichtdicke [170]. Hohe Frequenzen, also Rauigkeiten mit kleinen Wellenlängen werden demnach mit steigender $\mathrm{ZrO}_{2}$-Schichtdicke zuerst ausgeglättet, während bei langwelligeren größeren Strukturen Schichtdicken über $1 \mu \mathrm{m}$ für eine effiziente Glättung benötigt werden. Diesem zweistufigen Prozess konnten hierbei unterschiedliche Glättungsmechanismen zugeordnet werden [171]: Kleine Oberflächenstrukturen bis zu einer Größe von ungefähr $70 \mathrm{~nm}$ werden durch Diffusionsprozesse geglättet, welche zudem durch die hohen kinetischen Energien der deponierten Ionen während der gepulsten Laserdeposition begünstigt werden. Größere Strukturen werden entweder durch Sputtererosion oder ballistischen Transport ausgeglättet. Bei der Sputtererosion findet ein Wiederabtrag von Schichtmaterial statt, der sowohl einen glättenden als auch aufrauenden Einfluss auf die deponierte Schicht nehmen kann. Ballistischer Transport wird durch Stoßkaskaden senkrecht zu einer lokalen Oberflächennormale beschrieben, bei der auf einer Erhebung befindliche Atome lawinenartig in ein benachbartes Tal geschoben werden.

$\mathrm{Ob}$ die Sputtererosion bei laserdeponierten $\mathrm{Ti} / \mathrm{ZrO}_{2}-$ Multischichten existiert und aufgrund eines möglichen Wiederabtrags der aufgewachsenen Ti-Schicht einen Einfluss auf die einzuhaltenden Schichtdicken nimmt, wurde anhand einer Depositionsratenmessung von $\mathrm{Ti}$ und $\mathrm{ZrO}_{2}$ in Abhängigkeit der abgegebenen Laserpulse untersucht [149]. In Abbildung 5.11 ist diesbezüglich die auf einen Ratenmonitor deponierte $\mathrm{Ti}-$ und $\mathrm{ZrO}_{2}-$ Schichtdicke über mehrere Grenzflächen aufgeführt. Würde sich ein merklicher Abtrag von Schichtmaterial und somit ein Sputteryield an einer Grenzfläche einstellen, wäre dies durch eine lokale Abnahme der Schichtdicke unmittelbar hinter einem Grenzflächenübergang erkennbar. Bei der hier gezeigten Messung verlaufen jedoch sämtliche Dickenzunahmen mit steigender Anzahl an Laserpulsen linear, was für eine Konstanz der Depositionsraten der einzelnen Materialien spricht. In diesem 
Fall belaufen sie sich auf $0,1 \mathrm{~nm} / \mathrm{Puls}$ für $\mathrm{ZrO}_{2}$ und $0,03 \mathrm{~nm} / \mathrm{Puls}$ für Ti. Beim Wachstum von $\mathrm{ZrO}_{2}$ auf $\mathrm{Ti}$ und umgekehrt ist demnach nicht von einer Existenz eines Wiederabsputtereffekts auszugehen (vgl. dazu die Grenzflächenmessung am W/SiSystem in Abbildung 6.12 ff., bei der dieser Effekt deutlich erkennbar ist). Für die Glättung durch $\mathrm{ZrO}_{2}$ bedeutet dies, dass beim laserdeponierten $\mathrm{Ti} / \mathrm{ZrO}_{2}-$ System Oberflächendiffusion sowie ballistischer Transport die dominierenden Mechanismen darstellen.

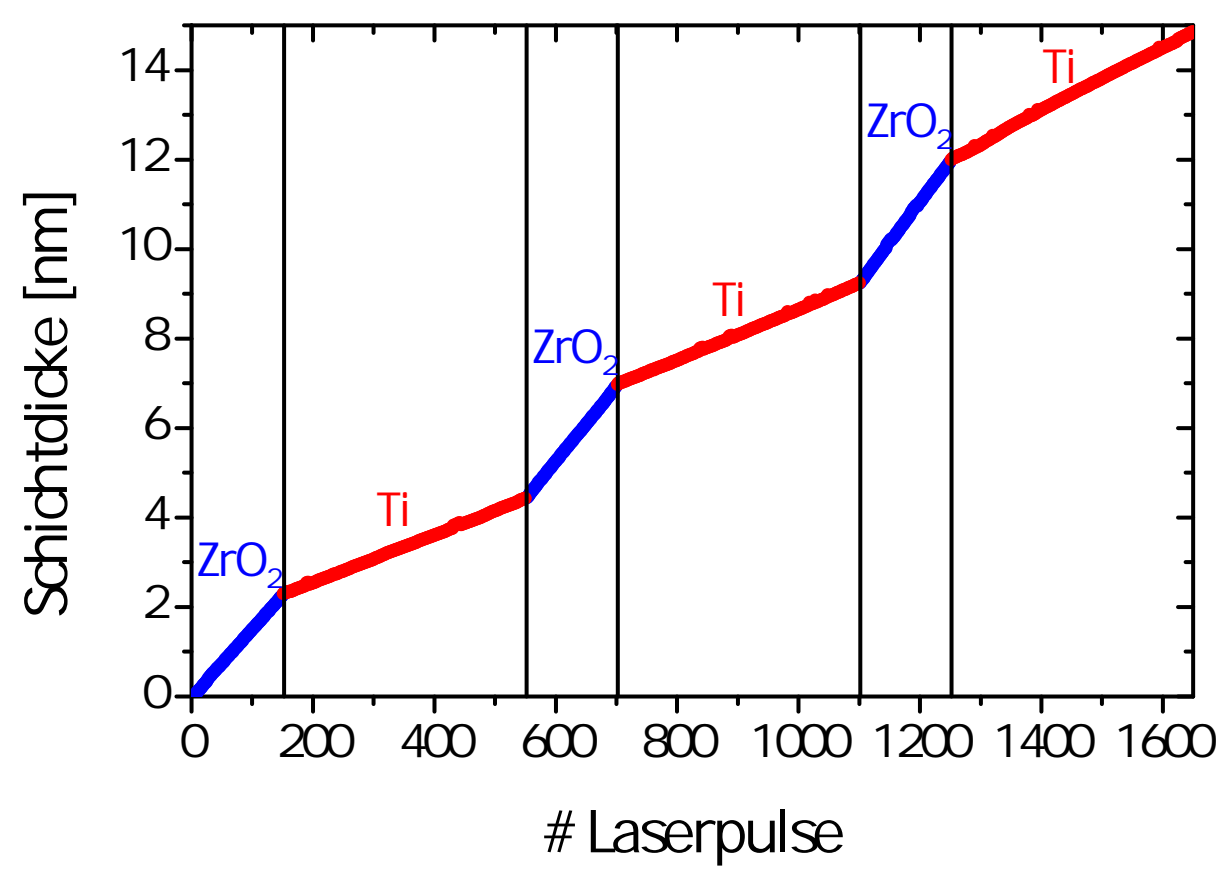

Abbildung 5.11: Schichtdickenverlauf eines $\mathrm{Ti} / \mathrm{ZrO}_{2}-$ Multischichtsystems über mehrere Grenzflächen in Abhängigkeit der Laserpulszahl. Unterschiedliche Steigungen entsprechen hier unterschiedlichen Depositionsraten von $\mathrm{ZrO}_{2}$ und Ti. An den Grenzflächen sind aufgrund konstanter Steigungen keine Absputtereffekte erkennbar (aus [149]).

Die Auswirkungen einer effektiven Glättung zeigen sich anhand von REMAufnahmen einer $\mathrm{Ti} / \mathrm{ZrO}_{2}-$ Multischicht mit 140 Doppelschichten (s. Abb. 5.12, Ti dunkel, $\mathrm{ZrO}_{2}$ hell), bei denen sich keinerlei Hinweise auf eine Entstehung kumulativer Rauigkeit andeuten [171]. Vielmehr lässt sich hier sogar die sukzessive Glättung eines $100 \mathrm{~nm}$ breiten und $50 \mathrm{~nm}$ hohen Ti-Droplets durch die Deposition von $\mathrm{ZrO}_{2}-$ Schichten mit jeweils ca. $115 \mathrm{~nm}$ Schichtdicke erkennen. Um einen momentanen Status des Glättungsverhaltens zu erlangen, wurde die Detailaufnahme aus Abbildung 5.12 in ein Grauwertbild umgewandelt und jede Ti-Schicht, welche hier als Marker für die Glättung fungiert, per Gauß-Fit angepasst und die jeweilige Amplitude ausgelesen (vgl. Abb. 5.13). 

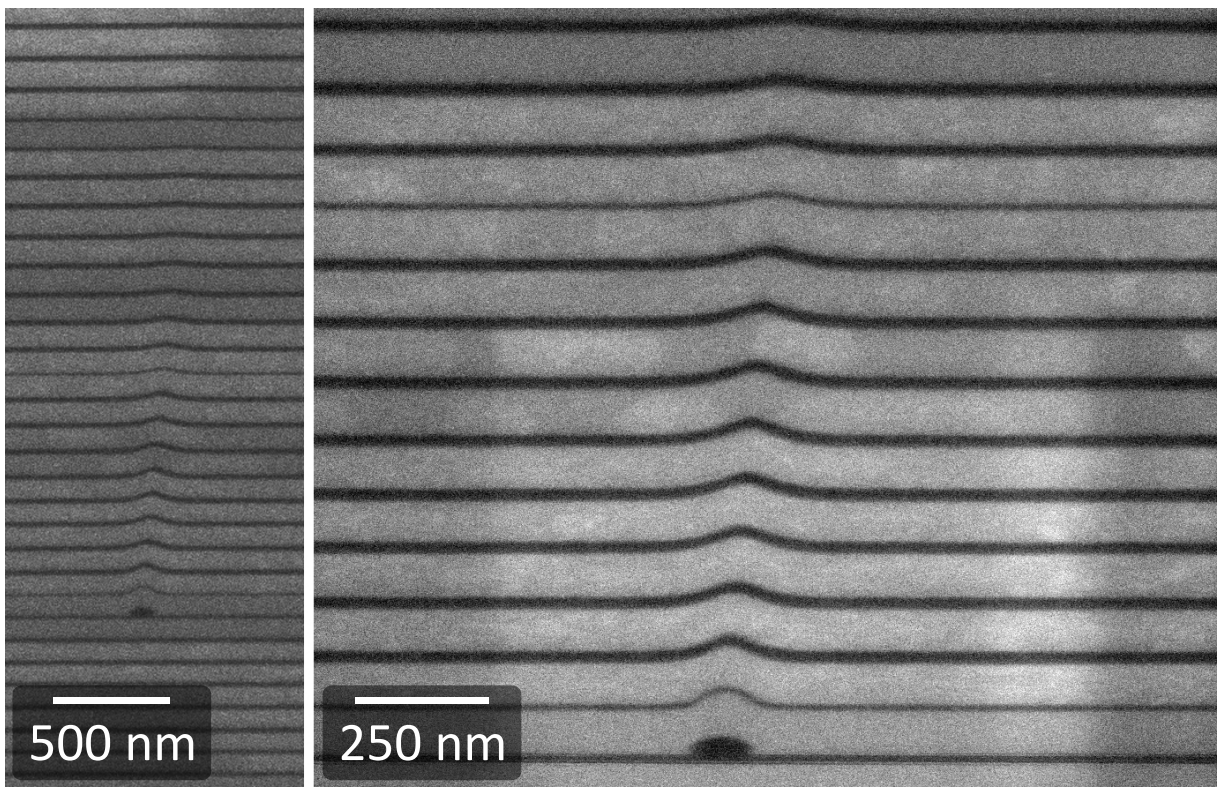

Abbildung 5.12: REM-Übersicht des Glättungsverhaltens von $\mathrm{ZrO}_{2}$ während einer $\mathrm{Ti} / \mathrm{ZrO}_{2}-$ Multilagendeposition. Die Störung der Schichtstruktur durch ein $100 \times 50 \mathrm{~nm}^{2}(\mathrm{~B} \times \mathrm{H})$ großes TiDroplet wird durch $\mathrm{ZrO}_{2}$ vollständig ausgeglättet [171]. (Die Aufnahme wurde freundlicherweise von Volker Radisch angefertigt.)

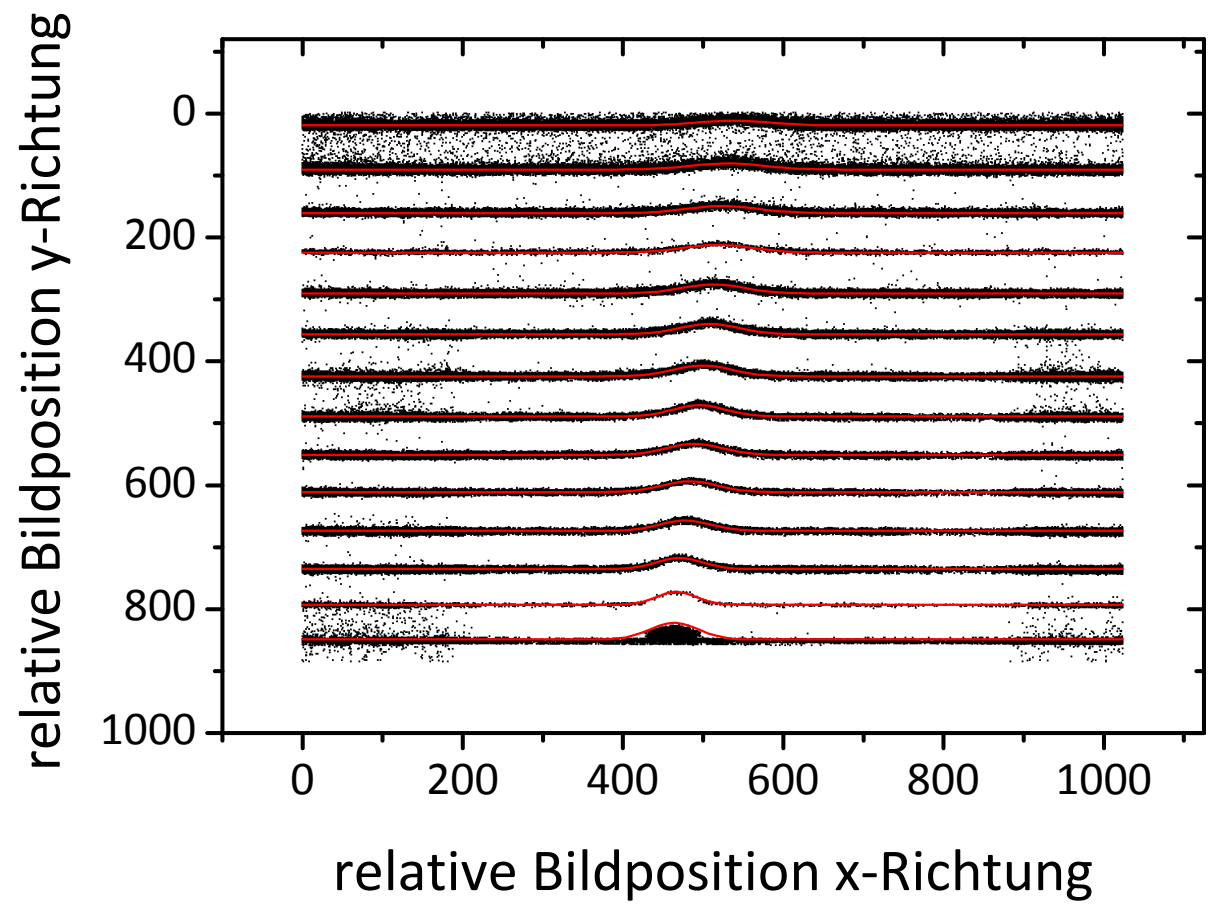

Abbildung 5.13: Untersuchung der Glättung eines Ti-Droplets in Abhängigkeit der $\mathrm{ZrO}_{2}-$ Schichtdicke. Dabei wurden die Amplituden der Ti-Schichten (schwarz) mittels Gauß-Fits, die Höhe des eigentlichen Droplets hingegen manuell ermittelt. 
Die Ausglättung des Droplets in Abhängigkeit der $\mathrm{ZrO}_{2}-$ Schichtdicke ist schließlich in Abbildung 5.14 dargestellt. Auch hier deutet sich ein zweistufiger Glättungsprozess an, bei dem vermutlich die Ausglättung hoher Frequenzen zu einer anfänglich starken Abnahme der Amplitude führt bis nur noch eine Hauptfrequenz übrig bleibt. Diese nimmt schließlich mit steigender $\mathrm{ZrO}_{2}$-Schichtdicke linear ab, bis die Störung der Schichtstruktur vollständig aufgehoben ist. Dazu ist wiederum eine sehr große Schichtdicke (ca. 2,2 $\mu \mathrm{m}$ ) vonnöten, um den langwelligen Anteil der Rauigkeiten auszuglätten [171].

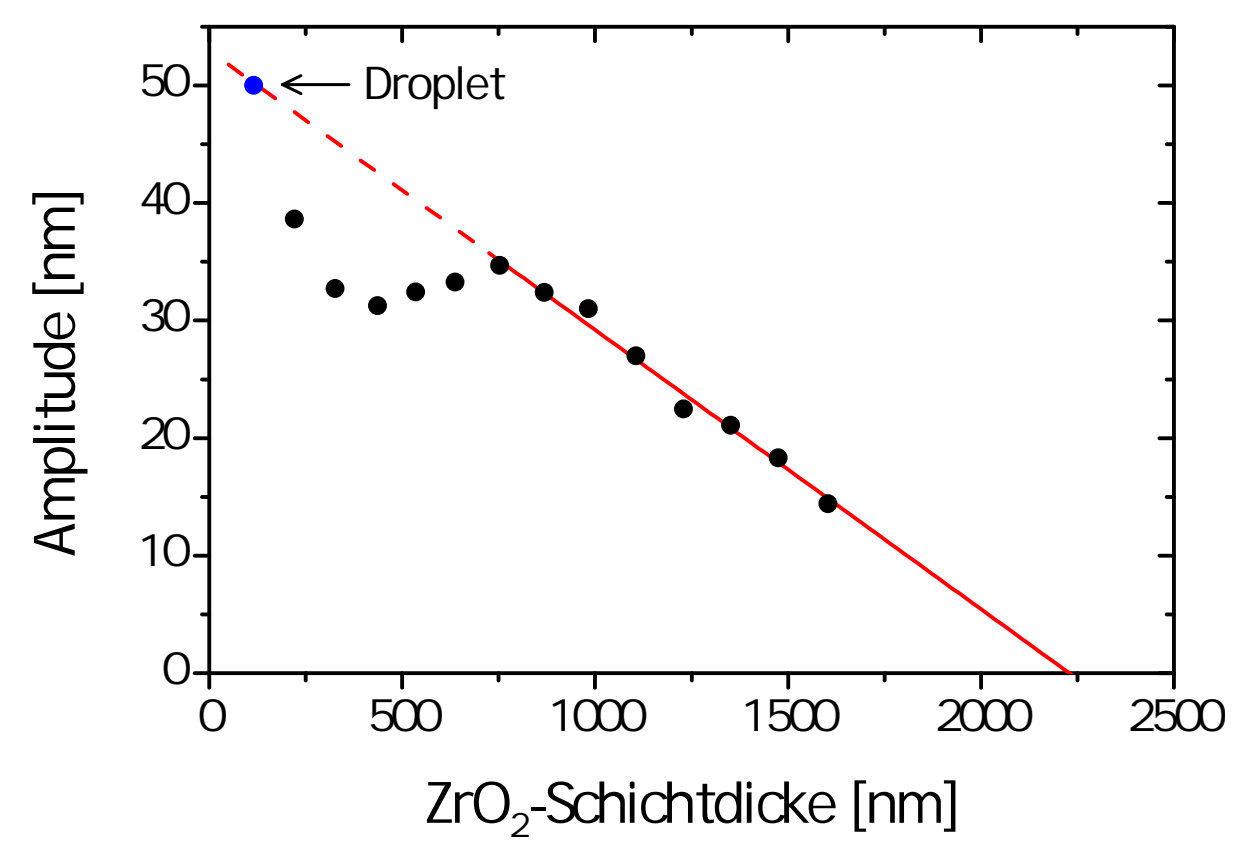

Abbildung 5.14: Auswertung des Glättungsverhalten von $\mathrm{ZrO}_{2}$ am Beispiel eines Ti-Droplets. Mit zunehmender $\mathrm{ZrO}_{2}$-Schichtdicke werden zuerst kleine Strukturgrößen ausgeglättet, bevor die Hauptfrequenz linear abnimmt. Für eine komplette Ausglättung ist die Deposition von mehr als $2 \mu \mathrm{m} \mathrm{ZrO}_{2}$ nötig.

Im Folgenden sollen Wachstumseigenschaften von $\mathrm{Ti}$ und $\mathrm{ZrO}_{2}$ anhand von TEMAufnahmen analysiert werden. In Abbildung 5.15 ist dazu eine Übersicht eines periodischen $\mathrm{Ti} / \mathrm{ZrO}_{2}-$ Schichtsystems gezeigt, bei dem sich die unterschiedliche Struktur der Einzelkomponenten erkennen lässt. Im Fall von Ti wurde bereits in Abbildung 5.7 dessen kristallines Wachstum bestätigt, was sich auch in dieser TEM-Aufnahme zeigt. Weiterhin ist hier zu sehen, dass die einzelnen Körner in Säulen aufwachsen, die bei den hier deponierten $50 \mathrm{~nm}$ dicken Ti-Schichten durchgängig von einer zur nächsten Grenzfläche reichen. Bei $\mathrm{ZrO}_{2}$ lässt sich hingegen keine Kristallstruktur erkennen, die Schichten sind komplett amorph gewachsen, wie es sich auch in der Literatur bei laserdeponiertem $\mathrm{ZrO}_{2}$ bei Raumtemperatur zeigt [172, 173]. Zudem 
deutet sich bei dem Wachstum der Multischichten keine Entstehung einer kumulativen Rauigkeit an, was neben der materialbedingten Glättung des $\mathrm{ZrO}_{2}$ außerdem durch die Deposition hochenergetischer Targetpartikel während der gepulsten Laserdeposition begründet [132] und für die Verwendung der Multischicht als MLL von besonderer Wichtigkeit ist.

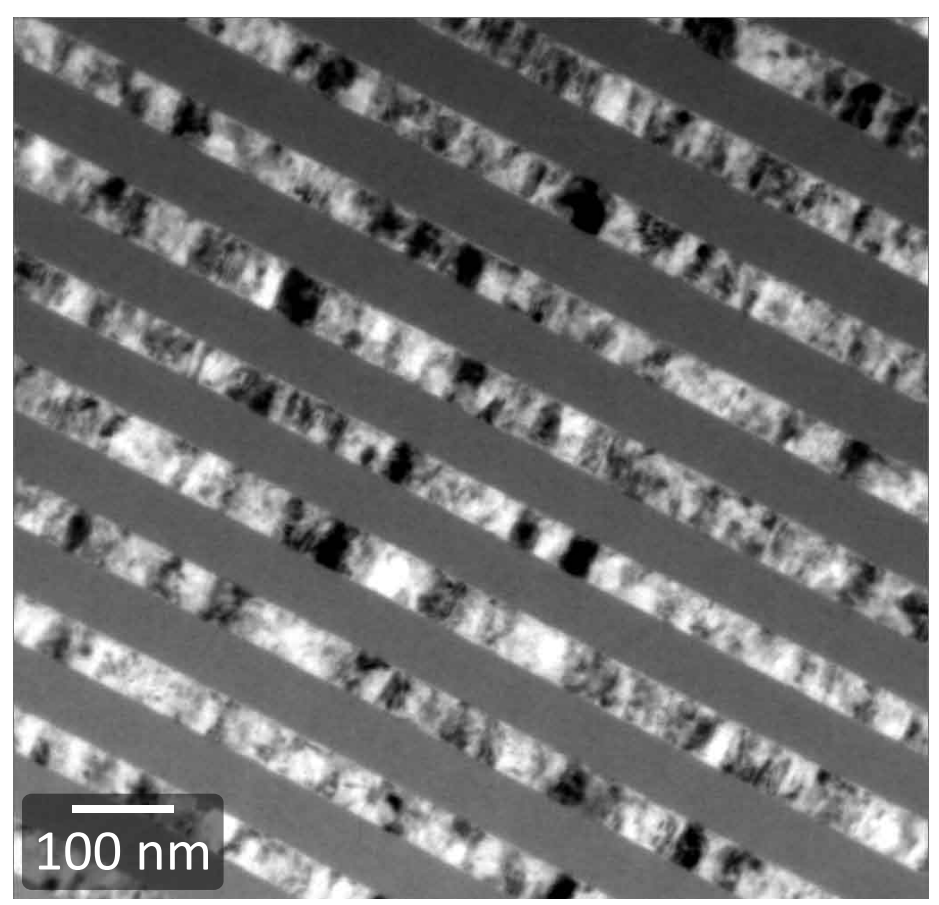

Abbildung 5.15: TEM-Übersichtsaufnahme eines periodischen $\mathrm{Ti} / \mathrm{ZrO}_{2}-$ Multischichtpakets mit ca. $50 \mathrm{~nm}$ Einzelschichtdicke. Während $\mathrm{ZrO}_{2}$ komplett amorph aufwächst, zeigt sich beim Wachstum des Ti eine säulenartige Kristallstruktur. (Die Aufnahme wurde freundlicherweise von Inga Knorr angefertigt.)

In Abbildung 5.16 sind zwei Grenzflächen des $\mathrm{Ti} / \mathrm{ZrO}_{2}-$ Schichtsystems im Detail dargestellt. Der Übergang von amorphem $\mathrm{ZrO}_{2}$ (links unten) zu kristallinem $\mathrm{Ti}$ (Mitte) bildet eine sehr scharfe Grenzfläche aus, welche zudem sehr glatt ist. Obwohl die einzelnen Körner des Ti verschiedene Orientierungen zeigen, scheint die zweite Grenzfläche von Ti zu $\mathrm{ZrO}_{2}$ ebenfalls sehr feinstrukturiert zu sein. Dabei sind die TiKristallite parallel zur Richtung des Schichtwachstums (von links unten nach rechts oben) ausgerichtet.

Bei der Betrachtung einer aperiodischen $\mathrm{Ti} / \mathrm{ZrO}_{2}-\mathrm{Multischicht} \mathrm{(s.} \mathrm{Abb.} \mathrm{5.17)} \mathrm{lässt}$ sich mit kleiner werdender Schichtdicke ein struktureller Wechsel von kristallinem Ti zur amorphen oder nanokristallinen Form erkennen. Dieser Übergang findet bei einer kritischen Schichtdicke kleiner als $10 \mathrm{~nm}$ statt und lässt sich im Detail anhand der 


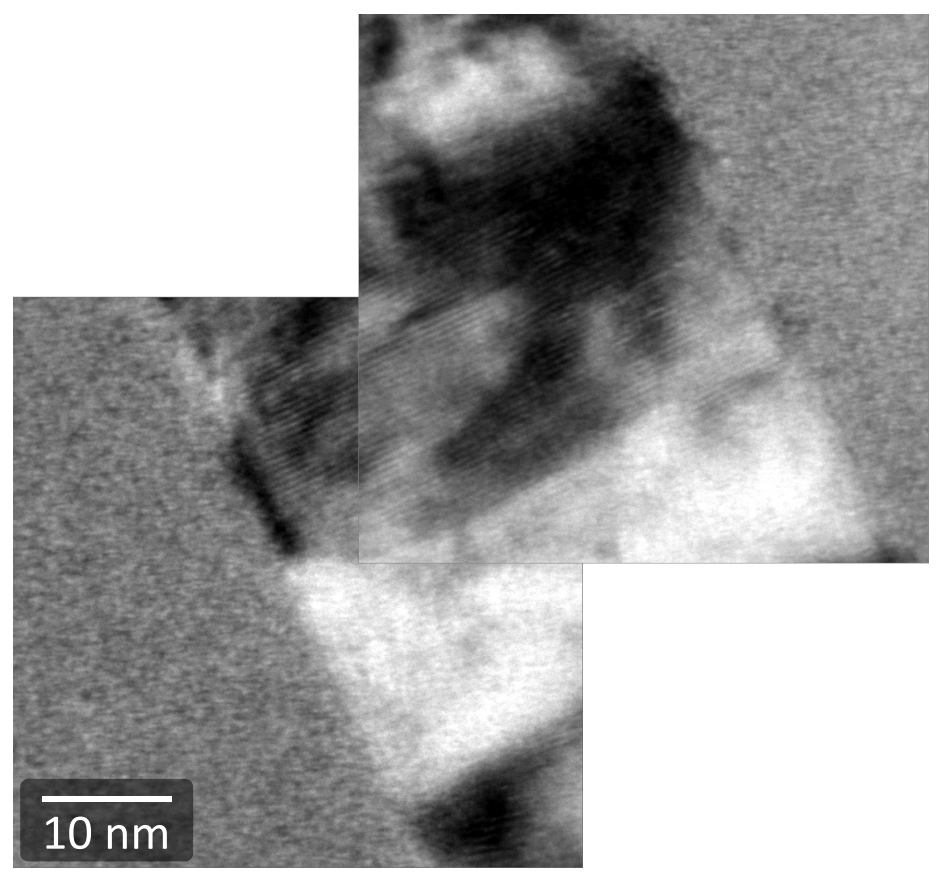

Abbildung 5.16: Detail-Aufnahme einer Ti/ZrO ${ }_{2}$-Grenzfläche im TEM. Der Übergang vom amorphen $\mathrm{ZrO}_{2}$ zum kristallinen $\mathrm{Ti}$ und umgekehrt vollzieht sich sehr scharf mit geringer Grenzflächenrauigkeit. Die Ti-Körner nehmen in der Höhe die komplette Schicht ein und sind senkrecht zu den Grenzflächen orientiert. Das Schichtwachstum erfolgte von links unten nach rechts oben. (Die Einzelaufnahmen wurden freundlicherweise von Inga Knorr angefertigt.)

vergrößerten TEM-Aufnahmen in Abbildung 5.18 verfolgen. Im linken Teil sind ca. $12 \mathrm{~nm}$ dicke Ti-Schichten gezeigt, deren einzelne Kristallite eine Abweichung vom Säulenwachstum zeigen und zum größten Teil nicht mehr senkrecht zur Grenzfläche ausgerichtet sind. Dadurch zeichnet sich ein leichter Anstieg der Grenzflächenrauigkeit ab. Im rechten TEM-Bild aus Abbildung 5.18 zeigen die ungefähr $9 \mathrm{~nm}$ dicken TiSchichten nur noch vereinzelte, fast runde Körner auf, die in einer amorphen oder nanokristallinen Matrix eingebettet sind. Die Abgrenzung der Einzelschichten bleibt dabei erhalten, sodass die Schichtstruktur auch bei geringeren Schichtdicken intakt bleibt. 


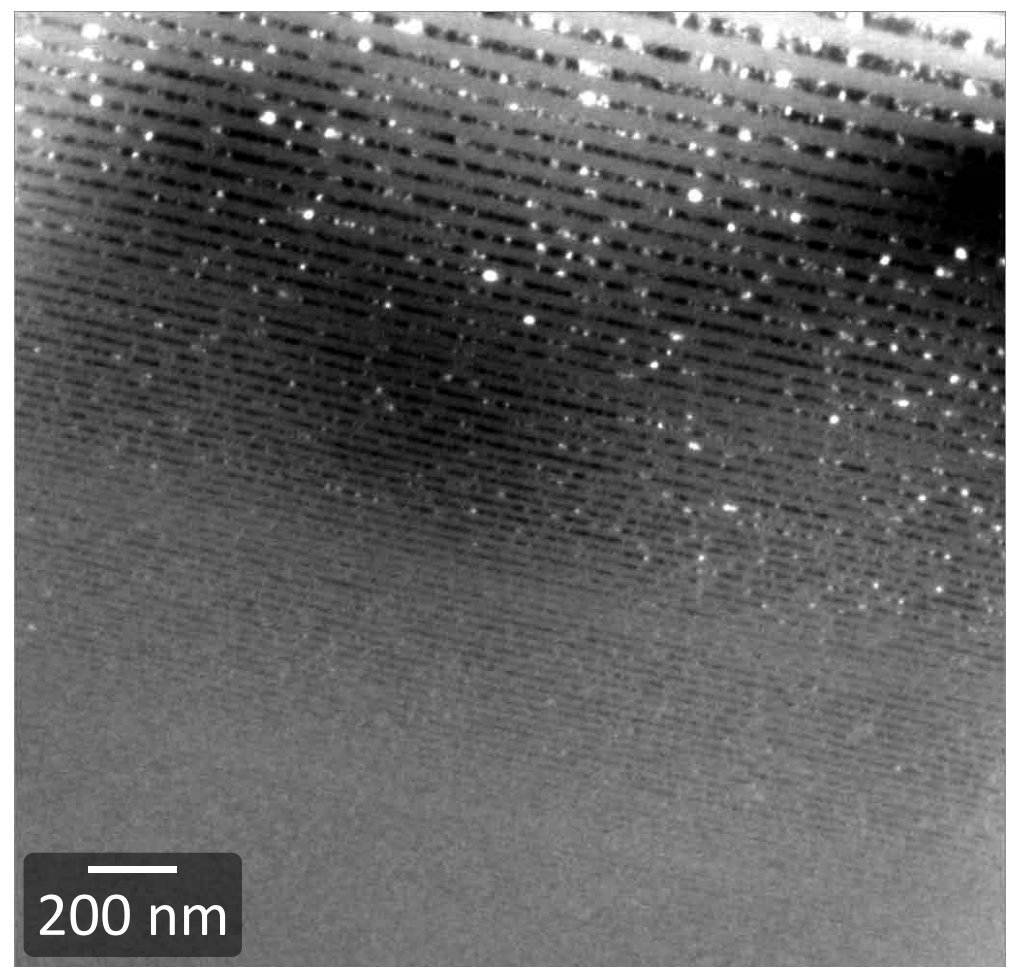

Abbildung 5.17: TEM-Untersuchungen an einer aperiodischen $\mathrm{Ti} / \mathrm{ZrO}_{2}-$ Multischicht. Während $\mathrm{ZrO}_{2}$ über den gesamten Schichtdickenbereich amorph aufwächst, zeigt das kristalline Ti unterhalb einer Dicke von $10 \mathrm{~nm}$ einen Übergang zu nanokristallinem oder ebenfalls amorphem Wachstum. (Die Aufnahme wurde freundlicherweise von Inga Knorr angefertigt.)
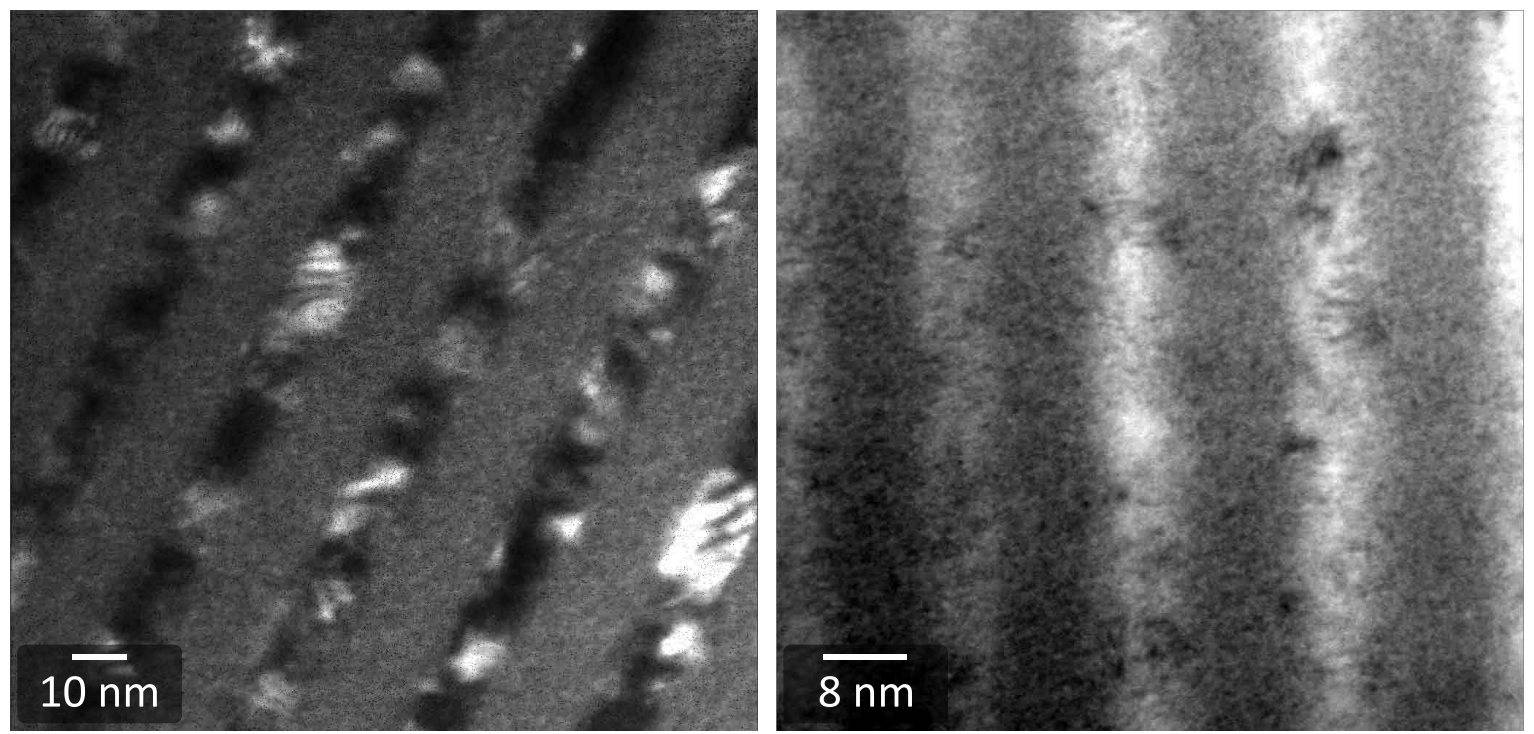

Abbildung 5.18: Detailaufnahmen von Ti-Schichten während des Wechsels von kristallinem zu amorphem oder nanokristallinem Wachstum. Dabei nimmt die Anzahl der Körner unterhalb einer Schichtdicke von $10 \mathrm{~nm}$ bis zur vollständigen Amorphisierung deutlich ab. (Die Aufnahmen wurden freundlicherweise von Inga Knorr angefertigt.) 


\subsubsection{MLL-Herstellung mittels PLD und FIB}

Anhand der vorangegangenen Untersuchungen zur Laserablation und -deposition von Ti und $\mathrm{ZrO}_{2}$ konnte die im Rahmen dieser Arbeit erste MLL-Teststruktur hergestellt werden. Zur experimentellen Vorbereitung wurden unmittelbar vor der Multischichtherstellung die Depositionsraten der Komponenten bestimmt, indem Einzelschichten hergestellt und deren Schichtdicken anhand von Profilometrie-Messungen ermittelt wurden. Bei einem Target-Substrat-Abstand von $42 \mathrm{~mm}$ wurden dazu 5000 Pulse $\mathrm{ZrO}_{2}$ und 15000 Pulse Ti auf Si(111)-Substrate abgegeben. Die gemessenen Depositionsraten beliefen sich bei $\mathrm{ZrO}_{2}$ auf $0,042 \mathrm{~nm} / \mathrm{Puls}$ und bei $\mathrm{Ti}$ auf $0,0043 \mathrm{~nm} / \mathrm{Puls}$, wonach die benötigten Pulszahlen für die MLL-Schichtdicken nach Tabelle 5.1 berechnet wurden. Mit diesen Depositionsraten wurden die Multischichten direkt im Anschluss auf ein Si(111)-Substrat deponiert.

Nach der Schichtherstellung wurde die Multischicht auf dem Probentisch im FIB platziert, um eine MLL nach dem im Folgenden beschriebenen Ablauf herzustellen. Sämtliche Prozessschritte wurden dabei freundlicherweise mit hohem Engagement von Volker Radisch am Institut für Materialphysik unterstützt und sind größtenteils in [174] veröffentlicht. Sofern nicht näher gekennzeichnet sind die folgenden Aufnahmen mittels des im FIB integrierten REM angefertigt worden. Ansonsten wurden Ionenstrahlen zur Abbildung benutzt. Für die geplante MLL mussten zwei Lamellen aus dem Substrat herausgeschnitten werden, die nach dem Herauslösen ungefähre Abmaße von $15 \times 1 \mu \mathrm{m}^{2}(\mathrm{~L} \times \mathrm{B})$ besitzen sollten. Im ersten Schritt wurde dazu die ausgewählte Substratposition mit einer Pt-Schutzschicht versehen, um die eigentliche Schicht bei späteren Schneidvorgängen durch den $\mathrm{Ga}^{+}$-Ionenstrahl nicht zu beschädigen oder mit Ga zu kontaminieren. Da die beiden Lamellen nebeneinander in einer Reihe präpariert werden sollten, wurde die Fläche der Pt-Deposition auf $40 \times 1,5 \mu \mathrm{m}^{2}$ festgesetzt (s. Fläche in Bildmitte aus Abb. 5.19 links). Mittels einer Gasinjektionsnadel wurde eine $\left(\mathrm{CH}_{3}\right)_{3} \mathrm{Pt}\left(\mathrm{C}_{\mathrm{p}} \mathrm{CH}_{3}\right)$-Verbindung in die Vakuumkammer eingelassen, welche nur dort als Pt-Schicht abgeschieden wird, wo der Elektronen- oder Ionenstrahl über eine definierte Substratfläche gerastert wird und eine chemische Reaktion des Gases hervorruft. Für die MLL-Präparation wurde mit dem Elektronenstrahl zunächst eine ca. $500 \mathrm{~nm}$ dicke Pt-Schicht mit einem Blendenstrom von 1,6 nA deponiert. Danach folgte eine Ionenstrahldeposition von Pt bis zu einer Gesamtschichtdicke von 5,1 $\mathrm{m}$ (entspricht etwas weniger als die Hälfte des geplanten beam stops), welche eine schnellere Wachstumsrate erlaubt, die unbehandelte Probenoberfläche aber beschädigt hätte. Dabei wurde als Faustregel ein Blendenstrom von $5-6 \mathrm{pA} / \mu \mathrm{m}^{2}$ angenommen. 

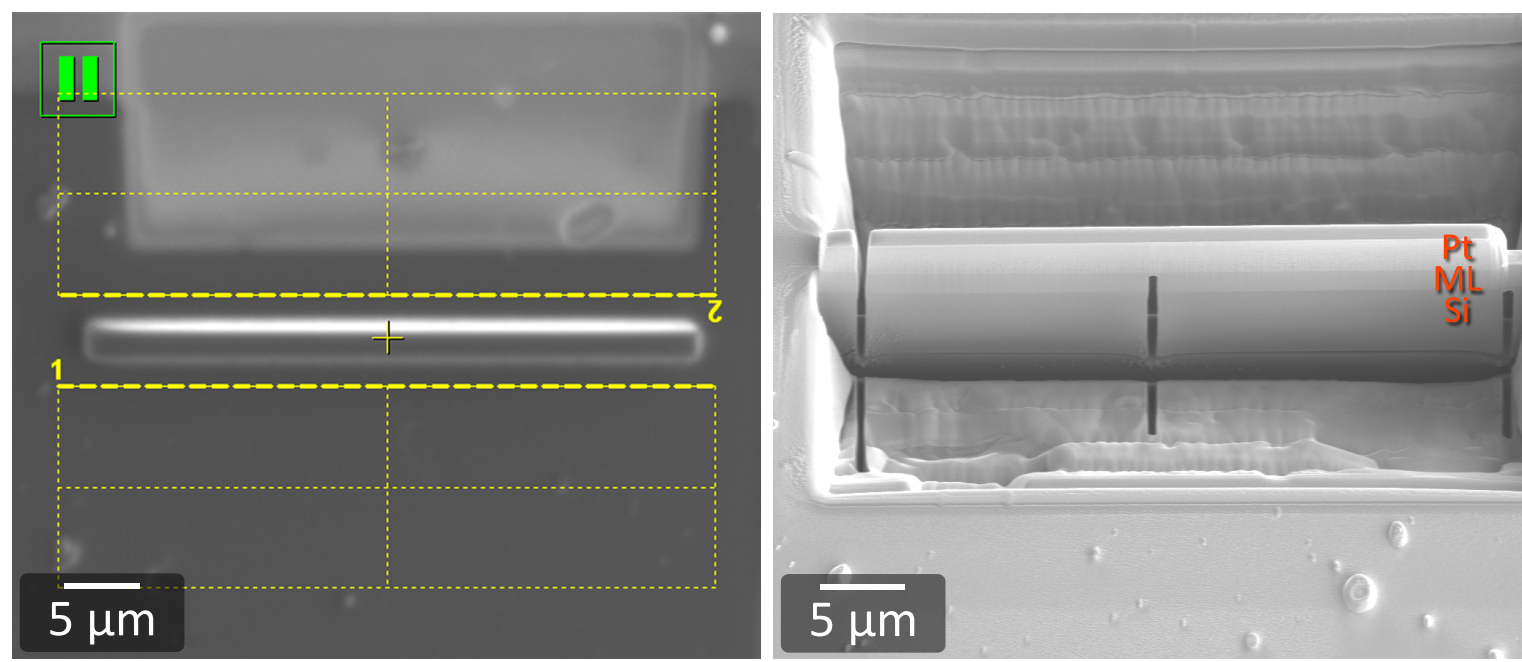

Abbildung 5.19: Erste Fertigungsschritte einer MLL mittels FIB. Nachdem eine $5,1 \mu \mathrm{m}$ dicke PtSchutzschicht elektronen- und ionenstrahldeponiert wurde (links, Ionenbild), wurden die Multilagen (ML) durch Materialabtrag ober- und unterhalb der Lamelle mit einem fokussierten $\mathrm{Ga}^{+}-$ Ionenstrahl freigelegt (rechts, Elektronenbild).

Im nächsten Schritt musste der Querschnitt des Schichtpakets freigelegt werden, indem ober- und unterhalb der Lamelle mit Hilfe des $\mathrm{Ga}^{+}-$Ionenstrahls Schichtmaterial bis zum Substrat hin abgetragen wurde. Dies erfolgte durch sog. „regular cross sections" (gelbe Pattern in Abb. 5.19 links), bei denen der Ionenstrahl senkrecht zur Substratoberfläche ein treppenstufenförmiges Profil mit dem tiefsten Punkt nahe der Lamelle schneidet. Für eine ausreichende Stabilität der späteren MLL wurde, wie in Abbildung 5.19 rechts zu sehen, tief in das Substrat hineingeschnitten, um dieses als unteres Ende der Linse zu benutzen. Da die Breite der rechteckigen Pattern der doppelten Tiefe der Multischicht entsprechen muss, um die später benötigten Schnitte vor dem Herausheben der Lamellen tätigen zu können, wurde zunächst ein relativ hoher Strahlstrom von $20 \mathrm{nA}$ gewählt. Nahe der Lamelle erfährt der Ionenstrahl in tieferen Regionen normalerweise eine Ablenkung zu seiner geraden Flugbahn, sodass die Breite bzw. Dicke der Lamelle nach unten hin trapezförmig zunimmt [175]. Dies kann durch eine sog. „regular cross section“ behoben werden, indem der Probentisch um $1,2^{\circ}$ relativ zum Ionenstrahl verkippt und die Lamelle so auf eine Dicke von $1 \mu \mathrm{m}$ nachgeschnitten wird. Die definierte Pattern-Fläche wird dabei linienförmig vom Ionenstrahl abgerastert, sodass der letzte Linienscan direkt an der Querschnittsfläche der Lamelle erfolgt. Nur so lässt sich eine Redeposition von abgetragenem Material auf die Lamelle vermeiden. Unter einem Offset-Winkel von $45^{\circ}$ zur $\mathrm{Ga}^{+}-$Kanone wurden im Anschluss mit einer $1 \mathrm{nA}$ Ionenstrahlblende drei zur Schichtstruktur senkrechte 
und ein waagrechter Schnitt im Substrat getätigt (sog. „Doppel-U-cut“), um die zwei Lamellen voneinander zu separieren und auf das Herauslösen vorzubereiten. Wie in Abbildung 5.19 rechts gezeigt, sind die Querschnittslamellen zu diesem Zeitpunkt lediglich am äußeren rechten Rand mit dem Substrat verbunden.

Nach einer Pt-Kontaktierung der ersten Lamelle an einer beweglichen OmniprobeSpitze (s. Abb. 5.20 links) konnte die Lamelle vom Substrat mit einem weiteren Schnitt abgetrennt werden. Die Lamelle wurde schließlich durch punktuelle Pt-Deposition an den Auflageflächen eines im FIB stehenden $\mathrm{Cu}-$ Halters fixiert und von der Omniprobe mit einem dünnen Schnitt abgelöst. Freistehend ist sie in Abbildung 5.20 rechts dargestellt.
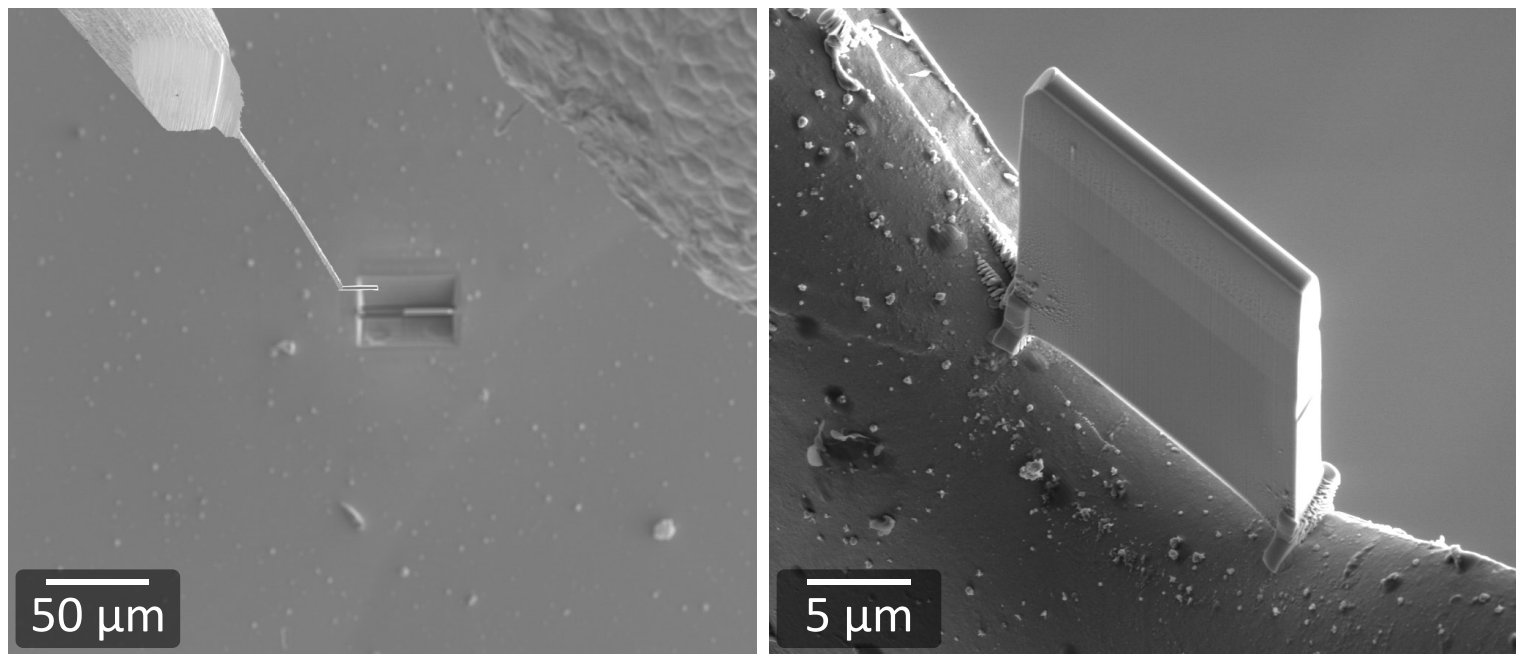

Abbildung 5.20: Ausheben der ersten Lamelle (links) und Transfer zu einem Cu-Halter (rechts). Mit dem $\mathrm{Ga}^{+}$-Ionenstrahl wurde dabei einerseits die Verbindung zum Probenhalter durch Pt-Deposition hergestellt und andererseits der Kontakt zur Omniprobe-Spitze durch einen fokussierten Schnitt getrennt.

Die zweite Teillamelle (s. Abb. 5.21 links) wurde im nächsten Schritt auf gleiche Weise mit der Omniprobe-Spitze kontaktiert und herausgehoben. Da der Mikromanipulator nicht rotiert werden kann, musste die Drehung der Lamelle um die notwendigen $180^{\circ}$ in mehreren Schritten vollzogen werden. Als erstes wurde die Lamelle an einen liegenden $\mathrm{Cu}$-Probenhalter befestigt, welcher nach Belüften der Präparationskammer um $90^{\circ}$ gedreht senkrecht auf dem Probentisch fixiert wurde. Die nun waagrecht liegende Lamelle (s. Abb. 5.21 rechts) wurde mittels der Omniprobe vom $\mathrm{Cu}$-Halter abgenommen und dieser nach einem weiteren Belüftungsschritt wieder liegend auf dem Probentisch angebracht. Im Anschluss wurde die liegende Lamelle von dem Mikromanipulator abgelöst und am Halter angeklebt, bevor die Vakuumkammer ein 

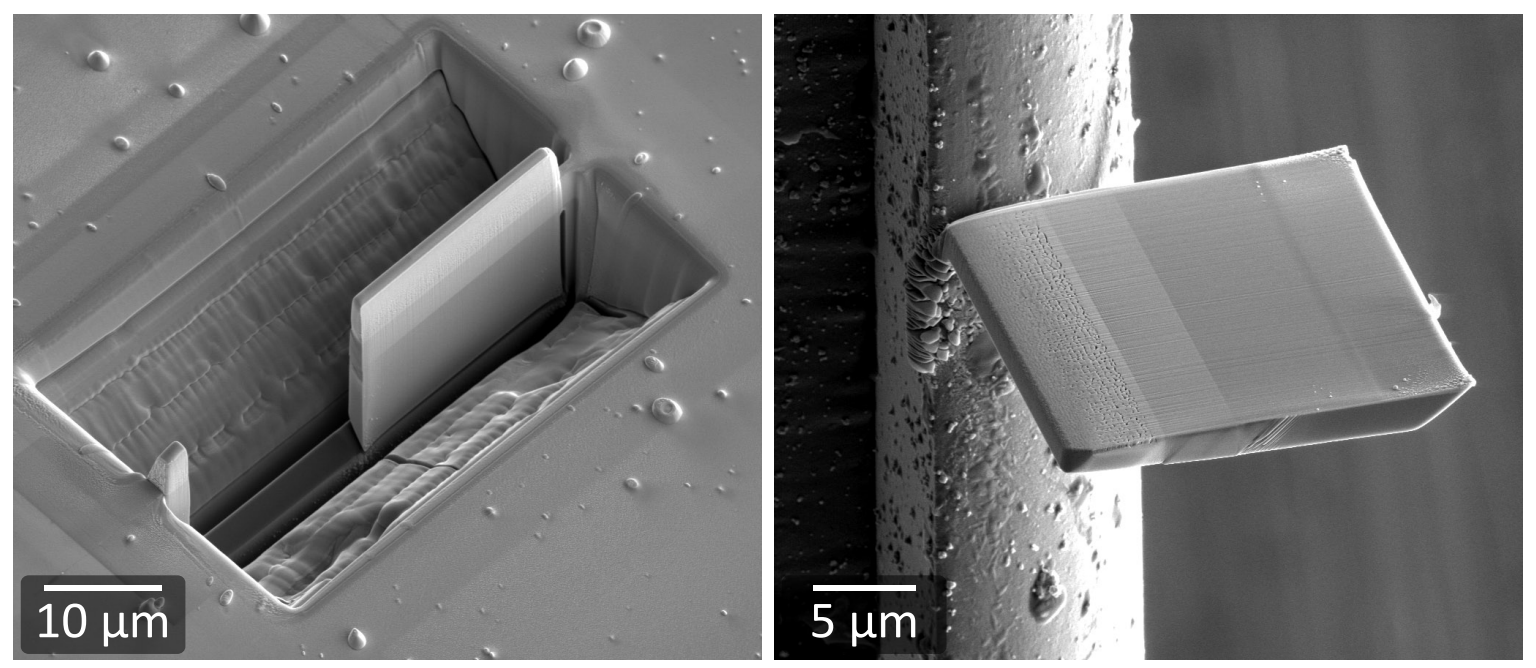

Abbildung 5.21: REM-Aufnahme der zweiten Lamelle vor dem Herauslösen aus dem Substrat (links) und nach der waagrechten Fixierung an einen $\mathrm{Cu}$-Halter (rechts). Insgesamt musste sie in mehreren Schritten um $180^{\circ}$ gedreht werden (s. Beschreibung im Text).

weiteres Mal belüftet werden musste. Durch erneute Drehung des $\mathrm{Cu}-\mathrm{Halters}$ um $90^{\circ}$ und Anbringen an die Omniprobe konnte eine Gesamtrotation der Lamelle um $180^{\circ}$ realisiert werden.

Als nächstes wurden die zwei Lamellen, eine am $\mathrm{Cu}$-Probenhalter und eine kopfstehend an der Omniprobe befestigt, vorsichtig aneinander angenähert und durch PtDeposition verschweißt. Abbildung 5.22 zeigt die zwei Lamellen, die sich bereits in einem geringen Abstand zueinander befinden. Bei dieser Justage muss sehr genau auf einen definierten Abstand (Größe des beam stops), Parallelität und Höhe (Versatz aus der Zeichenebene heraus) der Lamellen geachtet werden. Gerade der letztere Parameter ist im Elektronenbild nur grob anhand der Schärfentiefe einstellbar, sodass auch hier wieder synchron mit ionenerzeugten Bildaufnahmen gearbeitet werden musste.

Nach der Kontaktierung folgte das sukzessive Ausdünnen der noch $1 \mu \mathrm{m}$ dicken Doppel-Lamelle bis zu einer geplanten optischen Tiefe von $200 \mathrm{~nm}$. Die Ionenstrahlströme wurden dabei während regelmäßiger Prüfung der Tiefe mittels REM schrittweise von $1 \mathrm{nA}$ auf $0,1 \mathrm{nA}$ reduziert, bis im letzten Schritt die Seitenflächen der MLL mit einem minimalen Strahlstrom von $30 \mathrm{pA}$ glatt poliert wurden. Abbildung 5.23 zeigt schließlich die fertig ausgedünnte MLL in der Seitenansicht, bei der Substrat, Multischicht und Pt-beam stop klar voneinander unterscheidbar sind. An der zusammengefügten Position der beiden Teillinsen wurde zur zusätzlichen Stabilisierung nachträglich eine weitere dünne Pt-Schicht ionenstrahldeponiert. Die Ausmaße der auf diese Weise fabrizierten MLL betragen $15 \times 18 \times 0,2 \mu \mathrm{m}^{3}(\mathrm{~B} \times \mathrm{L} \times \mathrm{T})$. 


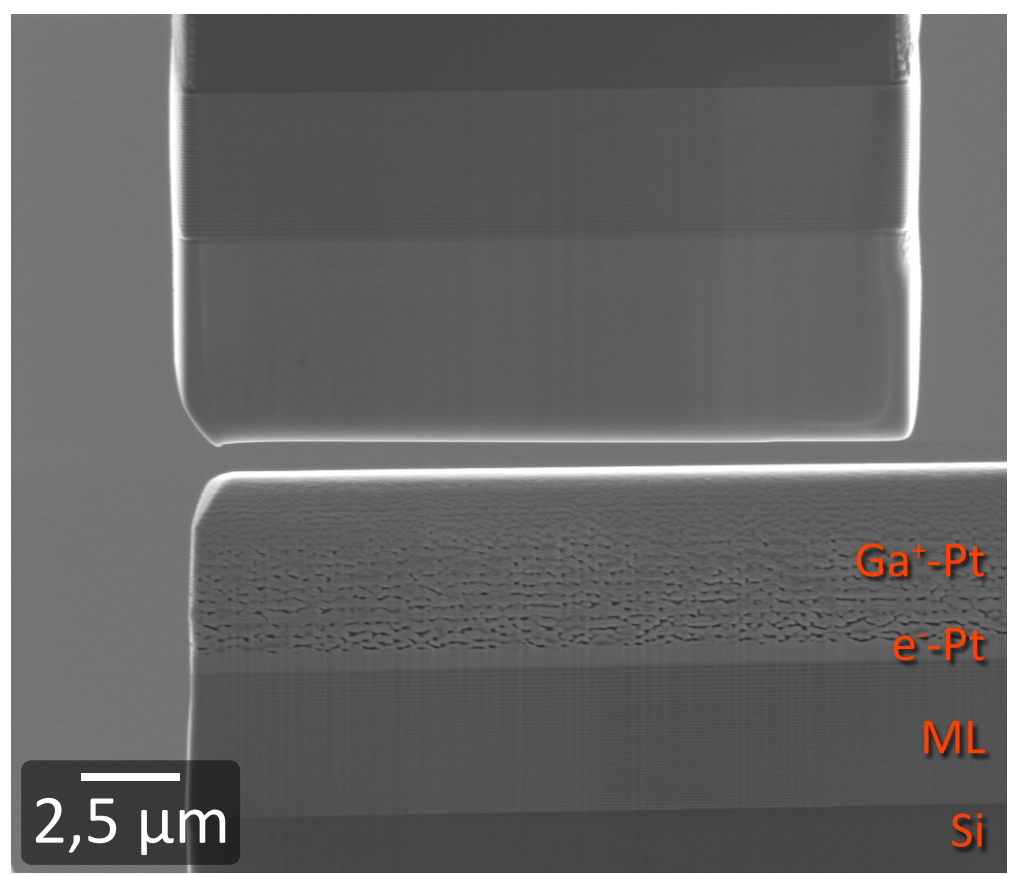

Abbildung 5.22: Behutsame Annäherung der zwei Teillinsen durch Verfahren des Probentisches und der Omniprobe. Besonders musste dabei auf eine parallele Ausrichtung der Schichtsysteme zueinander, den richtigen Abstand zwischen den beiden Lamellen (beam stop) und deren richtige Höhe (in die Zeichenebene hinein) geachtet werden. Letzteres ist ohne gleichzeitige Überwachung per Ionenbild kaum möglich.

Zum Abschluss wurde die MLL auf einem $\mathrm{Si}_{3} \mathrm{~N}_{4}$-Fenster fixiert, um zusätzlich einen äußeren beam stop zu erzeugen, welcher den experimentellen Aufbau am „,tabletop“-Röntgenmikroskop vereinfacht. Somit wird ein ausschließliches Beleuchten der MLL garantiert, ohne eine zusätzliche Lochblende in den Strahlengang integrieren zu müssen. Dazu wurde ein $\mathrm{Si}_{3} \mathrm{~N}_{4}$-Substrat mit einem ausgedünnten Bereich von $100 \mathrm{~nm}$ Dicke verwendet, welches für eine ausreichende Absorption der Röntgenstrahlung mit einer $530 \mathrm{~nm}$ dicken $\mathrm{Au}$-Schicht mittels Sputterdeposition versehen wurde. In den gedünnten Bereich wurde mittels FIB ein quadratisches Loch von $16 \times 16 \mu \mathrm{m}^{2}$ mit 20 nA Strahlstrom geschnitten, die MLL mittels der Omniprobe direkt über dem Loch eingefasst und durch Pt-Deposition fixiert. Um zu überprüfen, ob sich die Multischichten mittig über dem geschnittenen Loch befinden, wurde das $\mathrm{Si}_{3} \mathrm{~N}_{4}$-Substrat um $180^{\circ}$ gedreht und erneut im FIB betrachtet (s. Abb. 5.24). Die fertiggestellte MLL ist fest mit dem makroskopischen Substrat verbunden und für die geplanten Experimente zur Fokusbestimmung einfach zu handhaben. Die gesamte benötigte FIB-Zeit zur Herstellung der MLL belief sich hier auf ca. 8 Stunden. 


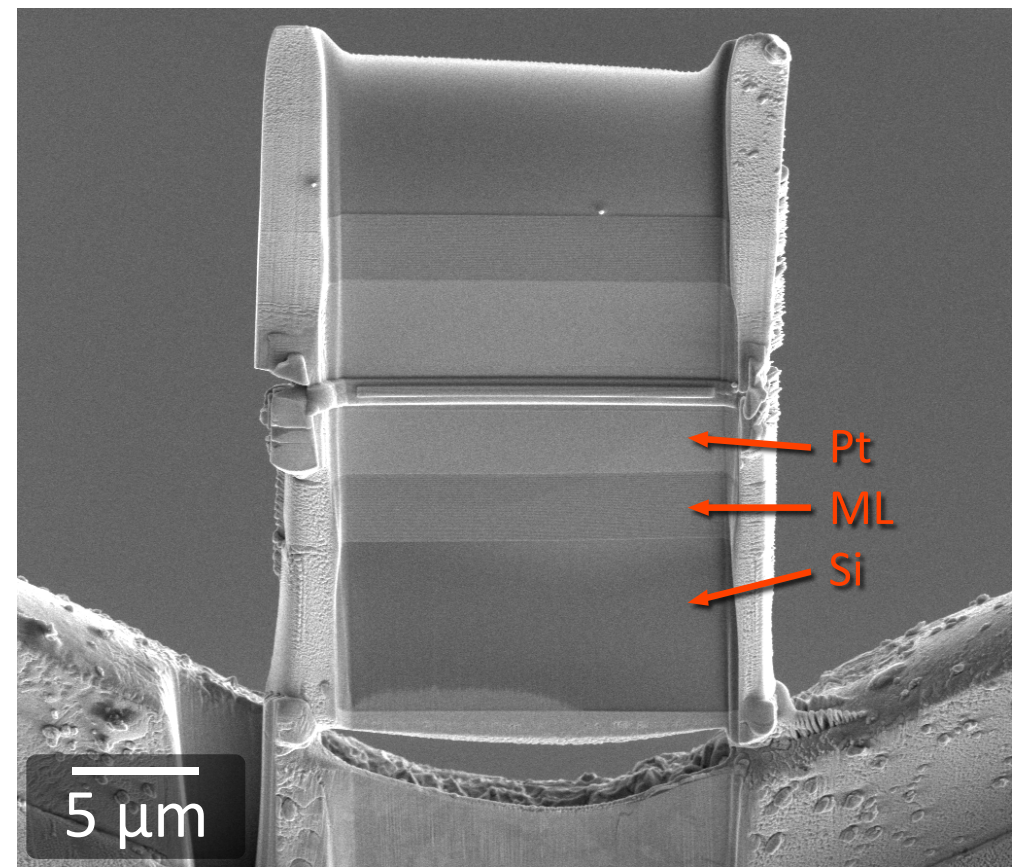

Abbildung 5.23: Seitenansicht der auf $200 \mathrm{~nm}$ optische Tiefe fertig ausgedünnten MLL im FIB. Die Verbindung der beiden Teillinsen in der Bildmitte wurde im Nachhinein durch weitere PtIonenstrahldeposition von beiden Seiten verstärkt.

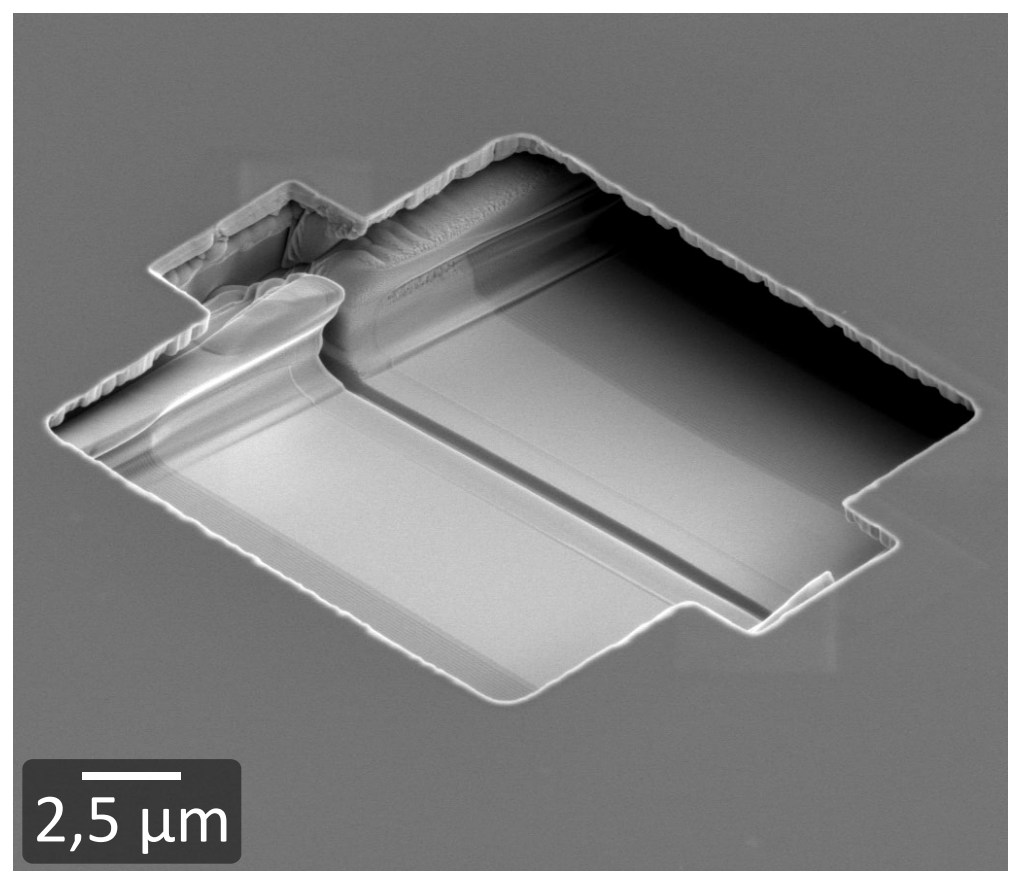

Abbildung 5.24: Einbettung der MLL auf ein zuvor geschnittenes Loch eines $\mathrm{Si}_{3} \mathrm{~N}_{4}$-Substrats zur Generierung eines äußeren beam stops. Die Linse ist durch Pt-Deposition fest mit dem Substrat verbunden und kann leicht im Strahlengang des „table-top“-Röntgenmikroskops justiert werden. 


\subsubsection{Strahlcharakteristik am „tabletop“-Mikroskop}

Zur Untersuchung der Fokussierungseigenschaften wurde die auf dem 1,5 × 1,5 $\mathrm{mm}^{2}$ großen $\mathrm{Si}_{3} \mathrm{~N}_{4}$-Trägersubstrat befindliche MLL in die in Kapitel 3.3 beschriebene Mikroskopie-Anlage im Laser-Laboratorium Göttingen e. V. eingebaut und von Michael Reese mittels Beleuchtung quasi-monochromatischer Röntgenstrahlung analysiert sowie deren optischen Eigenschaften mit zwei unterschiedlichen Experimenten (s. Kap. 5.1.1) vermessen [157,162]. Für die Untersuchung der Strahlcharakteristik im Fernfeld wurden bei verschiedenen Detektorpositionen im Abstand von 5-85 mm zur Linse Intensitäten der 0. und 1. Beugungsordnung aufgenommen, um Rückschlüsse auf die Strahlpropagation am Ort des Fokus zu ziehen. In Abbildung 5.25 ist dazu eine Auswahl an Beugungsbildern der transmittierten Röntgenstrahlung gezeigt, bei denen die zentrale 0. Beugungsordnung entlang der optischen Achse und die divergierenden 1. Beugungsordnungen erkennbar sind. Aus der Verbreiterung der 1. Ordnungen und deren jeweiliger Abstand zur optischen Achse lassen sich anhand des Beugungswinkels $\alpha$ und des Divergenzwinkels $\theta$ sowie der Kenntnis der Aperturgröße (aktiv beleuchtete MLL-Fläche) drei verschiedene Berechnungen für die Bestimmung der Fokuslänge $f$ durchführen. Durch die Anwendung der Strahlensätze ergibt sich mit dem Beugungswinkel und der Aperturgröße im ersten Auswertungsschritt eine Fokuslänge von $f=177 \mu \mathrm{m}$. Unter Berücksichtigung des Divergenzwinkels, der hier mit 20,4 mrad abgeschätzt wurde, berechnet sich die Fokuslänge ebenfalls anhand Überlegungen aus dem Strahlensatz zu $f=224 \mu \mathrm{m}$. Bei der dritten Methode wurde die Brennweite unter der Annahme einer Gaußschen Strahloptik mittels Einbeziehung der Rayleigh-Länge angegeben, welche den Abstand vom Fokus entlang der optischen Achse beschreibt, ab der sich die Fokusgröße verdoppelt hat. Anhand dieses Ansatzes unter Berücksichtigung der Verbreiterung des 1. Beugungsmaximums sowie des Divergenzwinkels lässt sich die Brennweite $\mathrm{zu} 220 \mu \mathrm{m}$ berechnen, welche in guter Übereinstimmung mit der eingangs erwähnten Design-Fokuslänge steht (vgl. Kap. 5.1.1). Eine Kalkulation der Beugungseffizienz der MLL ergab sich anhand des Vergleichs der Intensitäten der 0. und 1. Beugungsordnung zu ca. 7,5\%. Dies stellt einen recht hohen Wert verglichen mit der Effizienz einer theoretischen Absorptionszonenplatte von $10 \%$ dar.

Weiterhin lässt sich anhand der letzten Methode die Abmessung der Strahltaille am Ort des Fokus (Auflösung) unter der Annahme eines Gauß-Strahls zu $R=180 \mathrm{~nm}$ ermitteln. Die Abweichung von der berechneten Fokusgröße von $R=105 \mathrm{~nm}$ kann durch mechanische Instabilitäten des Messaufbaus bezüglich Vibrationen oder zeitliche Instabilitäten der Röntgenquelle bezüglich Ort und Intensität der Punktquelle hervor- 


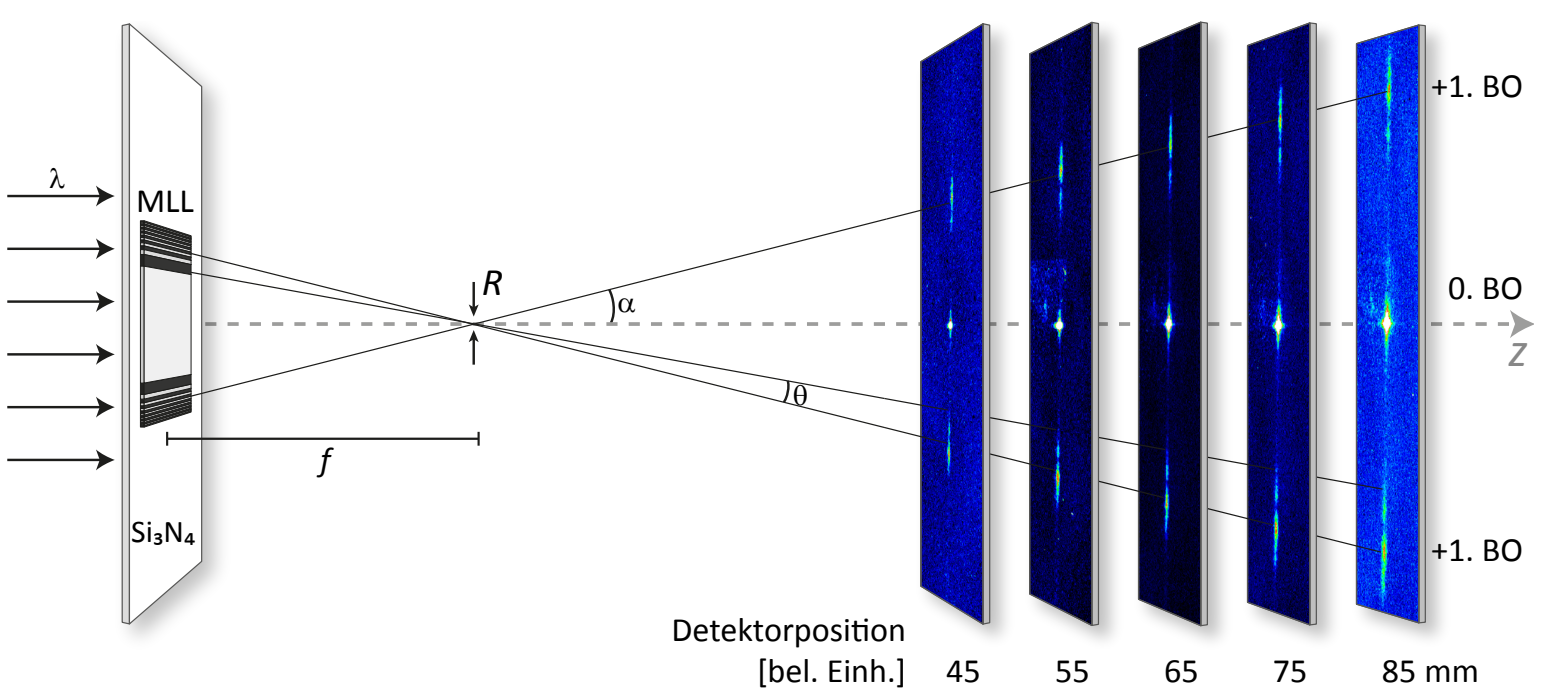

Abbildung 5.25: Fernfeld-Strahlpropagation der vermessenen MLL bei verschiedenen Detektorpositionen. Anhand der Verbreiterung der 1. Beugungsordnung sowie deren Abstände zur optischen Achse lassen sich Brennweiten von $170-224 \mu \mathrm{m}$ und eine Fokusgröße von $R=180 \mathrm{~nm}$ berechnen. Gerade bei großen Detektorabständen lässt eine Feinstrukturierung der 1. Beugungsordnung Messoder Herstellungsfehler erkennen. (Die Beugungsbilder wurden freundlicherweise von Michael Reese zur Verfügung gestellt und sind in $[157,176]$ veröffentlicht. Zur Übersichtlichkeit ist die MLL und deren Brennweite stark vergrößert dargestellt.)

gerufen werden. Ein wesentlicher Grund wird allerdings in einer herstellungsbedingten Abweichung der Einzelschichtdicken der MLL zum Zonenplattenbildungsgesetz gesehen. Durch eine abnehmende Depositionsrate während der Herstellung sind die dickeren, zuletzt deponierten Schichten etwas dünner als geplant. Dieser Eindruck ist bereits während der Fabrikation der MLL im FIB entstanden und deutet bei den hier durchgeführten Charakterisierungen auf eine starke Feinstruktur der 1. Beugungsordnung hin. Neben diesen Schichtdickenungenauigkeiten kann auch eine ungenaue Positionierung der beiden Einzellinsen zueinander als mögliche Fehlerquelle gesehen werden. In Abbildung 5.26, welche einen Linienscan der 1. Beugungsordnung zeigt, ist aus diesen Gründen anstatt eines definierten Gauß-Peaks die Überlagerung mehrerer nicht zu separierender Einzelfoki zu sehen, welche durch abweichende Zonenbreiten hervorgerufen wird. Um einen experimentellen Einfluss der Labor-Röntgenquelle auszuschließen, ist hier zusätzlich das Ergebnis einer Synchrotron-Messung der MLL gezeigt. Diese Untersuchungen mit weicher Röntgenstrahlung der Wellenlänge 2,88 nm wurden an der Synchrotronstrahlungsquelle BESSY II, Berlin von Dong-Du Mai (Courant-Forschungszentrum „Nano-Spectroscopy and X-Ray Imaging“, Universität Göttingen) und Thomas Gorniak sowie Tobias Senkbeil (AG Rosenhahn, Universität Heidelberg) durchgeführt. Der aus beiden Messungen erhaltene Nebenpeak, der nur 
auf einer Seite des Hauptmaximums sehr prägnant auftritt, ist ebenfalls bei den Untersuchungen von YAN et al. an einer nicht perfekten MLL für harte Röntgenstrahlung gesehen worden [95]. Experimentell und theoretisch ist von ihnen bestätigt worden, dass eine Abweichung der Schichtdicken vom Zonenplattenbildungsgesetz zu einer Verbreiterung des Fokus durch Interferenzerscheinungen auf einer Seite des Fokusprofils führt. Demnach tritt aufgrund destruktiver Interferenz eine unangemessene Variation der resultierenden Phase in Form von Nebenmaxima auf.

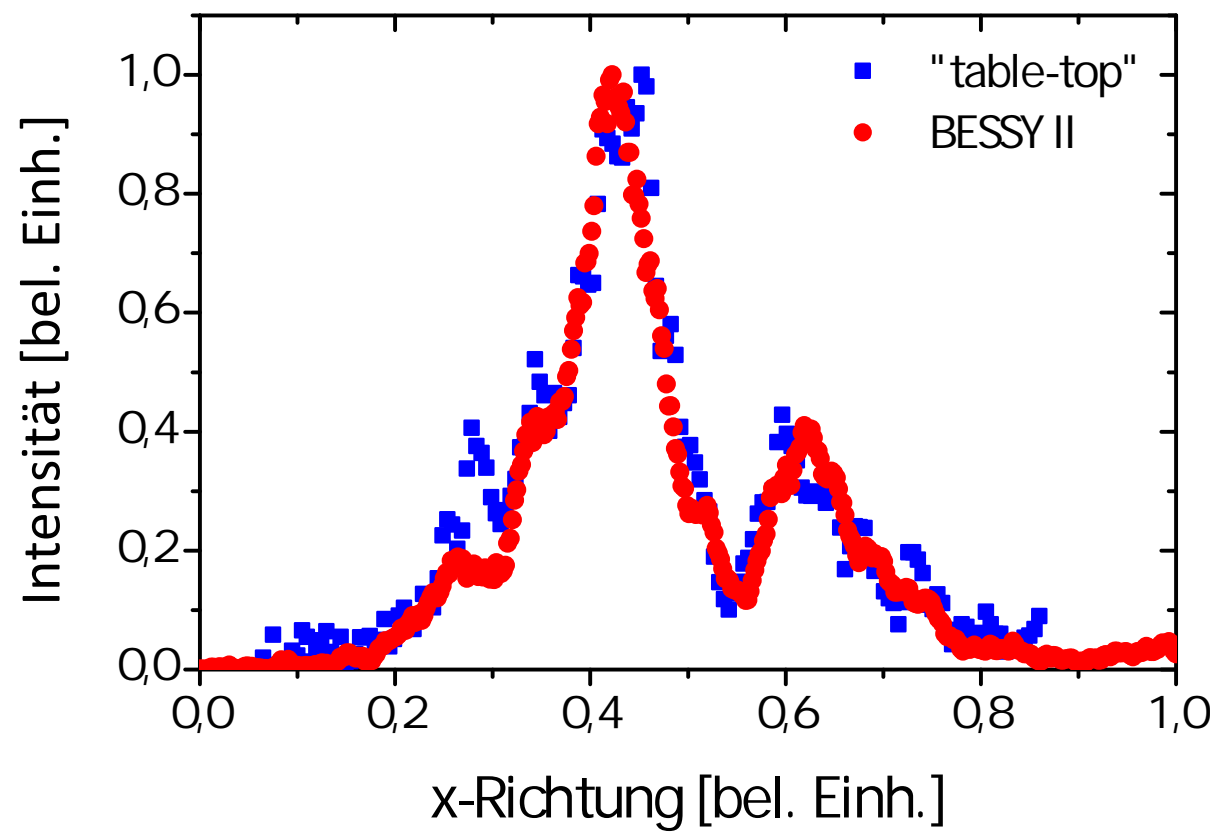

Abbildung 5.26: Linienprofil der 1. Beugungsordnung der MLL im Fernfeld, aufgenommen mit der „table-top“-Strahlungsquelle sowie an der Synchrotronstrahlungsquelle BESSY II. Die in beiden Messungen sichtbare Feinstrukturierung mit dem Auftreten eines Seitenmaximums (rechts) spricht für eine vom Zonenplattenbildungsgesetz abweichende Positionierung der MLL-Zonen. (Die Daten wurden freundlicherweise von Michael Reese und Dong-Du Mai zur Verfügung gestellt [162].)

Neben diesen Fernfeld-Untersuchungen wurde die Strahlpropagation durch die MLL in einem zweiten Experiment von Michael Reese mittels „knife-edge“-Messungen unmittelbar in der Nähe des Fokus analysiert. Diese sog. Schneidkanten-Abrasterung beruht auf einer lokalen sukzessiven Intensitätsabschattung einer Beugungsordnung, indem eine dünne Kante in verschiedenen Abständen relativ zur Fokusposition auf der optischen Achse jeweils senkrecht durch das Strahlprofil gefahren wird. Im Experiment wurde dazu ein FeC-Draht mit einem Durchmesser von $10 \mu \mathrm{m}$ verwendet, der über piezo-gesteuerte Linearmotoren mit einer Schrittweite von $50 \mathrm{~nm}$ verfahren werden kann. Somit lassen sich die Größen der Strahltaillen rund um den Fokus durch integrale Intensitätsabfälle erfassen. Zur genauen Ausrichtung der Drahtachse parallel zum 
Linienfokus der MLL wurde die schwach durch den gedünnten Bereich des $\mathrm{Si}_{3} \mathrm{~N}_{4}-$ Substrats hindurchtretende Röntgenstrahlung benutzt. Bereits im FIB wurde auf eine parallele Positionierung der MLL relativ zu den 1,5 mm langen Kanten des gedünnten Bereichs geachtet, sodass diese zur späteren Feinjustage im Fokusexperiment verwendet wurden. Aufgrund der im vorigen Abschnitt gewonnenen Erkenntnisse bezüglich einer möglichen inkorrekten Anordnung der beiden Teillinsen im FIB wurde für dieses Experiment nur eine der beiden Teillinsen berücksichtigt.

Beispielhaft ist in Abbildung 5.27 der Intensitätsverlauf jener „knife-edge“-Messung in Abhängigkeit der Kantenposition senkrecht zur optischen Achse gezeigt, die entlang der optischen Achse der Fokusposition am nächsten ist. Von links nach rechts betrachtet wurde die Schneidkante immer weiter in den gebeugten Strahl 1. Ordnung gefahren, sodass die Intensität schließlich bis zum Hintergrundrauschen unterdrückt werden konnte. Zur Auswertung ist eine numerische Ableitung des Intensitätsverlaufs gebräuchlich, aus welcher sich die Fokusgröße erschließt, wenn die Strahlungsintensität in Propagationsrichtung auf $1 / \mathrm{e}^{2}$ abfällt [177]. Da es bei der hier vorliegenden Feinstruktur der 1. Beugungsordnung der Fall sein könnte, dass dieser 1/e $\mathrm{e}^{2}$-Abfall mehrmals durchlaufen wird, bietet sich die rauscharme Methode der Bildmomente an, durch welche generell die Fokussierbarkeit von Lasern beschrieben wird [178]. Nach dem ISO 11146:1999-Standard ist das 0. Bildmoment als die Gesamtintensität, das 1. Moment als der Schwerpunkt des Strahls und das 2. Moment als dessen Breite definiert [179]. Analog zu den Momenten in der Statistik entspricht letzteres der Varianz einer Zufallsvariablen und wurde in dem hier gezeigten Experiment zur Bestimmung der Fokusgröße verwendet [162]. Im Ergebnis wurde auf diese Weise eine Auflösung von $374 \pm 30 \mathrm{~nm}$ berechnet und in Abbildung 5.27 miteingezeichnet.

Die Abweichung zu der aus den Fernfeldmessungen erzielten Ergebnissen ist durch die experimentelle Durchführung der „knife-edge“-Messung gegeben. Dadurch, dass nur eines der zwei Schichtpakete der MLL berücksichtigt wurde, ergibt sich nach $R=2 \cdot 0,61 \frac{\lambda f}{b}$ (vgl. Formel 2.28) eine verdoppelte Auflösung. Bei kompletter Bestrahlung der MLL würde sich die Auflösung nach diesem Experiment zu $R=374 / 2=187 \mathrm{~nm}$ abschätzen lassen, welche somit in guter Übereinstimmung mit der Auflösung der Fernfeld-Analyse steht. Experimentelle Beeinträchtigungen sind neben den bereits erläuterten Schwierigkeiten der MLL-Herstellung in einer möglichen unzureichenden Justage sowie einer mangelhaften Oberflächenbeschaffenheit des FeCDrahtes zu sehen. Thermische Instabilitäten der Vakuumschrittmotoren, die für die 


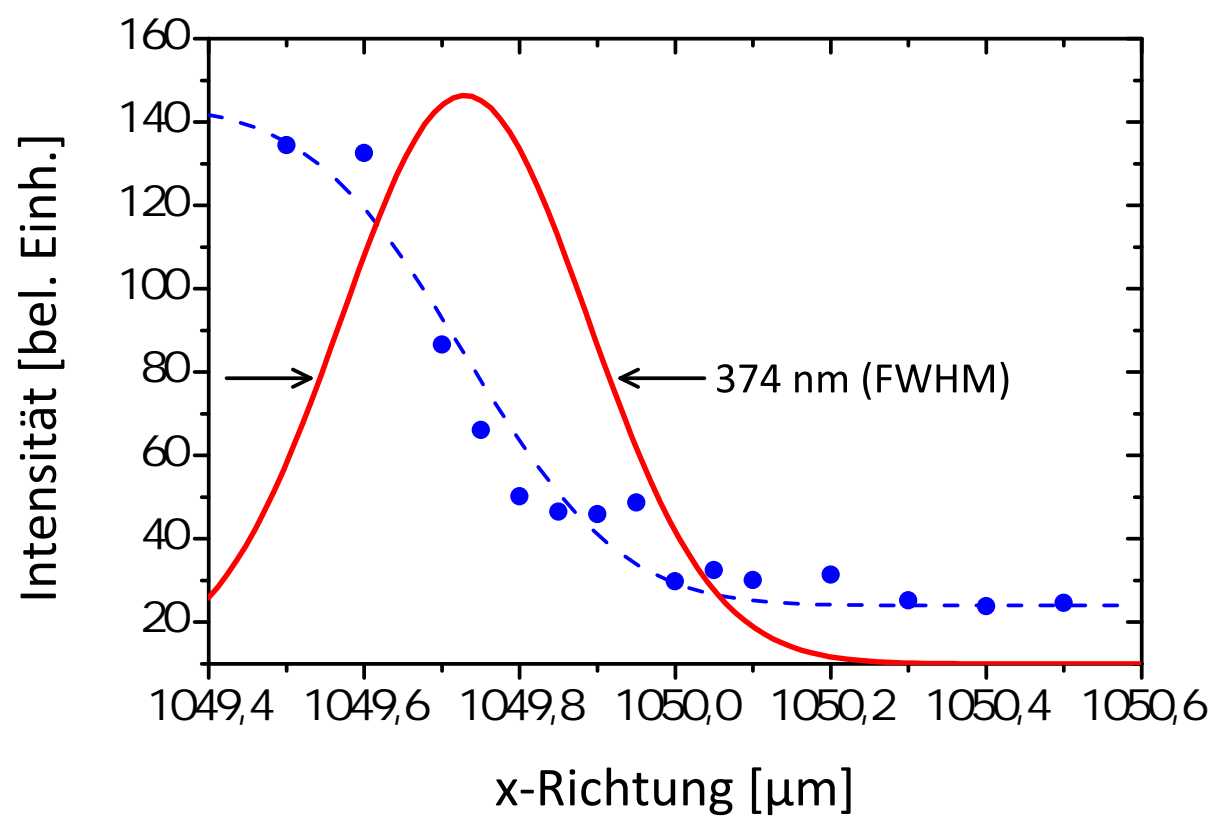

Abbildung 5.27: Intensitätsabfall der 1. Beugungsordnung während einer „knife-edge“-Messung in Abhängigkeit der $z$-Position der Schneidkante senkrecht zur optischen Achse, nahe am Ort des Fokus gemessen. Die Auswertung nach dem 2. Bildmoment führt zu einer Strahlgröße von $374 \pm 30 \mathrm{~nm}$ Halbwertsbreite. (Die Daten wurden freundlicherweise von Michael Reese zur Verfügung gestellt $[157,162]$.)

Bewegung des Drahtes benutzt wurden, könnten ebenfalls einen Einfluss auf die Messung genommen haben.

\subsection{Weitere linear fokussierende Optiken}

Bei der Herstellung der MLL-Teststruktur im vorangegangenen Kapitel haben sich zwei Probleme herauskristallisiert: Zum einen besteht eine unzureichende Konstanz der Depositionsraten, weshalb die Einzelschichtdicken entgegen der geplanten nicht perfekt sind und zum anderen gestaltet es sich als äußerst schwierig, eine präzise Annäherung der MLL-Hälften im FIB durchzuführen.

Zur Stabilisierung der Depositionsraten wurden deshalb vor der Herstellung weiterer Optiken folgende Optimierungsschritte am Herstellungsprozess durchgeführt, die in Kapitel 4 ausführlich erläutert sind: 1. Homogenisierung der ausgegebenen Laserenergie und Verlängerung der Depositionskammer (beides für eine konstante Energiedichte am Target) und 2. Integration einer Targetrotation zur Minimierung der Targetalterung. Ein geringer Abfall der Energiedichte oder der Depositionsrate kann zudem durch eine 
Energie-Korrektur oder eine Erhöhung der Pulszahl im Laserprogramm verhindert werden. Neben diesen Hard- und Software-Optimierungen wurden außerdem die experimentellen Schritte zur Vorbereitung auf eine MLL-Herstellung erweitert, wie sie im nachfolgenden Kapitel erklärt werden.

Folglich der herausgestellten experimentellen Schwierigkeiten bei der Fabrikation einer MLL mittels FIB werden nun Fokussierungsoptiken vorgestellt, die nur aus einer Hälfte einer MLL bestehen. Obwohl sich deren Aufösungen a priori um den Faktor 2 verschlechtern, sind in der Literatur bisher ebenfalls nur „halbe“ MLL bezüglich ihrer Fokussierungseigenschaften untersucht worden. Zwar wurden auch Linsen aus zwei gleichen Schichtpaketen hergestellt [123], deren wahrscheinlich zu geringe Auflösungen aufgrund von Positionierungsfehlern jedoch nicht näher beschrieben.

\subsubsection{Herstellung einer dünnen $\mathrm{MgO} / \mathrm{ZrO}_{2}-\mathrm{MLL}$}

Aufgrund der relativ niedrigen Depositionsrate von Ti und der damit verbundenen hohen benötigten Anzahl an Laserpulsen wurde $\mathrm{MgO}$ als neues Targetmaterial verwendet, dessen optische Eigenschaften bereits anhand Abbildung 5.2 erläutert wurden. Um $\mathrm{MgO}$ in den Herstellungsprozess integrieren zu können, wurden dessen Ablations- und Depositionseigenschaften zunächst einzeln und danach zusammen mit $\mathrm{ZrO}_{2}$ betrachtet. Dabei werden die folgenden Experimente die Notwendigkeit über die genaue Kenntnis der Depositionsraten zeigen. Um einen optimalen Wert zu finden, wurden zunächst mittels des in Kapitel 3.5 vorgestellten Depositionsratenmonitors Schichtdickenmessungen bei unterschiedlichen Linsenpositionen durchgeführt. Bei konstant gehaltener Laserenergie ergibt sich auf diese Weise eine Variation der Energiedichte $\rho=E / A$ (vgl. vorbereitende Ti-Ratenmessung in Abb. 5.5). In Abbildung 5.28 zeigt sich schließlich ein Maximum der Rate mit 0,0093 nm/Puls bei ca. 2,6 cm defokussierter Linsenstellung, welche ungefähr doppelt so hoch wie die des Ti ist. Diese Position wurde für den weiteren experimentellen Verlauf sämtlicher Schichtherstellungen als neuer Standardparameter definiert und entspricht mit einer Laserenergie von $E=70 \mathrm{~mJ}$ einer eingekoppelten Energiedichte von $\rho=1,8 \mathrm{~J} / \mathrm{cm}^{2}$.

Wie stabil die Gesamtdepositionsrate von $\mathrm{MgO} / \mathrm{ZrO}_{2}-$ Multischichten aufgrund der eingangs erwähnten Optimierungen über einen längeren Zeitraum gehalten werden kann, ist in Abbildung 5.29 anhand eines Schichtdickenverlaufs eines periodischen Multischichtpakets mit insgesamt 64Doppelschichten einer jeweiligen Einzelschichtdicke von $10 \mathrm{~nm}$ dargestellt. Dabei deutet die lineare Anpassung des gemittelten 


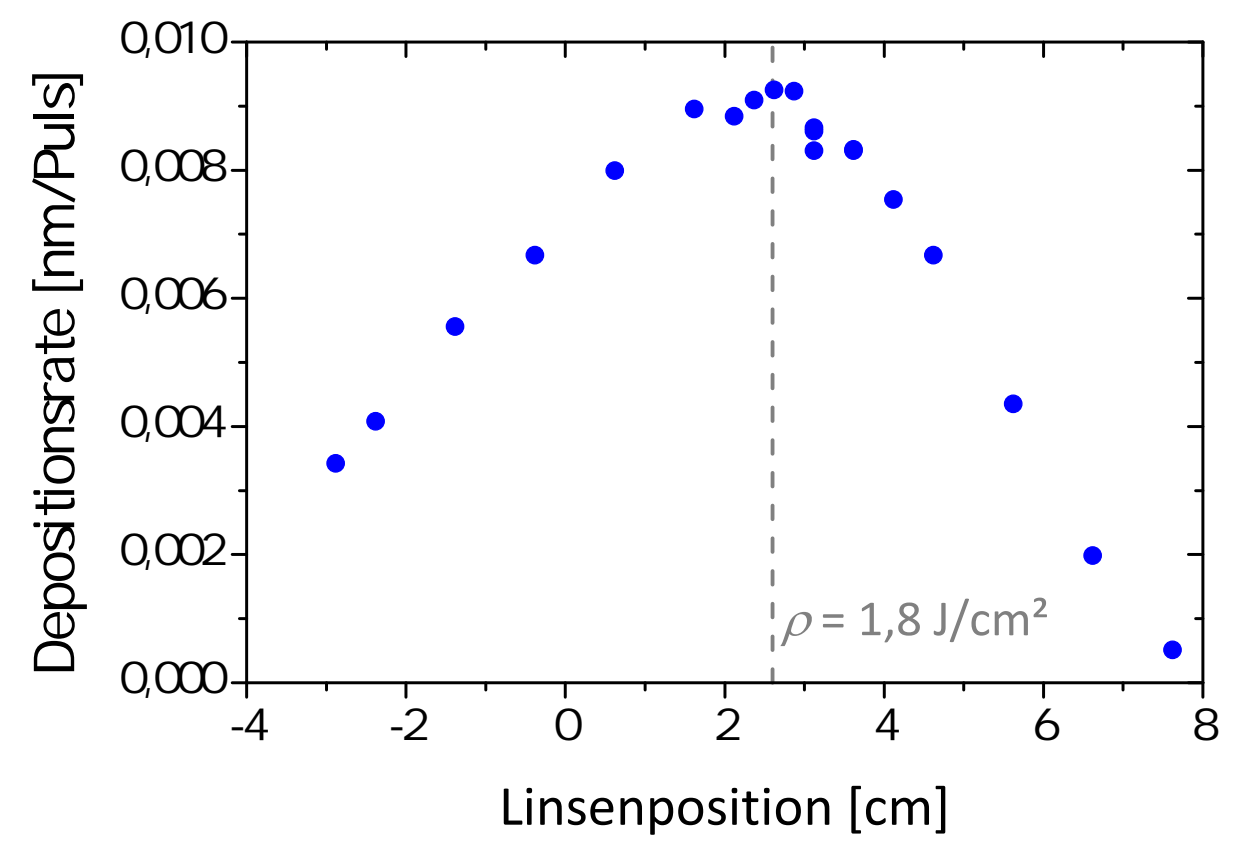

Abbildung 5.28: Depositionsrate von $\mathrm{MgO}$ in Abhängigkeit der Strahlfokussierung bei unterschiedlichen Linsenpositionen. Die maximale Depositionsrate von 0,0093 nm/Puls stellt sich bei einer Linsenposition von $2,6 \mathrm{~cm}$ ein.

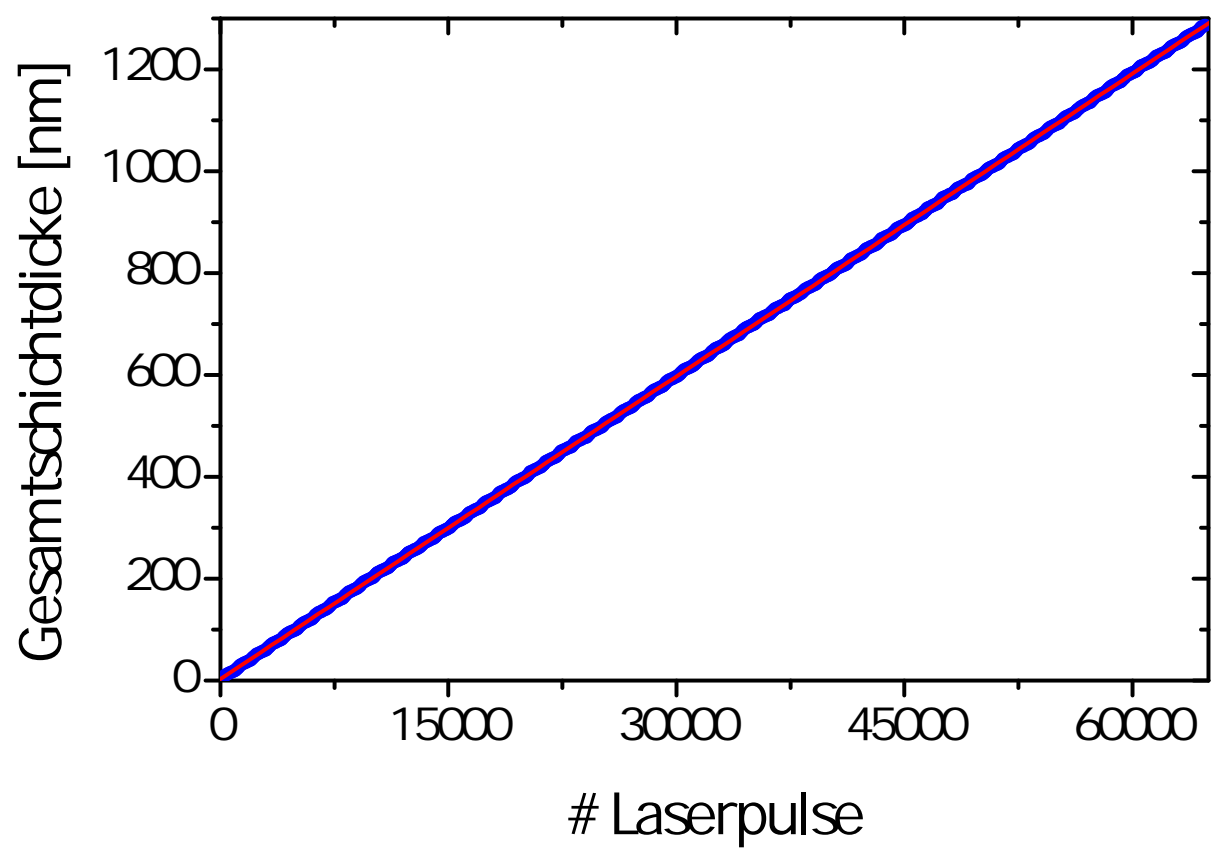

Abbildung 5.29: Deposition eines $64 \mathrm{x}(10 \mathrm{~nm} / 10 \mathrm{~nm}) \mathrm{MgO} / \mathrm{ZrO}_{2}-$ Schichtsystems, dargestellt als Funktion der abgegebenen Laserpulse. Selbst bis zu einer Gesamtschichtdicke von 1,28 $\mu \mathrm{m}$ zeigt sich eine sehr genaue Konstanz der Depositionsraten. 
Anstiegs der Schichtdicke der beiden Einzelkomponenten bei einer Gesamtschichtdicke von knapp 1,3 $\mu$ m auf eine hohe Konstanz der Depositionsraten von $\mathrm{ZrO}_{2}$ und $\mathrm{MgO}$ hin. Wird das Schichtwachstum an den Grenzflächen betrachtet (s. Abb. 5.30), zeigt sich außerdem keine Veränderung der Dickenzunahme, sodass beim Materialsystem $\mathrm{MgO} / \mathrm{ZrO}_{2}$ unter den gewählten Herstellungsbedingungen kein Fremdsputteryield berücksichtigt werden muss.
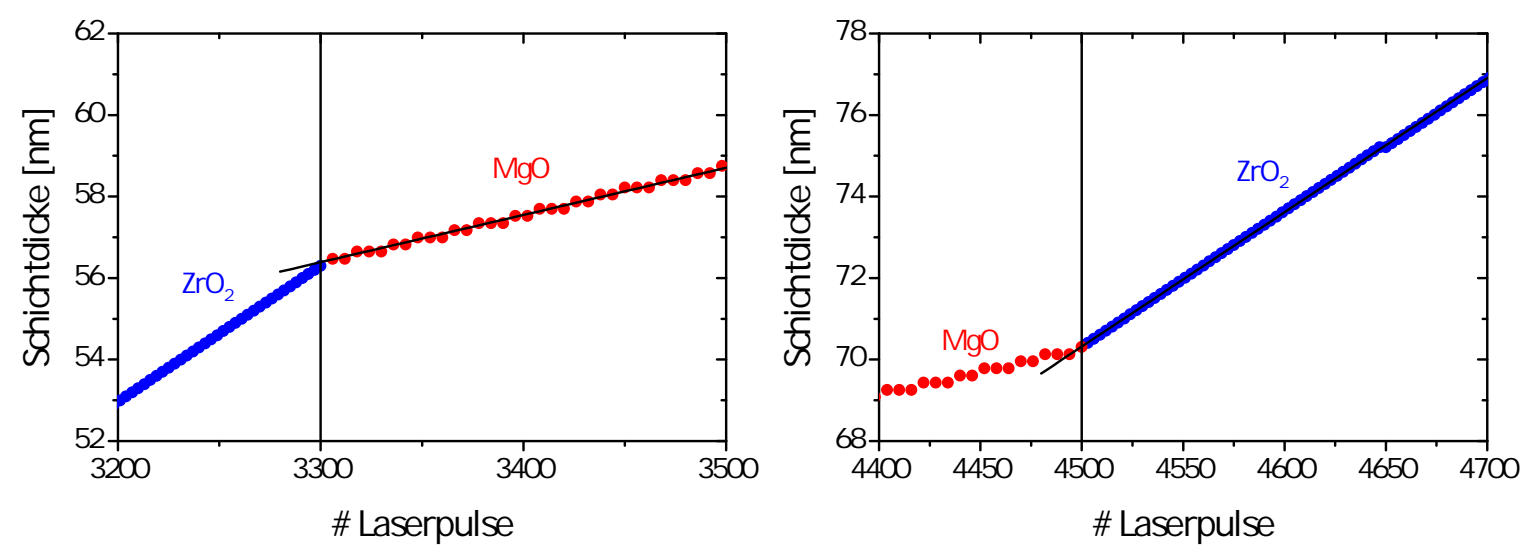

Abbildung 5.30: Detaillierte Verläufe der deponierten Schichtdicke von $\mathrm{ZrO}_{2}$ und $\mathrm{MgO}$ an den Grenzflächen. Durch den jeweils herrschenden linearen Anstieg beim Materialwechsel lässt sich ein Wiederabtrag an den Grenzflächen ausschließen.

Obwohl sich mit Hilfe des Depositionsratenmonitors nach dem Prinzip der Massenwägung sehr genaue Änderungen der Schichtdicke messen lassen, sind absolute Angaben hingegen abhängig von der Eichung des Gerätes. Zur Vorbereitung für eine MLLHerstellung wurde daher neben den obigen gewonnenen Erkenntnissen die Schichtdickenanalyse mittels Röntgenreflektometrie hinzugezogen. Durch entsprechende Simulationen konnten die Schichtdicken und insbesondere die Depositionsraten sehr präzise bestimmt werden. In Abbildung 5.31 ist zunächst der Verlauf der XRR-Spektren von $\mathrm{ZrO}_{2}-$ und $\mathrm{MgO}-$ Einzelschichten mit geplanten Schichtdicken von je $50 \mathrm{~nm}$ dargestellt. Die jeweiligen Schichtdicken konnten aus den Simulationen $\mathrm{zu} d_{\mathrm{ZrO}_{2}}=51,6 \mathrm{~nm}$ und $d_{\mathrm{MgO}}=44,5 \mathrm{~nm}$ bestimmt werden, woraus sich neu gewonnene Depositionsraten von 0,0337 nm/Puls für $\mathrm{ZrO}_{2}$ und 0,0127 nm/Puls für $\mathrm{MgO}$ ergeben.

Anhand periodischer $\mathrm{ZrO}_{2} / \mathrm{MgO}-$ Multischichten wurde dann eine sehr sensitive Bestimmung der Depositionsraten von $\mathrm{ZrO}_{2}$ und $\mathrm{MgO}$ vorgenommen, indem mittels verschiedener Proben ein genaues Schichtdickenverhältnis von $\Gamma=0,5$ gesucht wurde. Dies ist genau dann der Fall, wenn die geraden Bragg-Peaks der XRRSpektren ausgelöscht bzw. aufgrund vorhandener Rauigkeiten minimiert werden [146]. In Abbildung 5.32 sind dazu XRR-Messungen zweier Multischichten mit geplanten 


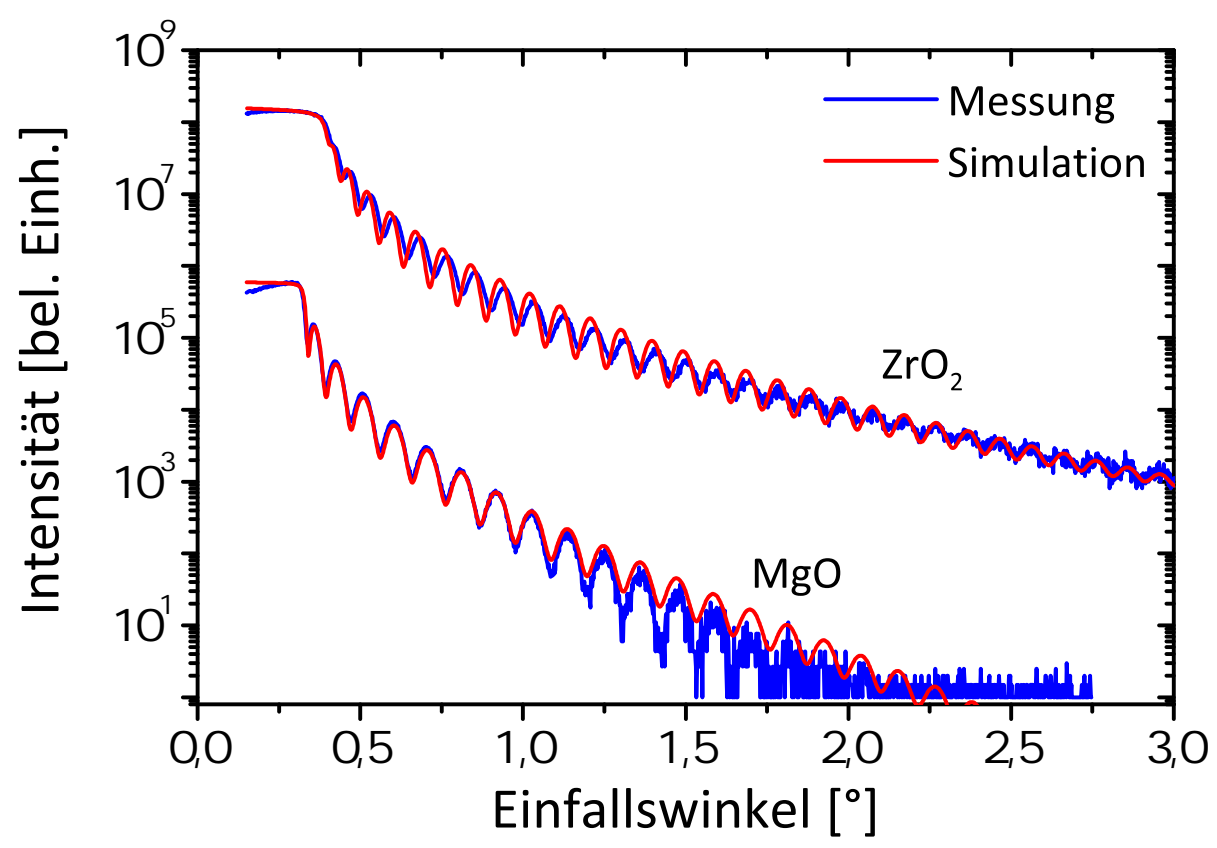

Abbildung 5.31: XRR-Messungen und Simulationen von $\mathrm{ZrO}_{2}-$ und $\mathrm{MgO}$-Einzelschichten zur detaillierten Bestimmung der einzelnen Depositionsraten. Aufgrund einer Hydroxid-Bildung an der MgO-Schichtoberfläche kommt es zu einer erhöhten Rauigkeit von $\sigma_{\mathrm{MgO}}=0,6 \mathrm{~nm}$, sichtbar durch den stärkeren Intensitätsabfall. Zur besseren Übersicht sind die einzelnen Messkurven intensitätsverschoben.

Einzelschichtdicken von $d=10 \mathrm{~nm}$ und 20 Doppelschichten mit unterschiedlichem Dickenverhältnis gezeigt. Bei dem unteren Intensitätsverlauf sind die Depositionsraten noch nicht optimal gewählt worden, sodass sich mit $\Gamma=0,535$ die Einzelschichtdicken $\mathrm{zu} d_{\mathrm{ZrO}_{2}}=10,6 \mathrm{~nm}$ und $d_{\mathrm{MgO}}=10,4 \mathrm{~nm}$ ergeben. Mit korrigierten Depositionsraten konnte eine weitere Multischicht mit den exakten Vorgaben von $10 \mathrm{~nm}$ Einzelschichtdicke und $\Gamma=0,5$ hergestellt werden. Die zugehörige XRR-Analyse (Abb. 5.32 oben) weist schwach ausgeprägte gerade Bragg-Peaks auf. Die hohe Anzahl sichtbarer Reflexe bis zu großen Einfallswinkeln spricht für eine hochpräzise Schichtherstellung mit hervorragender Grenzflächenschärfe. Die genauen Depositionsraten für die MLLHerstellung belaufen sich nach dieser Messung $\mathrm{zu} 0,0326 \mathrm{~nm} / \mathrm{Puls}$ für $\mathrm{ZrO}_{2}$ und 0,0141 nm/Puls für MgO.

Das Design der herzustellenden MLL wurde ähnlich wie in Kapitel 5.1.1 nach der weichen Röntgenwellenlänge $\lambda=2,879 \mathrm{~nm}$ und einer Fokuslänge von $f=100 \mu \mathrm{m}$ gewählt. Mit einer theoretischen Gesamtschichtdicke von $1005 \mathrm{~nm}$ wurden nach dem Zonenplattenbildungsgesetz die 624. - 720. Zone mit Schichtdicken von 10,74-10,00 nm mit einer Gesamtpulszahl von knapp 50000 laserdeponiert, wobei mit der dünnsten 


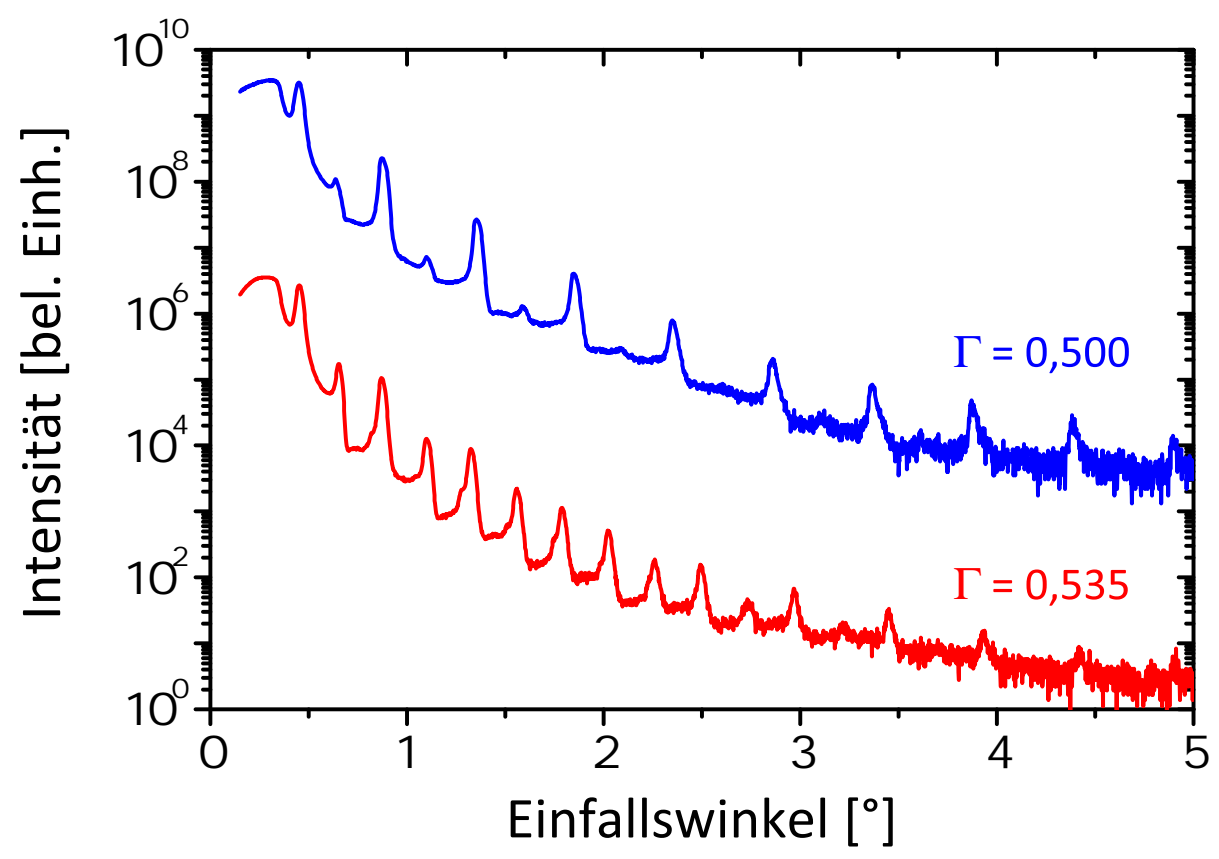

Abbildung 5.32: Präzise Bestimmung der Depositionsraten von $\mathrm{ZrO}_{2}$ und $\mathrm{MgO}$ mit Hilfe der Herstellung periodischer Multischichten. Durch ein Schichtdickenverhältnis von $\Gamma=0,5$ ergibt sich eine Minimierung der geraden Bragg-Peaks, wodurch eine sehr sensitive Bestimmung der Raten zugänglich wird.

Schicht aus $\mathrm{ZrO}_{2}$ begonnen wurde. Während der Herstellung wurde zum ersten Mal mit einer linearen Energiekorrektur von $3,4 \cdot 10^{-5} \mathrm{~mJ} / \mathrm{Puls}$ gearbeitet, um den durch die Verschmutzung der Eintrittsscheibe der Vakuumkammer entstehenden Energieverlust zu kompensieren. Ein REM-Querschnitt des resultierenden $\mathrm{MgO} / \mathrm{ZrO}_{2}$-Schichtpakets ist in Abbildung 5.33 gezeigt, welches in dieser Materialkombination ebenfalls keine Entstehung einer kumulativen Rauigkeit erkennen lässt. Mit einer gemessenen Gesamtschichtdicke von $987 \mathrm{~nm}$ im FIB ergibt sich eine Abweichung zur Soll-Schichtdicke von nur 1,8\%. Eine solch präzise Realisierung einer vorgegebenen Schichtdicke wäre ohne die aufwändigen experimentellen Vorbereitungen nicht möglich gewesen.

Die Fabrikation der MLL im FIB vollzog sich auf gleiche Weise wie in Kapitel 5.1.4 und wird hier nicht näher erläutert. Diesmal wurde allerdings wie eingangs erwähnt nur ein Schichtpaket im Querschnitt als MLL benutzt, sodass ein kompliziertes Zusammenfügen zweier Lamellen ausbleibt. In Abbildung 5.34 oben ist die auf ca. $200 \mathrm{~nm}$ fertig ausgedünnte MLL auf dem $\mathrm{Cu}$-Probenhalter dargestellt. Die Linse wurde anschließend auf einem Fenster eines $\mathrm{Si}_{3} \mathrm{~N}_{4}$-Substrats zur Generierung eines äußeren beam stops fixiert (s. Abb. 5.34 unten). 


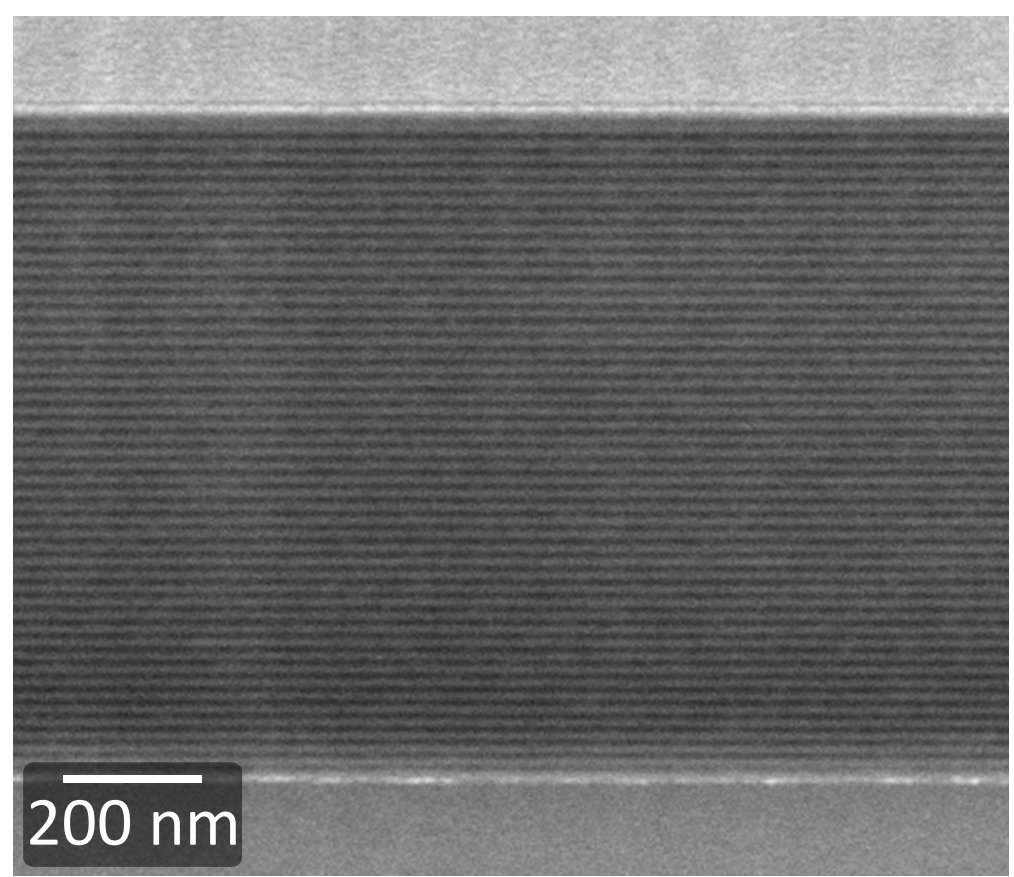

Abbildung 5.33: REM-Querschnittsaufnahme der hergestellten aperiodischen $\mathrm{MgO} / \mathrm{ZrO}_{2}-$ Multischicht mit einer prozentualen Abweichung von lediglich 1,8\% zur Soll-Schichtdicke. Auch bei diesem Materialsystem zeichnet sich keine kumulative Rauigkeit aus.

Durch das von Michael Reese durchgeführte Divergenzwinkel-Experiment an dieser MLL konnte anhand der Abstände von 1. und 0. Beugungsordnung analog zu Abbildung 5.25 die Fokuslänge zu $f=98 \mu \mathrm{m}$ bestimmt werden [162], welche in guter Übereinstimmung mit dem Designparameter steht $(f=100 \mu \mathrm{m})$. Leider konnte aufgrund zu niedriger Intensität am Ort des Fokus als Folge der geringen Aperturgröße von $20 \mu \mathrm{m}^{2}$ keine „knife-edge“-Messung durchgeführt werden, um die Fokussierungseigenschaften der MLL zu bestimmen. 

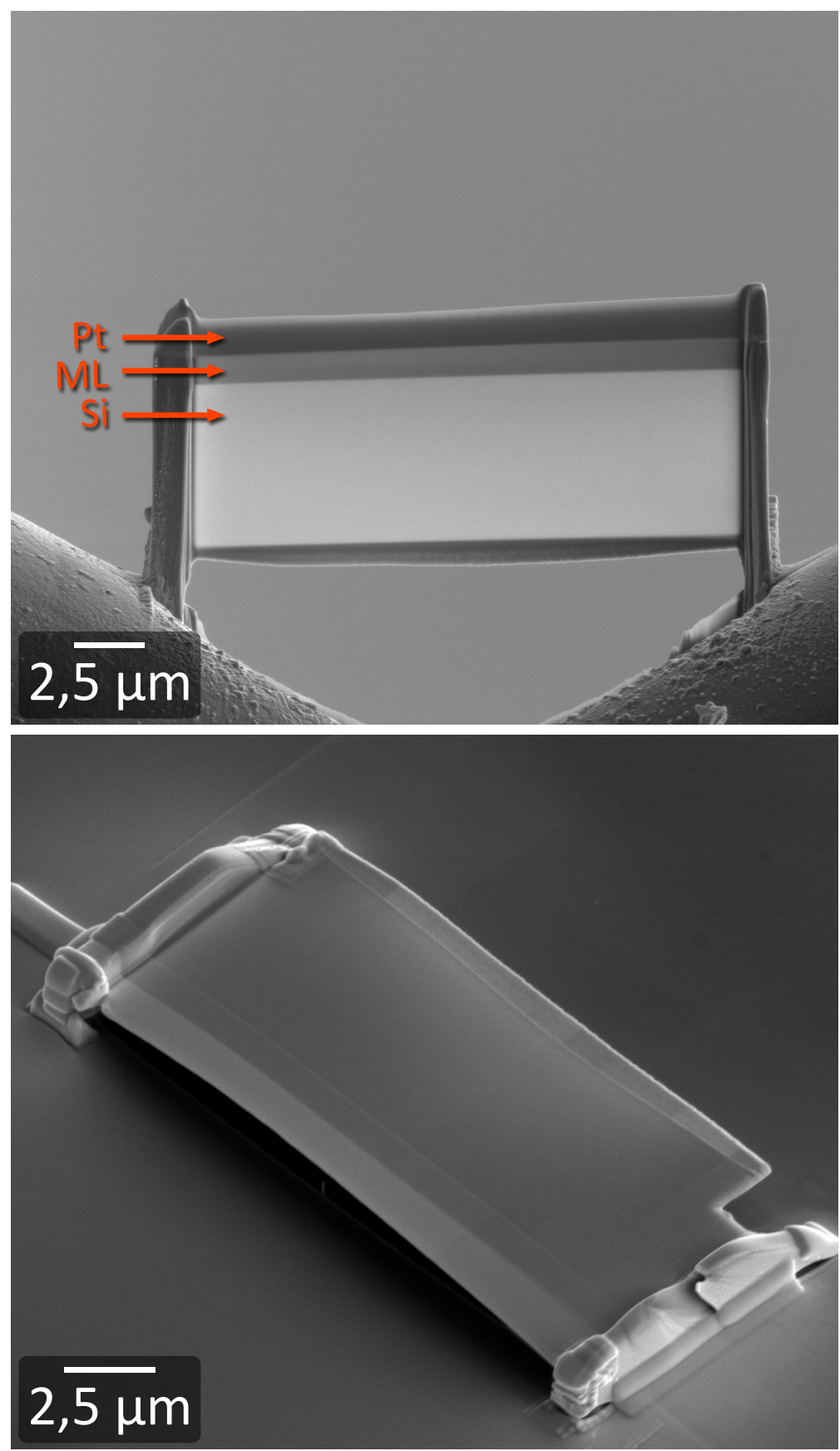

Abbildung 5.34: Fabrikation der MLL im FIB nach bekanntem Schema (vgl. Kap. 5.1.4): Nach der Ausdünnung auf ca. $200 \mathrm{~nm}$ (oben) wird das Schichtpaket auf ein zuvor geschnittenes Fenster in einem $\mathrm{Au}$-bedampften $\mathrm{Si}_{3} \mathrm{~N}_{4}$-Substrat gelegt (unten). 


\subsubsection{Herstellung einer dicken $\mathrm{MgO} / \mathrm{ZrO}_{2}-\mathrm{MLL}$}

Um eine höhere Intensität zu erlangen, wurde im Folgenden die Fabrikation einer wesentlich dickeren MLL mit einer geplanten Gesamtschichtdicke von 9,6 $\mu \mathrm{m}$ nach den gleichen Designvorgaben getestet. Bisher war überhaupt nicht klar, ob sich sehr dicke Schichten ohne ein Abplatzen vom Substrat laserdeponieren lassen und ob die Multischichtstruktur im FIB auf eine gleichmäßige optische Tiefe von nur $200 \mathrm{~nm}$ ausgedünnt werden kann. Die Schichtdicken der 641 deponierten Zonen reichten in diesem Experiment von $30-10 \mathrm{~nm}$ und sind in der Übersicht in Abbildung 5.35 dargestellt. Obwohl nur die dickeren Schichten klar voneinander getrennt zu erkennen sind, lässt sich auch hier über die gesamte gemessene Dicke von ca. 9,3 $\mu \mathrm{m}$ keine Entstehung einer kumulativen Rauigkeit festhalten. Die 3\%-ige Abweichung von der Soll-Schichtdicke macht sich besonders bei den dickeren, später hergestellten Zonen bemerkbar, die im Durchschnitt knapp $3 \mathrm{~nm}$ zu dünn ausgefallen sind. Nach dem gleichmäßigen Ausdünnungsprozess auf $200 \mathrm{~nm}$ optische Tiefe kam ein großer Droplet zum Vorschein, der eine Störung der Schichtstruktur entsprechend des Zonenplattenbildungsgesetzes hervorruft. Diese wurde im Laufe weiterer Multischichtdeposition durch Glättung mit Hilfe von $\mathrm{ZrO}_{2}$ behoben.

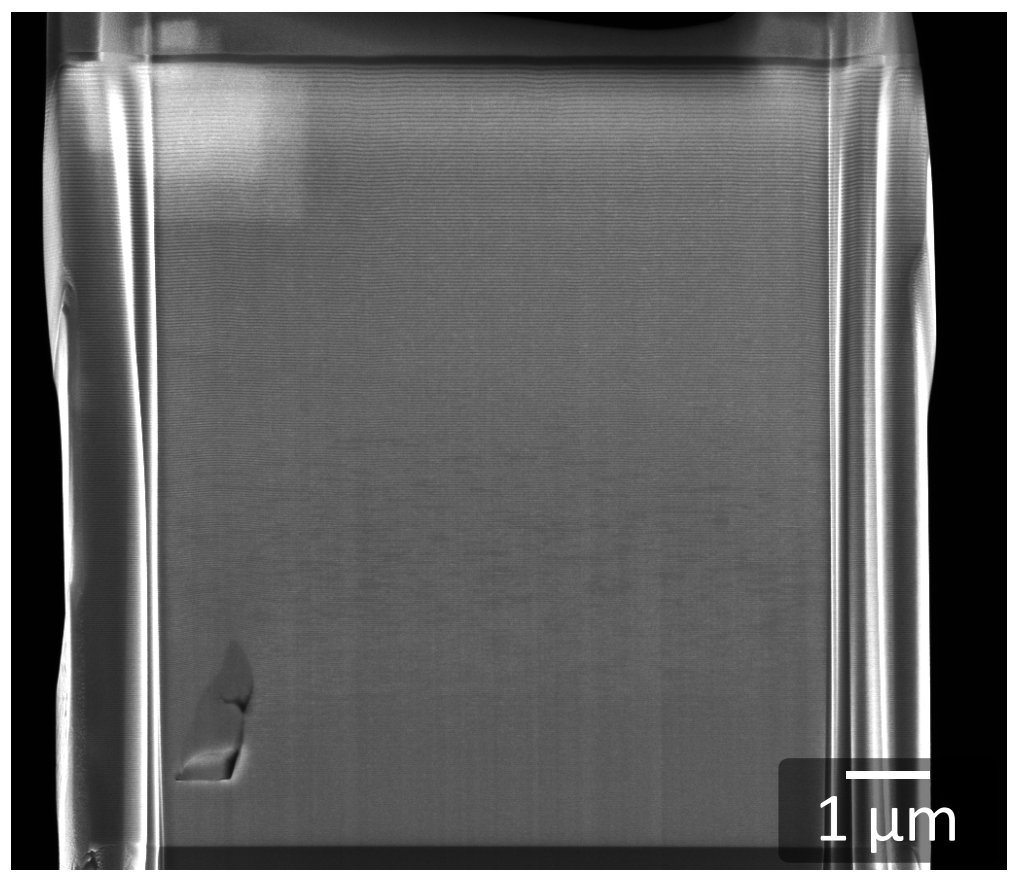

Abbildung 5.35: REM-Querschnitt einer $9,3 \mu \mathrm{m}$ dicken $\mathrm{MgO} / \mathrm{ZrO}_{2}-\mathrm{MLL}$. Trotz der enormen Gesamtschichtdicke scheint eine ausreichende Haftung zu bestehen, sodass die Multischicht nicht abreißt. Ferner kann auch hier keine kumulative Rauigkeit festgestellt werden. 
In Abbildung 5.36 ist schließlich der Transfer der MLL auf ein $\mathrm{Si}_{3} \mathrm{~N}_{4}$-Substrat gezeigt. Die Ausmaße des im FIB geschnittenen Fensters sind so gewählt worden, dass der gestörte Bereich der Multischicht auf dem $\mathrm{Si}_{3} \mathrm{~N}_{4}$ aufliegt und somit von der Beleuchtung mit Röntgenstrahlung ausgenommen ist. Weiterhin können eventuelle Störungen der Schichtstruktur durch eine gezielte Pt-Deposition mittels FIB abgedeckt werden, wie es in Abbildung 5.36 rechts demonstriert wurde. Insgesamt hat sich bei dieser MLL-Teststruktur gezeigt, dass sehr dicke Multischichten ohne ein Abplatzen vom Substrat mittels PLD hergestellt werden können. Eine Untersuchung der fokussierenden Eigenschaften der MLL ist bislang noch nicht erfolgt, aber für zukünftige Messungen in Planung. Bei einer hohen Intensität sollte die Linse mit Sicherheit eine Auflösung im Bereich von $50 \mathrm{~nm}$ besitzen.
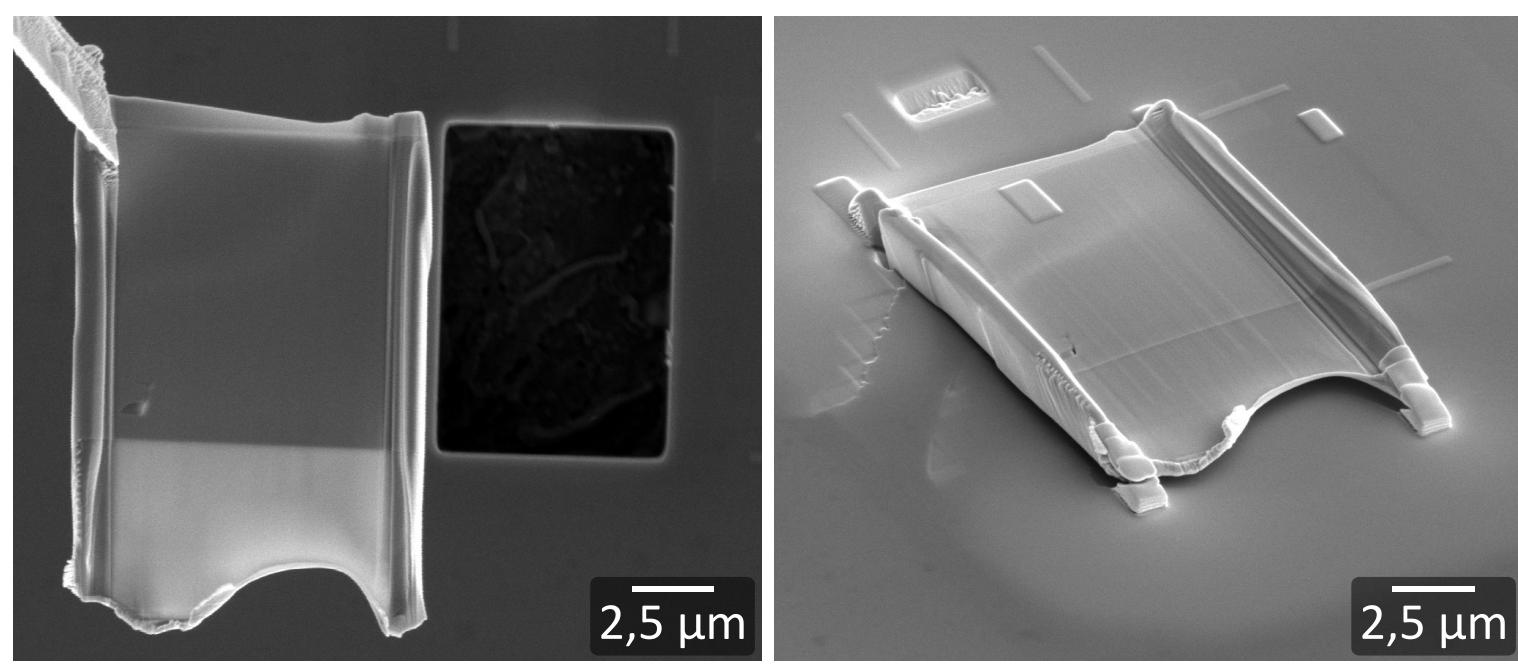

Abbildung 5.36: FIB-Transfer der MLL auf ein zuvor geschnittenes Loch in einem $\mathrm{Si}_{3} \mathrm{~N}_{4}-$ Substrat. Die Linse wurde so über dem Fenster positioniert, dass der gestörte Bereich auf dem Substrat aufliegt und somit nicht zu der Fokussierung beiträgt.

\subsubsection{Herstellung eines $\mathrm{Ti} / \mathrm{ZrO}_{2}-$ Röntgenmonochromators}

Abschließend wird eine weitere MLL-Teststruktur vorgestellt, die aus einer noch dickeren Multischicht als im vorigen Abschnitt fabriziert wurde und durch spezielle Arbeitsschritte im FIB als Monochromator eingesetzt werden kann. Dazu wird wie in Abbildung 5.37 schematisch gezeigt eine partielle MLL in Verbindung mit einer ordnungsselektierenden Lochblende (engl. order sorting aperture, OSA) benutzt, die mit einer sehr schmalen Öffnung im Abstand der Fokuslänge der MLL positioniert wird. Da sich die Fokuslänge nach dem Zonenplattenbildungsgesetz mit variierender 


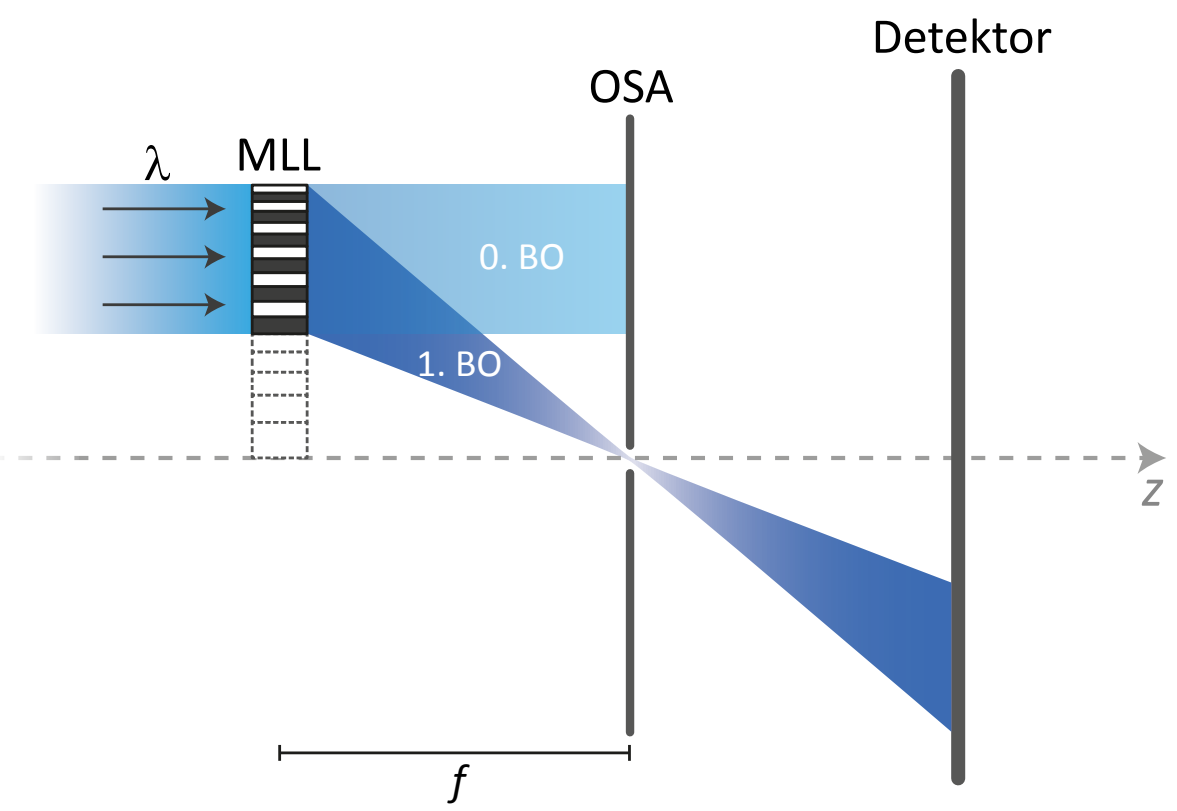

Abbildung 5.37: Schematische Darstellung eines Röntgenmonochromators. Wird im Abstand der Brennweite der Linse eine ordnungsselektierende Aperturblende (OSA) mit einem kleinen Loch positioniert, kann nur monochromatische Röntgenstrahlung 1. Beugungsordnung die Lochblende passieren und zur mikroskopischen Abbildung benutzt werden. (Zur Übersichtlichkeit ist die MLL gegenüber der Lochblende und dem Detektor stark vergrößert dargestellt.)

Wellenlänge ändert, kann nur monochromatische Strahlung 1. Beugungsordnung durch die Blendenöffnung hindurch treten, um eine dort befindliche Probe zu beleuchten. Der Vorteil einer solchen kombinierten Optik ist in der erleichterten Justage im Strahlengang eines Röntgenmikroskops zu sehen, da Linse und Blende fest miteinander verbunden sind. Hinsichtlich dieser Geometrie wurde getestet, ob sich eine MLL auf einen im FIB deponierten Sockel über einer Lochblende platzieren lässt.

Wiederum wurde nach dem Zonenplattenbildungsgesetz für eine Wellenlänge von $\lambda=2,879 \mathrm{~nm}$ ein aperiodisches $\mathrm{Ti} / \mathrm{ZrO}_{2}$-Schichtpaket mit 285 Zonen hergestellt, deren Schichtdicken von $80-24 \mathrm{~nm}$ reichen. Mit einer Gesamtschichtdicke von ca. $17 \mu \mathrm{m}$ ist diese off-axis-MLL die dickste im Rahmen dieser Arbeit hergestellte Teststruktur. Der Querschnitt in Abbildung 5.38 lässt über die gesamte Schichtstruktur keinerlei Entstehung einer kumulativen Rauigkeit vermuten. Auch mechanische Verspannungen im Film scheinen klein genug zu sein, sodass keine Haftungsprobleme zur Substratoberfläche (Abb. 5.38 unten) entstehen. Somit zeigt sich, dass mit PLD die Deposition sehr vieler Multischichten mit einer konstant hohen Qualität möglich ist.

Bei der Präparation der MLL im FIB konnte selbst bei einer so großen Gesamtschichtdicke eine gleichmäßige Ausdünnung auf $200 \mathrm{~nm}$ optische Tiefe erreicht werden. 


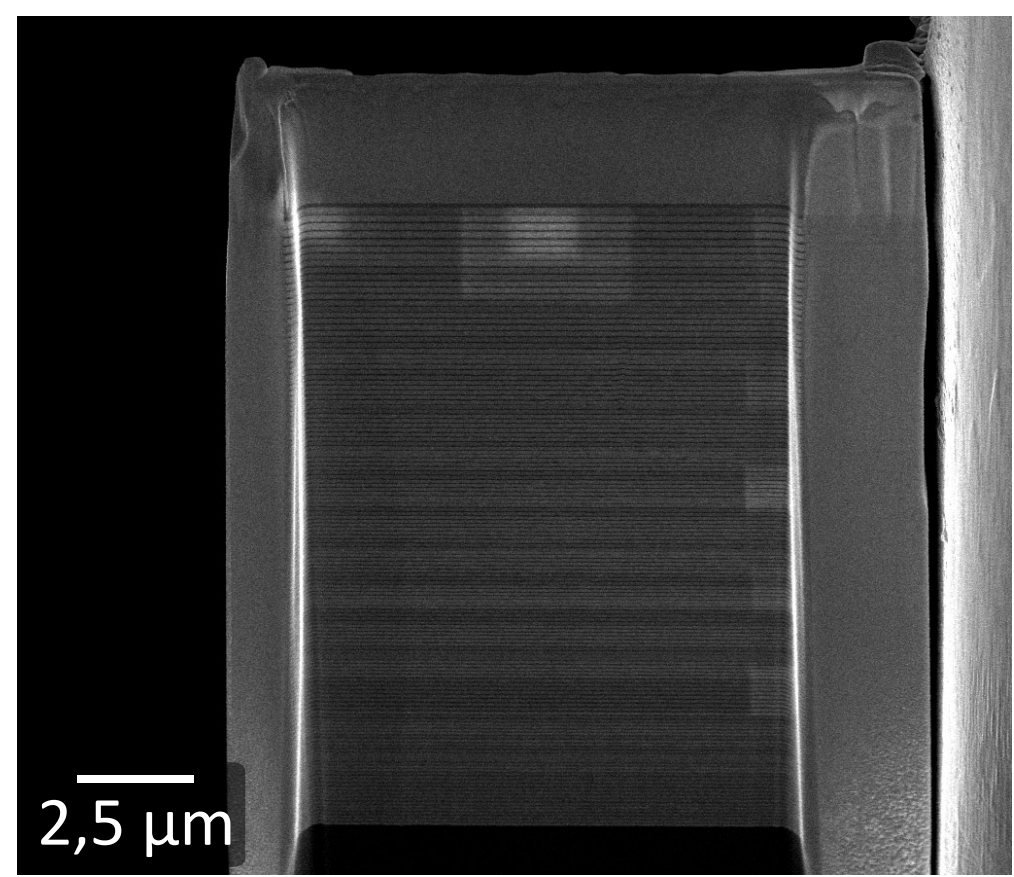

Abbildung 5.38: REM-Querschnittsaufnahme der im Rahmen dieser Arbeit bislang größten hergestellten MLL-Teststruktur. Trotz der enormen Gesamtschichtdicke von ca. $17 \mu \mathrm{m}$ weist die Multischicht eine ausreichende Haftung zum Substrat auf. Weiterhin deutet sich keine Entstehung einer kumulativen Rauigkeit an [176].

Im nächsten Schritt wurde mit dem FIB ein rechteckiges Loch in den gedünnten Bereich des $\mathrm{Si}_{3} \mathrm{~N}_{4}$-Substrats geschnitten sowie mittels Pt-Deposition vier Säulen erstellt, auf die die MLL platziert wurde (Abb. 5.39). Die Höhe der Säulen entspricht in diesem TestExperiment nicht exakt der Länge der Brennweite, sondern sind zur Demonstration ungefähr $3 \mu \mathrm{m}$ hoch deponiert worden. Im rechten Teil der Abbildung 5.39 wurde die MLL bereits mit den Säulen kontaktiert und mittels weiterer Pt-Deposition fixiert, sodass die Omniprobe-Spitze (oben links im Bild) abgetrennt werden konnte. Das Ergebnis in Abbildung 5.40 zeigt schließlich die mit der Lochblende fest verbundene MLL als Demonstrator zur Monochromatisierung von Röntgenstrahlung. 

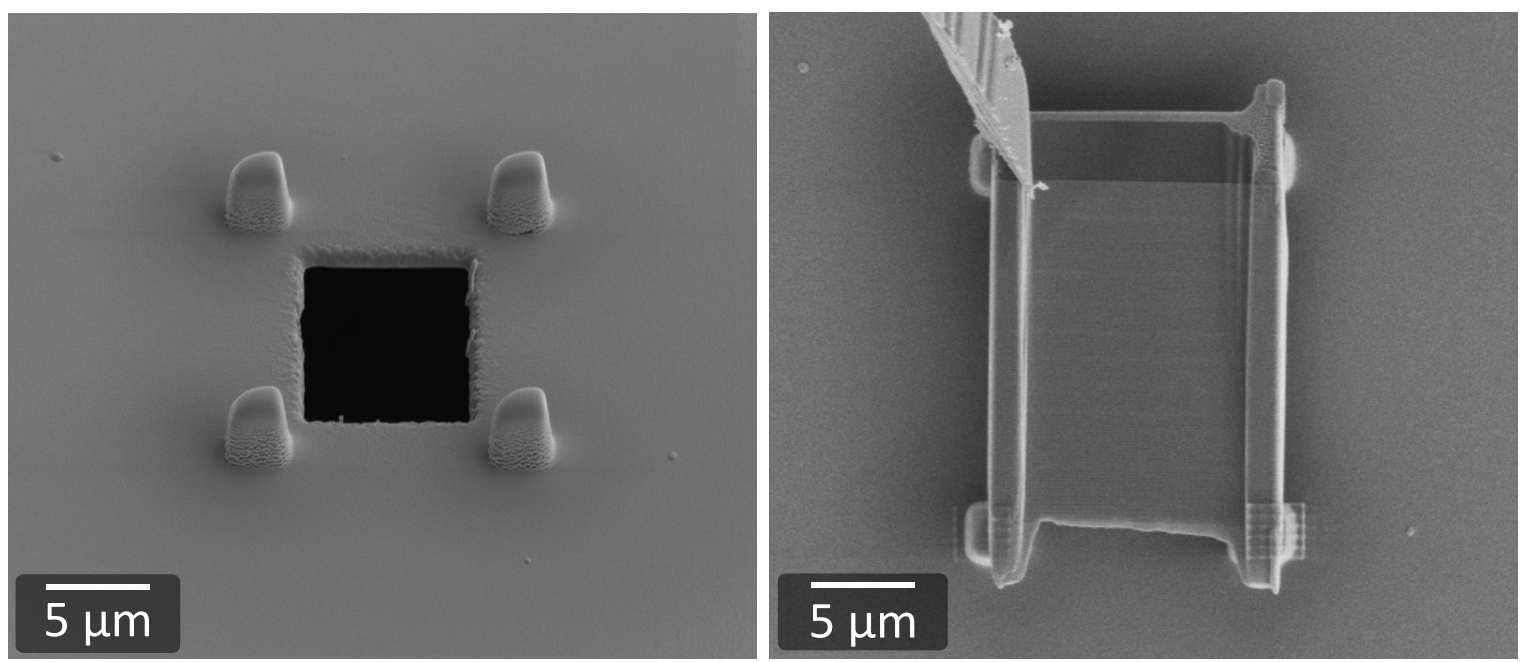

Abbildung 5.39: Benötigte FIB-Arbeitsschritte zur Realisierung eines Röntgenmonochromators. Um ein rechteckig geschnittenes Loch in einem $\mathrm{Si}_{3} \mathrm{~N}_{4}$-Substrat herum wurden $3 \mu \mathrm{m}$ hohe Pt-Säulen ionenstrahldeponiert, auf die die MLL mittels der Omniprobe aufgesetzt und mit weiterer PtDeposition fixiert wurde.

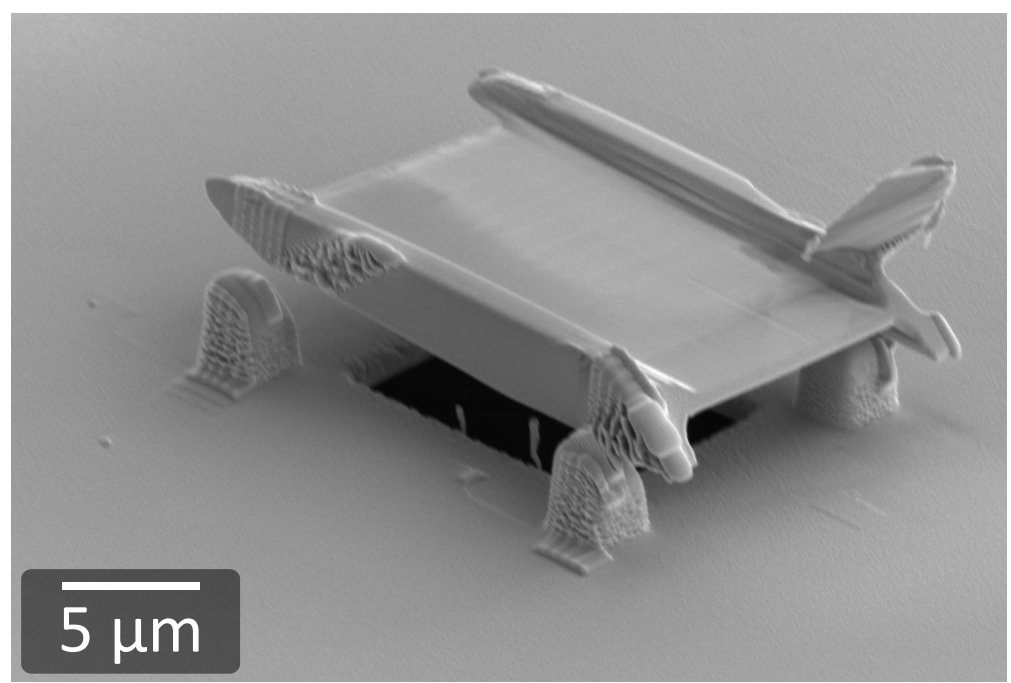

Abbildung 5.40: Fertig gestellter Demonstrator eines Röntgenmonochromators mittels FIB. Die feste Verbindung zweier optischer Elemente (MLL und Aperturblende) verringert aufwändige Justagearbeiten bei der Verwendung eines fokussierenden Monochromators in der Röntgenmikroskopie [176]. 


\subsection{Laserdeponierte Multilagen-Zonenplatten}

Da sich mit allen bisher aufgezeigten Transmissionsoptiken aufgrund der planaren Schichtstruktur ausschließlich Linienfoki erzeugen lassen, sollen im Folgenden Demonstratoren laserdeponierter Multilagen-Zonenplatten (MZP) zur Generierung eines zweidimensionalen Punktfokus vorgestellt werden. Dafür muss ein Multischichtsystem auf einen Draht deponiert werden, um die notwendige konzentrische Zonengeometrie zu erzeugen. Die Vorteile einer MZP gegenüber lithographisch hergestellten konventionellen Zonenplatten liegen in der Einstellbarkeit beliebig hoher Aspektverhältnisse bei kleinsten äußeren Zonenbreiten, wie sie für eine effiziente Fokussierung von Röntgenstrahlung benötigt wird [50]. Nachteilig tritt allerdings bei der Beschichtung einer gekrümmten Oberfläche die begünstigte Entstehung kumulativer Rauigkeiten aufgrund von Abschattungseffekten in den Vordergrund [114]. Hinsichtlich der erreichbaren Fokusgröße stellt diese Art von Rauigkeit den limitierenden Faktor bei den in der Literatur veröffentlichten Untersuchungen an gesputterten MZP (s. Kap. 2.3) dar. Kumulative Rauigkeiten sind bei dieser Herstellungsmethode neben dem Materialsystem oder den Herstellungsparametern [116] außerdem vom Durchmesser des zu beschichtenden Drahtes abhängig [117]. Für die Herstellung laserdeponierter MZP ist daher durch mehrere Vorexperimente die Prüfung notwendig, ob die Beschichtung gekrümmter Oberflächen überhaupt ohne Entstehung kumulativer Rauigkeiten möglich ist, welche die äußere und somit aufösungsbestimmenden Schichtstrukturen maßgeblich in ihrer Qualität beeinflussen würden. Des Weiteren muss eine ausreichende Haftung zwischen Multischicht und Substrat bestehen, was im Folgenden überprüft werden soll.

\subsubsection{Vorbereitende Beschichtungsprozesse}

Bei einer MLL-Schichtherstellung wurde bisher aufgrund der frei wählbaren ZonenReihenfolge mit der Herstellung der dünnsten Schichten begonnen, um deren Positionierung so genau wie möglich einzuhalten. Für die Herstellung einer MZP muss der Radius der Zonen nach dem Zonenplattenbildungsgesetz allerdings nach außen hin abnehmen, sodass hier mit der Deposition der dicksten Schicht gestartet werden muss. Ob diese „bottom-up“-Deposition die Entstehung einer kumulativen Rauigkeiten begünstigt, ist in Abbildung 5.41 zunächst mit der Herstellung einer planaren MLLStruktur erprobt worden. Dabei wurden mit vorgegebener Wellenlänge $(\lambda=2,879 \mathrm{~nm})$ und einer verkürzten Fokuslänge von $f=25 \mu \mathrm{m} 196 \mathrm{Ti}-$ und $\mathrm{ZrO}_{2}$-Einzelschichten 
einer vollen Zonenplattenstruktur mit Schichtdicken von ca. 270-13 nm hergestellt. Bei der Betrachtung der REM-Übersichtsaufnahme sowie der detaillierten Vergrößerung der dünnsten Schichten in Abbildung 5.41 rechts lässt sich über den gesamten Bereich der 5,2 $\mu \mathrm{m}$ dicken Multischicht keine Entstehung einer additiven Rauigkeit erkennen. Die Depositionsreihenfolge der $\mathrm{Ti} / \mathrm{ZrO}_{2}-$ Multischichten spielt demnach keine Rolle.

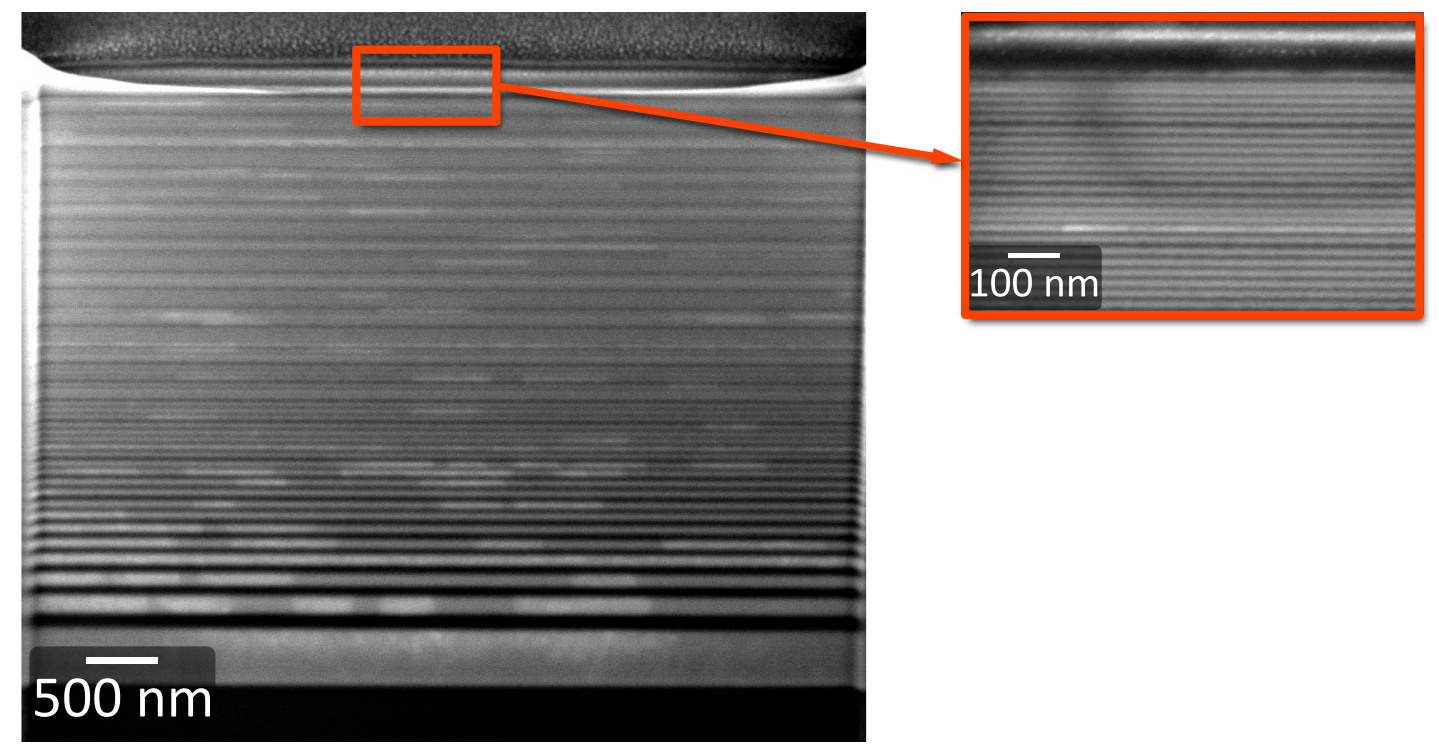

Abbildung 5.41: „bottom-up“-Deposition eines $\mathrm{Ti} / \mathrm{ZrO}_{2}-$ Multischichtpakets mit der Einhaltung der Reihenfolge nach dem Zonenplattenbildungsgesetz. Obwohl die dicksten Zonen zuerst hergestellt wurden, existiert selbst bei den dünnsten Schichten (ca. $13 \mathrm{~nm}$ ) keine kumulative Rauigkeit.

Im nächsten vorbereitenden Schritt wurde die Multischichtdeposition auf einer gekrümmten Oberfläche hinsichtlich einer Entstehung kumulativer Rauigkeiten analysiert, welche im Fall von gesputterten Schichten aufgrund einem unter flachen Einfallswinkeln auftretenden Abschattungseffekt begünstigt ist [114]. Dazu wurde die Beschichtung eines Ti-Droplets untersucht, welches zufällig während des Herstellungsprozesses auf einer bereits aufgewachsenen periodischen $\mathrm{Ti} / \mathrm{ZrO}_{2}-\mathrm{Multischicht}$ mit ca. $50 \mathrm{~nm}$ Einzelschichtdicke deponiert wurde (s. Abb. 5.42). Trotz des extrem kleinen Krümmungsradius des Droplets zeigt sich in dem REM-Querschnitt eine intakte Multischicht, die die Oberfläche des Droplets mit einer ausreichenden Haftung bedeckt. Lediglich an den Rändern ergibt sich durch einen möglichen erhöhten Wiederabtrag von Schichtmaterial unter flachen Winkeln eine niedrigere Schichtdicke. Zudem wird hier die effektiv zu beschichtende Fläche größer, sodass die gleiche Anzahl auftreffender Targetionen und -atome ebenfalls zu einer geringeren Schichtdicke führt. Die Beobachtung deponierter Droplets liefert also vielversprechende Ergebnisse, anhand derer die Beschichtung eines wesentlich größeren runden Drahtes möglich wäre. 


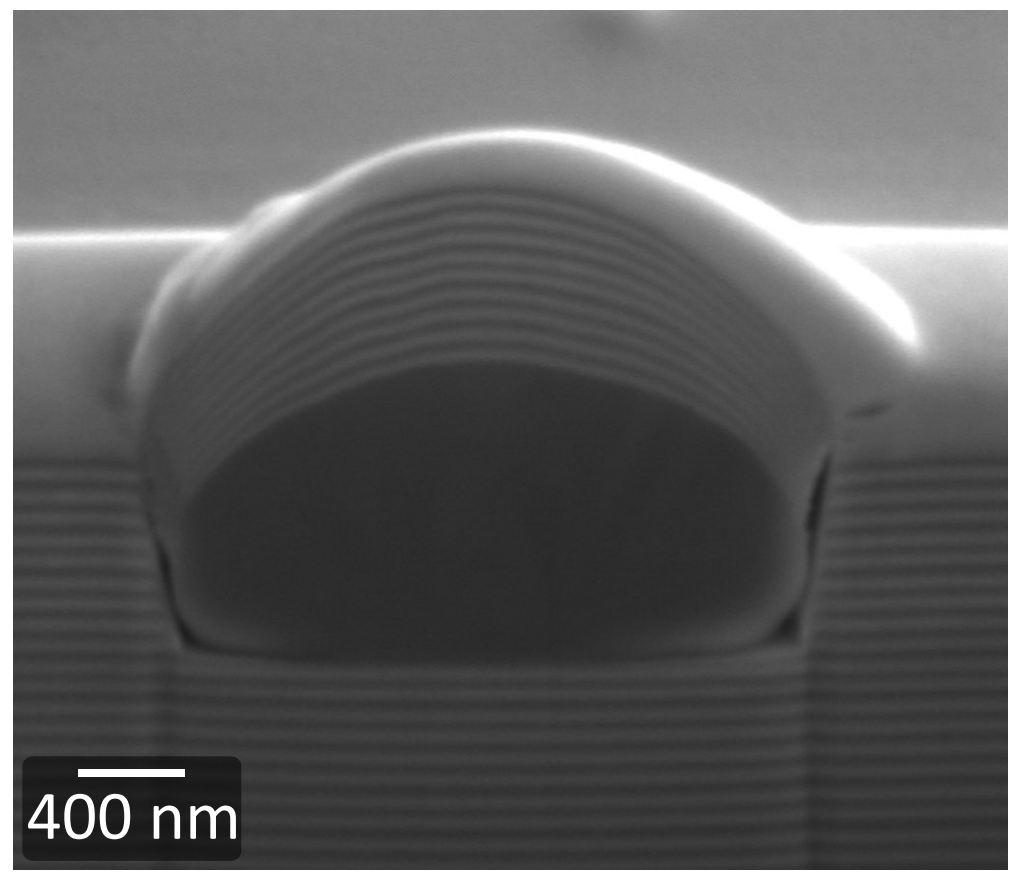

Abbildung 5.42: REM-Querschnittsaufnahme eines deponierten Ti-Droplets. Mit nach außen abnehmender Schichtdicke zeigt sich eine intakte glatte Multischichtstruktur ohne Ausbildung einer kumulativen Rauigkeit. (Die Aufnahme wurde freundlicherweise von Inga Knorr angefertigt und ist in [176] veröffentlicht.)

\subsubsection{Beschichtung rotierender Drähte}

Für die Herstellung laserdeponierter MZP wurde zunächst ein FeC-Draht verwendet, der verglichen mit den in der Literatur verwendeten Drähten $(\varnothing=18-140 \mu \mathrm{m}$, [83, 116]) einen relativ geringen Durchmesser von nur $10 \mu \mathrm{m}$ besitzt. Hierbei wurde untersucht, ob die Deposition einer stark gekrümmten Oberfläche eines rotierten Drahtes gelingt. Experimentell mussten die ersten Zonen, die eine Gesamtschichtdicke von $5 \mu \mathrm{m}$ entsprechend des Drahtradius einnehmen würden, ausgelassen werden. Mit einer Design-Brennweite von $f=350 \mu \mathrm{m}$ und der üblichen weichen Röntgenwellenlänge $(\lambda=2,879 \mathrm{~nm})$ wurden 20 Zonen aus $\mathrm{ZrO}_{2}$ und Ti mit Schichtdicken zwischen 115 und $85 \mathrm{~nm}$ hergestellt, wobei mit der Deposition von $\mathrm{ZrO}_{2}$ begonnen wurde. Dabei gilt zu berücksichtigen, dass die Depositionsrate auf einem rotierenden zylindrischen Draht $\pi$-mal kleiner ist als bei der Deposition auf einem planaren Substrat. Die benötigte Laserpulszahl wurde zunächst mit Hilfe von Schichtdickenmessungen an planaren Tiund $\mathrm{ZrO}_{2}$-Einzelschichten bestimmt und für eine geplante zirkulare Gesamtschichtdicke von ca. 1,5 $\mu \mathrm{m}$ entsprechend umgerechnet. Die Rotationsgeschwindigkeit des Drahtes 


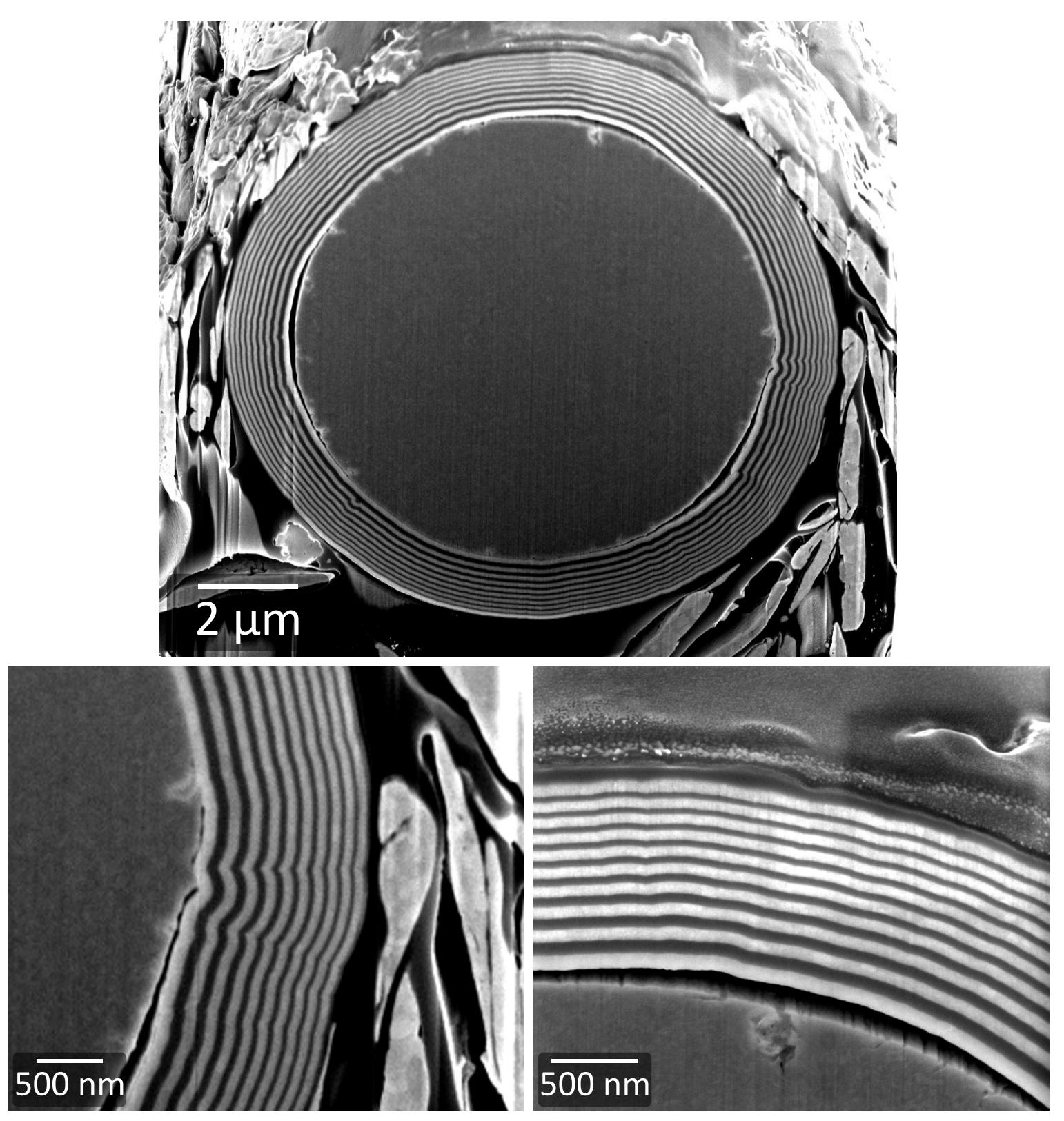

Abbildung 5.43: REM-Aufnahme einer ionenpolierten Querschnittsfläche des in Leitsilber eingebetteten FeC-Drahtes. Die Übersicht zeigt eine glatte, zirkulare Multischicht, welche ohne die Entwicklung einer kumulativen Rauigkeit aufgewachsen ist. Oberflächen- und Schichtartefakte (links) werden dank des Glättungsverhaltens von $\mathrm{ZrO}_{2}$ mit zunehmender Deposition ausgeglättet. Aufgrund unzureichender Haftung zwischen Schicht und Substrat oder zu hoher Verspannung der Multischicht ist sie teilweise vom Substrat abgelöst (rechts) [176].

wurde so gewählt, dass während einer Einzelschicht mindestens eine vollständige Umdrehung durchlaufen wird.

Abbildung 5.43 zeigt schließlich den im Rahmen dieser Arbeit ersten hergestellten Demonstrator einer MZP im Querschnitt. Auch bei einer zirkularen Deposition ist 
bei Betrachtung der Schichtstruktur kein Anzeichen einer kumulativen Rauigkeit erkennbar, sodass entgegen anderer Herstellungsmethoden wie z. B. Sputtern die Laserdeposition beliebig vieler intakter Zonen möglich ist. Darüber hinaus ist die glättende Eigenschaft des $\mathrm{ZrO}_{2}$ hervorragend geeignet, um Substrat- und Schichtartefakte sukzessive auszuglätten, wie es besonders in Abbildung 5.43 links sichtbar wird. Unglücklicherweise ist die Adhäsion zwischen Drahtoberfläche und Multischicht nicht überall ausreichend gegeben, sodass sie an drei Stellen im Abstand von $120^{\circ}$ von Substrat abgelöst ist (s. Abb. 5.43 oben u. rechts). Damit konnten die wahrscheinlich durch sog. „shot peening“-Effekte (Deposition hochenergetischer Targetionen, [180, 181]) verursachten inneren Spannungen abgebaut werden. Um die benötigten Haftungseigenschaften zu verbessern, könnte die raue und unbehandelte Drahtoberfläche z. B. durch Anätzen geglättet und gereinigt werden. Weiterhin könnten adhäsive Pufferschichten aus Cr oder Ti mit einer hohen chemischen Reaktivität als Haftvermittler deponiert werden $[182,183]$.

Da der FeC-Draht bezüglich der Rauigkeit eine nicht optimale Oberfläche besitzt, wurde zusätzlich die Beschichtung eines ca. $54 \mu \mathrm{m}$ dicken Glasdrahtes vorgenommen. Die Vorteile eines Glasdrahtes liegen in der exzellenten Zylinderform sowie einer geringen Oberflächenrauigkeit. In Abbildung 5.44 ist die Oberfläche eines Glasdrahtes dargestellt, welche zur Reinigung und Verbesserung der Haftungseigenschaften mit Chromschwefelsäure $\left(\mathrm{H}_{2} \mathrm{SO}_{4}+\mathrm{CrO}_{3}\right)$ und Aceton $\left(\mathrm{C}_{3} \mathrm{H}_{6} \mathrm{O}\right)$ chemisch vorbehandelt wurde.

Für die Draht-Beschichtung nach dem Zonenplattenbildungsgesetz wurden die Wellenlänge $\lambda=2,879 \mathrm{~nm}$ und Fokuslänge $f=1 \mathrm{~mm}$ ausgewählt. Durch Auslassen der dicksten Schichten, durch welche Zonenpositionen innerhalb des Drahtes eingenommen würden, wurden 162 Schichten des $\mathrm{Ti} / \mathrm{ZrO}_{2}$-Systems mit Dicken zwischen 27 und $25 \mathrm{~nm}$ hergestellt. Dieses Mal wurde allerdings mit der Deposition von Ti als verbesserter Haftvermittler begonnen. Während der Schichtherstellung wurde eine Energiekorrektur von $3,42 \cdot 10^{-5} \mathrm{~mJ} /$ Puls eingestellt.

In Abbildung 5.45 oben ist schließlich eine Übersicht der Drahtschnittfläche gezeigt, die nach der Multischichtdeposition im FIB erstellt wurde. Dabei lässt sich zunächst eine hohe Anzahl deponierter Droplets erkennen, sodass in diesem Fall keine dropletfreie Drahtposition für die Anfertigung eines Schnitts gefunden werden konnte. Bei der Beschichtung eines derart großen Drahtes mit einer Schichtdicke von ca. $3 \mu \mathrm{m}$ bedarf es demnach weiterer Herstellungsoptimierung zur Reduzierung der deponierten Droplets. 


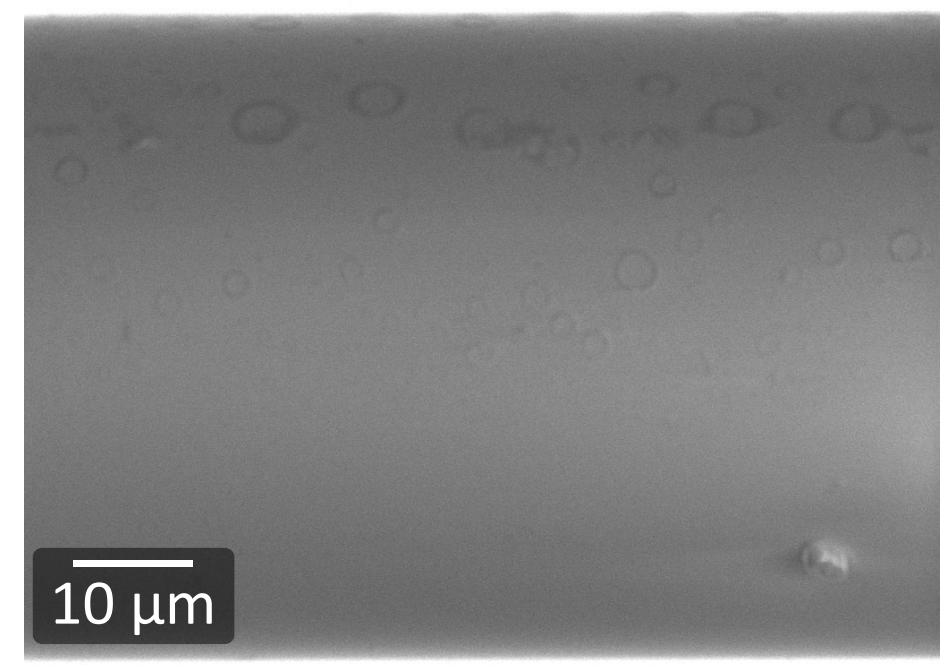

Abbildung 5.44: REM-Aufnahme einer ca. $54 \mu \mathrm{m}$ dicken Glasfaser. Durch Anätzen mit $\mathrm{H}_{2} \mathrm{SO}_{4}+\mathrm{CrO}_{3}$ und $\mathrm{C}_{3} \mathrm{H}_{6} \mathrm{O}$ wurde einer unzureichenden Haftung während der Multischichtdeposition vorgebeugt. (Die Aufnahme wurde freundlicherweise von Felix Schlenkrich angefertigt.)

Der unterschiedliche Kontrast des Glasdrahtes ist durch Aufladungserscheinungen im REM begründet.

Eine detaillierte Ansicht der $\mathrm{Ti} / \mathrm{ZrO}_{2}-$ Schichtstruktur ist in Abbildung 5.45 unten dargestellt. Hier zeigt sich die hohe und in der Literatur selten beschriebene Qualität der gekrümmten Multischichten, welche sehr glatt und ohne die Ausbildung einer kumulativen Rauigkeit aufgewachsen sind. Trotzdem lässt sich wiederum eine geringe Ablösung der Multischicht von der Drahtoberfläche erkennen, sodass auch in diesem Fall zukünftig weitere Optimierungsschritte bezüglich einer adäquaten Haftung unternommen werden müssten. Aus Zeitgründen wurde aus diesem zirkularen Schichtpaket bisher keine MZP hergestellt, weshalb zukünftig weitere Experimente zur Untersuchung der Fokuseigenschaften von MZP mit weicher Röntgenstrahlung geplant sind. Da die derzeit besten Fokussierungen harter und weicher Röntgenstrahlung im Bereich von $R=39-280 \mathrm{~nm}$ u. a. durch Verwendung von Glas als Trägermaterial erzielt wurden $[82,102,106]$, wird auch bei einer laserdeponierten MZP auf Glas eine hohe Auflösung erwartet. 

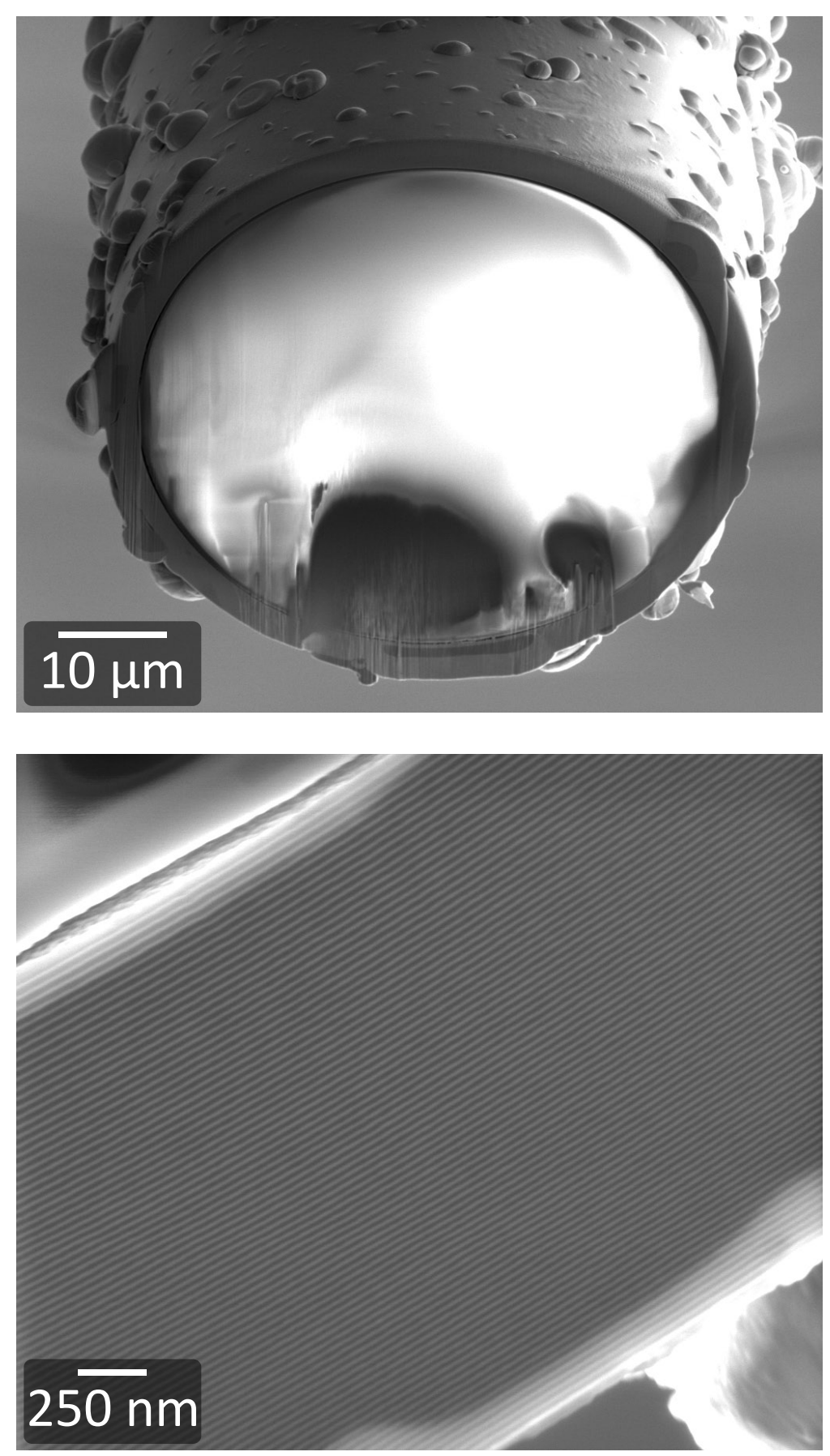

Abbildung 5.45: REM-Aufnahmen des $\mathrm{Ti} / \mathrm{ZrO}_{2}$-deponierten Glasdrahtes. Durch die hohe Anzahl an Droplets sind auch einige in dem Querschnittsbereich der sichtbaren Multischicht integriert (oben). Dennoch sind die Schichten sehr glatt ohne die Existenz einer kumulativen Rauigkeit gewachsen (unten). 


\subsection{Zusammenfassung und Ausblick}

Insgesamt hat dieses Kapitel gezeigt, wie sich auf effektive Weise multilagenbasierte Transmissionsoptiken (MLL, MLL als Monochromatoren und MZP) mittels der Kombination aus PLD und FIB erzeugen lassen. Die gepulste Laserdeposition erweist sich dabei als besonders geeignete Methode, um qualitativ hochwertige Multilagen ohne kumulative Rauigkeiten auf planaren sowie gekrümmten Substraten herzustellen. Dazu mussten grundlegende Ablations- und Depositionseigenschaften der verwendeten Multischichtsysteme untersucht, sowie gezielte experimentelle Vorbereitungen getroffen werden, um vorgegebene Multischichtdicken präzise realisieren zu können. Dank der hochenergetischen Laserdeposition spielt es dabei keine Rolle, ob die Deposition mit der dünnsten oder der dicksten Zone begonnen wird. Die Ausbildung additiver Rauigkeiten bleibt in beiden Fällen aus. Besonders bei der Beschichtung von Drähten erweist sich diese Erkenntnis zum Vorteil, um unter Vernachlässigung der Haftungsprobleme nahezu beliebig große und kleine Drähte mit beliebig vielen Zonen deponieren zu können.

In der zur Zeit laufenden Masterarbeit von Florian Döring gelang es bereits, $\mathrm{Ti} / \mathrm{ZrO}_{2}-$ Multischichten in sehr hoher Qualität auf einem nur $900 \mathrm{~nm}$ dicken WDraht herzustellen (s. Abb. 5.46). Weiterhin ist die Beschichtung von Glasdrähten mit lateralen Dickengradienten geplant, um durch globale Erfüllung der BraggBeziehung eine hocheffiziente zweidimensionale Fokussierung von Röntgenstrahlung zu ermöglichen. Bezüglich der Fabrikation von hochwertigen MZP auf großen Drähten muss allerdings die Dropletanzahl weiter minimiert werden. Eine ausreichende Haftung der Multischichten könnte durch Ätzen oder Deposition von Pufferschichten erzielt werden. Auch ein Wechsel des Materialsystems könnte in Erwägung gezogen werden.

Zur Präparation von Transmissionsoptiken erlaubt die fokussierte Ionenstrahltechnik eine flexible Mikromanipulation und sorgfältiges Polieren von Multischichtstrukturen. Weiterhin ermöglicht die Kombination von Elektronen- und Ionenstrahl die Herstellung von beam stops und die Fixierung von Strukturen mittels Pt-Deposition unter ständiger Beobachtung des in-situ REM. Mit dieser Methode sollen neben den MLL in Zukunft auch MZP fabriziert werden, indem von beschichteten Drähten einzelne Scheiben als Zonenplattenstruktur abgeschnitten und auf ein $\mathrm{Si}_{3} \mathrm{~N}_{4}$-Fenster transferiert werden. Auf diese Weise ließen sich auch zweidimensionale Röntgenmonochromatoren herstellen.

Anhand von Strahlungsexperimenten der ersten hergestellten MLL an einem „tabletop“-Röntgenmikroskop wurde eine effiziente Fokussierbarkeit weicher Röntgenstrah- 
lung mit einer Auflösung von ca. $180 \mathrm{~nm}$ demonstriert. Ein Großteil der hier aufgezeigten Optiken wurde bislang leider nicht vermessen, sodass zukünftige Experimente, auch mit weicher Synchrotronstrahlung, in Planung sind.

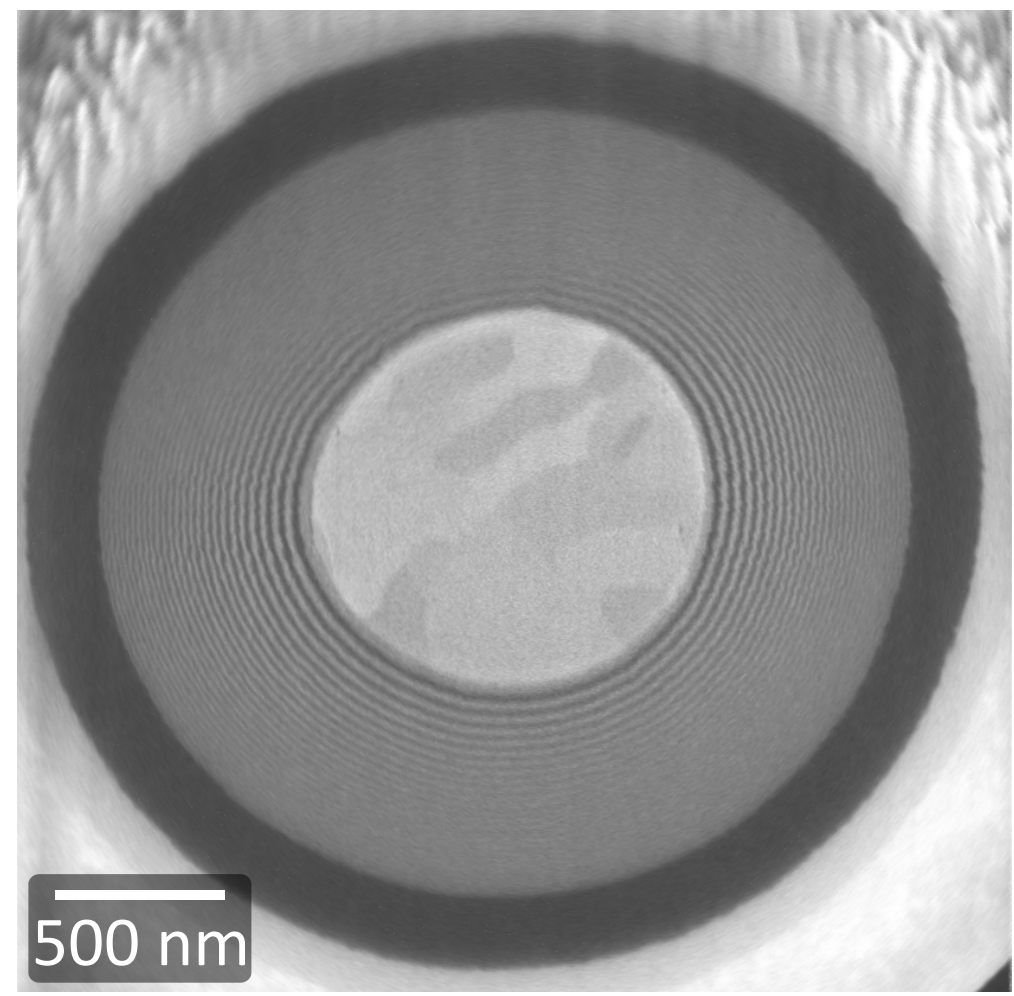

Abbildung 5.46: REM-Aufnahme eines von Florian Döring deponierten W-Drahtes mit einem Durchmesser von nur $900 \mathrm{~nm}$. Hergestellt wurden 52 Schichten $\mathrm{Ti} / \mathrm{ZrO}_{2}$ mit Dicken zwischen 31 und $11 \mathrm{~nm}$. Selbst bei einem so kleinen Krümmungsradius deutet sich keine Entstehung einer kumulativen Rauigkeit an. (Die leicht geriffelte Schichtstruktur im mittleren Bildbereich ist allein durch eine Instabilität des fixierten Drahtes während der REM-Aufnahme begründet.) 



\title{
6 Transmissionsoptiken für harte Röntgenstrahlung
}

\begin{abstract}
Im folgenden Kapitel werden die Entwicklungsstufen einer Multilagen-LaueLinse (MLL) für den harten Röntgenbereich beschrieben. Allgemein werden zunächst das Design sowie notwendige experimentelle Vorbereitungen erläutert und in späteren Abschnitten die eigentliche Multischichtherstellung mittels gepulster Laserdeposition und Fabrikation einer linienfokussierenden eindimensionalen MLL im FIB aufgezeigt. Im Anschluss folgen Messergebnisse der Strahlcharakteristik am Synchrotron, bevor im letzten Abschnitt das Design und die Herstellung einer gekreuzten Multilagen-Laue-Linse zur Erzeugung eines quasi-zweidimensionalen Punktfokus aufgezeigt wird.
\end{abstract}

\subsection{Systemauswahl und Design von Multilagen-Laue-Linsen}

Wird die Röntgenmikroskopie mit hochenergetischer, harter Röntgenstrahlung betrieben, können aufgrund ihrer hohen Eindringtiefe wesentlich dickere Proben größer als $10 \mu \mathrm{m}$ im Vergleich zur Mikroskopie mit weicher Röntgenstrahlung analysiert werden. Die damit einhergehende geringe Absorption von Strahlung in Materie erschwert allerdings die Herstellung von entsprechenden Optiken, zumal eine effiziente Fokussierung nur dann erreicht werden kann, wenn das stärker absorbierende Zonenmaterial ausreichender optischer Tiefe mit der transmittierenden Röntgenstrahlung genügend wechselwirkt. Das Ziel der in diesem Kapitel vorgestellten Untersuchungen ist die Planung und Realisierung einer MLL für harte Röntgenstrahlung, welche aus einem mittels Kirkpatrick-Baez-Spiegeloptik (KB) vorfokussierten Synchrotronstrahl durch weitere Fokussierung einen extrem kleinen Nanospot erzeugen soll (s. Abb. 6.1). Diese innovative Aneinanderreihung zweier komplementärer Optiksysteme hat es für Röntgenstrahlung in der Literatur bisher noch nicht gegeben. Dabei wird eine 
räumliche Auflösung erwartet, die unterhalb der derzeitigen Grenze von $R=7 \mathrm{~nm}$ liegt, welche mit Hilfe eines reflektiven Röntgenspiegels erreicht wurde [184]. Grundlegende konzeptionelle Schritte der hier vorgestellten Methode zur Röntgenstrahlfokussierung, die sowohl die Planung als auch die charakterisierenden Messungen am Synchrotron umfassen, wurden dabei in Zusammenarbeit mit dem Göttinger Institut für Röntgenphysik durchgeführt und sind ergänzend durch die MLL-Herstellung mittels FIB in [185] veröffentlicht. Genau wie im vorangegangenen Kapitel 5.1 wurden auch hier die Designparameter so gewählt, dass die Röntgenoptik an die Anforderungen ihres Einsatzgebiets wie Wellen- und Fokuslänge, Gesamt- und Einzelschichtdicke sowie optische Tiefe angepasst werden.

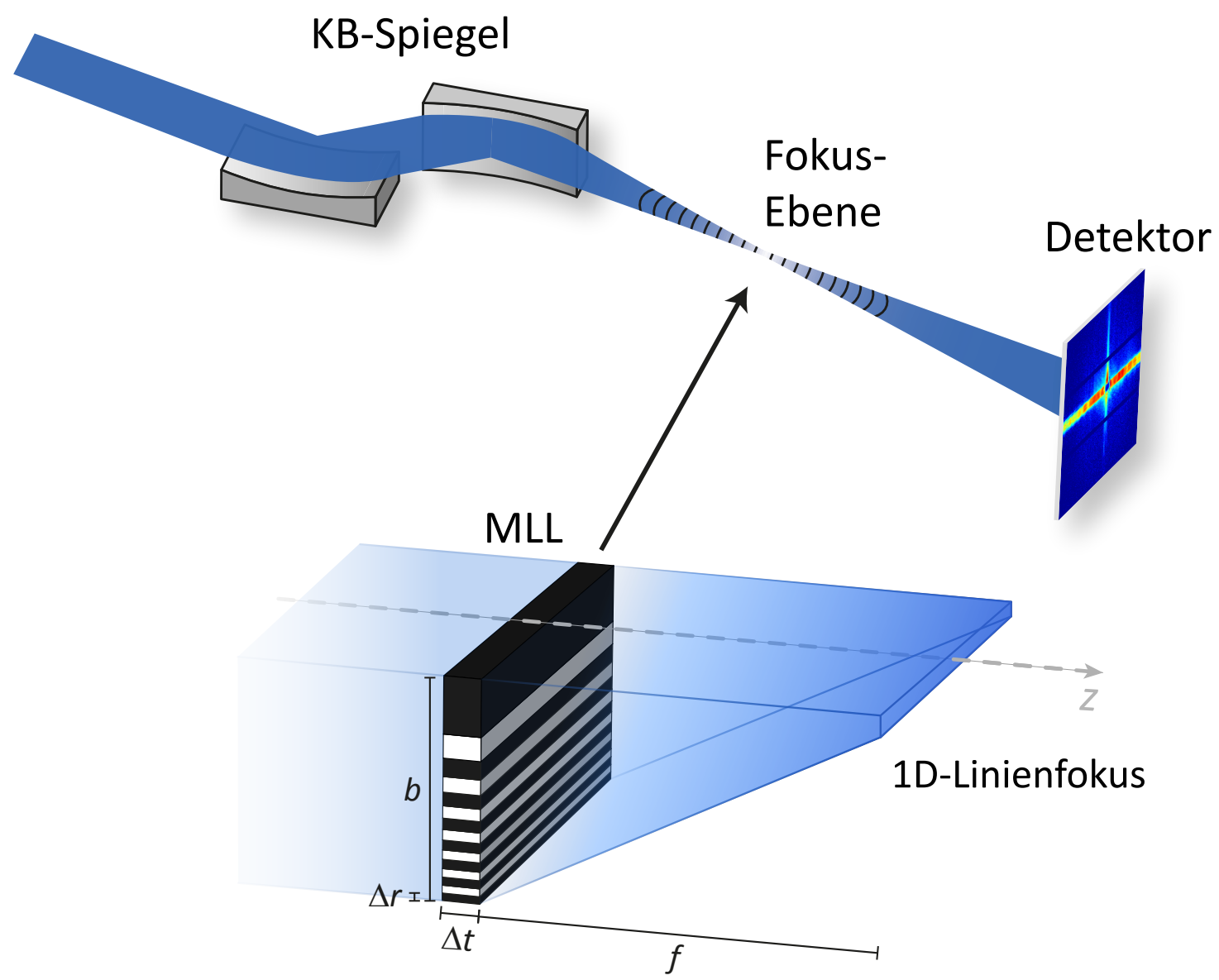

Abbildung 6.1: Experimenteller Aufbau am Strahlrohr P10 der Beamline PETRA III am DESY, Hamburg. Der durch ein kommerziell erhältliches KB-Spiegelsystem vorfokussierte Synchrotronstrahl wird auf eine im Rahmen dieser Arbeit hergestellten MLL gelenkt, um durch weitere Fokussierung einen hochaufösenden Linienfokus zu erzielen.

Die experimentellen Untersuchungen der Fokuseigenschaften wurden in diesem Fall, wie in Kapitel 3.4 beschrieben, von der AG Salditt (Inst. f. Röntgenphysik, Universität 
Göttingen) am Deutschen-Elektronen-Synchrotron DESY, Hamburg durchgeführt. Am Strahlrohr P10 der Beamline PETRA III stand dazu ein hochkohärenter Synchrotronstrahl mit einer relativ niedrig gewählten Wellenlänge $(\lambda=0,0898 \mathrm{~nm})$ zur Verfügung, um nach Gleichung 2.28 eine hohe Auflösung der beugenden Struktur zu erlangen. Bezüglich der Materialwahl werden bei multilagenbasierten Optiken für eine ausreichende Wechselwirkung von harter Röntgenstrahlung mit Materie meist schwere Elemente oder Verbindungen wie z. B. W, $\mathrm{WSi}_{2}$, Ni oder Pt als Absorbermaterial bevorzugt, während sich für den Spacer u. a. Silizide und Carbide sowie ihre reinen Elemente eignen, um einen guten optischen Kontrast zum jeweiligen Absorber herzustellen. Für das Design einer MLL für harte Röntgenstrahlung wurde die Materialkombination W/Si gewählt, welche aufgrund zeitlicher und thermischer Stabilität über $100^{\circ} \mathrm{C}$ sowie hoher Reflektivitäten häufig auch für die Herstellung von Röntgenspiegeln in diesem Wellenlängenbereich benutzt wird [40,186]. Bei $\lambda=0,0898 \mathrm{~nm}$ ergibt sich zwar eine insgesamt geringe Absorption von W und Si (s. Abb. 6.2), die sich aber dennoch aufgrund der Tatsache, dass sich die gewählte Wellenlänge neben einer Absorptionskante von W befindet, um ungefähr zwei Größenordnungen unterscheidet und somit zu einem ausreichenden optischen Kontrast der beiden Materialien führt.

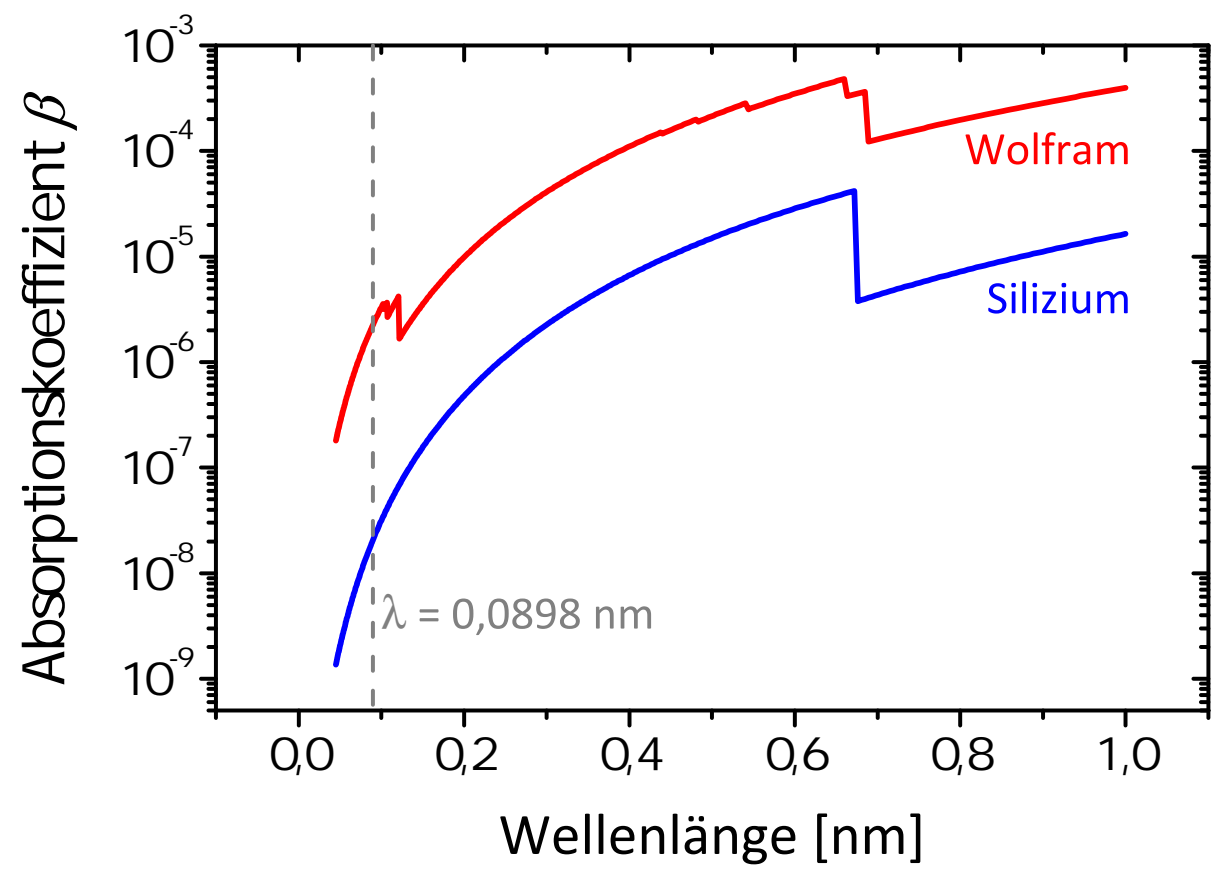

Abbildung 6.2: Absorptionskoeffizienten von W und Si in Abhängigkeit der Wellenlänge harter Röntgenstrahlung. Links neben der Absorptionskante von W ergibt sich bei der im Experiment verwendeten Wellenlänge von $\lambda=0,0898 \mathrm{~nm}$ zu dem durchlässigeren Si ein Unterschied über zwei Größenordnungen. 
Neben der festen Wellenlänge bestimmte weiterhin die Größe des Synchrotronstrahls das Design der MLL, an welche die Gesamtschichtdicke der Linse für eine optimale Ausleuchtung angepasst werden musste. Aufgrund der maximalen Quellgröße im KBFokus $(300-400 \mathrm{~nm})$ wurde die Größe der Linse auf $b=400 \mathrm{~nm}$ Gesamtschichtdicke begrenzt. Um mit einer derart kleinen MLL dennoch eine große numerische Apertur und somit eine hohe Auflösung in der 1. Beugungsordnung zu erreichen, wurde eine geringe Brennweite gewählt (vgl. Abb. 2.5). Da sämtliche Charakterisierungen ausschließlich im Fernfeld und nicht durch knife-edge-Messungen (s. Kap. 5.1) stattfinden sollten, konnte in diesem Fall eine extrem kurze Brennweite von $f=14,7 \mu \mathrm{m}$ gewählt werden. Da hier nicht von einer vollständigen Beleuchtung der MLL ausgegangen wurde (je nach Quellgröße wurden die dicksten Schichten ausgelassen), stellt diese Brennweite gleicherweise die kürzeste Entfernung von der Linse dar, ab dem der resultierende Fokus vom Nullstrahl der KB-Optik klar getrennt werden kann.

Fokus- und Wellenlänge bestimmen nach dem binären Zonenplattenbildungsgesetz (Gl. 2.8) die einzelnen Schichtdicken, welche in Tabelle 6.1 für die dicksten und dünnsten Zonen aufgelistet sind. Bis zu einer Gesamtschichtdicke von $400 \mathrm{~nm}$ lassen sich maximal 122 Zonen mit einer äußeren Zonenbreite von $\Delta r=1,65 \mathrm{~nm}$ herstellen. Wird bei der Abschätzung der bestmöglichen Auflösung von einer vollen Ausleuchtung aller Zonen ausgegangen, so wäre in diesem Fall eine theoretisch berechnete Auflösung von $R_{\text {ber. }}=2 \cdot 1,22 \Delta r \simeq 4 \mathrm{~nm}$ denkbar. Im oberen Teil der Abbildung 6.3 ist diesbezüglich eine von Aike Ruhlandt (Inst. f. Röntgenphysik, Universität Göttingen) durchgeführte Simulation der Intensitätsverteilung der Strahlpropagation hinter einer idealen MLL gezeigt [185, 187], bei der neben dem Fokus 1. Ordnung auch höhere Beugungsordnungen schwächerer Intensität sowie ein divergierender Anteil negativer Ordnungen $\mathrm{zu}$ beobachten sind. Innerhalb der Fokusebene ergibt sich anhand eines senkrecht zur Ausbreitungsrichtung der gebeugten Röntgenstrahlung gelegten Linienscans eine theoretische Auflösung von $R_{\text {sim. }}=3,8 \mathrm{~nm}$ (s. Abb. 6.3 unten), welche in guter Übereinstimmung mit der obigen Abschätzung der Aufösung steht. Informationen über reelle Grenzflächenrauigkeiten sind hierbei nicht in die Simulation miteinbezogen worden.

Um einen aufösungsmindernden Schichtdickenfehler bzw. eine Fehlpositionierung der dünneren Schichten möglichst gering zu halten, wurde bei der Herstellung der MLL mit der dünnsten Schicht begonnen. Während die Wahl des Anfangsmaterials bezüglich des Schichtwachstums keine Rolle spielt, ist es sinnvoll, dass die letzte und dickste Schicht aus Si besteht. Durch eine geringere Absorption verglichen mit 


\begin{tabular}{cccl}
\hline $\mathrm{n}$ & $d[\mathrm{~nm}]$ & $\mathrm{n}$ & $d[\mathrm{~nm}]$ \\
\hline & & & $\vdots$ \\
1 & 36,34 & \multicolumn{3}{l}{$\vdots$} \\
2 & 15,05 & 103 & 1,80 \\
3 & 11,55 & 104 & 1,79 \\
4 & 9,74 & 105 & 1,78 \\
5 & 8,58 & 106 & 1,77 \\
6 & 7,76 & 107 & 1,76 \\
7 & 7,13 & 108 & 1,75 \\
8 & 6,64 & 109 & 1,74 \\
9 & 6,24 & 110 & 1,74 \\
10 & 5,90 & 111 & 1,73 \\
11 & 5,61 & 112 & 1,72 \\
12 & 5,36 & 113 & 1,71 \\
13 & 5,14 & 114 & 1,71 \\
14 & 4,95 & 115 & 1,70 \\
15 & 4,77 & 116 & 1,69 \\
16 & 4,62 & 117 & 1,68 \\
17 & 4,47 & 118 & 1,68 \\
18 & 4,34 & 119 & 1,67 \\
19 & 4,23 & 120 & 1,66 \\
20 & 4,12 & 121 & 1,66 \\
21 & 4,01 & 122 & 1,65 \\
\hline
\end{tabular}

Tabelle 6.1: Nach dem binären Zonenplattenbildungsgesetz berechnete Einzelschichtdicken der geplanten MLL für $\lambda=0,0898 \mathrm{~nm}$ und $f=14,7 \mu \mathrm{m}$. Aufgrund des wurzelförmigen Abfalls zeigen sich die dünneren Schichtdicken (rechte Spalte) annähernd periodisch.

W ist dadurch eine lokale Effizienzerhöhung zu erwarten. Letztere soll weiterhin durch eine Verkippung der MLL um einen mittleren Winkel von 22 mrad relativ zur optischen Achse maximiert werden. Somit ist die Einhaltung der Bragg-Bedingung für äußere Zonen gegeben, welche für eine Schichtperiode von ca. $2 \mathrm{~nm}$ optimiert ist. Die Gesamteffizienz hingegen lässt sich maßgeblich durch die richtige Wahl der optischen Tiefe beeinflussen. Da im FIB nahezu beliebige Aspektverhältnisse realisierbar sind, wurde für die beugende Multischichtstruktur eine nach Gleichung 2.24 berechnete optische Tiefe von $\Delta t=3,256 \mu \mathrm{m}$ angenommen, um einen Phasenschub von $\pi \mathrm{zu}$ erzeugen. Darüber hinaus wurde geplant, einen weiteren Teil dieses aperiodischen Schichtpakets wesentlich dünner $(\Delta t<200 \mathrm{~nm})$ und somit als abzubildende Probe anzufertigen, um Fokussierungseigenschaften anderer Optiken untersuchen zu können. 

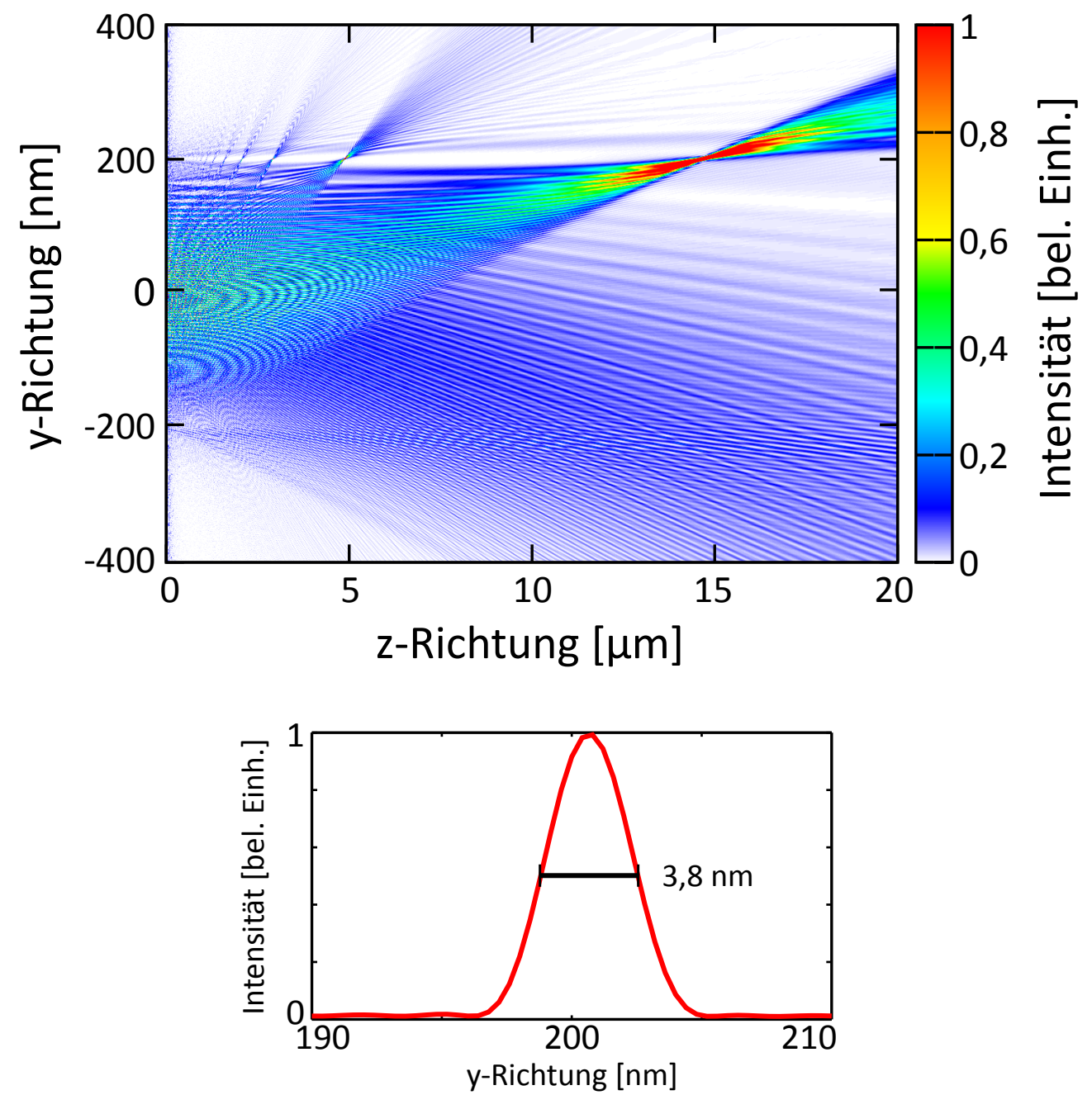

Abbildung 6.3: Simulierter Strahlengang hinter einer dünnen MLL mit $\lambda=0,0898 \mathrm{~nm}, f=14,7 \mu \mathrm{m}$ und $b=400 \mathrm{~nm}$. Neben dem strahlungsintensiven Fokus 1. Beugungsordnung sind zusätzlich höhere Beugungsordnungen niedrigerer Intensität und ein divergierender Anteil des einfallenden Röntgenstrahls erkennbar (oben). Der Linienscan durch den Fokus (unten) liefert eine theoretische Auflösung von 3,8 nm. (Die Simulationsdaten wurden freundlicherweise von Aike Ruhlandt zur Verfügung gestellt [185,187].)

\subsection{Vorbereitende Untersuchungen am W/Si-System}

Um dieses komplexe Materialsystem mit exakt definierten Schichtdicken mittels gepulster Laserdeposition umzusetzen, musste zunächst eine Optimierung der Herstellungsbedingungen vorgenommen werden. W/Si ist ein sehr komplexes und nicht einfach herzustellendes System mit verschiedenen physikalischen Eigenschaften der Einzelkomponenten bezüglich der Laserablation und des Schichtwachstums, welche in den folgenden Teilabschnitten separat betrachtet werden sollen. 


\subsubsection{W- und Si-Einzelschichten}

Zunächst wird die Beziehung der unterschiedlichen physikalischen Eigenschaften anhand des binären Phasendiagramms von W und Si erläutert [188]. In diesem System mit einem großen Unterschied der Schmelztemperaturen (ca. $2000{ }^{\circ} \mathrm{C}$ ) und einer stark begrenzten Randlöslichkeit zeigen sich mehrere Eutektika bei 0,8 at\%, 40,7 at\% und 68,5 at\% W, bei denen die Erstarrung aus der Schmelze ohne Koexistenz von flüssiger und fester Phase vonstatten geht (s. Abb. 6.4). Zudem deutet die Existenz zweier intermetallischer Phasen $\left(\mathrm{Si}_{2} \mathrm{~W}\right.$ als Strichphase und $\left.\mathrm{Si}_{3} \mathrm{~W}_{5}\right)$ auf eine attraktive Wechselwirkung der beiden Materialien hin. Thermodynamisch begünstigt lässt sich daher bei den meisten hochschmelzenden Metall/Si-Systemen die Bildung einer diffusionsgetriebenen Zwischenschicht beobachten [189].

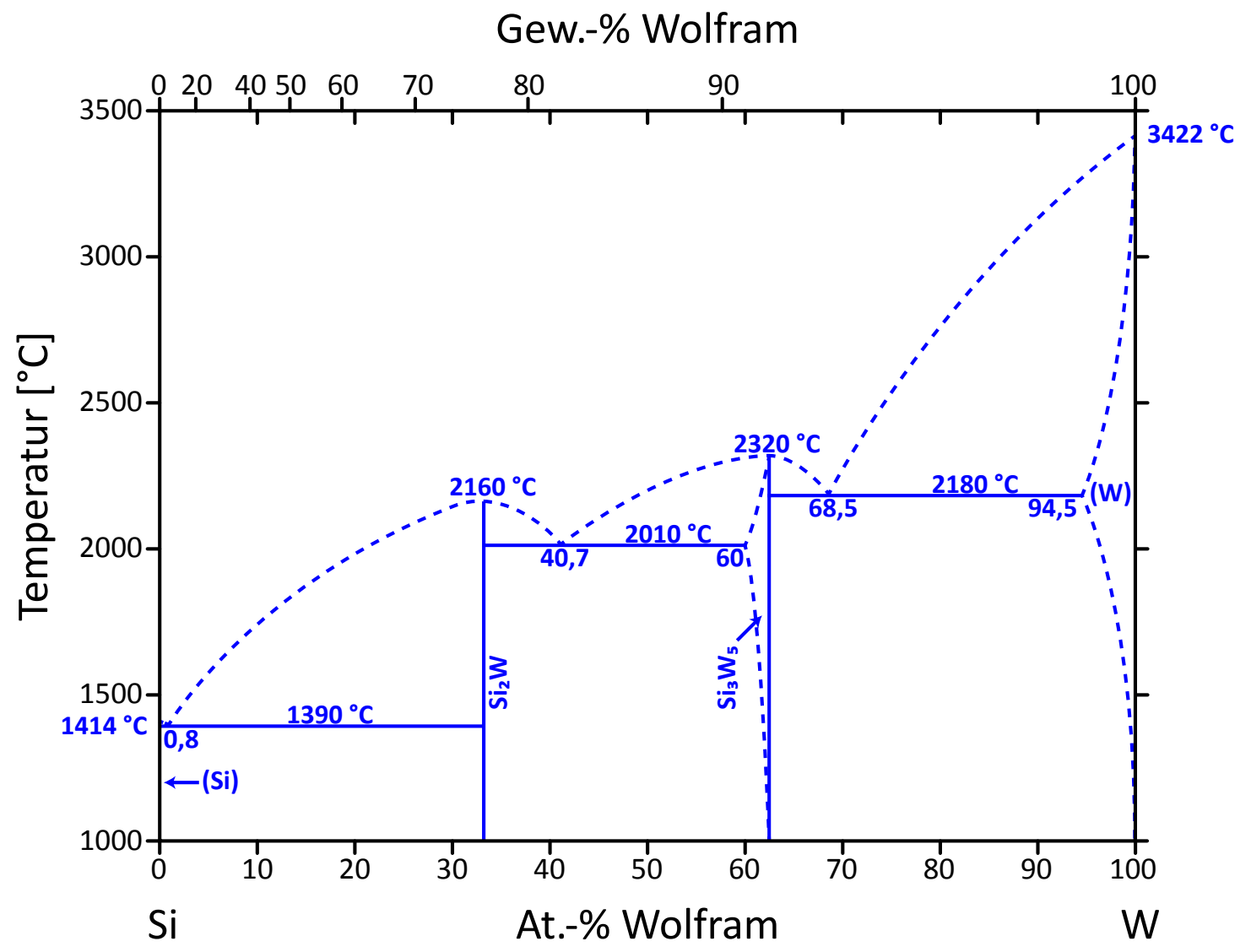

Abbildung 6.4: Binäres Phasendiagramm von W und Si, welches sich durch einen großen Unterschied der Schmelzpunkte der reinen Materialien und durch das Auftreten intermetallischer Phasen kennzeichnet (nach [188]).

Aufgrund der stark unterschiedlichen Schmelzpunkte von $\mathrm{W}\left(3422^{\circ} \mathrm{C}\right)$ und $\mathrm{Si}$ $\left(1410^{\circ} \mathrm{C}\right)$ war $\mathrm{zu}$ vermuten, dass für die laserdeponierte Multischichtherstellung 
verschieden hohe Energiedichten erforderlich sind, um einen optimalen Materialabtrag zu gewährleisten. Gleichzeitig ist wichtig, dass bei der Deposition eine zu starke TargetAufrauung vermieden wird, um die Laserleistung sowie die Langzeitstabilität der Raten nicht verfrüht herabzusetzen. Speziell beim hochschmelzenden $\mathrm{W}$ konnte anfangs mit den gleichen Herstellungsparametern, die für $\mathrm{Ti}, \mathrm{ZrO}_{2}$ und $\mathrm{MgO}$ verwendet wurden, kein Abtrag von Targetmaterial erreicht werden. W besitzt bei $248 \mathrm{~nm}$ Laserwellenlänge eine relativ niedrige optische Eindringtiefe von $\alpha^{-1}=7 \mathrm{~nm}$ sowie einen hohen Reflexionskoeffizient von $R=0,51$ [190], sodass nur die Hälfte der eingestrahlten Laserenergie tatsächlich im Target deponiert wird und einen Materialabtrag bewirken kann. Erst durch im Folgenden gezeigte Optimierungen konnte die Energieeinkopplung soweit konzentriert werden, dass ein Abtrag möglich wurde.

Dazu wurden bei zwei festen Laserenergien, die aufgrund der unterschiedlichen Schmelztemperaturen für $\mathrm{W}$ und $\mathrm{Si}$ möglichst hoch bzw. tief gewählt wurden $\left(E_{\mathrm{W}}=125 \mathrm{~mJ}, \quad E_{\mathrm{Si}}=75 \mathrm{~mJ}\right)$, mittels Verschiebung der Fokussierungslinse des Laserstrahls die Energiedichten variiert und die entsprechenden Schichtdicken nach jeweils 1000 Pulsen pro Linsenposition mittels Depositionsratenmonitor ausgelesen (s. Abb. 6.5). Die Längenachse kennzeichnet in diesem Zusammenhang den Abstand des Linsenfokus vom Target. Zunächst wird deutlich, dass sich Si bereits mit einem wesentlich weniger fokussierten Laserstrahl abtragen lässt, wohingegen eine Ablation von $\mathrm{W}$ bereits oberhalb eines Abstandes von knapp $5 \mathrm{~cm}$ infolge einer zu geringen Energiedichte nicht mehr registriert werden kann (Ablationsschwelle). Mit zunehmender Fokussierung der UV-Strahlung (von rechts nach links betrachtet) steigen die Depositionsraten beider Materialien mit einer Verkleinerung der Brennfleckgröße linear an, bis bei Si ein Maximum erreicht wird. Ab diesem Bereich kann die Rate aufgrund des sog. „shielding“-Effekts [163] nicht weiter erhöht werden, bei dem die gesättigte gasförmige Knudsenlage oberhalb der Targetoberfläche einen Großteil des Laserpulses absorbiert und damit das Abdampfen weiterer Targetatome verhindert. Durch stärkere Fokussierung nimmt sodann die gemessene Depositionsrate aufgrund der verkleinerten Brennfleckgröße wieder ab. Bei W hingegen reicht die eingekoppelte Energie pro Fläche nicht aus, um in den Sättigungsbereich der Depositionsrate zu gelangen. Da die Fokussierungslinse während der $\mathrm{W} / \mathrm{Si}$-Schichtherstellung nicht verfahren werden konnte, musste eine für beide Materialien adäquate Linsenposition gefunden werden. Sie wurde auf einen Fokusabstand von 2,6 cm festgesetzt, bei dem sich die Depositionsrate von $\mathrm{W}$ auf $0,0054 \mathrm{~nm} / \mathrm{Puls}$ und die von Si auf 0,022 nm/Puls (bei oben angegebenen Laserenergien) beläuft. 


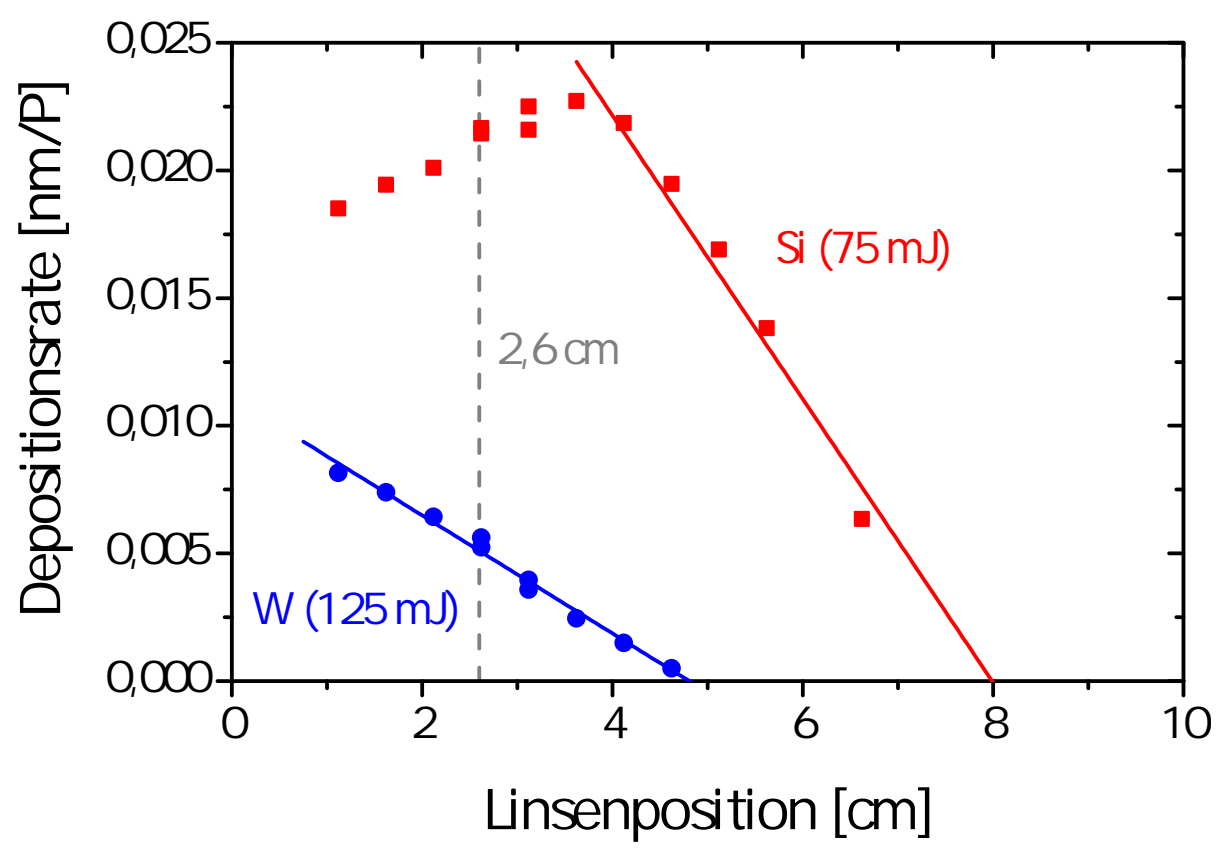

Abbildung 6.5: Depositionsraten von W und Si in Abhängigkeit der Position der Fokussierungslinse des eindringenden Laserstrahls, wobei die Längenangabe dem Abstand der Brennweite zum Target entspricht. Das Maximum der Rate, welches innerhalb der erfassten Messdaten nur für Si erreicht wird, ist durch die gesättigte Gasphase der Knudsenlage bestimmt. Für zukünftige Schichtherstellungen wurde eine feste Linsenposition von $2,6 \mathrm{~cm}$ gewählt.

Um die Depositionsraten in Abhängigkeit eines für die Laserdeposition typischen Wertes der Energiedichte $\rho=E / A$ darzustellen, wurden die Brennfleckgrößen $A$ bei verschiedenen Linsenstellungen des fokussierten Laserstrahls auf einem unbenutzten Si-Target ermittelt. Abbildung 6.6 zeigt den linearen Zusammenhang zwischen UVbestrahlter Fläche und Linsenposition auf, wobei die Nullposition den kleinstmöglichen Brennfleck mit ca. 3,5 mm² Größe im Abstand der Linsenbrennweite zeigt. Der teilweise große Fehler äußert sich durch eine erschwerte Auswertung anlässlich der weniger stark bestrahlten Randbereiche der rechteckigen Brennfleckprofile.

Abbildung 6.7 verdeutlicht schließlich den Unterschied der auf die Brennfleckgrößen normierten Depositionsraten für W und Si in Abhängigkeit der Energiedichte. Durch eine lineare Anpassung lassen sich die Ablationsschwellen, d.h. die minimal benötigten Energiedichten für einen beginnenden Abtrag beider Materialien bestimmen. Während von $\mathrm{Si}$ bereits ab $1,2 \mathrm{~J} / \mathrm{cm}^{2}$ Targetmaterial abgetragen werden kann, ist für das hochschmelzende $\mathrm{W}$ mit 2,44 J/ $\mathrm{cm}^{2}$ mehr als das Doppelte nötig, um einen dünnen Film herstellen zu können. Im Fall von Si erreicht die Depositionsrate ihr Maximum bei ca. $1,65 \mathrm{~J} / \mathrm{cm}^{2}$ und steigt durch weitere Erhöhung der Energiedichte nicht mehr 


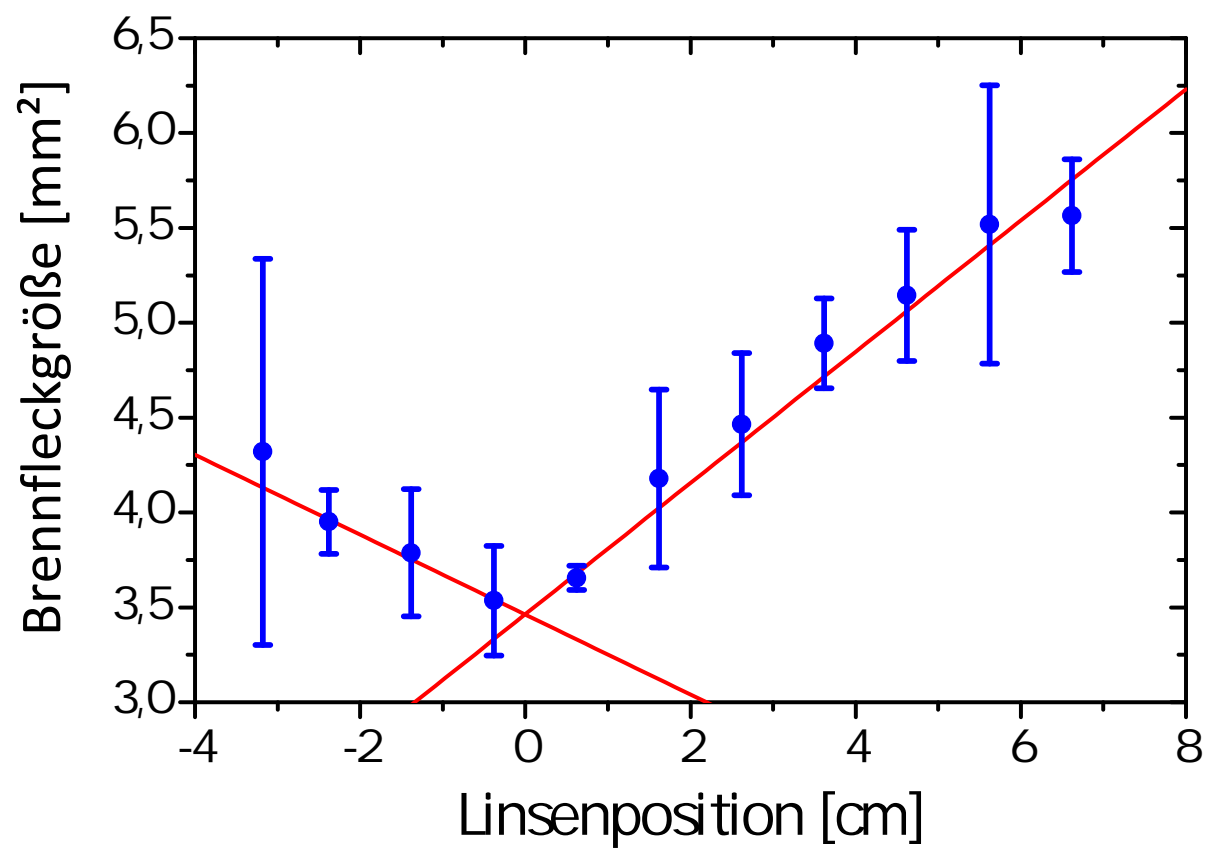

Abbildung 6.6: Verschieden stark fokussierte Strahlprofile des Lasers in Abhängigkeit der Linsenposition. In beiden defokussierten Richtungen zeigt sich ein linearer Zusammenhang zwischen bestrahlter Fläche und Linsenstellung.

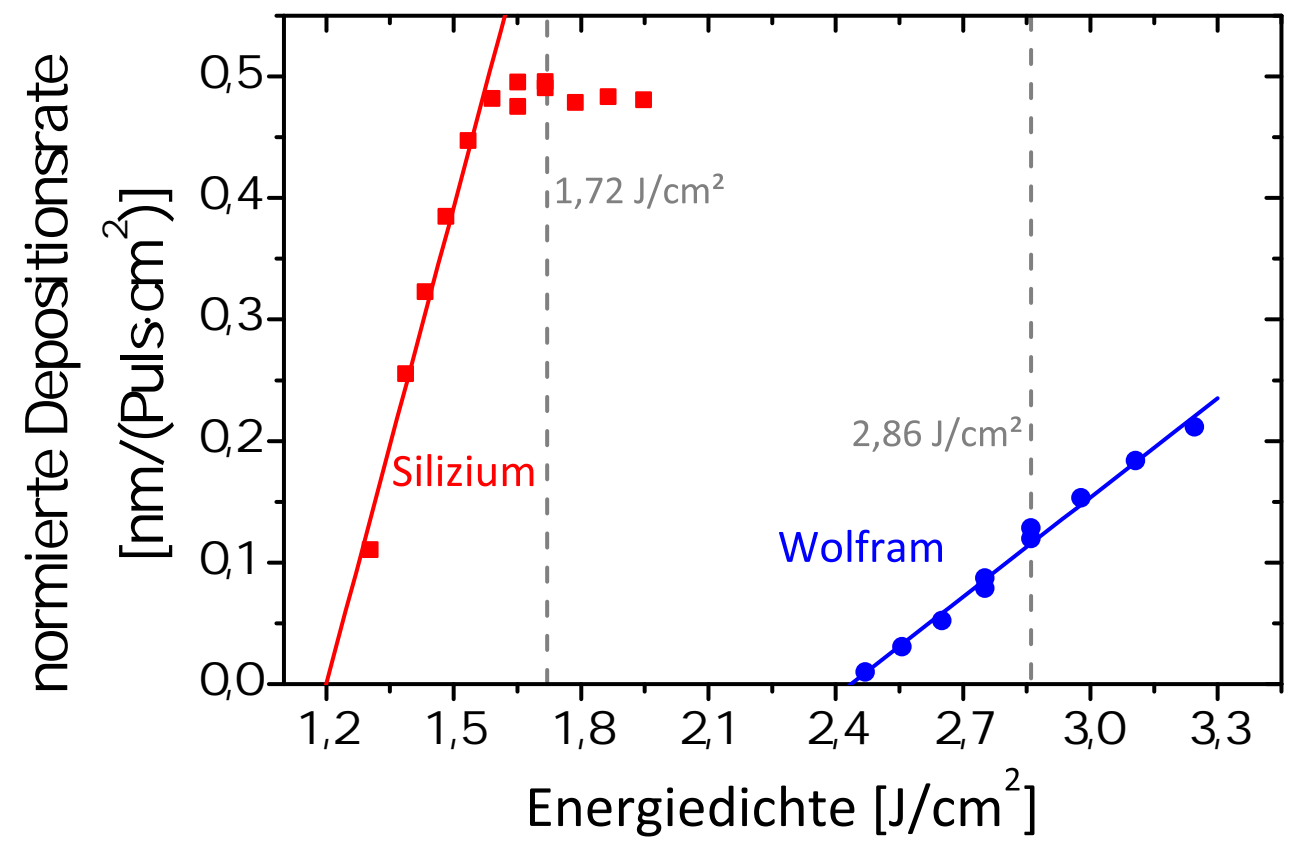

Abbildung 6.7: Normierte Depositionsraten von W und Si bei festen Laserenergien, dargestellt in Abhängigkeit der Energiedichte. Im Vergleich zu W lässt sich Si bereits mit der Hälfte der benötigten Energiedichte bishin zu einer maximalen Depositionsrate von $0,5 \mathrm{~nm} /\left(\mathrm{Puls} \cdot \mathrm{cm}^{2}\right)$ abtragen. 
an (,shielding““-Effekt, s. o.). Die gewählten Energiedichteparameter belaufen bei einer festen defokussierenden Linsenposition von 2,6 cm und Laserenergien von $E_{\mathrm{W}}=125 \mathrm{~mJ}$ und $E_{\mathrm{Si}}=75 \mathrm{~mJ}$ schließlich auf $\rho_{\mathrm{W}}=2,86 \mathrm{~J} / \mathrm{cm}^{2}$ bzw. $\rho_{\mathrm{Si}}=1,72 \mathrm{~J} / \mathrm{cm}^{2}$. Da sich Si bei dieser Energiedichte bereits im gesättigten Bereich befindet, führt die zusätzliche Absorption von Laserstrahlung zu einem großen Ionenanteil des Laserplasmas bzw. einem hohen Ionisationsgrad und somit zu einer hohen kinetischen Energie der SiIonen (bis $150 \mathrm{eV}$ ) [132]. Dabei ist bekannt, dass hochenergetische Ionen in eine bereits aufgewachsene Schicht implantiert werden und zudem einen Absputtereffekt hervorrufen können. W hingegen befindet sich bei dem gewählten Parameter nahe an der Ablationsschwelle, sodass trotz der im Vergleich zu Si höheren Energiedichte der Anteil an W-Ionen klein sein muss. Daher lässt sich vermuten, dass ein großer Teil an W-Atomen mit kinetischen Energien zwischen 5 und $10 \mathrm{eV}$ lediglich auf der Schichtoberfläche deponiert und nicht implantiert wird.

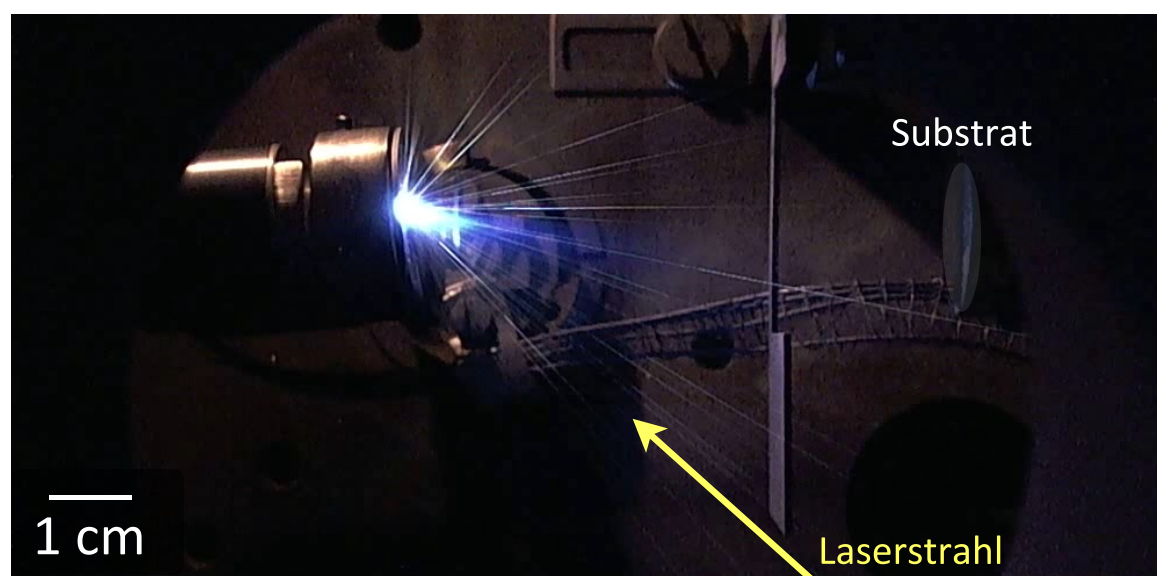

Abbildung 6.8: Gepulste Laserdeposition von W: Neben der Plasmakeule ist ein deutlicher Funkenflug heiß glühender Targetsegmente erkennbar, der teilweise bis zum Substrat hin reicht.

Allerdings ließ sich bei Beobachtung des Ablationsprozesses bei jeder im möglichen Bereich gewählten Energiedichte bereits nach einmaligem Abrastern einer frischen WTargetoberfläche durch den fokussierten Laserstrahl ein starker Funkenflug erkennen, der auf eine Ablation fester, heiß glühender Targetsegmente schließen lässt (s. Abb. 6.8). Um die Ursache dieses Funkenflugs klären zu können, wurde die Targetoberfläche eines laserbestrahlten W-Targets auf mikroskopischer Skala untersucht (s. Abb. 6.9 links). Zunächst fällt auf, dass an einigen Stellen des polykristallinen W das Targetmaterial teilweise abgelöst und verkrümmt worden ist. Dieses Phänomen wird in der Literatur als „exfoliational sputtering“ beschrieben [166], welches bei der gepulsten Laserdeposition von wenig dichten, hochschmelzenden Materialien wie $\mathrm{W}$ einen der dominierenden 

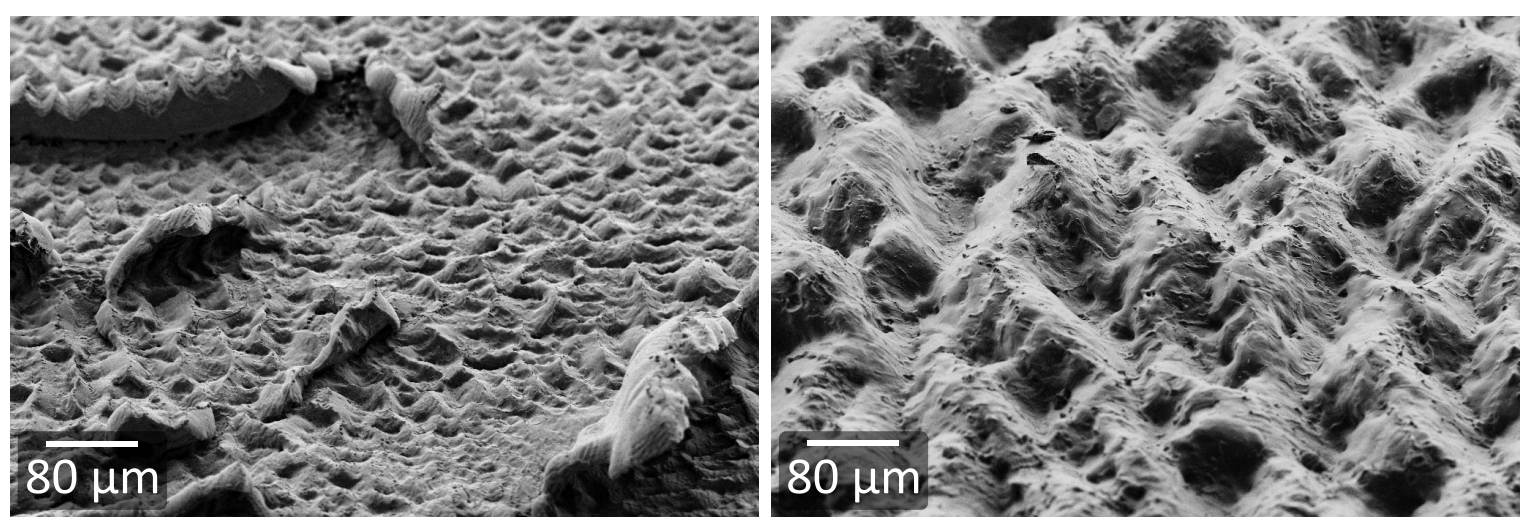

Abbildung 6.9: Verkippte REM-Aufnahmen der durch Laserbestrahlung veränderten Targetoberflächen von W (links) und Si (rechts). Während sich beim W-Target grobe Strukturen ablösen und den in Abb. 6.8 dargestellten Funkenflug verursachen, zeigt sich bei einer gleichmäßigen Wellenstruktur des Si vereinzelte Tröpfchenbildung.

Prozesse darstellt. Ein solches meist unvollständiges Abblättern wird durch lokale, sich wiederholende thermische Schocks verursacht, bei denen die laserinduzierte Temperatur, mitunter durch einen hohen thermischen Ausdehnungskoeffizienten begründet, die Schmelztemperatur des abzutragenden Materials nicht übersteigt. Durch weitere intensive UV-Bestrahlung wird die Targetoberfläche teilweise aufgebrochen und schließlich eine Ablation größerer Targetfragmente im festen Zustand herbeigeführt, worin der Ursprung des Funkenflugs gesehen wird. Bei den Untersuchungen von KeLLY et al. reicht am Beispiel von $\mathrm{W}$ eine Energiedichte von $2,5 \mathrm{~J} / \mathrm{cm}^{2}$ nicht aus, um einen vollständigen Schmelzvorgang des Targetmaterials zu bewirken [164]. Ein direktes Aufschmelzen tritt im Wesentlichen nur durch eine lokale Temperaturerhöhung an den Spitzen der abgeblätterten Strukturen auf. Hier kann bei höheren Energiedichten ab $2,5 \mathrm{~J} / \mathrm{cm}^{2}$, wie es bei denen im Rahmen dieser Arbeit gewählten Herstellungsparametern der Fall ist, sog. „hydrodynamisches Sputtern“ zur Entstehung von Droplets führen. Zwischen diesem gröberen Gefüge ist die Oberfläche gleichmäßig in einer Wellenstruktur aufgeraut und durch einzelne Spannungsrisse versetzt. Eine ähnliche Wellenstruktur, allerdings auf einer größeren Skala, zeigt ebenso die Targetoberfläche des einkristallinen $\mathrm{Si}(111)$-Targets (s. Abb. 6.9 rechts). Zu erkennen sind außerdem die einzelnen wiedererstarrten Wellenfronten, an deren Spitzen sich eine leichte Tröpfchenbildung abzeichnet.

Obwohl der oben beschriebene am W-Target entstehende Funkenflug im Bereich der möglichen Energiedichten nicht vollständig unterdrückt werden konnte, zeigt sich bei Betrachtung einer Einzelschichtoberfläche im REM (s. Abb. 6.10) neben einigen erstarrten Droplets lediglich die Existenz weniger W-Körner, die im festen Zustand 

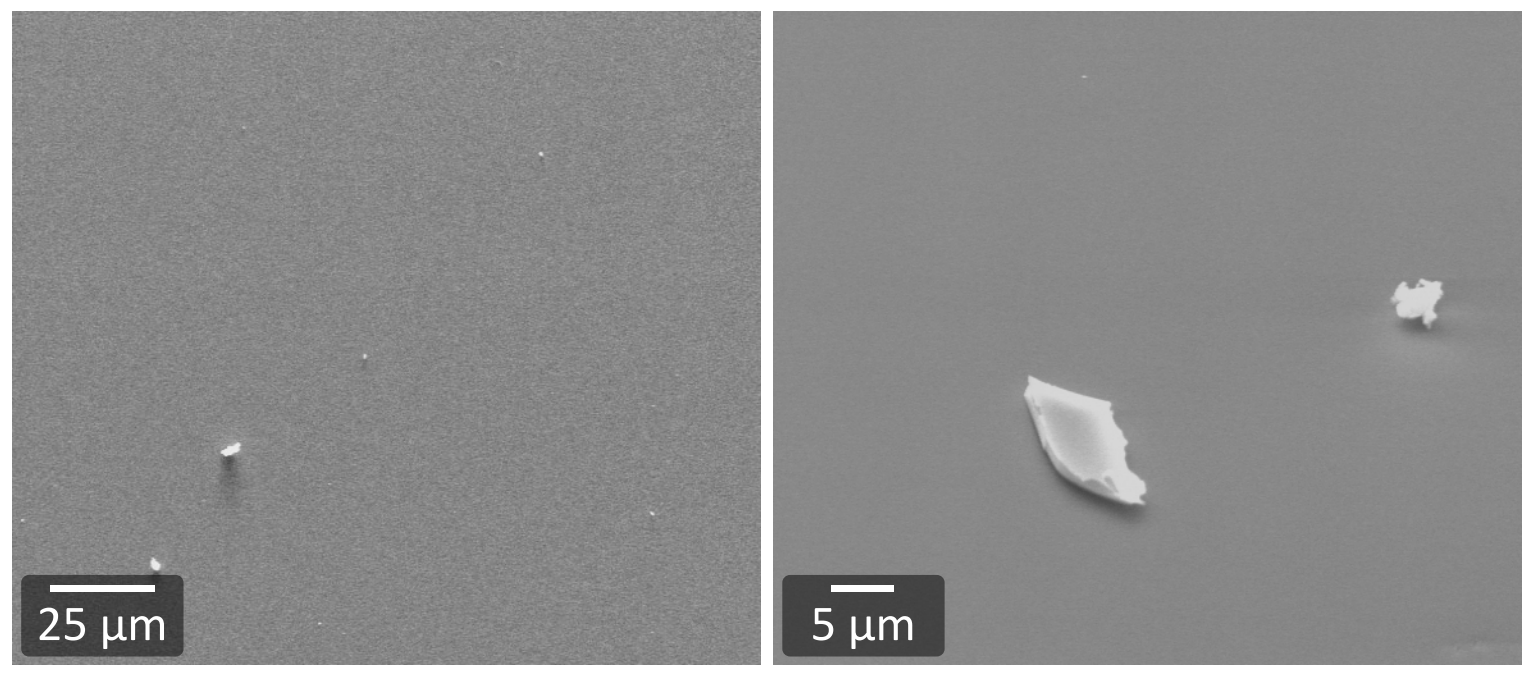

Abbildung 6.10: REM-Abbildung einer ca. 12 nm dicken W-Schicht unterschiedlicher Vergrößerungen. Neben wenigen Droplets (links) lassen sich nicht geschmolzene Teilchen erkennen (rechts), die im festen Zustand aus dem Target gelöst und durch thermisch verursachte Schockwellen bis zum Substrat hin transportiert wurden. (Die Schichtherstellung und die REM-Analyse wurden freundlicherweise in der begleitenden Masterarbeit von Christian Eberl durchgeführt.)

auf die Substratoberfläche transferiert wurden. Dies lässt sich dadurch erklären, dass einzelne Körner des kommerziell bezogenen W-Targets während der Herstellung durch festes Verpressen von Pulver bei Temperaturen unterhalb der Schmelztemperatur nicht gut miteinander verbunden worden sind, sodass durch intensive Laserbestrahlung der Targetoberfläche ganze Körner herausgerissen werden können. Die Anzahl herkömmlicher W-Tröpfchen beträgt lediglich 1,44 pro $1000 \mu \mathrm{m}^{2}$ Oberfläche bei einer Schichtdicke von $100 \mathrm{~nm}$.

In Abbildung 6.11 ist schließlich ein REM-Bild einer ca. $400 \mathrm{~nm}$ dicken W/SiMultischicht gezeigt, die wesentlich mehr Droplets offenbart als eine W-Einzelschicht. Diese können im Wesentlichen dem Si zugeordnet werden, was anhand von EDXKonzentrationsanalysen bestätigt wurde. Der hier zu beobachtende Kontrastunterschied gibt demnach keine Auskunft über den materiellen Ursprung, sondern spricht eher für unterschiedliche Auftreffzeiten der einzelnen Droplets während der Schichtherstellung, die durch eine nachträgliche Deposition durch W oder Si bedeckt worden sind. Die ermittelte Tröpfchendichte mit 8,5 Droplets pro $100 \mathrm{~nm}$ Multischichtdicke und $1000 \mu \mathrm{m}^{2}$ Oberfläche ist verglichen mit der des $\mathrm{Ti} / \mathrm{ZrO}_{2}-$ Schichtsystems (s. Kap. 5.1.2) gering, sodass für die MLL-Herstellung immer eine dropletfreie Substratstelle gefunden werden kann. 
Die ursprüngliche Herkunft größerer und kleinerer Tröpfchen, die hier ungefähr in gleicher Anzahl vorhanden sind, wurde bereits anhand der Untersuchungen der Laserdeposition an Metallen von FÄHLER et al. in Kapitel 5.1.2 diskutiert [135]. Darüber hinaus sind die verschiedenen Tröpfchenformen des W/Si-Systems auffällig, die auf unterschiedliche Abkühlvorgänge während des Auftreffens auf das Substrat schließen lassen. Größere Tröpfchen werden beim Aufprall weit auseinandergerissen, sodass in deren Mitten beim raschen Abkühlen eine Senke entsteht. Die masseärmeren, kleineren Droplets bilden einen kegelförmigen Zustand, der möglicherweise in einem Rückstoß des noch flüssigen Tröpfchen-Anteils begründet ist.

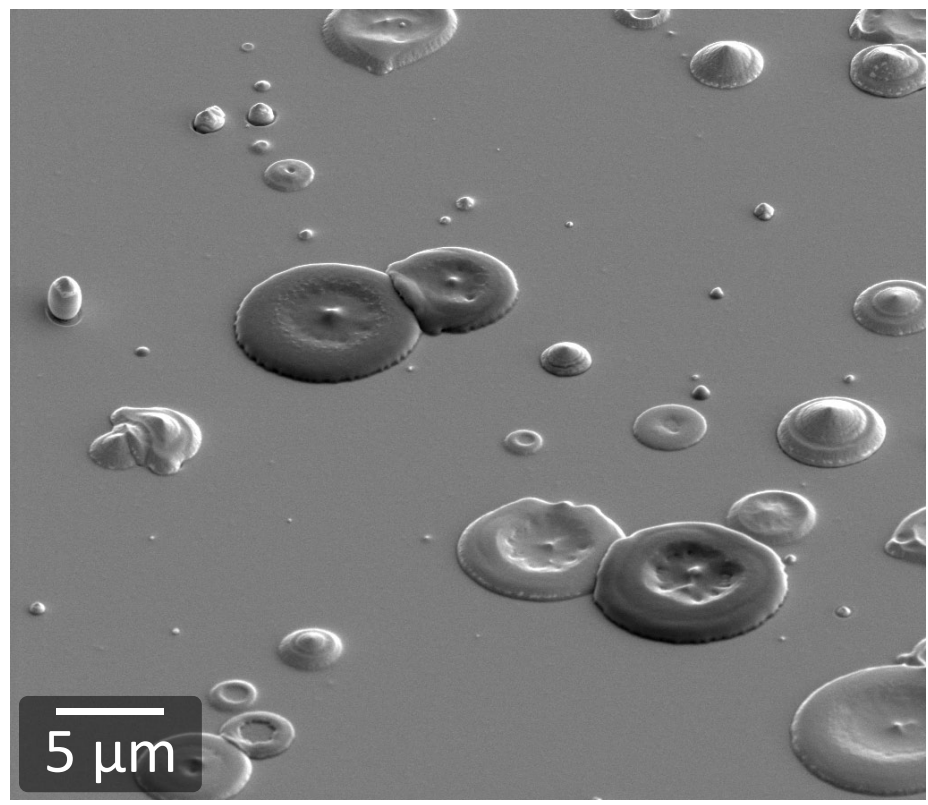

Abbildung 6.11: REM-Aufnahme einer $400 \mathrm{~nm}$ dicken W/Si-Multischicht. Die in ihrer Größe und Form unterschiedlich erstarrten Tröpfchen entstehen hauptsächlich am Si-Target.

\subsubsection{W/Si-Multischichten}

Nachdem optimale Bedingungen für die Herstellung von Einzelschichten gefunden wurden, soll im folgenden Schritt die Charakteristik des W/Si-Systems im Verbund betrachtet werden. Dabei wird vor allem deutlich, dass es für eine entsprechende Multischichtherstellung hochpräziser Schichtdicken nicht ausreicht, die Depositionsraten der Einzelkomponenten zu kennen, sondern zusätzlich die Eigenschaften der Grenzflächen von Multischichten untersucht werden müssen.

Da sich W und Si sowohl in ihrer Masse als auch in ihren erwarteten kinetischen Ionenenergien während der Laserdeposition unterscheiden, wurde untersucht, ob sich 
bei späterer Multischichtherstellung seitens einer lokalen Depositionsratenabnahme an den entsprechenden Grenzflächen ein Absputtereffekt einstellt, der dann für die weitere Herstellung berücksichtigt werden muss. Dazu wurden die deponierten Schichtdicken während einer W/Si-Multischichtherstellung mit Hilfe des in Kapitel 3.5 vorgestellten Depositionsratenmonitors in-situ mit einer hohen Schichtdickenauflösung in Abhängigkeit der Laserpulszahl aufgenommen (s. Abb. 6.12). Die dabei verwendeten Energiedichten beliefen sich auf $\rho_{\mathrm{Si}}=1,72 \mathrm{~J} / \mathrm{cm}^{2}$ und $\rho_{\mathrm{W}}=2,52 \mathrm{~J} / \mathrm{cm}^{2}$. In der Übersicht mehrerer Grenzflächen lässt sich zunächst für W und Si ein unterschiedlich großer linearer Anstieg der Schichtdicke mit steigender Pulszahl erkennen, welcher über alle hier dargestellten Einzelschichten sehr konstant ist und für unterschiedlich hohe Depositionsraten von 0,0031 nm/Puls für W und 0,012 nm/Puls für Si spricht.

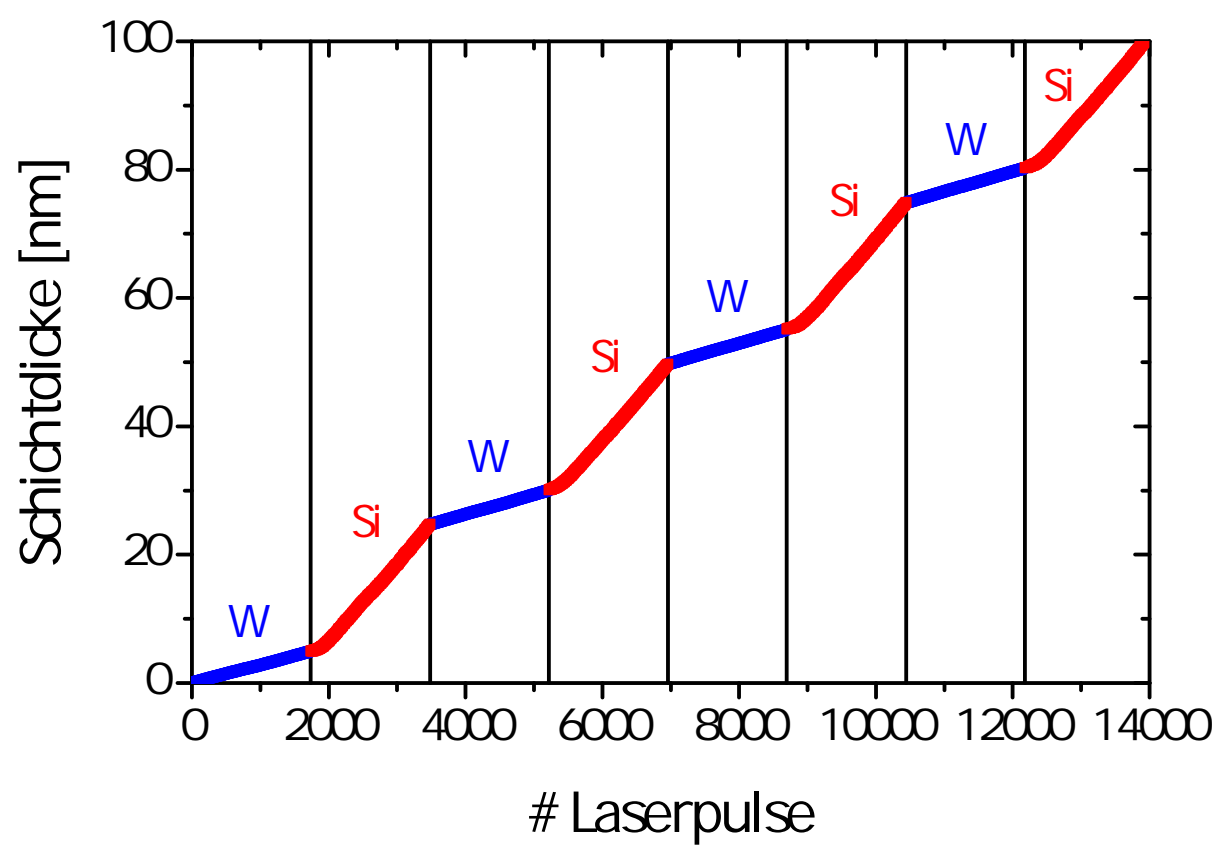

Abbildung 6.12: Schichtdickenverlauf in Abhängigkeit der Laserpulszahl eines W/SiMultischichtsystems über mehrere Grenzflächen. Verschieden große Steigungen der Einzelkomponenten sprechen für unterschiedliche Depositionsraten, die im Rahmen des hier gezeigten Messverlaufs sehr konstant sind.

Im Detail sollen beispielhaft der dritte (W/Si) und vierte ( $\mathrm{Si} / \mathrm{W})$ Grenzflächenübergang genauer betrachtet werden (s. Abb. 6.13), bei denen sich bei der Deposition von $\mathrm{Si}$ auf $\mathrm{W}$ und umgekehrt deutliche Unterschiede ergeben. Am W/SiGrenzflächenübergang stellt sich beim Si-Wachstum eine starke Abweichung vom linearen Verhalten heraus, es scheint zunächst weniger Material als bei der sich nach ca. 350 Pulsen einstellenden Gleichgewichtsrate deponiert zu werden. Da sich allerdings 
der Materialabtrag am Target bei gleicher Pulszahl nicht abrupt ändert, muss es folglich zu einem Absputtern des bereits aufgewachsenen Films durch hochenergetische SiIonen kommen, wie es auch bei dem Materialsystem Si/Au der Fall ist [191]. Ebenfalls konnte dieser Effekt bei anderen laserdeponierten metallischen System wie z. B. Cu/Ag und Fe/Ag beobachtet werden [134,192]. Der dadurch entstandene Schichtdickenverlust von 1,67 nm wird erst dann wieder in ein Gleichgewicht der Depositionsrate überführt, wenn sich die Si-Konzentration der Zwischenschicht soweit erhöht hat, dass von einer geschlossenen Si-Schicht ausgegangen werden kann [192]. Dies ist bei ungefähr $7 \mathrm{~nm}$ deponierter Schichtdicke der Fall. Aufgrund der im Vergleich zu W höheren kinetischen Energie der auftreffenden Si-Ionen (vgl. Abb. 6.7) wird bei der Deposition von Si auf W von einem größeren Abtrag als bei umgekehrter Schichtherstellung ausgegangen. Tatsächlich lässt sich beim zweiten Grenzflächenübergang (s. Abb. 6.13 rechts) anhand des linearen Verlaufs der deponierten W-Schichtdicke keine zu Beginn veränderte Depositionsrate erkennen. Dieses Verhalten war zu erwarten, zumal der Anteil an WAtomen der Plasmakeule anhand der niedrig gewählten Energiedichte sehr groß sein muss und mit kinetischen Energien zwischen 5 und $10 \mathrm{eV}$ keinen Wiederabtrag von Schichtmaterial hervorruft.
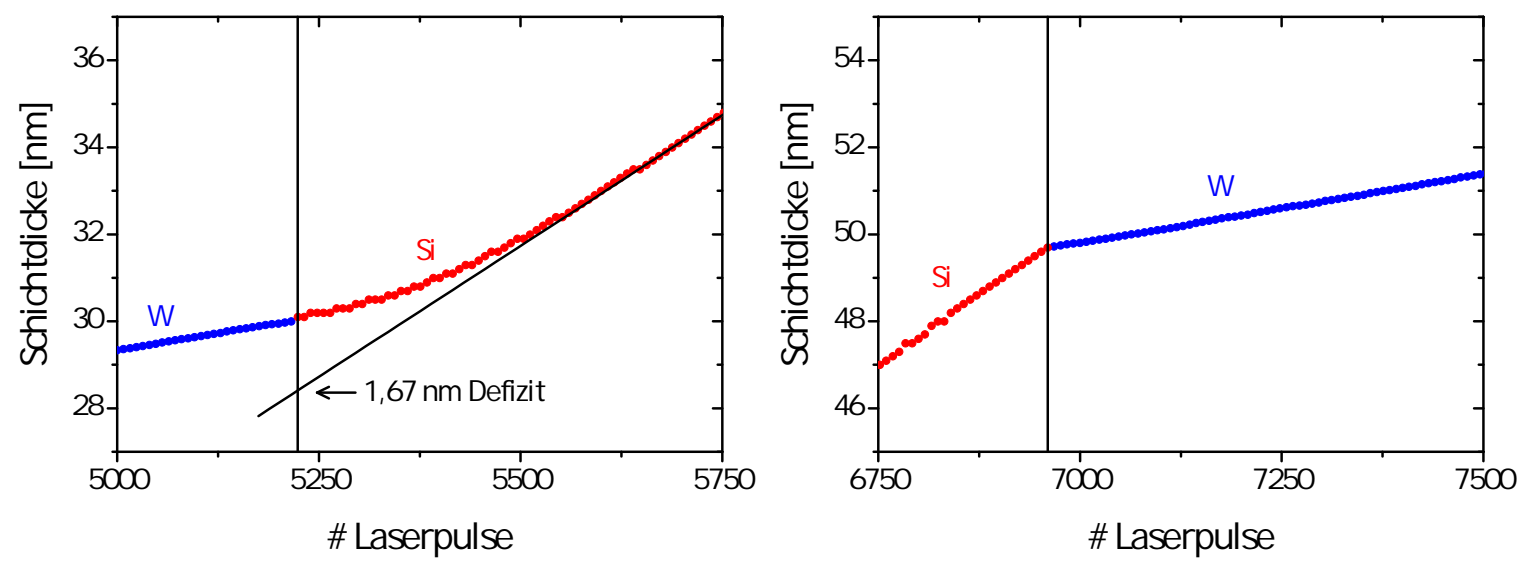

Abbildung 6.13: Untersuchungen der Depositionsraten von W und Si an den Grenzflächen des Schichtpakets. Während sich beim Übergang von W zu Si (links) ein Wiederabtrag der aufgewachsenen Schicht einstellt, zeigt sich bei der Deposition von W (rechts) ein linearer Anstieg der Schichtdicke.

Die gleichen Grenzflächenuntersuchungen wurden zusätzlich mit einer verringerten Energiedichte von $\mathrm{W}$ mit $\rho_{\mathrm{W}}=2,29 \mathrm{~J} / \mathrm{cm}^{2}$ durchgeführt, wohingegen $\rho_{\mathrm{Si}}=1,72 \mathrm{~J} / \mathrm{cm}^{2}$ konstant gehalten wurde. Während beim Si/W-Übergang wiederum kein Anzeichen eines Absputterns beobachtet werden konnte, zeigt sich bei der Deposition von Si auf W (W/Si-Übergang) in Abbildung 6.14 links ein vergleichsweise niedrigerer Wiederabtrag von Schichtmaterial mit einem Defizit von nur 0,95 nm. Im rechten Graph ist schließlich 
eine Extrapolation der zwei Schichtdickenverluste von Si bis zu der Energiedichte von $\mathrm{W} \rho_{\mathrm{W}}=2,75 \mathrm{~J} / \mathrm{cm}^{2}$ gezeigt, bei der auch die geplante MLL hergestellt wurde. Hierbei wird dann ein Verlust von etwa 2,4 nm erwartet.
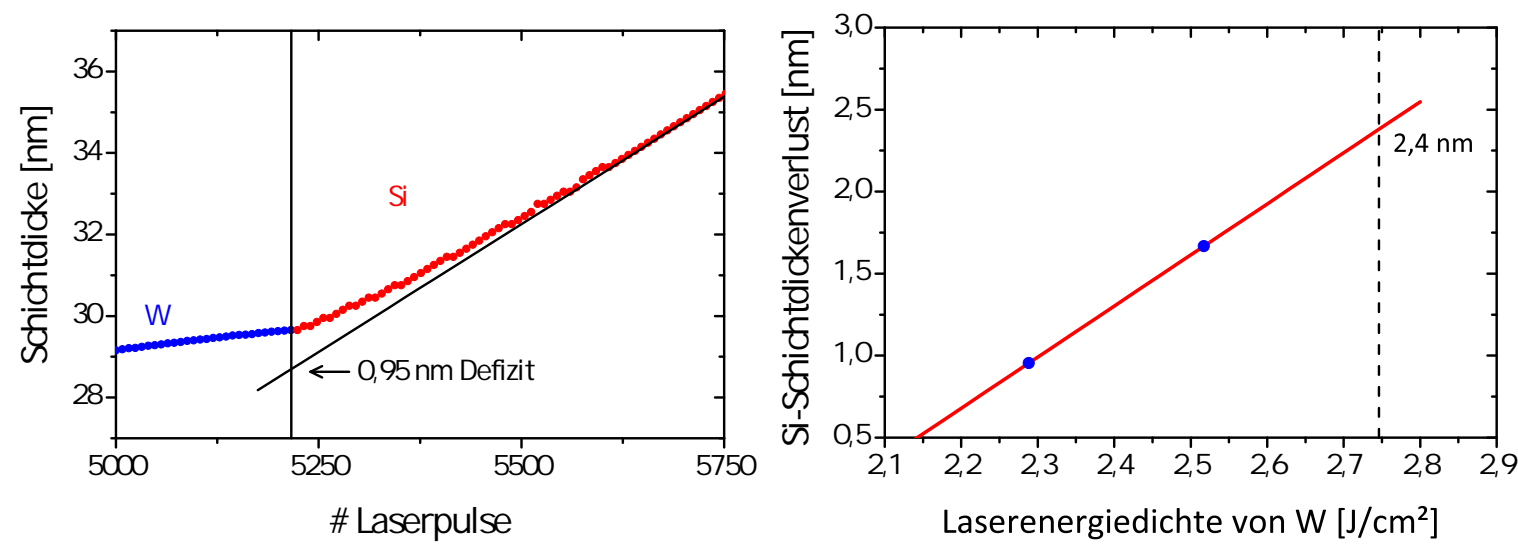

Abbildung 6.14: Bestimmung des Wiederabtrags an einer W/Si-Grenzfläche (links) bei gleicher Energiedichte von Si wie in Abbildung 6.13, aber kleinerer Energiedichte des W $\left(\rho_{\mathrm{W}}=2,86 \mathrm{~J} / \mathrm{cm}^{2}\right)$. Der extrapolierte Schichtdickenverlust bei späteren MLL-Herstellungsparametern beläuft sich auf $2,4 \mathrm{~nm}$ (rechts).

Neben der Schichtdickenanalyse wurden die auftretenden Grenzflächenphänomene zusätzlich anhand einer aperiodischen W/Si-Multischicht im TEM untersucht. Tatsächlich lässt sich an jenen W/Si-Grenzflächen, an denen ein Schichtdickenverlust gemessen wurde, ein ausgeprägter durchmischter Bereich mit einer Dicke bis zu $3 \mathrm{~nm}$ erkennen (s. Abb. 6.15). Einer thermodynamischen Ausbildung intermetallischer Phasen (vgl. W/Si-Phasendiagramm in Abb. 6.4) kann dieser Effekt allerdings nicht zugeordnet werden, da er nicht an den Si/W-Grenzflächen auftritt. Eine derart difussionsgetriebene Silizidbildung wäre höchstens im Bereich einiger Monolagen denkbar, zumal sämtliche Grenzflächen nicht atomar scharf abgebildet werden konnten.

Ferner kann der Durchmischungsbereich seitens einer für die Laserdeposition typischen Ionenimplantation begünstigt sein, zeigt sich aber auch bei gesputterten $\mathrm{W} / \mathrm{Si}$ Schichtsystemen [193]. Bei aperiodischen, von WIndT et al. hergestellten W/SiMultischichten deutet sich ebenfalls eine leicht asymmetrische Dickenverteilung der Zwischenschichten an [194]. Diese äußert sich lokal betrachtet durch etwas dickere Legierungsschichten zwischen der W/Si-Grenzfläche im Vergleich zu denen zwischen Si/W. Als Ursache für die Entstehung der Zwischenschichten wird Diffusion sowie eine unterschiedliche energetische Deposition der Einzelkomponenten für möglich gehalten. Im Fall der laserdeponierten $\mathrm{W} / \mathrm{Si}$-Multischichten ergibt sich dieses asymmetrische Verhältnis der Schichtdicken genau umgekehrt, was durch die Wahl der Energiedichten 


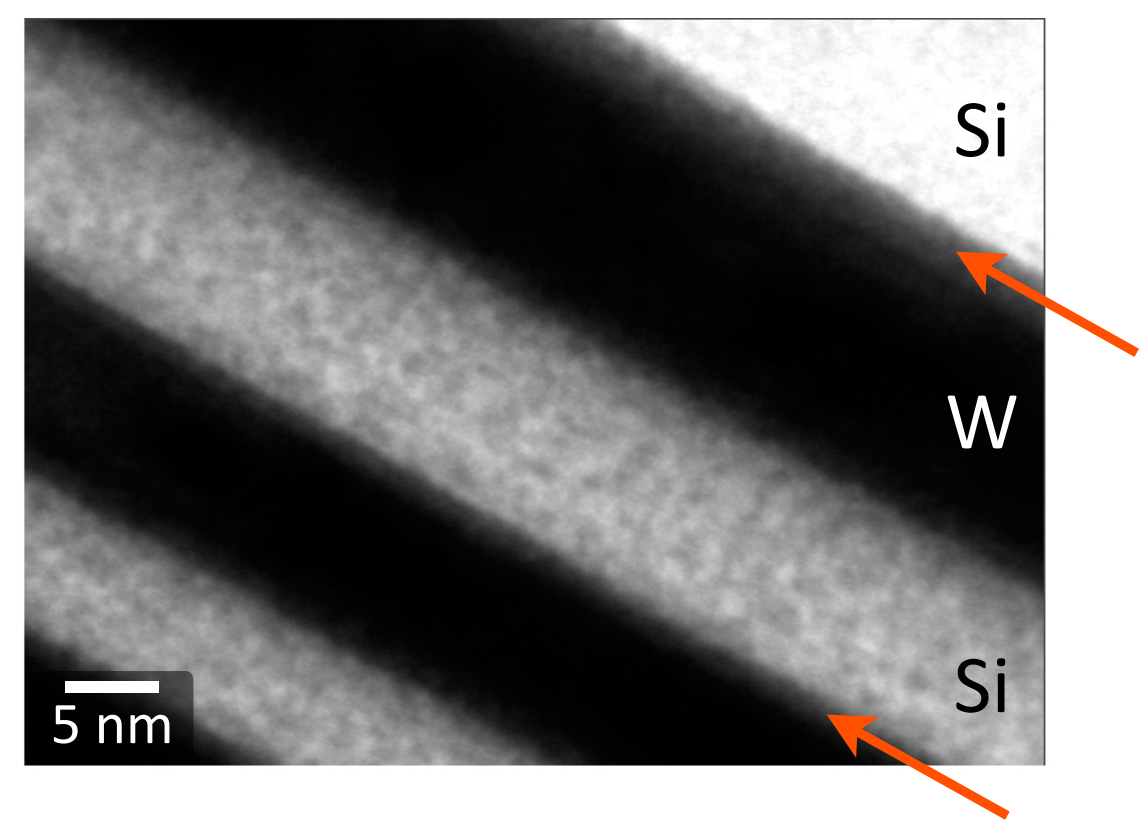

Abbildung 6.15: Querschnitt eines laserdeponierten W/Si-Multischichtsystems im TEM, bei dem das Schichtwachstum von links unten nach rechts oben erfolgt. Hauptsächlich bei der Deposition von Si auf W (durch Pfeile markiert) lässt sich an den Grenzflächen folglich einer Änderung im Materialkontrast auf einen durchmischten Bereich mit einer maximalen Dicke von $3 \mathrm{~nm}$ schließen. (Die Aufnahme wurde freundlicherweise von Felix Schlenkrich angefertigt.)

von $\mathrm{Si}$ (hoher Ionenanteil) und $\mathrm{W}$ (niedriger Ionenanteil) begründet sein kann (vgl. Abb. 6.7) und wahrscheinlich zu einer einseitigen Ionenimplantation führt.

Anhand vorausgegangener Untersuchungen lässt sich also die Existenz zweier Asymmetrien an den W/Si-Grenzflächen festhalten: zum einen merklicher Schichtdickenverlust und zum anderen eine sichtbare Durchmischung von W und Si. Dabei liegt die Vermutung nahe, dass beides durch einen hohen Ionisationsgrad von Si in einem Absputtereffekt und einer Ionenimplantation begründet ist. Dies soll im folgenden Kapitel näher untersucht werden. Der Ionenanteil von W hingegen ist dem Anschein nach fast vernachlässigbar, sodass größtenteils eine niederenergetische Deposition von Atomen auf der Schichtoberfläche stattfindet. 


\subsubsection{Theoretische Analysen auftretender Grenzflächen-Phänomene}

Zum Verständnis dieser Asymmetrien an den Grenzflächen des W/Si-Systems werden im Folgenden zunächst theoretische Betrachtungen möglicher Sputteryielde von W und Si in Abhängigkeit der Ionenenergie aufgeführt. Der Sputteryield $Y$ (Anzahl herausgeschlagener Atome pro einfallendes Ion) hängt generell vom Schichtmaterial, der Ionenenergie, der Ionenmasse sowie dem Auftreffwinkel der Ionen ab [195], welcher hier allerdings aufgrund des senkrechten Einfalls außer Acht gelassen wird. Weiterhin wird zwischen einem Eigensputteryield $Y_{\mathrm{A} \rightarrow \mathrm{A}}$, welcher den Wiederabtrag während der Schichtherstellung eines Materials A und somit die Depositionsrate im Gleichgewicht bestimmt, und dem Fremdsputteryield $Y_{\mathrm{A} \rightarrow \mathrm{B}}$ unterschieden, welcher an der Grenzfläche zwischen zwei Materialien A und B auftreten kann. Eine quantitative Abschätzung des Sputteryields von Material $\mathrm{A}\left(Z_{1}, M_{1}\right)$ auf Material $\mathrm{B}\left(Z_{2}, M_{2}\right)$ soll anhand der Berechnungen von YAmAmuRA und TAWARA erfolgen [196]:

$$
Y_{\mathrm{A} \rightarrow \mathrm{B}}=0,042 \frac{Q_{Z_{2}} \alpha_{M_{1} / M_{2}}^{*}}{U_{S}} \frac{S_{n}(E)}{1+\Gamma k_{e} \epsilon^{0,3}}\left(1-\sqrt{\frac{E_{t h}}{E}}\right)^{s} .
$$

Dabei ist $U_{S}$ die Oberflächenbindungsenergie, $S_{n}(E)$ das nukleare Bremsvermögen, $k_{e}$ der elektronische Absorptionskoeffizient, $\epsilon$ die reduzierte Energie während eines Stoßprozesses und $E_{t h}$ die Schwellenenergie des auftreffenden Ions, ab der ein Absputtern hervorgerufen wird. Das Masseverhältnis der Stoßpartner wird durch $\alpha^{*}$ berücksichtigt und $Q, \Gamma$ und $s$ sind dimensionslose materialspezifische Parameter. Die benötigte Schwellenenergie $E_{t h}$ für einen Wiederabtrag ergibt sich mit dem EnergietransferFaktor $\gamma=4 M_{1} M_{2} /\left(M_{1}+M_{2}\right)^{2} \mathrm{zu}$

$$
E_{t h}= \begin{cases}U_{S} \frac{6,7}{\gamma} & \text { für } M_{1} \geq M_{2}, \\ U_{S} \frac{1+5,7\left(M_{1} / M_{2}\right)}{\gamma} & \text { für } M_{1} \leq M_{2} .\end{cases}
$$

Wird für die Laserdeposition von Si auf W eine kinetische Energie von $E_{\mathrm{Si}}=100 \mathrm{eV}$ angenommen, so ergibt sich ein theoretischer Sputteryield von $Y_{\mathrm{Si} \rightarrow \mathrm{W}}=0,034$, d. h. dass ungefähr pro 29 auftreffende Si-Ionen ein W-Atom aus der bereits aufgewachsenen Schicht herausgeschlagen wird. Bei der Deposition von W auf Si ist schon anhand der Erkenntnisse aus den energiedichteabhängigen Depositionsratenmessungen 
(vgl. Abb. 6.7) klar geworden, dass ein Großteil der deponierten W-Teilchen niederenergetische Atome sind, welche kein Absputtern hervorrufen. Ob die wenigen $\mathrm{W}-$ Ionen mit einer abgeschätzten kinetischen Energie von $E_{\mathrm{W}}<100 \mathrm{eV}$ dennoch zu einem Wiederabtrag von Schichtmaterial führen, wurde ebenfalls mit der theoretischen Berechnung des Sputteryields überprüft. In diesem Fall ergibt sich eine Schwellenenergie von $E_{t h}=130 \mathrm{eV}$, sodass sich der Theorie zufolge an der Grenzfläche Si/W überhaupt kein Sputteryield einstellt, wie es bereits anhand des experimentellen Ergebnisses aus Abbildung 6.13 rechts vermutet wurde.

Durch SRIM-Simulationen (engl. Stopping and Range of Ions in Matter, [197]) soll nun eine detailliertere Berechnung der Sputteryielde erfolgen. Diese Monte-CarloSimulationen, mit denen die Wechselwirkung von energetischen Ionen mit amorphen Targets simuliert wird, sind jedoch keine dynamischem Prozesse, bei denen sich das Target mit zunehmender Implantation kompositionell sowie strukturell verändert. Jedes auftreffende Ion sieht vielmehr eine völlig intakte Schichtoberfläche des Ausgangsmaterials. Die Schichtoberflächen von W und Si wurden mit jeweils 100000 Si- bzw. W-Ionen mit PLD-typischen Energien von $50-150 \mathrm{eV}$ beschossen, um die Implantationstiefen der Ionen sowie die Verteilung der an Stoßprozessen beteiligten Schichtatome zu simulieren. In Abbildung 6.16 lassen sich beispielhaft für $\mathrm{Si} \rightarrow \mathrm{W}$ und für $\mathrm{W} \rightarrow \mathrm{Si}$ mit Ionenenergien von $100 \mathrm{eV}$ in den jeweils linken Graphen die zweidimensionalen Verteilungen der einfallenden Ionen (rot) sowie durch Stoßkaskaden aus ihren ursprünglichen Positionen verlagerte Schichtatome (grün) und in den rechten Graphen deren zugehörige statistische Häufigkeiten verfolgen.

Bei Betrachtung der Deposition von Si-Ionen auf W (s. Abb. 6.16 oben) lässt sich eine oberflächennahe Implantation mit einer mittleren Eindringtiefe bis zu 0,63 nm erkennen. Demnach finden sämtliche Stoßprozesse nahe der Oberfläche statt, weshalb ein Absputtern der bereits deponierten W-Schicht mit einem Sputteryield von $Y_{\mathrm{Si} \rightarrow \mathrm{W}}=0,16$ zugrunde liegt (vgl. Abb. 6.17 links). Im Fall der Deposition von $\mathrm{W}$ auf Si in Abbildung 6.16 unten fällt zuerst eine periodische, radialsymmetrische W-Ionenverteilung auf, die als Artefakt der SRIM-Simulation gedeutet werden kann. Da das Schichtmaterial als monolagiges, amorphes Material angenommen ist, können primäre Stoßprozesse nur in diskreten Abständen stattfinden. Diese Abstände finden sich auch in den Häufigkeitsverteilungen wieder, bei denen die W-Ionen aufgrund ihrer hohen Masse knapp 2,5 nm in die Schicht hinein transportiert werden. Die Wechselwirkungen der $\mathrm{W}$-Ionen in einer $\mathrm{Si}-\mathrm{Schicht}$ spielen sich demnach so tief 

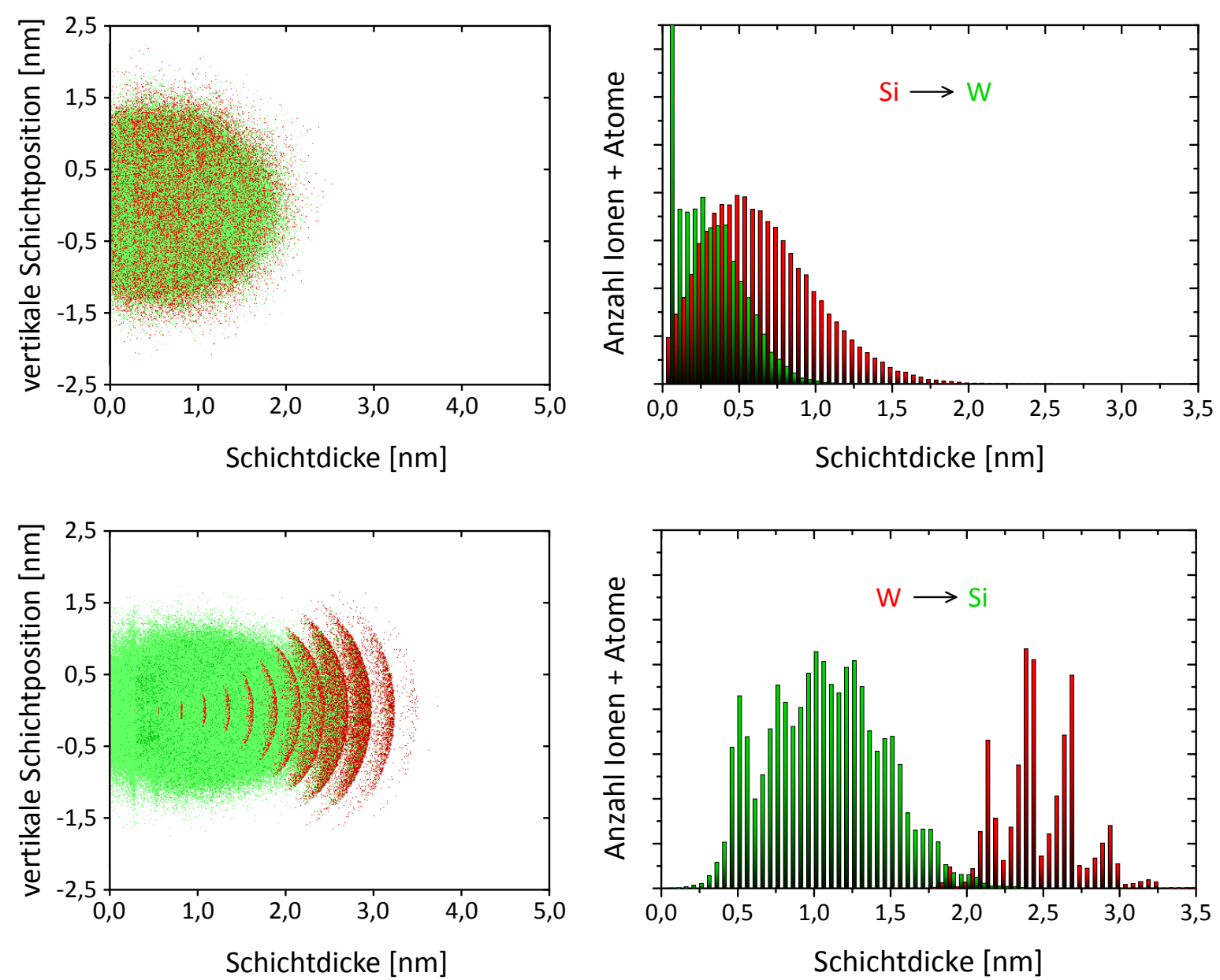

Abbildung 6.16: SRIM-Simulationen von Si auf W (oben) und umgekehrt (unten) bei einer Ionenenergie von $100 \mathrm{eV}$. Links sind die räumlichen Verteilungen der Ionen (rot) sowie der gestoßenen Schichtatome (grün) gezeigt, rechts die zugehörigen Histogramme. Aufgrund der geringen mittleren Eindringtiefe der Si-Ionen von $\bar{x}=0,63 \mathrm{~nm}$ führen oberflächennahe Stoßprozesse zu einem Wiederabtrag von Schichtmaterial. Im Fall von laserdeponierten W-Ionen ist die mittlere Eindringtiefe mit $\bar{x}=2,47 \mathrm{~nm}$ wesentlich höher, sodass an tiefer gelegenen Rückstreuprozessen beteiligte Schichtatome die Oberfläche nicht verlassen und somit keinen Sputteryield hervorrufen.

innerhalb der Schicht ab, dass sich folglich kein Wiederabtrag des bereits deponierten Si einstellt.

Graphisch lässt sich das Auftreten eines Wiederabtrags von Schichtmaterial anhand der aus den Simulationen erhaltenen integralen Sputteryielde $Y_{\mathrm{Si} \rightarrow \mathrm{W}}$ und $Y_{\mathrm{W} \rightarrow \mathrm{Si}}$ veranschaulichen. In Abbildung 6.17 sind sie dazu bei jeweils $100 \mathrm{eV}$ Ionenenergie als Funktion der Energie der gestreuten Atome aufgetragen, wobei lediglich diejenigen Atome $\mathrm{zu}$ einem Absputtern beitragen, deren übertragene Energie während eines Stoßes größer als die Oberflächenbindungsenergie $U_{S}$ des jeweiligen Schichtmaterials 
ist. Somit sind W-Atome mit einer mittleren Energie von $12,8 \mathrm{eV}$ in der Lage, die Schichtoberfläche zu verlassen, während den gestoßenen Si-Atomen im Mittel keine ausreichende Energie übertragen wurde, um abgesputtert $\mathrm{zu}$ werden (obwohl sie schwächer gebunden sind als W [196]).
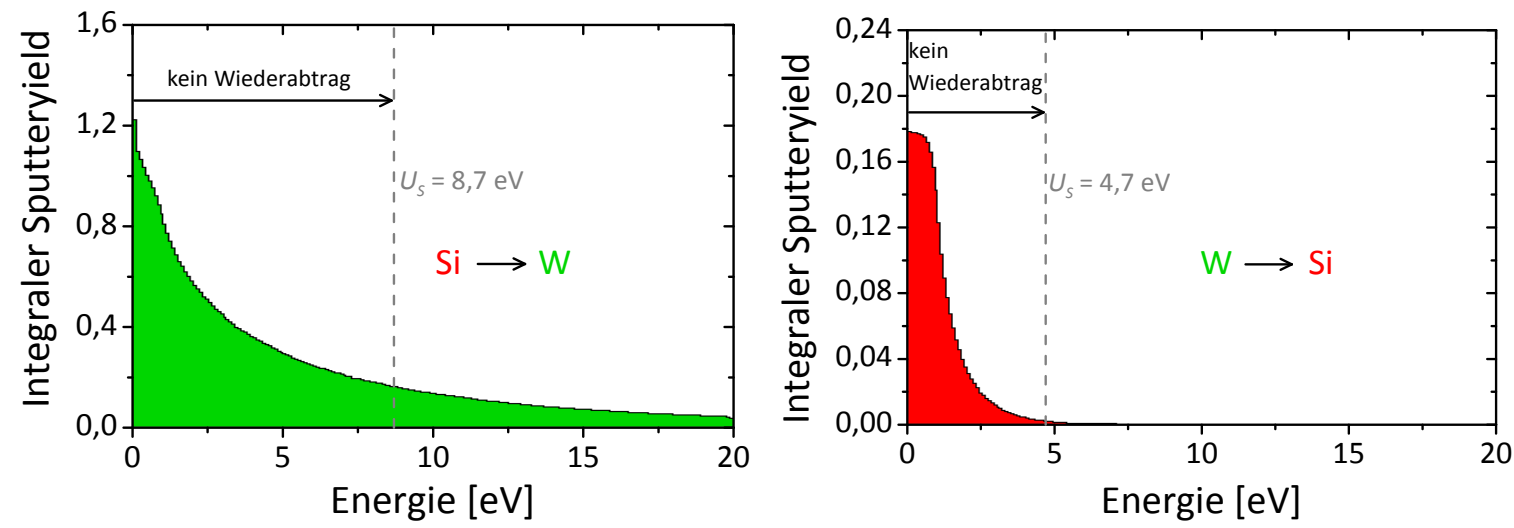

Abbildung 6.17: Integrale Sputteryielde bei Ionenenergien von $100 \mathrm{eV}$ von $\mathrm{W}$ (links) und Si (rechts) in Abhängigkeit der an gestoßene Schichtatome übertragenen Energie. Nur diejenigen Atome, die die Oberflächenbindungsenergie $U_{S}$ überwinden können, tragen zu einem effektiven Sputtern bei.

Eine Übersicht der Sputteryielde $Y$ aller Kombinationen aus Fremd- und Eigensputteryielde und den mittleren Eindringtiefen $\bar{x}$ von Si und W bei unterschiedlichen Ionenenergien ist in Tabelle 6.2 aufgeführt. Generell wurden leicht abweichende Simulationsergebnisse im Vergleich zu jenen Sputteryielden $Y_{\mathrm{Si} \rightarrow \mathrm{W}}$ und $Y_{\mathrm{W} \rightarrow \mathrm{Si}}$ festgestellt, die mit Gleichung 6.1 berechnet wurden. Es zeigen sich allerdings die gleichen Tendenzen bei steigenden Ionenenergien. Im Fall von Si lässt sich Tabelle 6.2 bei Ionenenergien von 100 und $150 \mathrm{eV}$ entnehmen, dass der Fremdsputteryield $Y_{\mathrm{Si} \rightarrow \mathrm{W}}$ größer als der Eigensputteryield $Y_{\mathrm{Si} \rightarrow \mathrm{Si}}$ ist, was experimentell auf eine lokale Änderung der Depositionsrate als Ursache für einen Schichtdickenverlust an den entsprechenden Grenzflächen bis zum Gleichgewicht hin deutet. Begründet wird der Unterschied in $Y$ durch das asymmetrische Eindringverhalten der Ionen, welches abhängig vom Schichtmaterial und der Ionenenergie sind. Si dringt bei allen aufgezeigten Energien nicht so stark in W ein als in sich selbst, sodass Stoßprozesse in W näher an der Oberfläche stattfinden und zu einem größeren Wiederabtrag von Schichtmaterial führen. Mit steigender Ionenenergie nimmt zwar auch die Eindringtiefe zu, jedoch werden gestoßenen Schichtatomen größere Energien übertragen, die benötigt werden, um die Oberfläche als gesputtertes Teilchen zu verlassen. Die Tatsache, dass sich im Gegensatz dazu bei der Deposition von W auf Si kein, bzw. ein minimaler Sputtereffekt einstellt, bestätigt die experimentell gewonnenen Erkenntnisse der schichtdickenabhängigen Grenzflächenmessungen. Hier 


\begin{tabular}{|c|c|c|c|}
\hline Ionenenergie & $50 \mathrm{eV}$ & $100 \mathrm{eV}$ & $150 \mathrm{eV}$ \\
\hline$Y_{\mathrm{Si} \rightarrow \mathrm{Si}}$ & 0,059 & 0,144 & 0,219 \\
\hline$Y_{\mathrm{Si} \rightarrow \mathrm{W}}$ & 0 & 0,157 & 0,279 \\
\hline$Y_{\mathrm{W} \rightarrow \mathrm{W}}$ & 0,019 & 0,090 & 0,168 \\
\hline$Y_{\mathrm{W} \rightarrow \mathrm{Si}}$ & 0 & 0 & 0,010 \\
\hline $\bar{x}_{\mathrm{Si} \rightarrow \mathrm{Si}} \quad[\mathrm{nm}]$ & 0,849 & 1,112 & 1,338 \\
\hline $\bar{x}_{\mathrm{Si} \rightarrow \mathrm{W}} \quad[\mathrm{nm}]$ & 0,477 & 0,630 & 0,745 \\
\hline $\bar{x}_{\mathrm{W} \rightarrow \mathrm{W}}[\mathrm{nm}]$ & 0,473 & 0,545 & 0,599 \\
\hline $\bar{x}_{\mathrm{W} \rightarrow \mathrm{Si}}[\mathrm{nm}]$ & 1,979 & 2,465 & 2,780 \\
\hline
\end{tabular}

Tabelle 6.2: Mittels SRIM simulierte Eigen- und Fremdsputteryielde sowie zugehörige Eindringtiefen von $\mathrm{Si}$ und $\mathrm{W}$ bei verschiedenen Ionenenergien. Der Masseverlust an der Si/W-Grenzfläche (vgl. Abb. 6.13 links und 6.14 links) ist dadurch begründet, dass der Eigensputteryield von Si $\left(Y_{\mathrm{Si} \rightarrow \mathrm{Si}}\right)$ im Gleichgewicht niedriger als $Y_{\mathrm{Si} \rightarrow \mathrm{W}}$ ist. $\mathrm{W}$ besitzt lediglich einen niedrigen Eigen- und einen noch kleineren Fremdsputteryield.

ist der Fremdsputteryield $Y_{\mathrm{W} \rightarrow \mathrm{Si}}$ kleiner als der Eigensputteryield $Y_{\mathrm{W} \rightarrow \mathrm{W}}$, da $\mathrm{W}$ wesentlich tiefer in Si implantiert wird als in sich selbst.

Schließlich gilt es noch die Frage zu beantworten, weshalb der Si-Schichtdickenverlust mit den unterschiedlichen Laserenergiedichten des $\mathrm{W}$ in Zusammenhang steht (vgl. Abb. 6.14 rechts). Dieser Effekt lässt sich verstehen, wenn als Konsequenz einer hochenergetischen Ionenimplantation ein starkes Verdichten des bereits deponierten Films gesehen wird. Der als „shot peening“ bezeichnete Prozess [180,181] sorgt für eine lokale Dichteerhöhung nahe der Schichtoberfläche, welche wiederum eine verringerte Eindringtiefe auftreffender Ionen nach sich zieht. Wie in Tabelle 6.3 zusammengefasst wurde, führen jene oberflächennäheren Implantationen bei 1-3\% dichter angenommenen W-Schichten zu einer leichten Erhöhung der Sputteryielde $Y_{\mathrm{Si} \rightarrow \mathrm{W}}$ und damit zu größeren W-Schichtdickenverlusten mit zunehmender Laserenergiedichte.

Wie sich das unterschiedliche Eindringverhalten von Targetionen in W oder Si im Detail vollzieht, wurde mit dynamischen Monte-Carlo-Simulationen mittels TRIDYN untersucht. Dieses Programm ermöglicht Berechnungen von Konzentrationsprofilen sowie Veränderungen der Sputteryielde für eine dynamisch aufwachsende Schicht als Funktion der Bestrahlungsdosis durch Ionen [198]. Dabei werden kompositionelle Änderungen bzgl. einer Ionenimplantation sowie einer Verlagerung gestoßener Schichtatome durch ballistischen Transport berücksichtigt. Als Parametereingabe wurde eine Bestrahlungsdosis durch Si-Ionen von $1 \cdot 10^{17} \mathrm{~cm}^{-2}$ angenommen, was einer 


\begin{tabular}{lccc}
\hline \multicolumn{1}{c}{ Ionenenergie } & $50 \mathrm{eV}$ & $100 \mathrm{eV}$ & $150 \mathrm{eV}$ \\
\hline$Y_{\mathrm{Si} \rightarrow \mathrm{W}}$ & 0 & 0,157 & 0,279 \\
$Y_{\mathrm{Si} \rightarrow \mathrm{W}(\rho+1 \%)}$ & 0 & 0,160 & 0,283 \\
$Y_{\mathrm{Si} \rightarrow \mathrm{W}(\rho+3 \%)}$ & 0 & 0,162 & 0,287 \\
$\bar{x}_{\mathrm{Si} \rightarrow \mathrm{W}} \quad[\mathrm{nm}]$ & 0,477 & 0,630 & 0,745 \\
$\bar{x}_{\mathrm{Si} \rightarrow \mathrm{W}(\rho+1 \%)}[\mathrm{nm}]$ & 0,473 & 0,624 & 0,738 \\
$\bar{x}_{\mathrm{Si} \rightarrow \mathrm{W}(\rho+3 \%)}[\mathrm{nm}]$ & 0,465 & 0,612 & 0,727 \\
\hline
\end{tabular}

Tabelle 6.3: Mittels SRIM simulierte Sputteryielde $Y$ und mittlere Eindringtiefen $\bar{x}$ bei der Deposition von Si auf W in Abhängigkeit verschiedener Ionenenergien. Mit zunehmender W-Dichte steigt der Sputteryield in Folge einer geringeren Ioneneindringtiefe leicht an.

ungefähren Schichtdicke von $20 \mathrm{~nm}$ entspricht. Für W-Ionen wurde zum Vergleich mit experimentellen Daten eine Dosis von $0,35 \cdot 10^{17} \mathrm{~cm}^{-2}(\simeq 5,5 \mathrm{~nm})$ gewählt. Weitere Fitparameter sind die Ionenenergie, der Anteil an energetischen Ionen der gesamten Bestrahlung sowie als Bindungsenergien der beteiligten Materialien ( $\mathrm{Si}-\mathrm{Si}, \mathrm{Si}-\mathrm{W}$, W-W) deklarierte Werte, die zur Anpassung von Simulation an experimentelle Daten variiert werden können.

Durch Umrechnung der Bestrahlungsdosis in eine Laserpulszahl konnten die Schichtdickenverläufe aus Abbildung 6.13 exakt simuliert werden. Die experimentellen Daten des Wachstums von Si auf W wurden mit einer angenommenen Si-Ionenenergie von $100 \mathrm{eV}$ und einem Ionenanteil der Plasmakeule von 100\% angepasst (s. Abb. 6.18 links). Der hohe Ionenanteil ist wiederum durch die Wahl der Energiedichte für $\mathrm{Si}$ im gesättigten Bereich der Depositionsrate (vgl. Abb. 6.7) vertretbar. Der genaue Verlauf der Schichtdicke nahe der W/Si-Grenzfläche mit auftretendem Schichtdickenverlust wird im folgenden Abschnitt anhand lokaler Konzentrationsprofile detailliert beschrieben. Bei der Deposition von W auf Si in Abbildung 6.18 rechts ergab die Übereinstimmung von Messung und Simulation, dass aufgrund der niedrigeren mittleren kinetischen Energie der W-Ionen (vgl. Abb. 6.7) und einer resultierenden hohen Rekombinationsrate lediglich 5\% der auftreffenden $\mathrm{W}$-Teilchen Ionen mit einer Energie von $100 \mathrm{eV}$ sind. Diese rufen wegen der hohen Eindringtiefe in Si (vgl. Abb. 6.16 unten) jedoch kein Absputtern an der Grenzfläche hervor, weshalb sich hier im Gegensatz zur Deposition von Si auf W kein messbares Schichtdickendefizit einstellt. Der wesentliche Plasmabestandteil rekombinierter W-Atome $\left(E_{k i n}=5-10 \mathrm{eV}\right)$ wird zudem nicht in die vorhandene Si-Schicht implantiert, sondern auf der Oberfläche deponiert. 

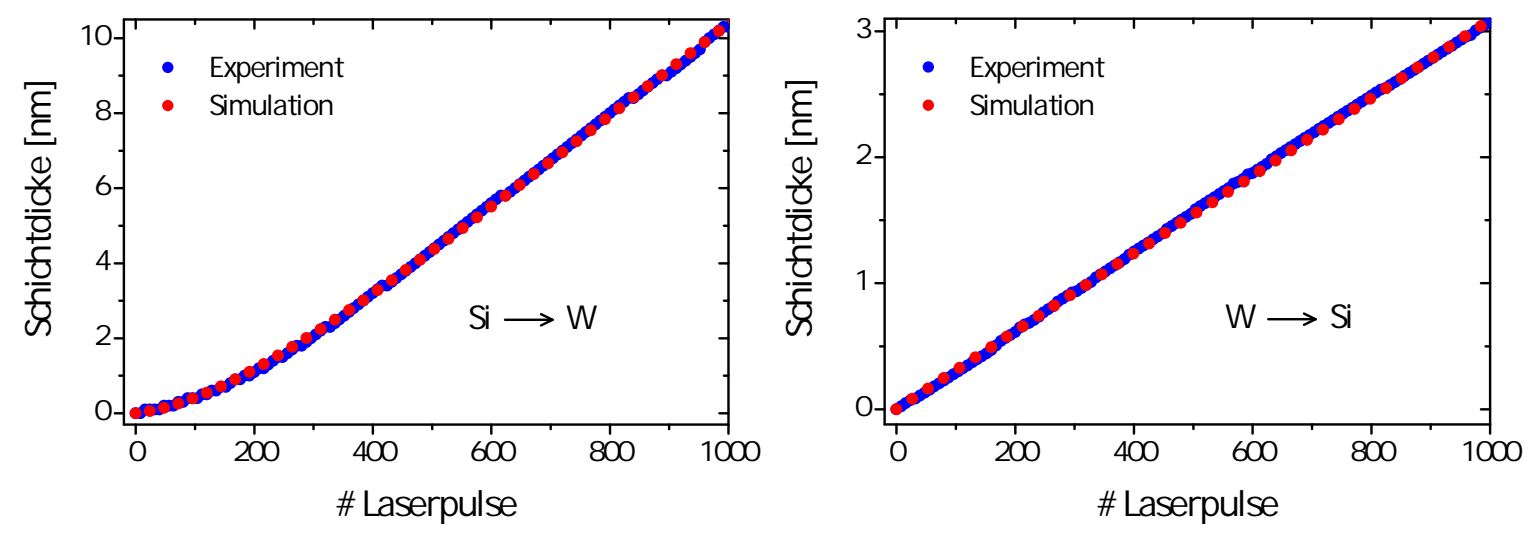

Abbildung 6.18: Vergleich von Experiment und dynamischer Simulation der Si-Deposition auf W (links) und umgekehrt (rechts) in Abhängigkeit der Laserpulszahl. Beim Wachstum von Si auf W wurden 100\% des Laserplasmas als Si-Ionen und bei der Deposition von W auf Si nur 5\% W-Ionen mit einer kinetischen Energie von $100 \mathrm{eV}$ angenommen.

Der auftretende Schichtdickenverlust bei der Si-Deposition auf W lässt sich schließlich anhand momentaner Konzentrationsprofile von W erklären, die in Abbildung 6.19 in Abhängigkeit der Schichtposition dargestellt sind. Dabei bezeichnet die Nullposition die Oberfläche der W-Schicht vor der simulierten Bestrahlung durch Si-Ionen. Bereits bei der anfänglichen Deposition von ca. $0,3 \mathrm{~nm}$ Si zeigt sich eine Implantation in die $\mathrm{W}$-Schicht mit einer mittleren Eindringtiefe von $\bar{x}_{\mathrm{Si} \rightarrow \mathrm{W}}=0,6 \mathrm{~nm}$. Dieser Wert entspricht der Senke der W-Konzentration und wurde ebenfalls anhand von SRIM-Simulationen herausgefunden. Der eigentliche Wachstumsvorgang erfolgt somit nicht an der Oberfläche, sondern einige Monolagen darunter, wie es auch bei der ionenstrahlunterstützten Sputterdeposition am Beispiel von W/C-Schichten der Fall ist [199]. Dieser bei PLD als „subsurface growth mode“ bezeichnete Effekt [134] bewirkt hier ein Ionenmischen von Si und $\mathrm{W}$ sowie ein Teilchentransport von $\mathrm{W}$ an die momentane Schichtoberfläche, wo dessen Konzentration höher als bei der mittleren Implantationstiefe des Si ist.

Durch weitere Bestrahlung mit Si-Ionen und somit fortschreitender Ionenimplantation wird der oberflächennahe Anteil an W-Atomen jeweils nach vorne geschoben, wobei der W-Schichtdickenbereich unter Abnahme der Konzentration immer dünner im Bereich weniger Atomlagen wird. Hierbei stellt sich solange ein verringertes Schichtwachstum ein, bis die $\mathrm{W}$-Konzentration an der Oberfläche komplett verarmt und der Sputteryield von Si dem des reinen Materials entspricht. Dies äußert sich in Abbildung 6.19 durch eine verringerte Schichtdicke (zu sehen an der Schichtposition) im Vergleich zur jeweils deponierten Si-Äquivalentschichtdicke der gezeigten Bestrah- 


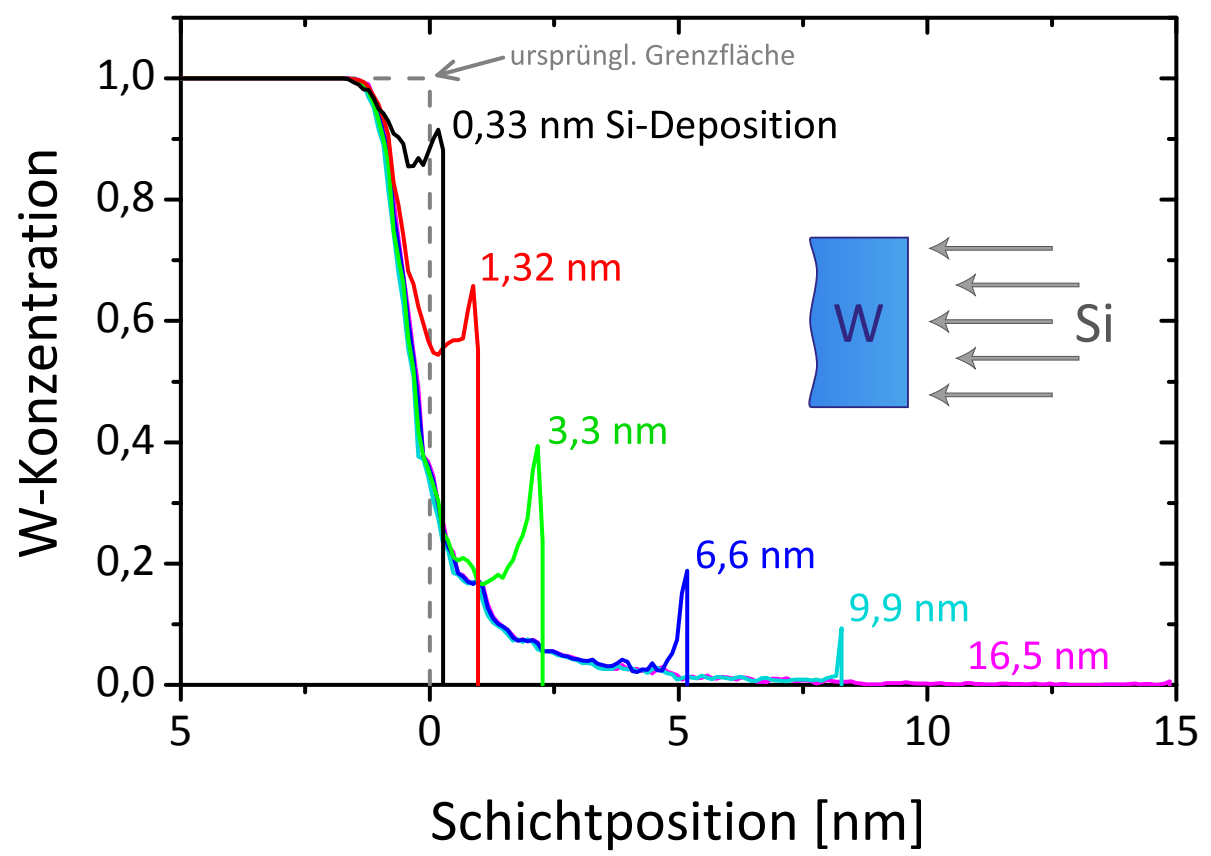

Abbildung 6.19: Momentane Konzentrationsprofile von W mit zunehmender Si-Deposition. Das hauptsächliche Wachstum des Si innerhalb der W-Schicht (Minima der W-Profile) führt zu einem Transport von W-Atomen an die Oberfläche und dies wiederum zu einer Erhöhung des Sputteryields aufgrund der Verlagerung von Stoßkaskaden in Oberflächennähe.

lungsdosen. Der Abfall der W-Konzentration im Gleichgewichtszustand (16,5 nm SiDeposition) zeigt einen etwas stärkeren als exponentiellen Verlauf, zumal W nicht nur an die Oberfläche transportiert, sondern auch wieder abgetragen wird.

Während die Schichtoberfläche noch ausreichend mit W dotiert ist, findet im Bereich maximaler Konzentration schwerer W-Atome der größte Energieverlust auftreffender Si-Ionen statt. Dies führt zu einem erhöhten Bremsvermögen und folglich zu einer Verdichtung von Stoßkaskaden nahe der Oberfläche bzw. zu einem erhöhten Fluss von rückgestreuten Teilchen des leichten Si. Als Folgerung des PLD-typischen Schichtwachstums unterhalb der Oberfläche findet deshalb eine Verstärkung des Sputteryields (engl. sputter yield amplification, SYA) statt, welche generell immer dann auftritt, wenn Ionen eines leichten Elements auf ein schweres deponiert werden [200,201]. Durch die Wahl der Ionenenergie während einer Multischichtherstellung mittels PLD lässt sich somit eine gezielte Steuerung der Grenzflächeneigenschaften vornehmen. Bei der Sputterdeposition wird der SYA-Effekt sogar dazu ausgenutzt, die Ablationsrate eines Al-Targets durch eine minimale W-Dotierung um bis zu $80 \%$ zu steigern $[202,203]$.

Erfreulicherweise nimmt das durch Ionenmischen entstehende Konzentrationsprofil an der W/Si-Grenzfläche theoretisch einen positiven Einfluss auf die Effizienzeigen- 
schaften einer MLL. Der nahezu exponentielle Abfall der W-Konzentration stellt wie in Abbildung 6.20 gezeigt eine grobe Näherung an ein ideales Sägezahnprofil der Elektronendichte zweier Zonenmaterialien dar, um einen sukzessiven Phasenschub und somit eine erhebliche Effizienzsteigerung zu erzielen (Multilevel-MLL, vgl. Kap. 2.3.1).

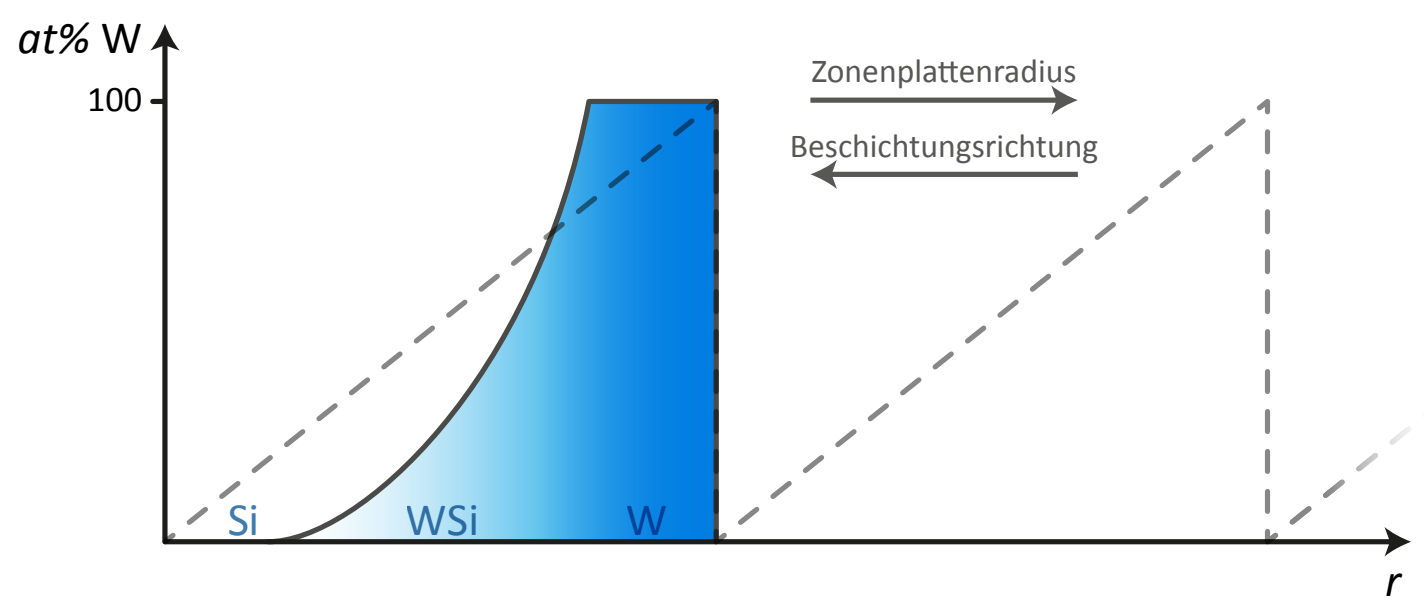

Abbildung 6.20: Annäherung an ein ideales Sägezahnprofil einer Multilevel-MLL. Dank Ionenmischen an der $\mathrm{W} / \mathrm{Si}$-Grenzfläche wird sich der Theorie zufolge die Effizienz der MLL durch einen verbesserten Phasenschub aufgrund sukzessiv steigender Elektronendichte erhöhen.

\subsection{MLL-Schichtherstellung mittels PLD}

Mit Hilfe der vorangegangenen Kenntnisse über das Depositionsverhalten des $\mathrm{W} / \mathrm{Si}$ Materialsystems konnte die eigentliche Schichtherstellung für eine MLL vorbereitet werden. Erfahrungsgemäß ist es dabei von besonderer Wichtigkeit, noch einmal kurz vor der MLL-Herstellung eine Serie von Messungen der Depositionsraten von W/SiMultischichten vorzunehmen, um den aktuellen energiedichte- und somit laserabhängigen Schichtdickenverlust an den Grenzflächen zu ermitteln. Besonders geeignet ist hierbei die Herstellung mehrerer periodischer Schichtpakete mit möglichst gleicher Einzelschichtdicke (Verhältnis $\Gamma=0,5$ ), da ein Großteil der äußeren MLL-Zonen aufgrund sehr geringer Schichtdickenänderung als nahezu periodisch angesehen werden kann. Um dabei eine Aussage über die zeitliche Stabilität der einzelnen Raten treffen zu können, wurden die Pulszahlen der Einzelschichten verschiedener Proben teilweise konstant gehalten aber auch variiert. Darüber hinaus wurde durch die Schichtdickenvariation einzelner Proben überprüft, ob überhaupt sehr dünne für die MLL benötigten Multischichten mit einer intakten Grenzflächenstruktur hergestellt werden können. 
Da diese Information mit den im letzten Abschnitt durchgeführten Grenzflächenmessungen kaum zugänglich ist, wurden röntgenreflektometrische Untersuchungen (XRR) vorgenommen. Außerdem lassen sich trotz der hohen Sensitivität des Depositionsratenmonitors auf Schichtdickenänderungen absolute Doppel- und Einzelschichtdicken wesentlich genauer anhand der XRR-Spektren bestimmen. Nebenbei lassen sich anhand dieser Methode auch Aussagen über Rauigkeiten der unterschiedlichen Grenzflächen treffen.

Die Herstellung dieser Proben erfolgte mit einer mittigen Ausrichtung der Plasmakeulen auf das Si-Substrat, da die laterale Homogenität für die Fabrikation von Transmissionsoptiken im Mikrometerbereich ausreichend gegeben ist. Für größere Substratabmessungen, wie sie für die Röntgenanalyse benötigt wird, zeigt sich sehr wohl eine laterale Schichtdickenvariation, die bei den gemessenen XRR-Spektren in Form von Intensitätsverlusten sowie teilweiser Verbreiterung der Bragg-Peaks auftritt. Darüber hinaus wurde in den Simulationen nicht das Vorhandensein von durchmischten WSi-Zwischenschichten berücksichtigt, da diese Bildung zu dem Zeitpunkt der durchgeführten Experimente noch nicht anhand von TEM-Untersuchungen bestätigt wurde. Trotz der deutlichen Abweichung von Simulation und Messung, wie sie beispielhaft in Abbildung 6.21 für eine W/Si-Multischicht mit 5 Doppelschichten der Periode von 10,35 nm sichtbar wird, konnten die genauen Einzelschichtdicken unter Berücksichtigung entsprechender Rauigkeiten durch Anfitten der Reflexionskante sowie der Anpassung der Reflektivitätsverhältnisse einzelner Bragg-Paare extrahiert werden. Die Einzelschichtdicken von $\mathrm{W}$ und Si wurden zu $d_{\mathrm{W}}=5,09 \mathrm{~nm}$ und $d_{\mathrm{Si}}=5,26 \mathrm{~nm}$ bei einem Schichtdickenverhältnis von $\Gamma=0,508$ bestimmt.

Da sich die Einzelschichtdicken der kleinsten Zonen der konzipierten MLL auf nur 1,6 nm belaufen, wurden zusätzlich extrem dünne $\mathrm{W} / \mathrm{Si}-$ Multischichten hergestellt und röntgenreflektometrischen Messungen unterzogen. In Abbildung 6.22 ist dazu die in diesem Zusammenhang dünnste laserdeponierte 10x(W/Si)-Probe mit einer Periode von 3,45 nm gezeigt. Trotz der sehr geringen simulierten Si-Schichtdicke von nur 0,35 nm zeigt das Spektrum ausgeprägte Bragg-Peaks, die für eine intakte Periodizität der Multischichtstruktur sprechen.

Die Ergebnisse zu den Einzelschichtdicken aller vorbereitenden XRR-Messungen in Abhängigkeit ihrer zugehörigen Laserpulse sind in Abbildung 6.23 aufgetragen. Eine lineare Anpassung für beide Materialien zeigt, dass die geringere W-Depositionsrate mit 0,0031 nm/Puls aufgrund der geringeren Schwankung etwas stabiler zu sein scheint als diejenige für Si mit 0,0093 nm/Puls. Erneut macht das Schichtdickendefizit bei 


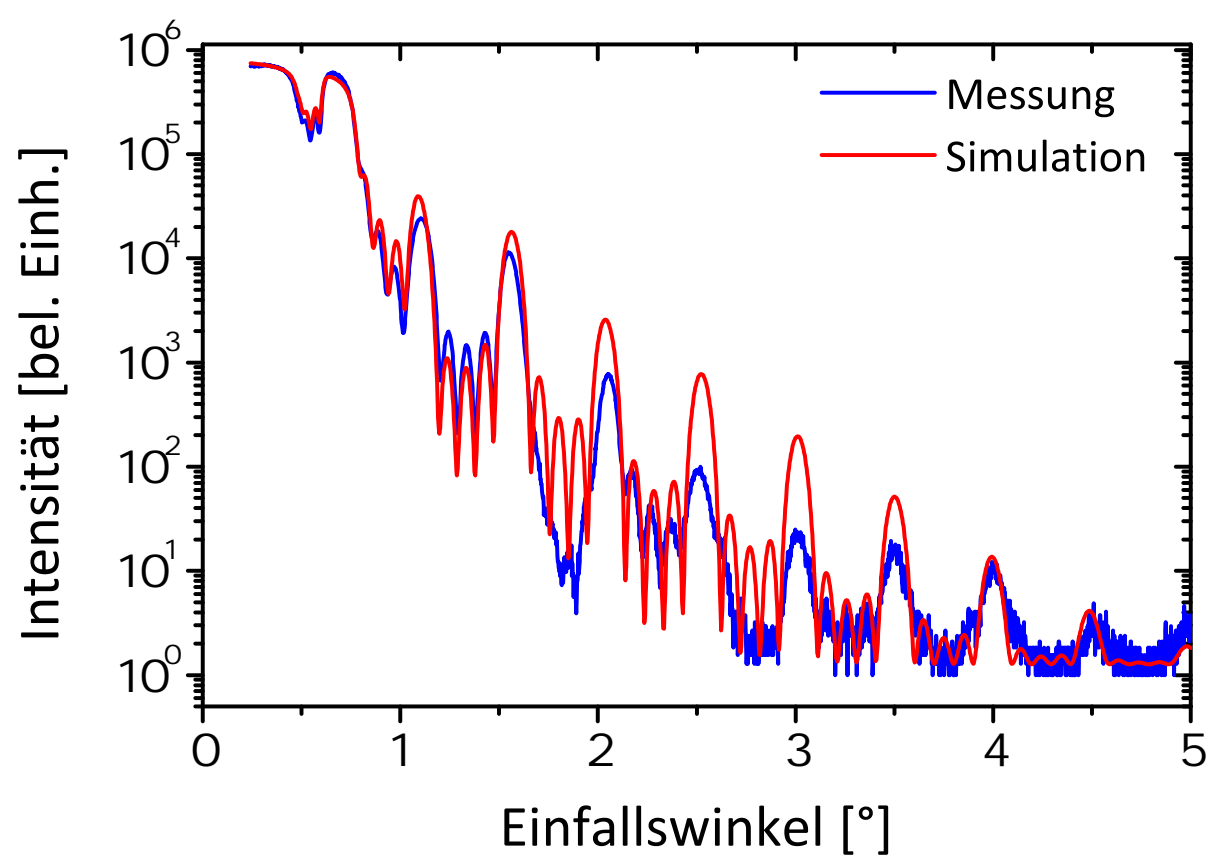

Abbildung 6.21: Als Vorbereitung für eine MLL-Herstellung benötigte XRR-Messung und Simulation einer periodischen $5 \mathrm{x}(\mathrm{W} / \mathrm{Si})-$ Multischicht zur genauen Bestimmung der Depositionsraten (s. Text).

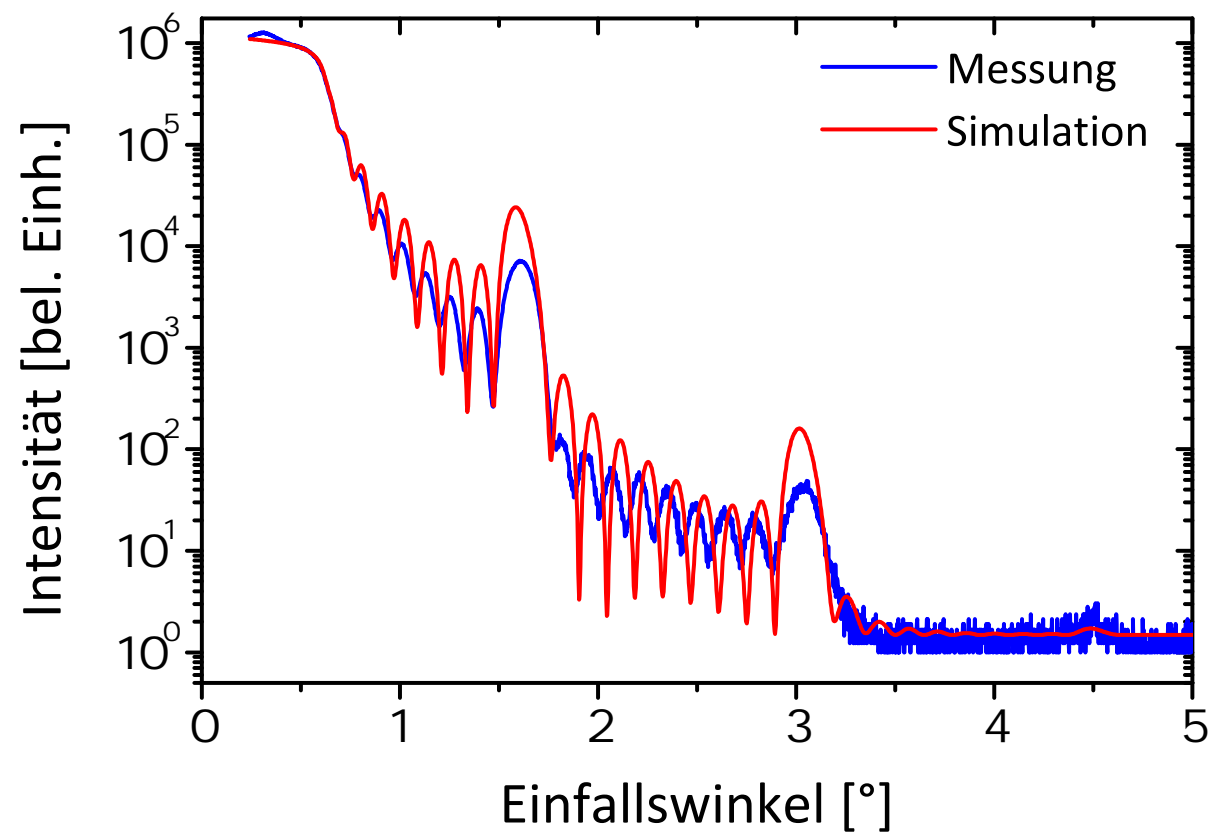

Abbildung 6.22: XRR-Spektrum eines periodischen W/Si-Schichtpakets mit 3,45 nm Doppelschichtdicke. Selbst bei sehr dünnen Einzelschichten $\left(d_{\mathrm{Si}}=0,35 \mathrm{~nm}\right)$ zeigt sich eine klare Periodizität einer intakten Multischichtstruktur.

Si von 2,5 nm das Vorhandensein eines Sputtereffekts deutlich, der bei der späteren Herstellung der MLL mit einer entsprechenden Addition auf jede Si-Einzelschichtdicke 
berücksichtigt werden muss. Verglichen mit den Ergebnissen der durchgeführten Grenzflächenmessungen (s. Abb. 6.14 rechts) lässt sich der Si-Schichtdickenverlust ungefähr der Extrapolation auf diejenige Energiedichte des $\mathrm{W}$ zuordnen, die zur Multischichtherstellung für die MLL gewählt wurde. Die einzelnen Daten sind nochmals in Abbildung 6.24 zusammengefasst. Bei W hingegen ergibt sich aufgrund des y-Achsenabschnitts bei Null kein Wiederabtrag des Schichtmaterials durch das leichtere $\mathrm{Si}$, wie vorn bereits theoretisch berechnet und durch die Depositionsratenmessungen an der Grenzfläche (vgl. Abb. 6.13 rechts) bestätigt wurde.

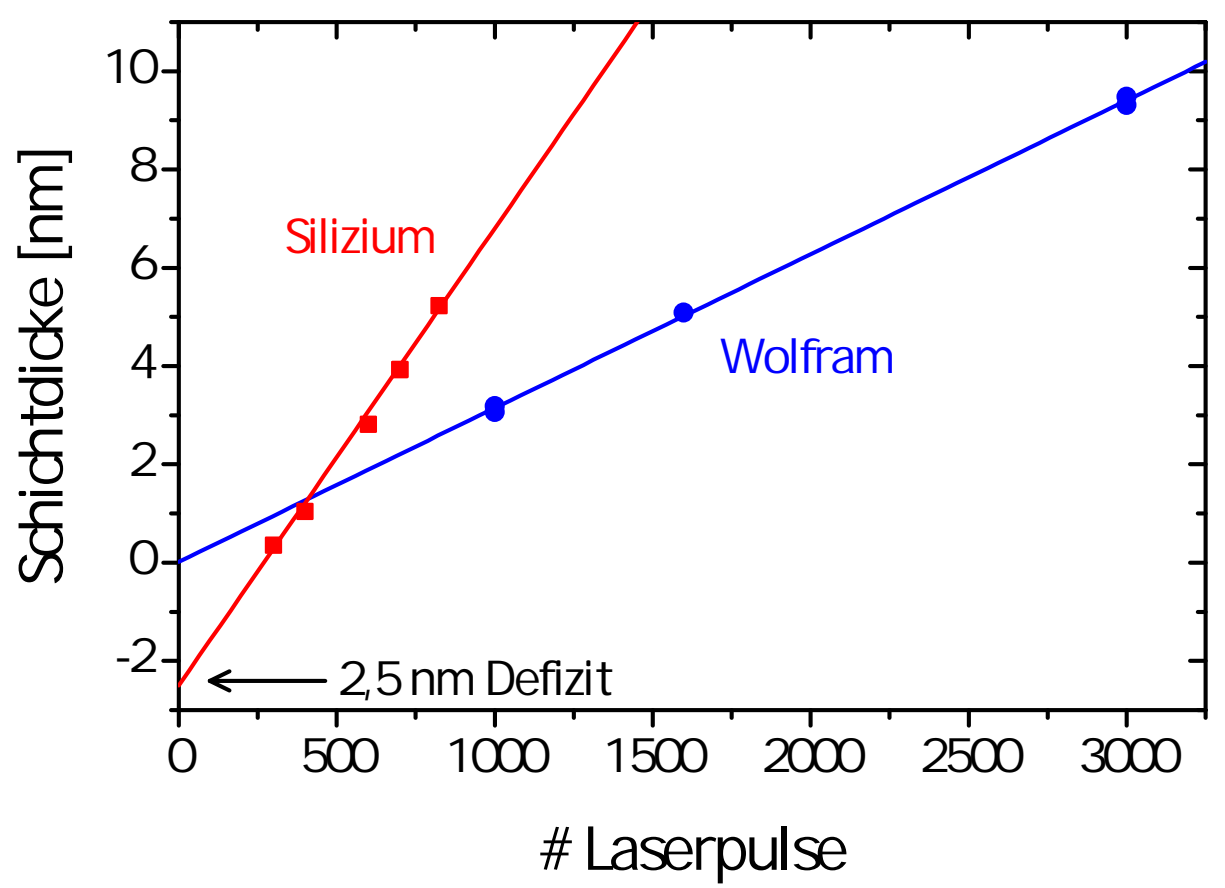

Abbildung 6.23: Anhand der XRR-Analysen erhaltene Einzelschichtdicken periodischer W/SiSchichtpakete in Abhängigkeit ihrer abgegebenen Laserpulse. Das auftretende Defizit von Si mit 2,5 nm muss in die spätere Schichtdickenberechnung für die geplante MLL miteinbezogen werden.

Mit der genauen Kenntnis der aktuellen Depositionsraten und des Wiederabtrags an der W/Si-Grenzfläche konnten nun die geeigneten Parameter ausgewählt und die 122 aperiodischen Einzelschichten der MLL für harte Röntgenstrahlung nach den in Kapitel 6.1 vorgegebenen Designparametern hergestellt werden. Die Deposition des $400 \mathrm{~nm}$ dicken Schichtpakets auf einem $1 \mu \mathrm{m}$ dicken $\mathrm{Si}_{3} \mathrm{~N}_{4}$-Substrat mit insgesamt 99000 benötigten Laserpulsen begann dabei mit der dünnsten W-Schicht, um die auflösungsbestimmenden Zonen möglichst präzise herstellen zu können. In Abbildung 6.25 ist eine Querschnittsübersicht der mit den nach dem Zonenplattenbildungsgesetz definierten Einzelschichten im TEM aufgezeigt, bei der die hellen Schichten dem amorph gewachsenen Si und die dunkleren dem W angehören. Letzteres lässt unterhalb 


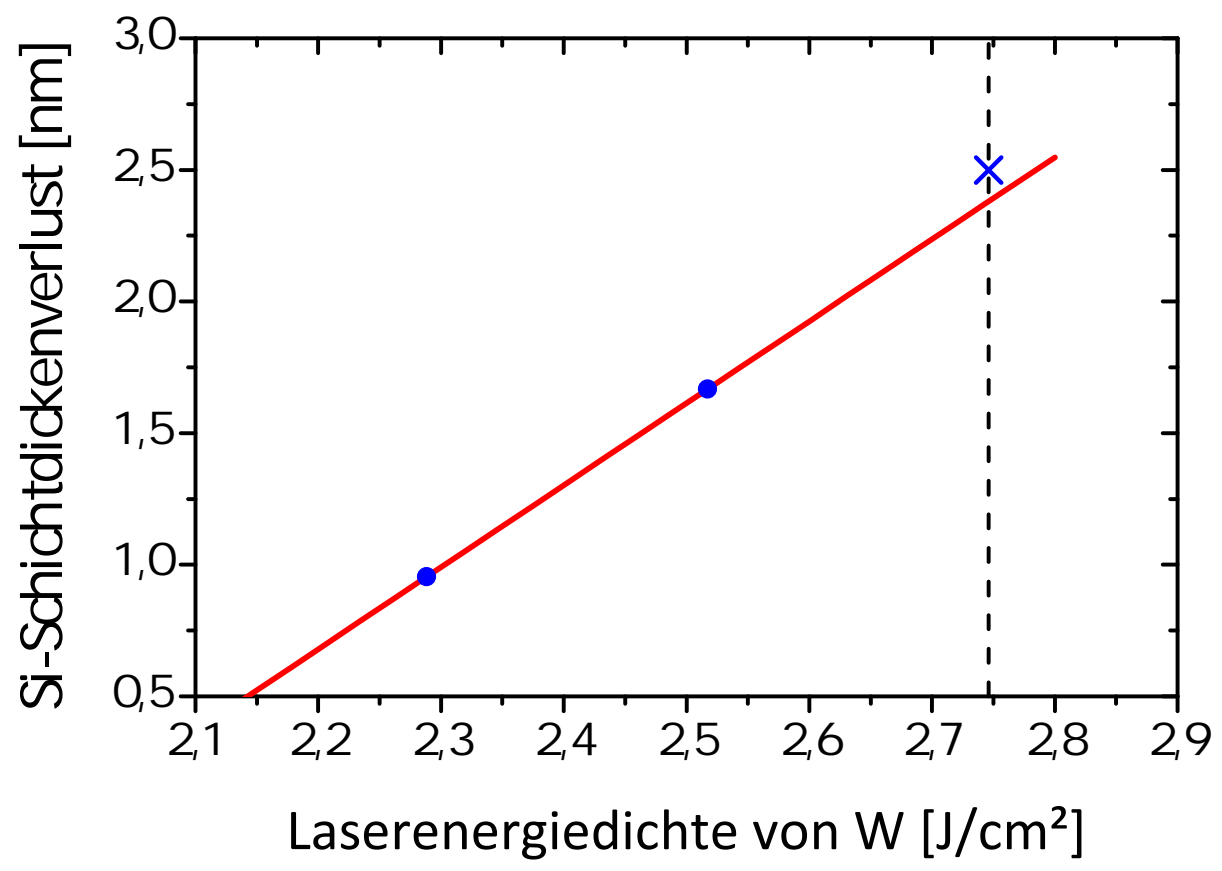

Abbildung 6.24: Die anhand der Untersuchungen der Grenzflächen ermittelten Schichtdickenverluste (vgl. Abb. 6.14 rechts) wurden um den Messwert aus den vorbereitenden XRR-Analysen (vgl. Abb. 6.23) zur MLL-Herstellung ergänzt. Erwarteter und gemessener Verlust stimmen dabei relativ gut überein.

einer Schichtdicke von ca. $8 \mathrm{~nm}$ einen strukturellen Wechsel von kristalliner zu amorpher Phase erkennen, was anhand der Analyse lokaler Beugungsbilder beobachtet wurde. Diese schichtdickenabhängige Amorphisierung des W zeigt sich ebenfalls bei gesputterten W/Si-Multischichten und wird durch einen möglichen kompositionellen Einfluss des amorphen Si durch Ionenmischen oder Diffusion erklärt [194]. Ein struktureller Übergang von amorpher zu kristalliner Phase wurde auch bei laserdeponiertem Fe auf Zr bei einer Schichtdicke von $7 \mathrm{~nm}$ festgestellt [191]. Begründet wird dies durch eine Grenzflächendurchmischung aufgrund der Deposition hochenergetischer Ionen in Kombination mit einer attraktiven Wechselwirkung, welche in diesem System einen Einfluss auf das Schichtwachstum nimmt. Ebenso typisch für die gepulste Laserdeposition lässt sich bei diesem Materialsystem keine Entstehung einer kumulativen Rauigkeit der aufgewachsenen Einzelschichten feststellen, die für eine lokale Erhöhung korrelierter Grenzflächenrauigkeiten mit steigender Anzahl an deponierten Schichten, wie es teilweise bei der Herstellung von gesputterten W/Si-Multilagen der Fall ist, sprechen würde [111]. In Abbildung 6.26 ist ein kompletter Querschnitt aus mehreren überlagerten TEM-Bildern dargestellt, bei dem auch die größte Si-Schicht sowie das 


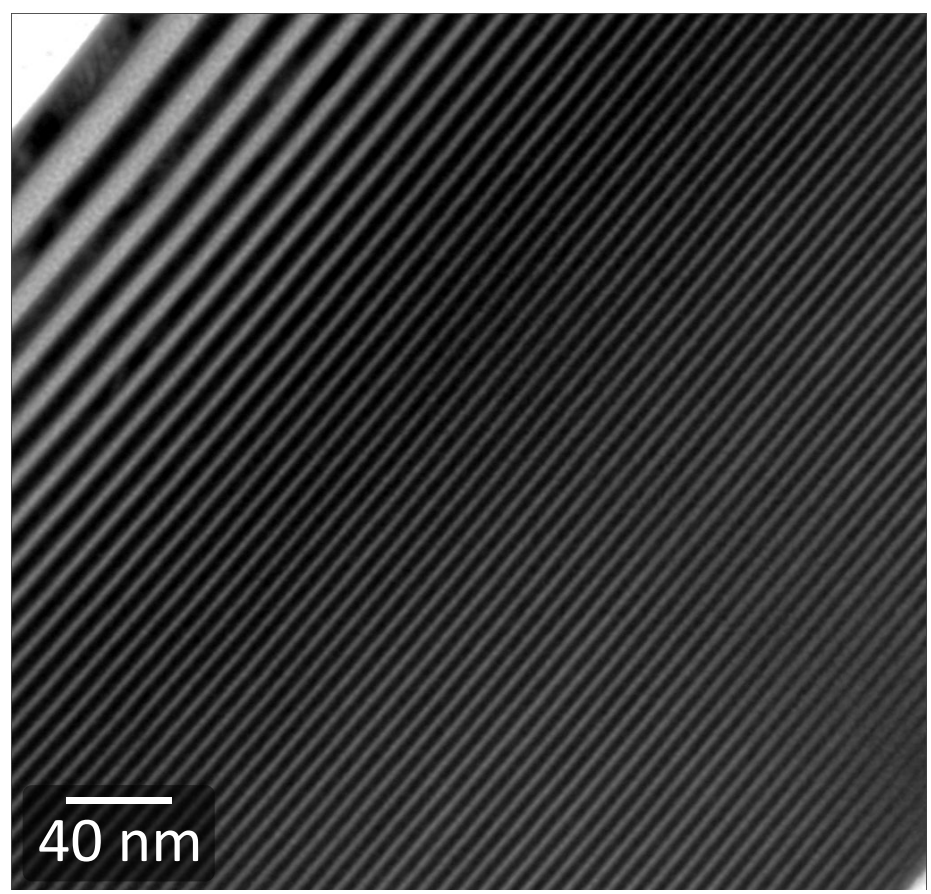

Abbildung 6.25: Querschnittsbild der MLL im TEM, bei der Si (hell) komplett amorph, W (dunkel) oberhalb einer Schichtdicke von $8 \mathrm{~nm}$ in einer kristallinen Struktur aufwächst. (Die Aufnahme wurde freundlicherweise von Matthias Hahn und Burkhard Roos angefertigt.)

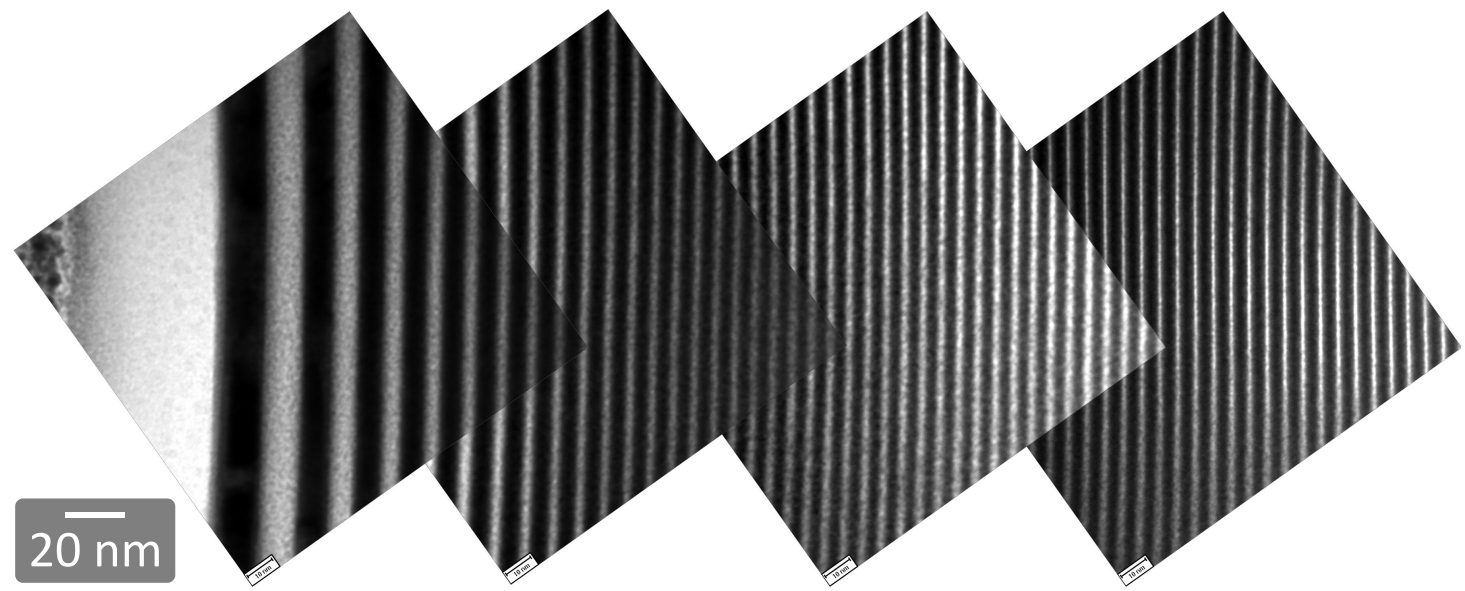

Abbildung 6.26: Überlagerte TEM-Querschnittsaufnahme der W/Si-MLL. Die gesamte Multischicht mit einem wurzelförmigen Anstieg der Einzelschichtdicken lässt keine Bildung einer kumulativen Rauigkeit erkennen. (Die Einzelaufnahmen der TEM-Bilder wurden freundlicherweise von Matthias Hahn und Burkhard Roos angefertigt.)

angrenzende im FIB deponierte Pt sichtbar ist. Die Gesamtschichtdicke wurde anhand dieses TEM-Bildes zu $418 \mathrm{~nm}$ gemessen.

Eine quantitative Schichtdickenanalyse an stark vergrößerten zusammengesetzten TEM-Aufnahmen ist in Abbildung 6.27 dargestellt. Dabei wurden nicht die einzelnen 


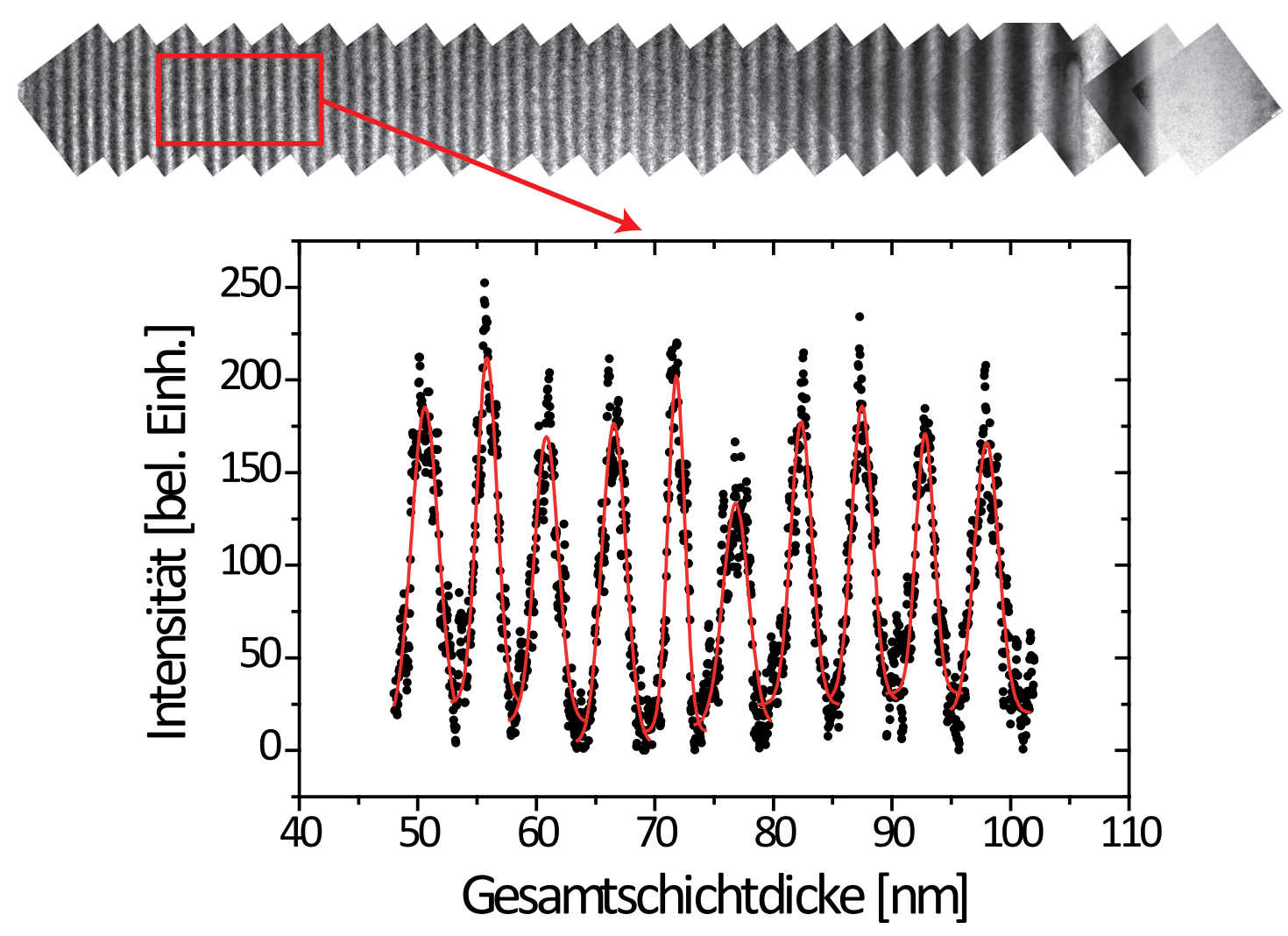

Abbildung 6.27: Schichtdickenauswertung der MLL mittels Positionsbestimmung der Einzelschichten aus stark vergrößerten TEM-Aufnahmen. Beispielhaft sind die Intensitätsprofile und zugehörige Gauß-Fits für Si-Schichten zwischen 50 und $100 \mathrm{~nm}$ Gesamtschichtdicke dargestellt. (Die Einzelaufnahmen der TEM-Bilder wurden freundlicherweise von Matthias Hahn und Burkhard Roos angefertigt.)

Schichtdicken, welche abhängig von den Kontrasteinstellungen der Bilder sind, direkt vermessen, sondern die mittleren Schichtpositionen mit Hilfe von Intensitätsprofilen (Änderungen der Grauwerte) bestimmt und anhand derer die zugehörigen Schichtdicken berechnet. Da bei diesem Verfahren nur die Doppelschichtdicken ermittelt werden können, ist es allerdings nur für annähernd periodische Strukturen geeignet. Die 10 dicksten Schichten, die sich nach dem Zonenplattenbildungsgesetz wesentlich in ihrer Dicke unterscheiden, wurden daher manuell ausgewertet. Anhand eines Ausschnitts des Schichtpakets wird demonstriert, wie die einzelnen Peaklagen der Si-Schichten per Gauß-Fits bestimmt wurden. Im Fall von W musste das kombinierte TEM-Bild lediglich invertiert und auf gleiche Weise ausgewertet werden.

Das zugehörige Ergebnis der Schichtdickenanalyse in Abbildung 6.28 vergleicht Sollund Ist-Schichtdicke der einzelnen Zonen. Bei den dünnsten Schichten stellte sich hierbei der größte Auslesefehler der TEM-Analyse heraus. Im Ergebnis wurde anstatt 
der angestrebten Dicken von 1,6 nm und größer für die ersten 15 Zonen eine mittlere Schichtdicke von 2,6 nm gemessen. Der Grund hierfür kann in einer ungenügenden Depositionsratenbestimmung genau in diesem Schichtdickenbereich gesehen werden. Im Bereich dickerer Schichten liegen die Daten oberhalb der Erwartung, was eventuell auf die manuelle Auswertungsmethode der Schichtdicken zurückgeführt werden könnte, zumal das direkte Ablesen der Einzelschichtdicken von der Kontrasteinstellung des jeweiligen Bildes beeinflusst wird. Die letzte und dickste Si-Schicht zeigt hierbei die größte Abweichung, welche als Folge einer $\mathrm{SiO}_{2}-$ Bildung an der Oberfläche entstanden sein könnte.

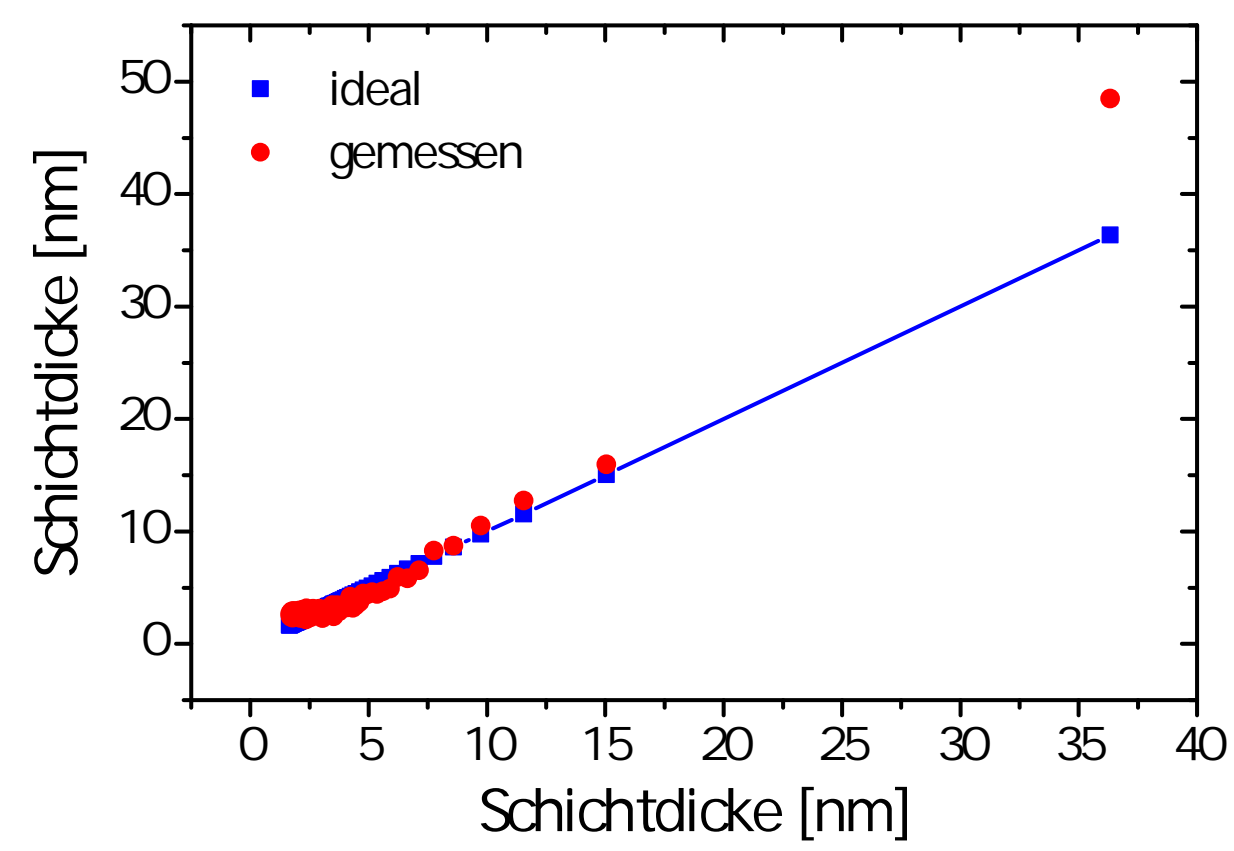

Abbildung 6.28: Mithilfe von TEM-Aufnahmen aus Abbildung 6.26 ermittelte Einzelschichtdicken der laserdeponierten MLL. Eine Abweichung zu den theoretischen Daten nach dem Zonenplattenbildungsgesetz zeigt sich hauptsächlich im Bereich dünner Schichten.

Zusammenfassend ist angesichts der letzten beiden Kapitel 6.2 und 6.3 deutlich geworden, wie umfangreich die Vorbereitungen auf die Herstellung eines solch komplexen Schichtsystems wie W/Si sein müssen, um hochpräzise Multischichten herstellen zu können, wie sie für die Fabrikation einer MLL benötigt werden. Trotz der auftretenden Effekte wie ein Schichtdickenverlust an den Grenzflächen und einer Durchmischung derselbigen durch Ionenimplantationen konnte mit der gepulsten Laserdeposition die Herstellung strukturell intakter, aperiodischer W/Si-Multischichten ermöglicht werden. 


\subsection{Eindimensionale MLL-Fabrikation mittels FIB}

Die Anfertigung der MLL im FIB begann mit der Deposition einer Pt-Schicht von etwa $10 \times 5 \times 5 \mu \mathrm{m}^{3}(\mathrm{~L} \times \mathrm{B} \times \mathrm{H})$ mittels des Elektronen- und Ionenstrahls auf dem hergestellten Schichtpaket an einer zuvor ausgewählten dropletfreien Oberflächenposition, um das Schichtsystem vor $\mathrm{Ga}^{+}-$Ionenbeschuss zu schützen und gleichermaßen eine ausreichende Stabilität der späteren Linse zu schaffen. Da sich die Gasinjektionsnadel, mit der das Pt in die Kammer geleitet wird, schräg zur Probenoberfläche befindet, kommt es am linken Bereich der Pt-Schicht zu einem Abschattungseffekt und somit zu einer leicht verringerten Schichtdicke (s. Abb. 6.29). Die Tatsache, dass die Multischicht auf dem $1 \mu \mathrm{m}$ dünnen Bereich eines $\mathrm{Si}_{3} \mathrm{~N}_{4}$-Substrats deponiert wurde, vereinfachte im nächsten Arbeitsschritt das Herauslösen des Schichtpakets mittels der Omniprobe, indem auf das aufwändige Freilegen durch sog. „regular cross sections“ (vgl. Kap. 5.1) verzichtet und direkt zum „U-cut“ übergegangen werden konnte. Dieser wurde, wie in Abbildung 6.29 rechts gezeigt, nicht unter $7^{\circ}$, sondern senkrecht zur Oberfläche getätigt, um die W/Si-Schichten einschließlich Substrat mit dem fokussierten Ionenstrahl zu durchschneiden.
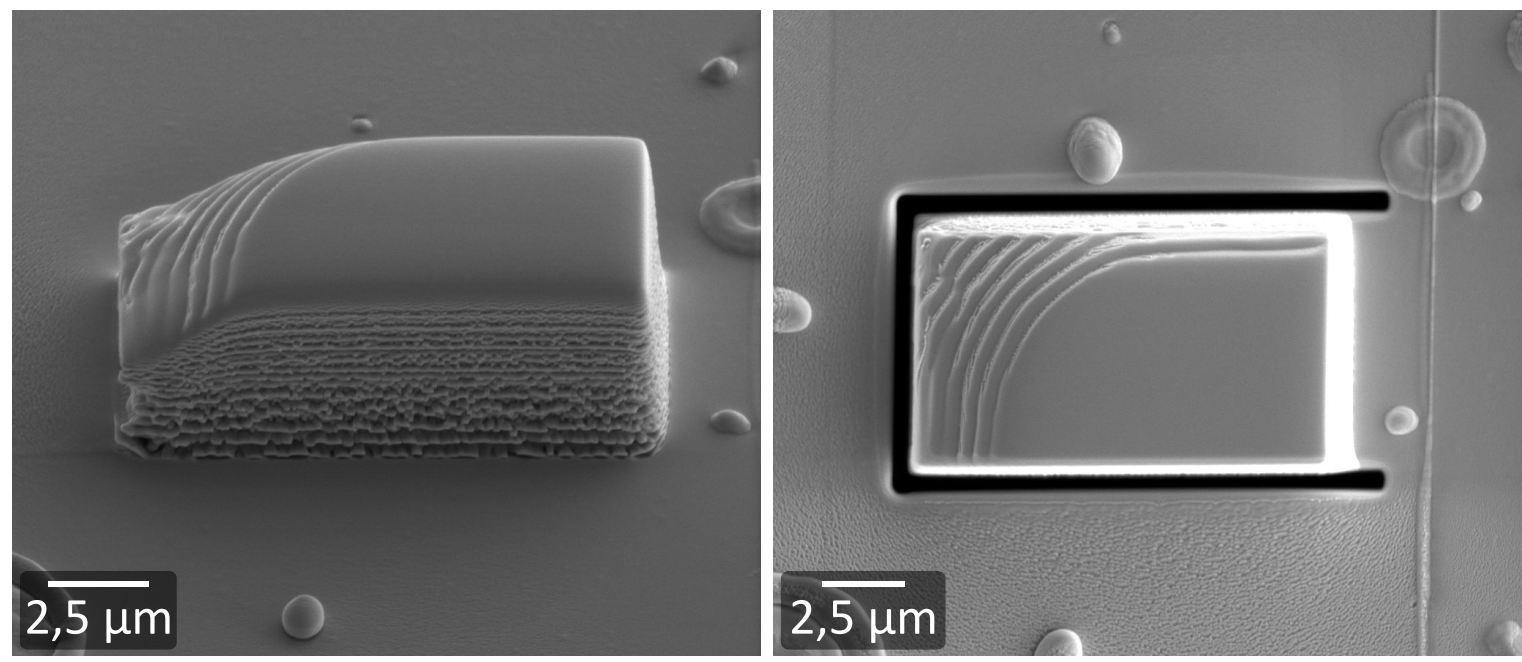

Abbildung 6.29: Erste Schritte der MLL-Herstellung mit Hilfe der fokussierten Ionenstrahltechnik.

Nach der Deposition einer $5 \mu \mathrm{m}$ hohen Pt-Schutzschicht kann der U-cut aufgrund der geringen $\mathrm{Si}_{3} \mathrm{~N}_{4}$-Substratdicke senkrecht zur Probenoberfläche durchgeführt werden.

Als Träger für die MLL wurde eine Omniprobe-Nadel aus W verwendet, deren konische Spitze zuvor mit dem Ionenstrahl auf einen Durchmesser von ca. $14 \mu \mathrm{m}$ begradigt wurde. Die Linse wurde hierbei so am unteren Ende der liegend auf dem Probentisch fixierten Omniprobe befestigt, dass nur die Pt-Schutzschicht mit 
der Stirnfläche der Spitze verbunden wurde, um das Schichtsystem selbst beim Annäherungsvorgang nicht zu beschädigen. Besonders anspruchsvoll ist dabei die Justage der richtigen Höhe von Linse und Omniprobe-Spitze, die in der REM-Aufnahme (s. Abb. 6.30 links) in die Bildebene hineinragt, sodass nur in der Kombination mit einer ionenerzeugten Darstellung, welche um $52^{\circ}$ versetzt abbildet, ein präzises Arbeiten möglich ist. Sobald der Kontakt der beiden Konstruktionselemente hergestellt wurde, konnte nach einer punktuellen Pt-Fixierung an der Verbindungsstelle die Omniprobe des Mikroskops abgetrennt und der Probentisch so gedreht werden, dass die Linse schließlich rechts und links der Auflagefläche mit Pt-Deposit verstärkt wurde (s. Abb. 6.30 rechts).
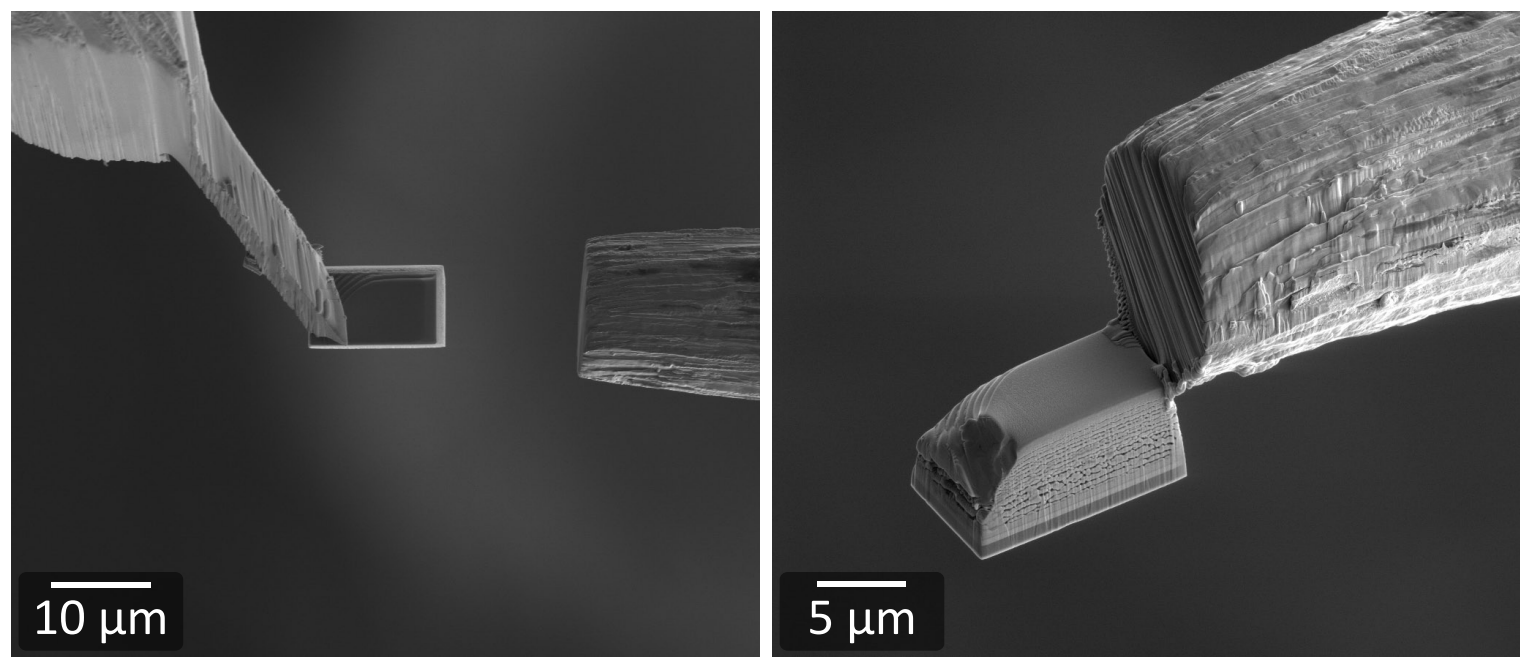

Abbildung 6.30: Annäherung und Fixierung der MLL an eine separate Omniprobe-Spitze. Damit die Schichtstruktur beim Annähern nicht beeinflusst wird, soll lediglich die Pt-Schutzschicht mit der Spitze in Berührung kommen.

Für einen optimalen Phasenschub musste der Multischicht-Rohling im Folgenden präzise ausgedünnt werden. Durch einen Offset-Winkel von $1,2^{\circ}$ zum einfallenden $\mathrm{Ga}^{+}$-Ionenstrahl wurde eine gleichmäßige Dünnung über die gesamte Höhe von ca. 6,5 $\mu \mathrm{m}$ gewährleistet (s. Abb. 6.31 oben links). Die Blendeneinstellungen beliefen sich standardmäßig auf $0,3 \mathrm{nA}$ und $0,1 \mathrm{nA}$ zum Vorschneiden auf eine optische Dicke der Linse von 3,26 $\mu \mathrm{m}$ sowie $30 \mathrm{pA}$ für eine letzte Politur der Schnittflächen. In der schrägen Ansicht der Linse in Abbildung 6.31 oben rechts lassen sich erstmals Substrat, Multischicht, elektronenstrahl-- und ionenstrahldeponiertes Pt sichtbar voneinander unterscheiden.

Im letzten Schritt galt es noch den vorderen Teil der Schichtstruktur so weit auszudünnen, dass er als abbildende Probe verwendet werden kann (s. Abb. 6.31 unten). 

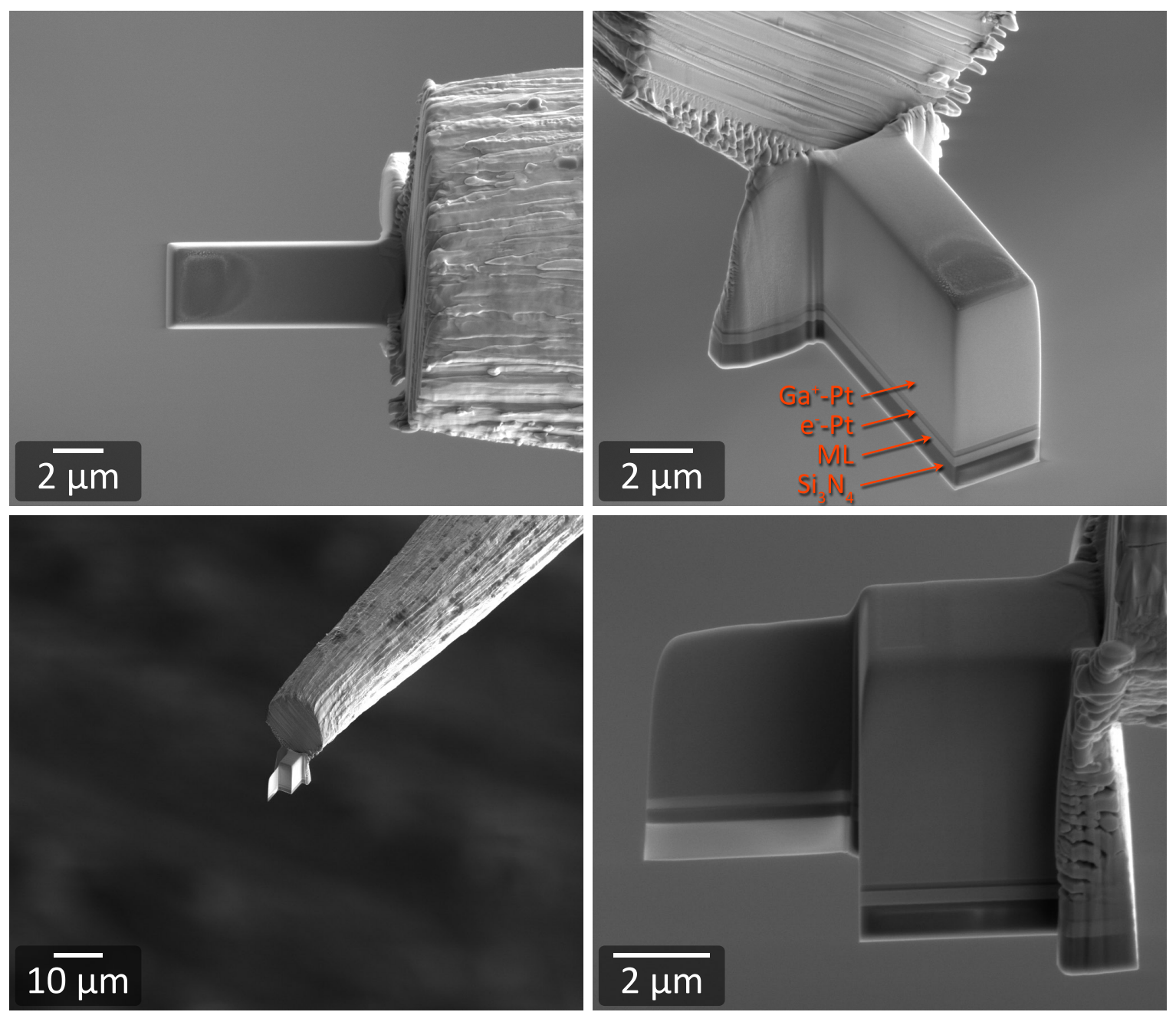

Abbildung 6.31: Oben: Erster Ausdünnungsprozess der phasenschiebenden MLL unter $1,2^{\circ}$ OffsetWinkel zum $\mathrm{Ga}^{+}-$Ionenstrahl bis zur gewünschten optischen Tiefe von $3,26 \mu \mathrm{m}$. Nach feinem Ionenstrahlpolieren sind die Grenzen der einzelnen Schichtsegmente erstmals sichtbar. Unten: Weiteres Ausdünnen des vorderen Teils der MLL. Mit einer optischen Tiefe kleiner als $200 \mathrm{~nm}$ ist dieser Bereich des Multischichtpakets zur Abbildung mit separaten Fokussierungsoptiken gedacht.

Durch präzises Ionenstrahlpolieren wurde diesbezüglich entsprechend viel Material von einer Seite der Linse abgetragen, um eine für die Abbildung mit Röntgenstrahlung adäquate optische Tiefe zu erreichen. Nach dem letzten Ausdünnungsvorgang ist dieser Teil des Multischichtpakets nur noch $170 \mathrm{~nm}$ dick und in der Seitenansicht in Abbildung 6.31 unten rechts für den Elektronenstrahl bereits teilweise durchsichtig.

Die fertiggestellte Kombination aus linienfokussierender Linse und Probe ist in Abbildung $6.32 \mathrm{zu}$ sehen, bei der die jeweiligen Strahlengänge der transmittierenden Röntgenstrahlung im geplanten Synchrotron-Experiment skizziert sind. Nach der Arbeit am Mikroskop wurde die insgesamt mehrere Zentimeter lange Omniprobe- 


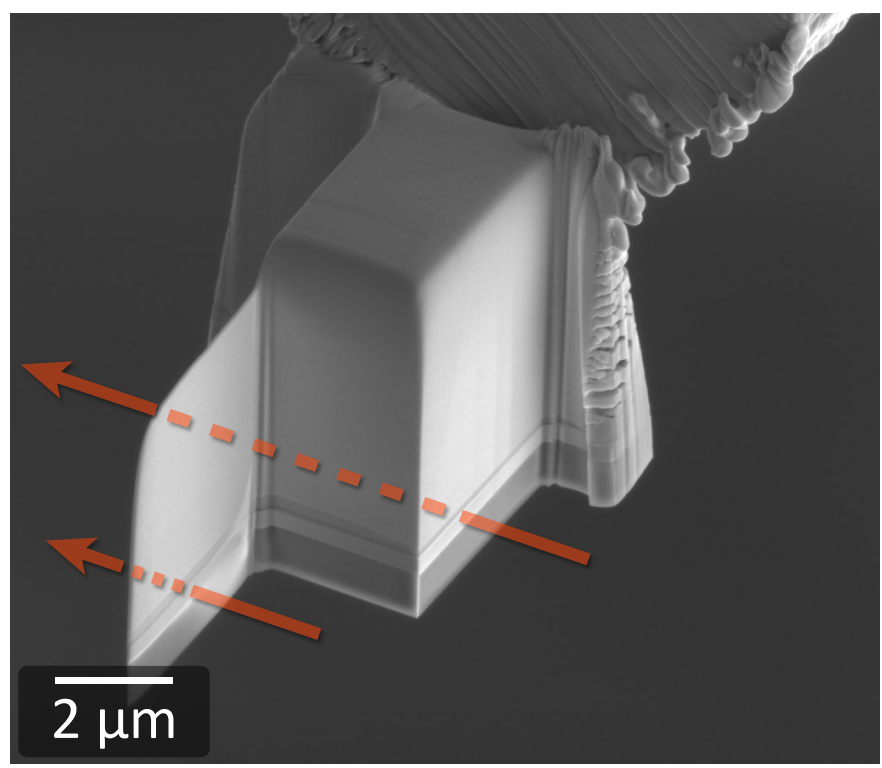

Abbildung 6.32: Aufteilung der fertig präparierten MLL in zwei verschiedene Schichtsegmente für mögliche Synchrotron-Experimente: Der dicke Bereich soll einfallende Röntgenstrahlung linienfokussieren, der dünnere mit Röntgenstrahlung als Probe vergrößert abgebildet werden [185].

Spitze, die einen Endradius von einigen Millimetern aufweist, in einen speziellen Halter eingeklebt. Für darauf folgende Messungen ist sie somit einfach zu handhaben.

\subsection{Strahlcharakteristik am Synchrotron}

Zur Vorbereitung auf die Messung der Fokuseigenschaften der eindimensionalen MLL, welche von Mitarbeitern der AG Salditt (Institut für Röntgenphysik, Universität Göttingen) am Beschleunigerring PETRA III (DESY, Hamburg) durchgeführt wurde, musste diese zunächst in mehreren Schritten im Strahlengang der KB-Optik ausgerichtet werden. Zum Zeitpunkt der Experimente betrug die Strahlgröße im KB-Fokus $320 \times 200 \mathrm{~nm}(\mathrm{FWHM})$, sodass mit der hergestellten $400 \mathrm{~nm}$ dicken MLL wie geplant eine off-axis-Struktur erzeugt wurde. Zu Beginn der Messung wurde ein Wellenleiter zwischen Quelle und Linse in den Strahlengang eingebracht und nach einer Vorjustage ein Hologramm der MLL erstellt (s. Abb. 6.33), welches der REM-Aufnahme der MLL im FIB ähnelt (vgl. Abb. 6.31 unten rechts). Da der Detektor aus vier Quadranten zusammengesetzt ist, ergeben sich eine horizontale und vertikale Linie, innerhalb derer keine Bildinformation vorliegt. Im rechten oberen Teil ist das Ende der W-Spitze zu erkennen, an die die MLL angebracht wurde. In der Mitte ist der dickere Teil der Linse 


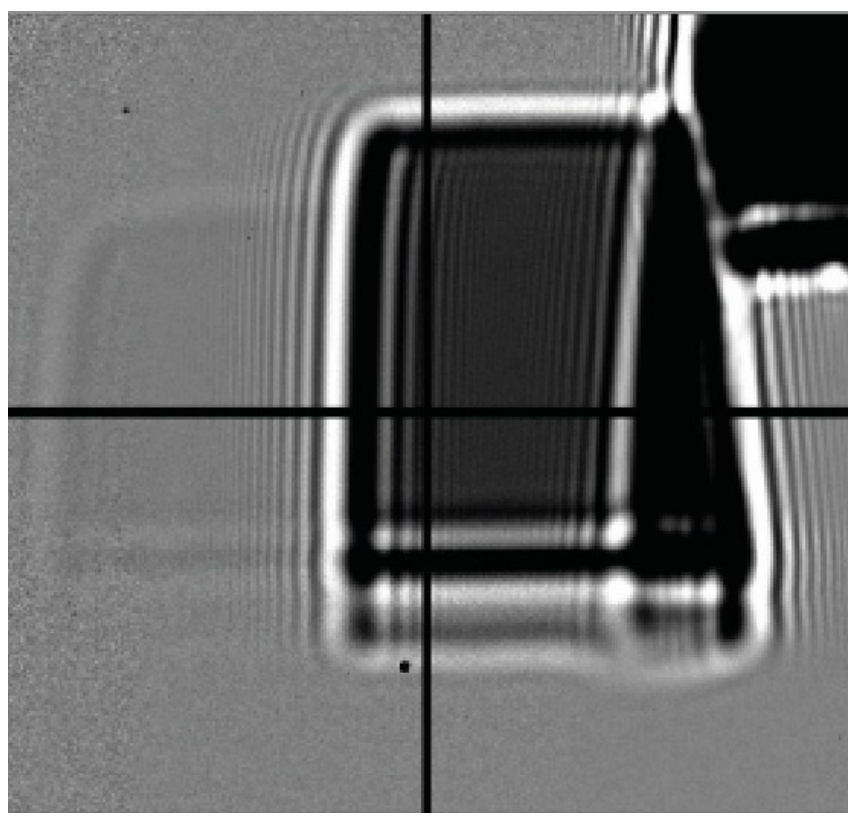

Abbildung 6.33: Holographie-Bild der MLL, aufgenommen während der Vorjustage mit einem Wellenleiter im KB-Strahlengang [185]. Von links nach rechts betrachtet ist zuerst der dünnere, als Probe fungierende Teil der MLL, dann der phasenschiebende Abschnitt und schließlich das Ende der Omniprobe-Spitze erkennbar. Einen optischen Vergleich liefert die REM-Aufnahme der MLL in Abbildung 6.31 unten rechts.

dargestellt, während in den beiden linken Quadranten sogar der auf $170 \mathrm{~nm}$ ausgedünnte Abschnitt schwach zu erkennen ist.

Zur Feinjustage wurde die Linse direkt im KB-Strahlengang (ohne Wellenleiter) mit einer nahe der Linse befindlichen Photodiode für verschiedene Verkippungswinkel entlang der Schichtstruktur abgescannt, um sie anhand maximaler Kantenschärfe (z. B. beim Übergang von Multischicht zu Pt-Deposit) genau senkrecht zum KB-Strahl zu justieren. Um eine lokale und somit insgesamte Effizienzerhöhung zu erreichen, wurde die Linse im Anschluss zur Erfüllung der Bragg-Bedingung für äußere Zonen um 22 mrad verkippt. Dieser Wert stellt den optimalen Winkel für eine Schichtperiode von ca. $2 \mathrm{~nm}$ dar. Nach der Wahl der Justageparameter wurden mit Hilfe des PILATUSDetektors bei verschiedenen y-Positionen mehrere Beugungsbilder im Fernfeld mit einer jeweiligen Belichtungszeit von 0,1s aufgenommen, welche in Abbildung 6.34 oben als zusammengesetzte Intensitätsverteilung $I\left(q_{x}, q_{y}\right)$ aufgeführt sind. Dabei wurde die Mitte des Bildes durch einen beam stop ausgeblendet, um den Detektor nicht zu überstrahlen, sodass dort keine Photonen registriert wurden. Ferner zeigen sich vertikale Ausläufer des KB-Nullstrahls. Das auffallend stark verbreiterte Beugungsbild im $q$-Raum deutet bereits auf sehr kleine Intensitätsvariationen im Fokus hin, was 

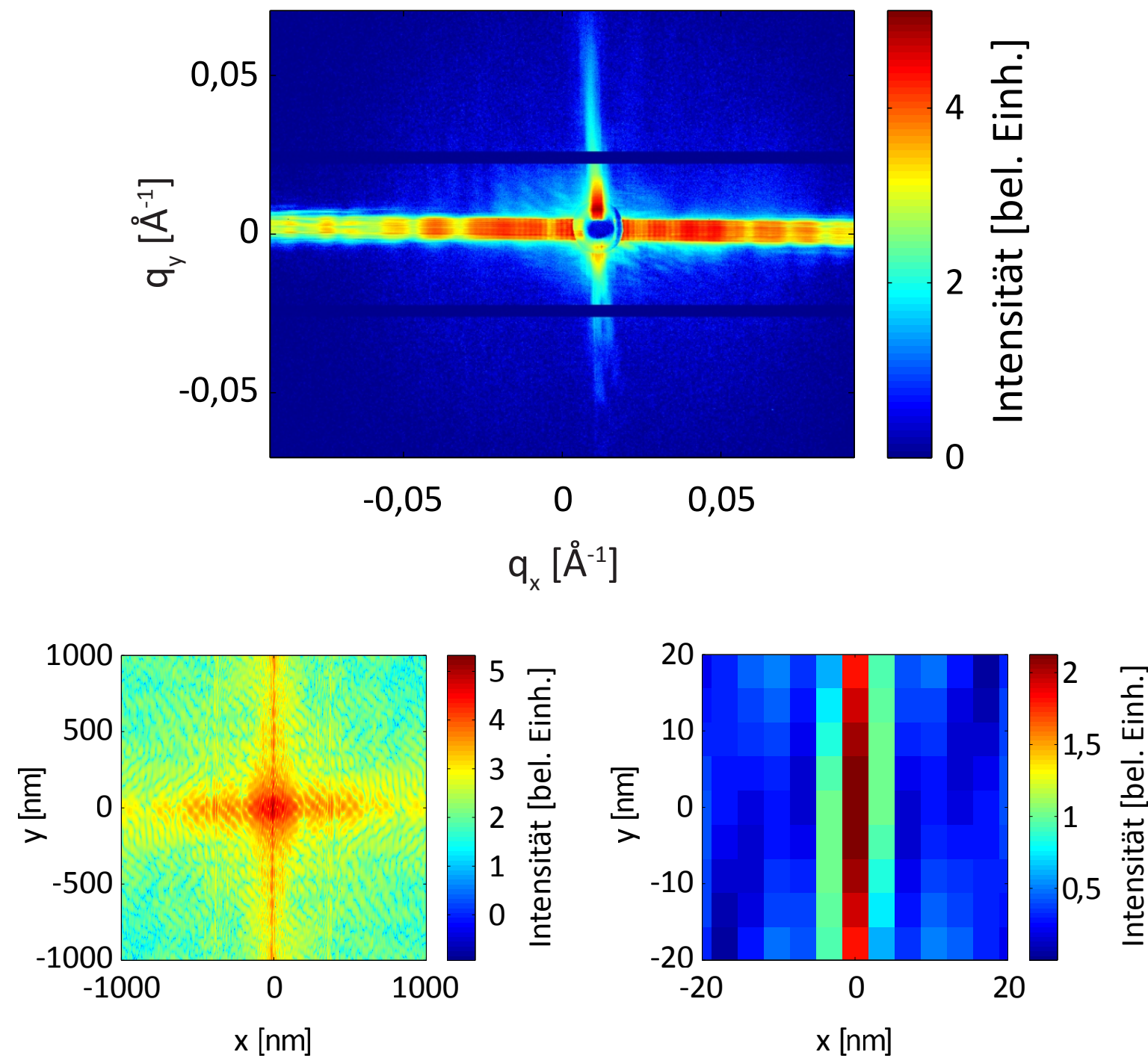

Abbildung 6.34: Zusammengesetzte Intensitätsverteilung der MLL im Fernfeld (oben) und zugehörige 1D-Autokorrelation des vertikal integrierten Profils in logarithmischer (unten links) und linearer Darstellung (unten rechts). Das stark verbreiterte Beugungsbild deutet auf sehr kleine beugende Strukturen im Fokus hin, die nach der Anfertigung einer Autokorrelation eine sehr schmale Intensitätsverteilung im Maximum zeigen. Ein zugehöriger Linienscan ist in Abbildung 6.36 dargestellt. (Die Daten wurden freundlicherweise von Sven Philip Krüger zur Verfügung gestellt [185].)

auf eine sehr scharfe Fokussierung schließen lässt. In diesem Fall ist die aufgeweitete Intensität sogar seitlich durch das Strahlrohr begrenzt und nicht vollständig erfasst worden, sodass mit einem weiteren vor dem Strahlrohr befindlichen Detektor deren halber Öffnungswinkel zu 17,3 mrad bestimmt wurde.

Zur Auswertung des zusammengesetzten Beugungsbildes wurde von Sven Philip Krüger am Institut für Röntgenphysik (Universität Göttingen) eine eindimensionale Auto- 

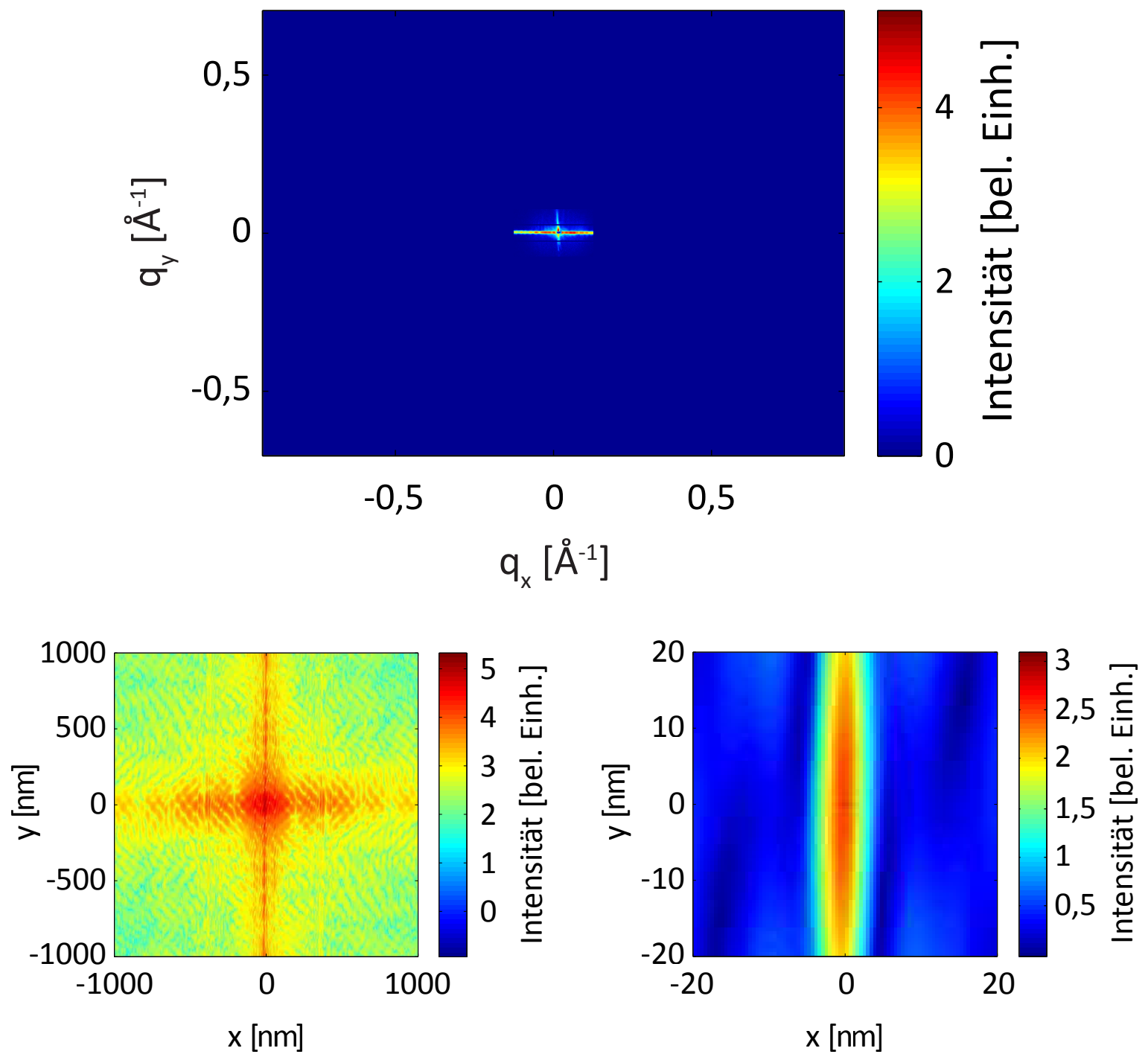

Abbildung 6.35: Auswertung des Strahlprofils mittels eines „regridings“ der Fernfeldbeugung (oben), um eine höhere Aufösung der Autokorrelation zu erhalten (unten). Ein zugehöriger Linienscan ist in Abbildung 6.36 dargestellt. (Die Daten wurden freundlicherweise von Sven Philip Krüger zur Verfügung gestellt [185].)

korrelationsfunktion $g(x) \propto F F T^{-1}\left[I\left(q_{x}\right)\right]$ des vertikal integrierten Intensitätsprofils angefertigt, welche in Abbildung 6.34 unten in logarithmischer (links) und linearer Darstellung (rechts) aufgezeigt ist. Bei Betrachtung des linear dargestellten Graphen ist die schmale Intensitätsverteilung im Maximum besonders gut zu entnehmen. Ein Linienscan der Autokorrelation, welcher in Abbildung 6.36 durch rechteckige Datenpunkte gekennzeichnet ist, offenbart eine enorm kleine Halbwertsbreite unter $7 \mathrm{~nm}$. Die dabei ermittelte Strahlintensität liegt mit $10^{9} \mathrm{ph} / \mathrm{sec}$ ein bis zwei Größenordnungen 


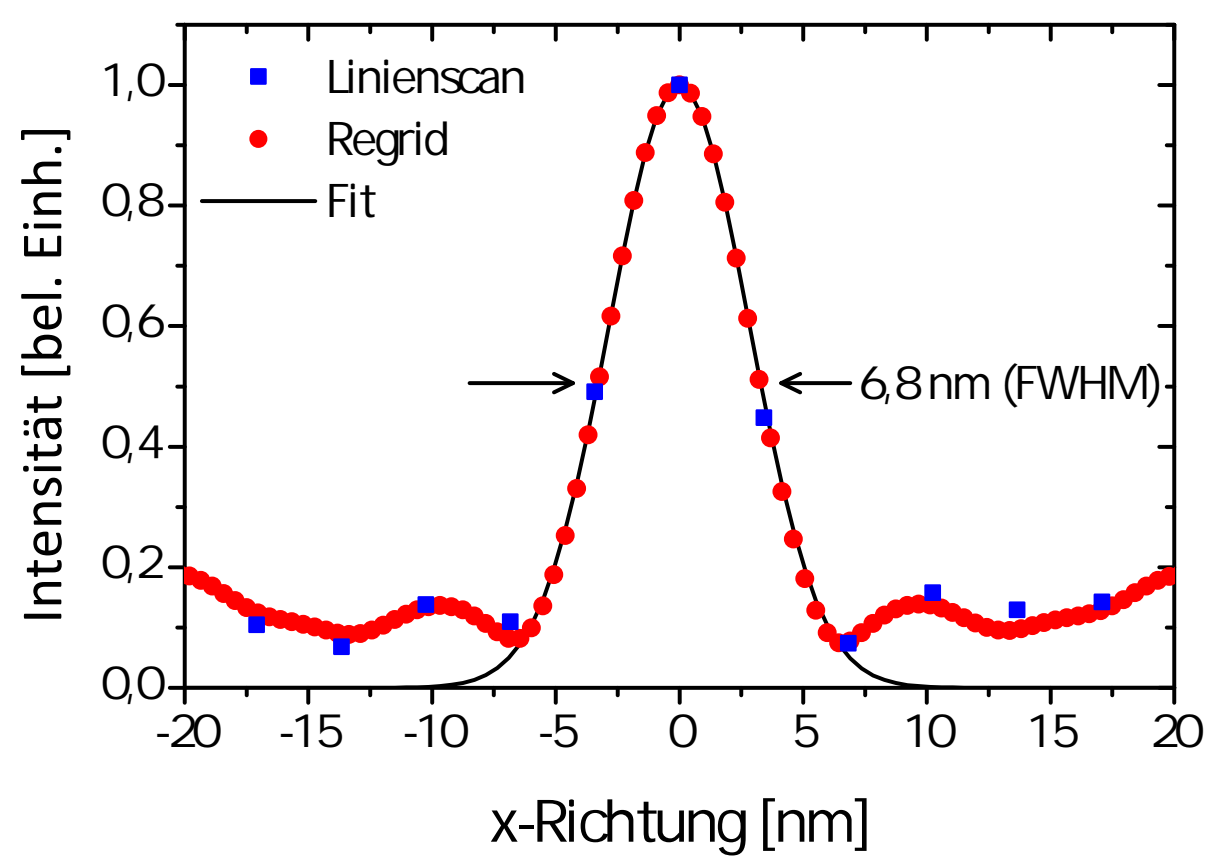

Abbildung 6.36: Bestimmung der Intensitätsverteilung im Fokus der MLL-Optik anhand von durchgeführten Fernfeldmessungen, welche mittels der Anfertigung zweier Autokorrelationen (Original und „Regrid“) eine Fokusgröße kleiner als $7 \mathrm{~nm}$ (FWHM) ergeben. (Die Daten wurden freundlicherweise von Sven Philip Krüger zur Verfügung gestellt [185].)

über dem Photonenfluss, der normalerweise zur kohärenten Strahlerzeugung mittels eines Wellenleiters gemessen wurde [204].

Eine verbesserte Auflösung des Linienscans lässt sich mit der sog. „regrid-Methode“ erreichen, bei der das originale Fernfeld-Beugungsmuster in ein größeres Gitter konstanter Intensität eingebettet wird (s. Abb. 6.35 oben), um eine ausreichend abgetastete Autokorrelation zu erhalten (unten). Das Ergebnis in Abbildung 6.36 zeigt mit dieser Methode schließlich einen Gauß-Fit des Linienscans mit einer extrem kleinen Halbwertsbreite von nur $6,8 \mathrm{~nm}$.

Trotz des großartigen Ergebnisses übersteigt der Wert den theoretisch berechneten im Design-Kapitel 6.1, was hier kurz diskutiert werden soll. Einer der Gründe für die Abweichung der gemessenen Halbwertsbreite ist mit Sicherheit in der experimentell erreichten minimalen Schichtdicke der MLL zu sehen. Da diese mit einer ungefähren Dicke von $\Delta r=2,6 \mathrm{~nm}$ anstatt der geplanten 1,6 nm hergestellt wurde ergibt sich die Abschätzung der Fokusgröße zu $R_{\text {ber. }}=2 \cdot 1,22 \Delta r=6,3 \mathrm{~nm}$, welche mit den aus der Autokorrelation erhaltenen Werten sehr gut übereinstimmt. Weiterhin konnte während des Experiments nicht genau beobachtet werden, welcher Teil der Linse bzw. welche Zonen aktiv beleuchtet wurden, zumal die Strahlgröße im Experiment kleiner als die 

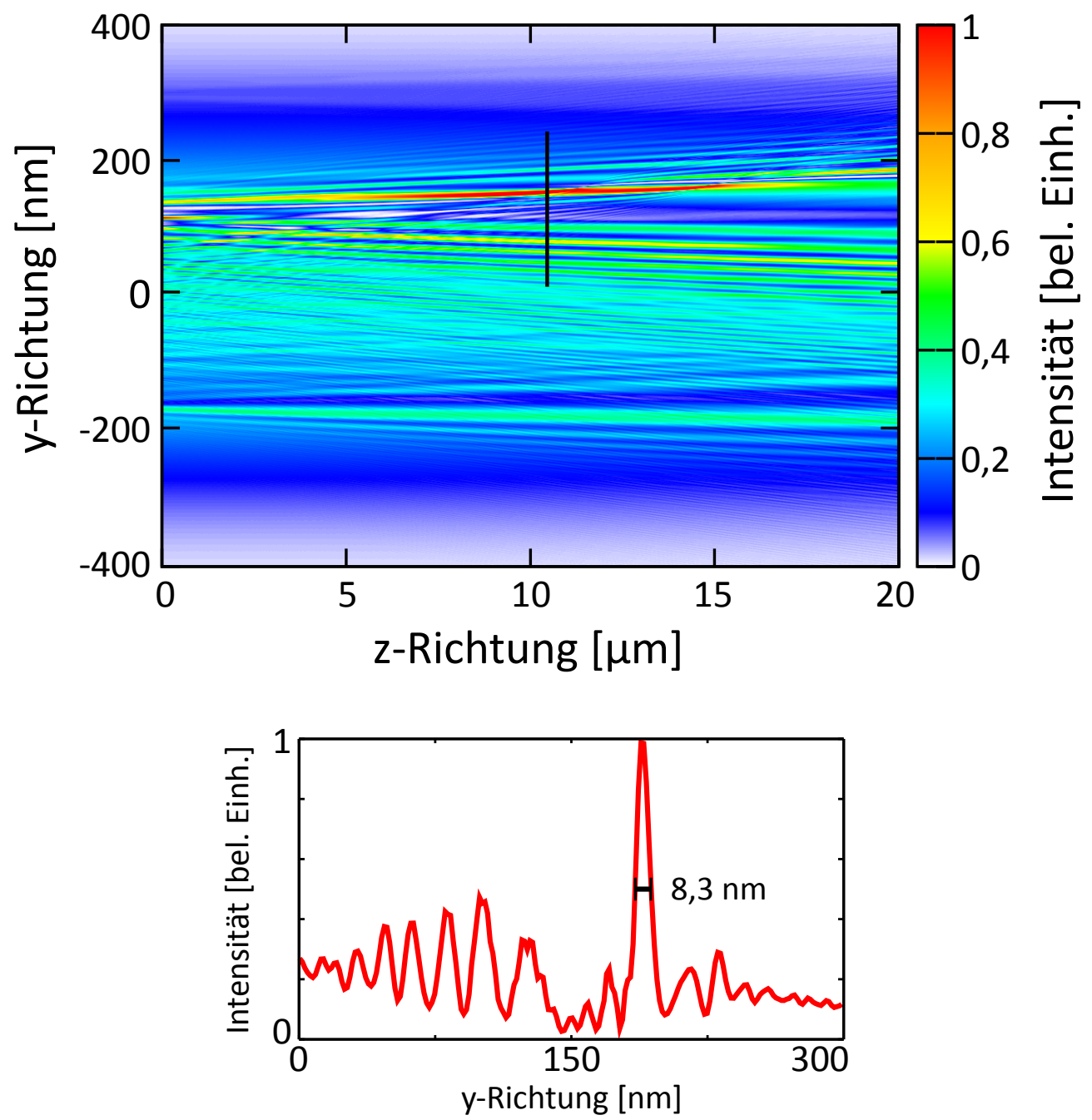

Abbildung 6.37: Simulierte Strahlpropagation der phasenschiebenden MLL unter Berücksichtigung von Volumeneffekten und dem im Experiment angenommenen Offsetwinkel von $22 \mathrm{mrad}$. Im Vergleich zur Simulation der ideal dünnen MLL (vgl. Abb. 6.3) scheinen dünnere Zonen im stark gestörten Wellenfeld nicht zu einer Fokussierung beizutragen, sodass der Linienscan (unten) eine theoretische Aufösung von 8,3 nm zeigt. (Die Simulationsdaten wurden freundlicherweise von Aike Ruhlandt zur Verfügung gestellt [185,187].)

gesamte MLL war. Daher wäre es möglich, dass die dünnsten Zonen nicht zu der gemessenen Fokussierung beigetragen haben.

Selbst bei einer vollen Beleuchtung der MLL ist anhand der Ergebnisse einer nachträglichen von Aike Ruhlandt am Institut für Röntgenphysik (Universität Göttingen) angefertigten Simulation der Strahlpropagation nicht gesichert, ob mit den dünnen Zonen effizient fokussiert wurde [187]. Als Ursache hierfür wäre eine nicht optimale Wahl der optischen Tiefe von 3,26 $\mu \mathrm{m}$ der MLL-Struktur zu nennen, wie sie erst- 
mals durch Berücksichtigung von Volumendiffraktionseffekten in die Simulation in Abbildung 6.37 miteinbezogen wurde. Dazu wurde anhand einer sog. „multisliceMethode" die Multischichtstruktur in eine große Anzahl paralleler Schnitte zerlegt und die Fresnel-Propagation von einer zur nächsten Ebene betrachtet. Im Ergebnis zeigt sich hinter der MLL ein gestörtes Wellenfeld, welches neben einer größeren Schärfentiefe und höherem Untergrundrauschen eine Verbreiterung des Fokus von $R_{\text {sim. }}=3,8 \mathrm{~nm}$ (vgl. Abb. 6.3) auf 8,3 nm aufweist. Dabei hat es den Anschein, als würden dünnere Zonen im Bereich von -180 bis $-200 \mathrm{~nm}$ mit Aspektverhältnissen teilweise größer als 1000 : 1 nicht zu einer Fokussierung beitragen, was entweder für eine weitere Optimierung der Simulation oder für einen zu großen Beugungswinkel spricht. Der propagierende Strahl würde aufgrund der im Vergleich zur Fokuslänge großen optischen Tiefe mit weiter innen gelegenen Zonen interferieren. Bei der objektiven Einschätzung dieser Simulation muss außerdem noch berücksichtigt werden, dass in den Berechnungen der Strahlpropagation keine Grenzflächenrauigkeiten innerhalb der Zonen erfasst wurden.

Insgesamt hat diese Messung das hohe Potential eines kombinierten Systems aus KBund laserdeponierter MLL-Optik gezeigt, um harte Röntgenstrahlung im sub-10 nmBereich zu fokussieren. Die durchgeführten Fernfeld-Messungen sprechen allesamt für eine erreichte Fokusgröße kleiner als $7 \mathrm{~nm}$, welche während einer zukünftig geplanten Strahlzeit durch bildgebende Experimente direkt im Fokus bestätigt werden soll. Diese außerordentlichen Ergebnisse werden als durchaus zukunftsträchtig gesehen und könnten mit dem mittels reflektiven Optiken erreichten, derzeit bestehenden Rekord von $7 \mathrm{~nm}$ Auflösung in Wettbewerb treten [184]. Die momentan beste Auflösung einer MLL für harte Röntgenstrahlung liegt bei $13 \mathrm{~nm}$ Linienfokusgröße [75] und konnte hier deutlich unterschritten werden. Darüber hinaus ist die hohe gemessene Effizienz der fokussierten kohärenten Röntgenstrahlung für viele röntgenmikroskopische Anwendungen wie die Phasenkontrast-Tomographie aufgrund kurzer Belichtungszeiten von besonderem Vorteil. 


\subsection{Design und Herstellung zweidimensionaler Multilagen-Laue-Linsen}

Da eine eindimensionale MLL im Vergleich zu einer konventionellen Zonenplatte aufgrund ihrer planaren Zonenstruktur allein zur Erzeugung eines Linienfokus dient, können zu untersuchende Proben nur in der fokussierten Ebene des Röntgenstrahls abgescannt werden. Dennoch besteht auch hier die Möglichkeit, einen realen Punktfokus zu generieren, ohne auf die essentiellen Vorteile einer multilagenbasierten Laue-Linse (kleinste Zonenbreiten und hohe Aspektverhältnisse) verzichten zu müssen. In der Literatur gibt es bereits erste gelungene Versuche, zwei unabhängige MLL in gekreuzter Geometrie im Synchrotron-Strahlengang auszurichten [102,126]. MASER et al. konnten auf diese Weise bei einer Energie von $12 \mathrm{keV}$ einen zweidimensionalen Spot von $25 \times 27 \mathrm{~nm}^{2}$ erzielen, wobei hochstabile Justageeinheiten vonnöten waren, um die auf die beiden Einzellinsen wirkende, auflösungsmindernde Vibrationen einzuschränken. Im folgenden Abschnitt wird nun ein alternativer Demonstrator einer zweidimensionalen MLL präsentiert, welcher einfallende Röntgenstrahlung durch ein festes und somit in sich vibrationsfreies Verbundsystem von senkrechter und waagrechter Einzellinse in einem Punkt konzentrieren soll.

Die Designparameter sowie die Systemauswahl für eine in dieser Arbeit hergestellten 2D-MLL wurden wiederum für harte Synchrotronstrahlung gewählt, für welche im dazu konzipierten Experiment ebenfalls eine Vorfokussierung durch ein KB-Spiegelsystem vorgesehen war (vgl. Abb. 6.1). Durch den Einsatz eines im FIB fabrizierten Linsensystems sollte sodann eine weitere Punktfokussierung unternommen werden. Abbildung 6.38 verdeutlicht hierzu die gekreuzte Anordnung von senkrechter und waagrechter Einzellinse, welche zur Generierung des zweidimensionalen Fokus führt. Die experimentellen Anforderungen entsprachen demzufolge jenen für den eindimensionalen Fall: Sämtliche Herstellungsparameter wie Wellenlänge, Fokuslänge, Anzahl der hergestellten Zonen, Einzel- und Gesamtschichtdicke sowie optische Tiefe wurden aus Kapitel 6.1 übernommen. Aufgrund der Ergebnisse im eindimensionalen Fall (vgl. Kap. 6.5) kann auch hier von einer finalen Fokusgröße von ungefähr $7 \mathrm{~nm}$ ausgegangen werden. Der Einfachheit halber sollen alle dazu benötigten Schichtsegmente aus einem einzigen Substrat herausgeschnitten werden, weshalb die waagrechte und senkrechte Brennweite $f_{1}$ und $f_{2}$ auf der optischen Achse genau um $\Delta t$ verschoben sein werden. Die Form eines idealen Kreisfokus wird daher eher der einer Ellipse gleichen. 


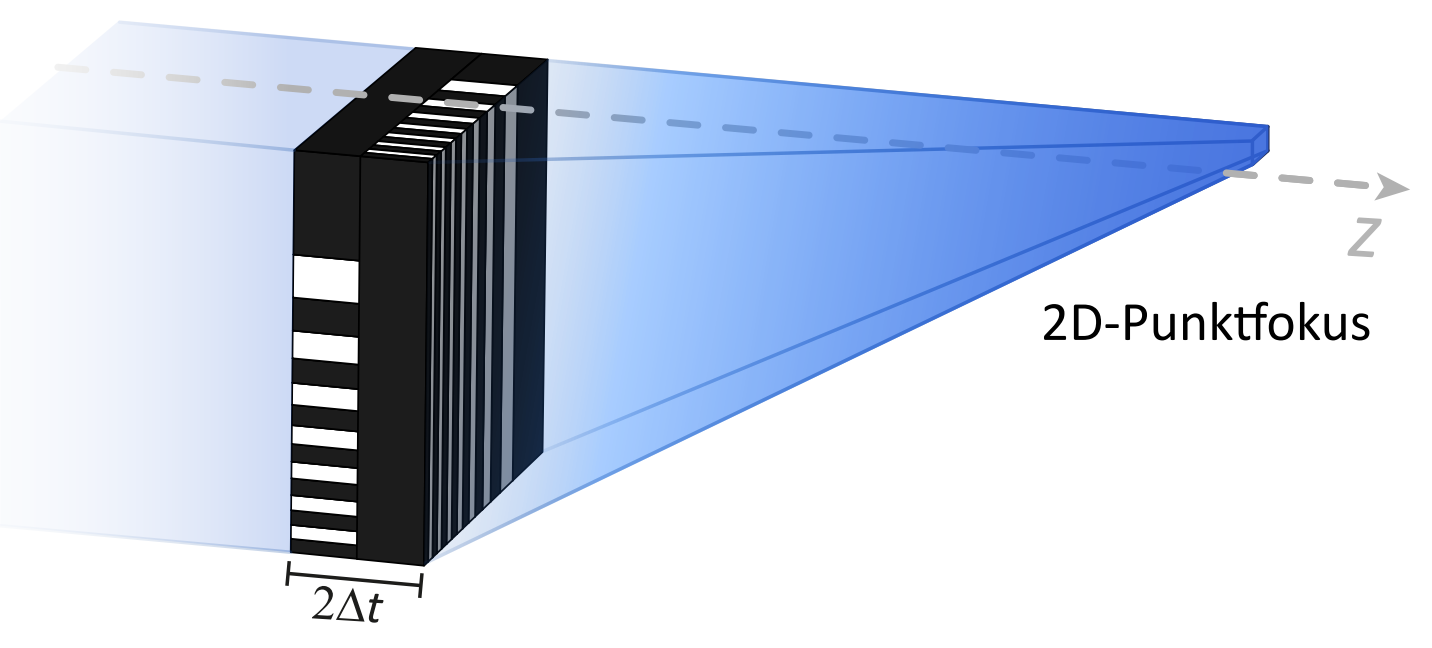

Abbildung 6.38: Schematische Darstellung einer gekreuzten MLL, mittels derer aus Kombination zweier um $90^{\circ}$ versetzte Einzellinsen ein 2D-Punktfokus generiert werden kann.

Die Herstellung des punktfokussierenden Linsensystems im FIB wurde maßgeblich durch die Hilfe von Volker Radisch unterstützt und vollzog sich zunächst in gleicher Weise wie die der eindimensionalen MLL. In Abbildung 6.39 ist dazu das mittels U-cut vorgeschnittene Schichtpaket gezeigt (links), welches im nächsten Schritt herausgelöst werden soll. Im unteren Teil des REM-Bildes ist jene Substratstelle ersichtlich, an der die eindimensionale Laue-Linse entnommen wurde.

Der erste Querschnitt der 2D-MLL wurde schließlich aus dem Substrat herausgelöst, an einer separaten Omniprobe-Spitze angebracht und auf die für einen Phasenschub geforderte optische Tiefe gedünnt. Da dieser in senkrechter Ausrichtung mit der zweiten Einzellinse verknüpft werden sollte, musste die liegende Omniprobe-Spitze im nächsten Prozessschritt um $90^{\circ}$ gedreht in stehender Orientierung auf dem Probentisch befestigt werden (s. Abb. 6.40 links) um die beiden Teilsegmente rechtwinklig miteinander vereinen zu können. Auf der Substratseite musste mit dem Ausdünnvorgang der zweiten Einzellinse bereits vor der Fixierung an das erste Schichtsegment begonnen werden. Bei einer vorschnellen Kontaktierung mit anschließendem Ausdünnen wäre sonst neben einer nicht zu verhindernden Redeposition von abgetragenem Schichtmaterial auf die erste MLL die Verbindungsfläche der zweiten zum Ionenpolieren nicht vollständig zugänglich. Erfreulicherweise konnte dieser Prozess aufgrund der geringen Substratdicke bereits vor dem endgültigen Herausheben des Schichtpakets aus dem Substrat erfolgen. Abbildung 6.40 rechts zeigt dazu in einer Aufsicht das fertig gedünnte Schichtsegment, welches aus Gründen der Stabilität nicht vollständig mit dem U-cut vorgeschnitten wurde, sondern zwei Verbindungen zum Substrat erhalten worden sind. 


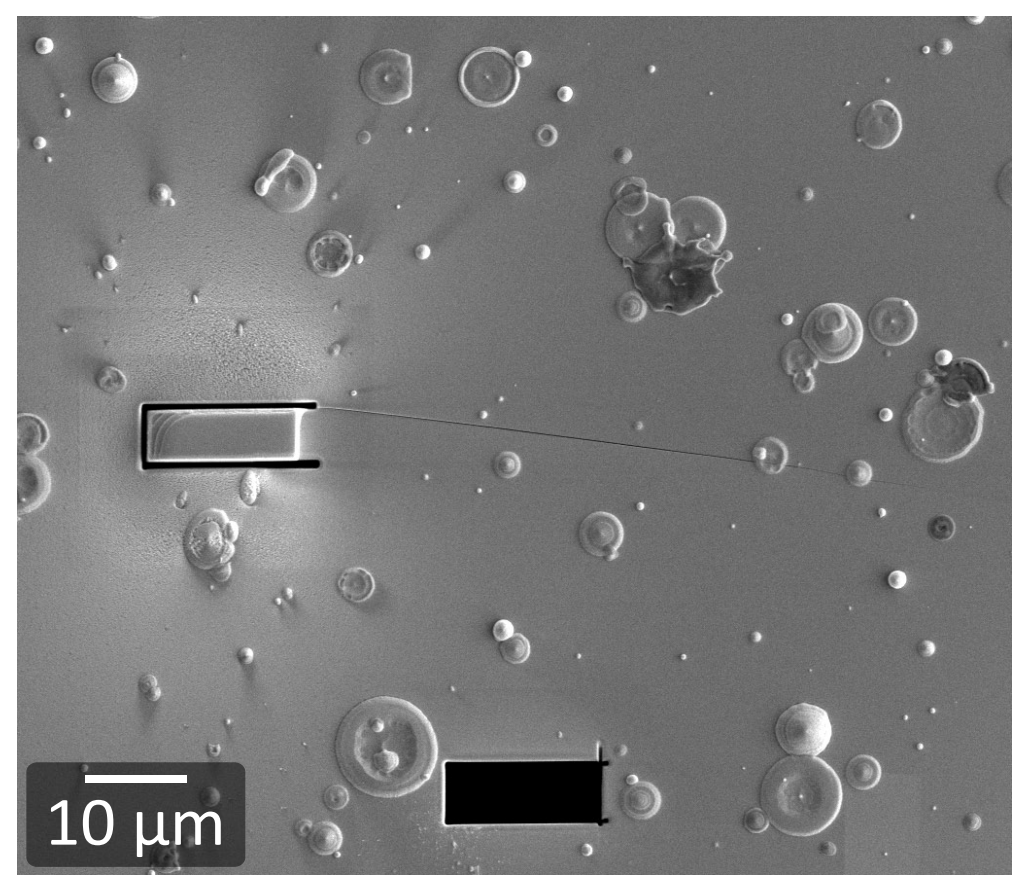

Abbildung 6.39: REM-Aufnahme der W/Si-Schichtoberfläche im FIB. Im unteren Teil ist jene Substratposition erkennbar, an der die eindimensionale MLL herausgehoben wurde. Links wurde hingegen mit dem Vorschneiden eines weiteren Querschnitts begonnen.
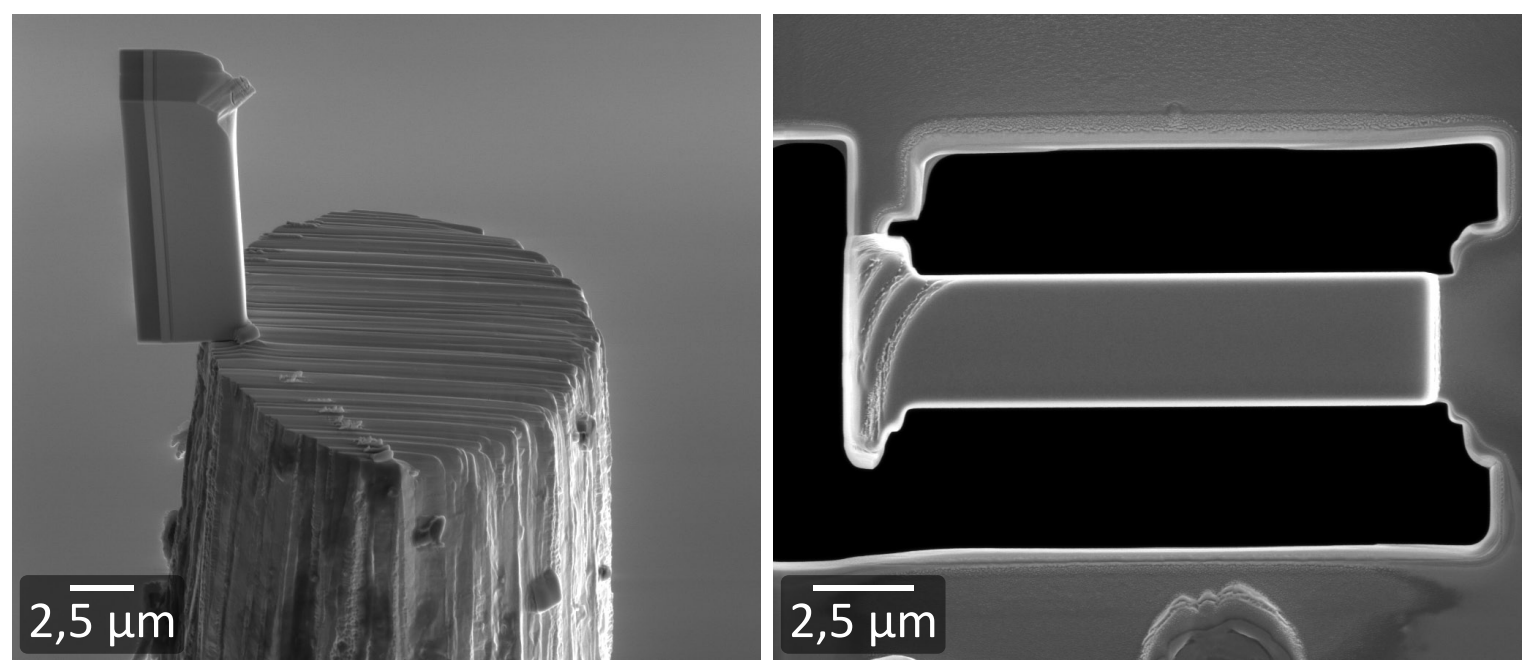

Abbildung 6.40: Senkrechte Ausrichtung der ersten Teillinse samt Spitze (links) als Vorbereitung zur Annäherung des zweiten Schichtpakets, bei dem das Ausdünnen direkt im Substrat erfolgen musste (rechts in Aufsicht), damit die Seitenflächen der beiden Einzellinsen exakt miteinander kontaktiert werden konnten.

Nach dem anschließenden Herausheben des zweiten Schichtpakets mit Hilfe der Omniprobe musste der Kontakt der beiden Seitenflächen unter besonderer Vorsicht hergestellt werden (s. Abb. 6.41 links), damit die bereits fixierte, senkrecht stehende 
Einzellinse nicht wieder von der Trägerspitze abgerissen wird. Beide Linsen wurden dabei nicht in deren Mitten, sondern an den jeweiligen Enden der Schichtsegmente verknüpft, sodass neben der Analyse des zweidimensionalen Fokus durch entsprechende Beleuchtung mit harter Röntgenstrahlung auch die vertikalen und horizontalen Einzelfoki der beiden Teillinsen untersucht werden können. Durch geringfügige Pt-Deposition an den zugänglichen Stellen der Kontaktfläche konnte sodann die Omniprobe-Nadel des FIB abgelöst und die gekreuzte MLL ausreichend mit Pt verklebt werden (s. Abb. 6.41 rechts). Das Resultat in Abbildung 6.42 zeigt schließlich die fertige Kombination zweier gekreuzter MLL zur Generierung eines zweidimensionalen Punktfokus der transmittierenden Röntgenstrahlung (durch Pfeil illustriert). Wiederum wurde die Spitze im letzten Schritt in einen speziellen, für den experimentellen Aufbau am Synchrotron-Beschleunigerring gebräuchlichen Probenhalter eingefasst. Die hier vorgestellte zweidimensionale Multischichtstruktur konnte aus zeitlichen Gründen jedoch innerhalb der letzten Synchrotron-Strahlzeit nicht mehr vermessen werden, was aber zukünftig geplant ist.
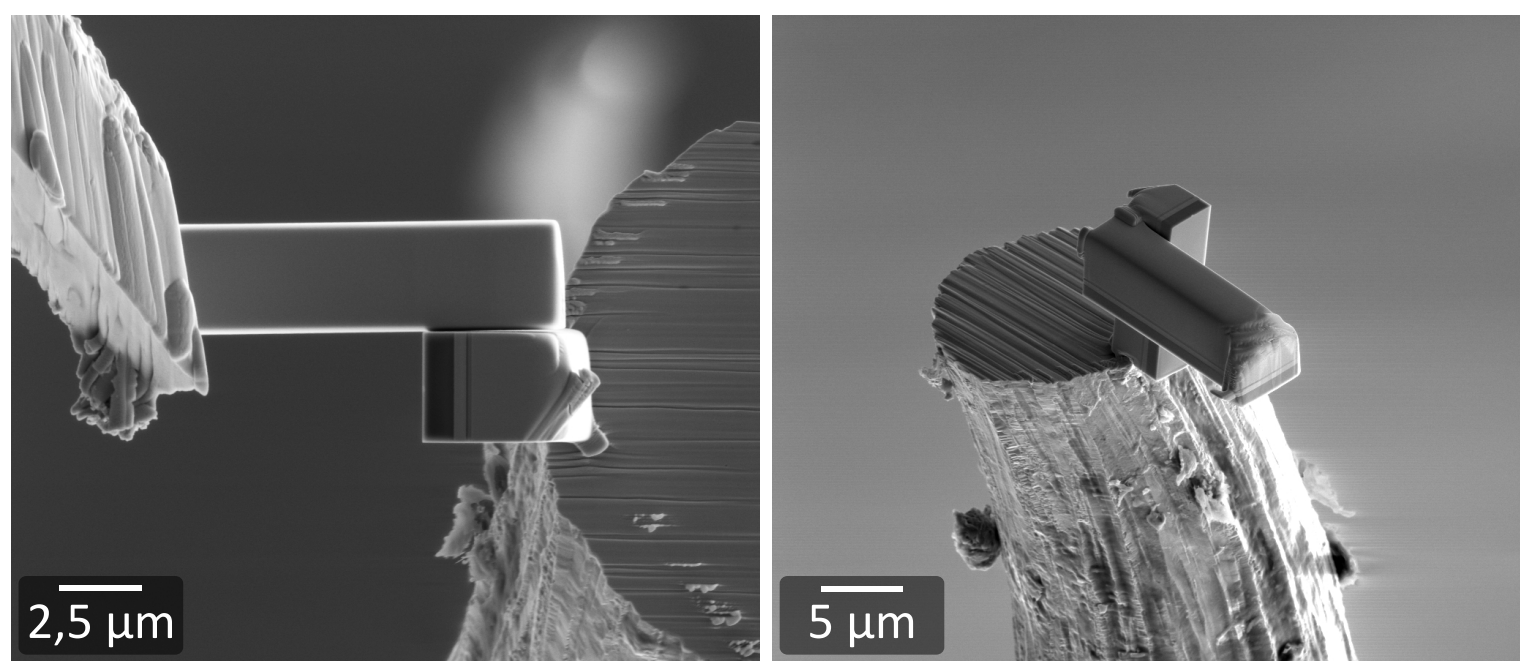

Abbildung 6.41: Aufsicht der Kontaktierung der beiden Einzellinsen an deren äußeren Enden, bei der die waagrechte zu diesem Zeitpunkt noch fest mit der Omniprobe-Nadel des FIB verbunden ist (links). Nach der Pt-Fixierung kann die Nadel abgetrennt und die gekreuzte MLL freistehend betrachtet werden (rechts). 


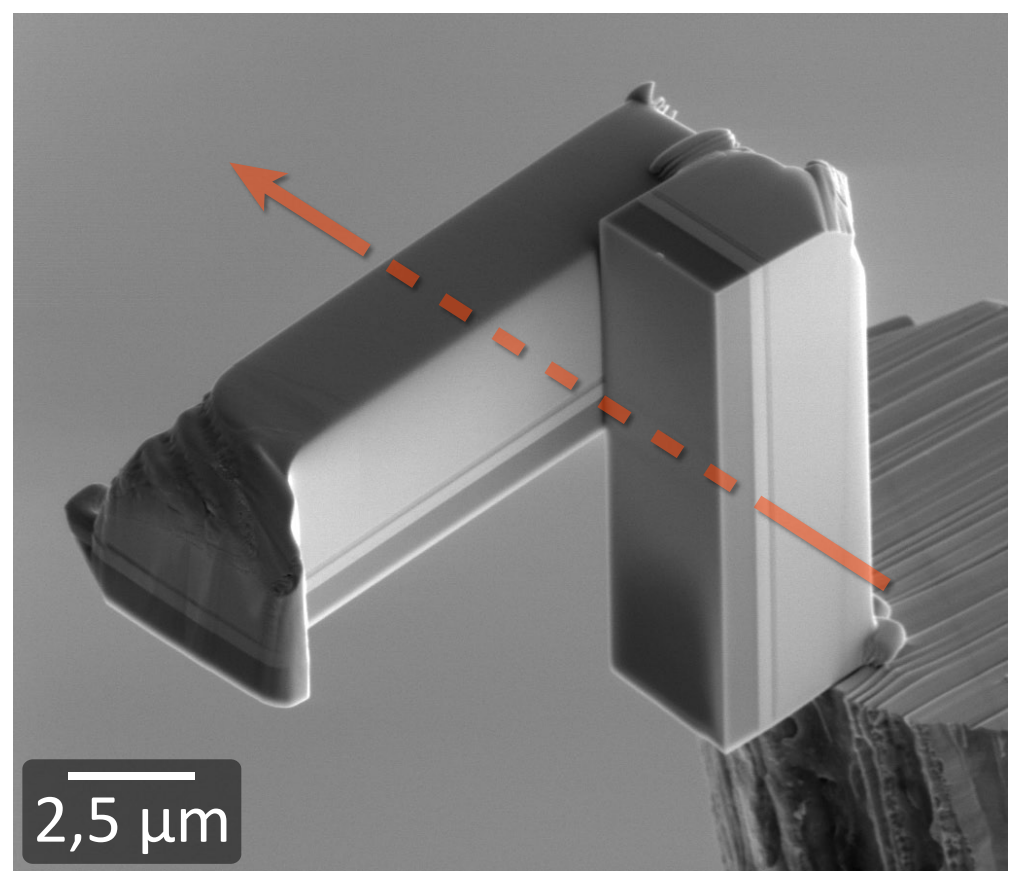

Abbildung 6.42: Die vollendete Kombination von senkrechter und waagrechter Einzellinse führt bei Röntgenbestrahlung zur Generierung eines Punktfokus. Ferner besteht die Möglichkeit, senkrechte und waagrechte Linienfoki separat zu untersuchen [185].

\subsection{Ausblick}

Außer der Untersuchung der bereits hergestellten gekreuzten MLL sind zukünftig auch MLL-Herstellungen mit verbesserten Bedingungen geplant. So könnte bei einer zweidimensionalen MLL die Fokuslänge der zweiten Linse hinsichtlich der ersten verkürzt werden, um Röntgenstrahlung tatsächlich in einem definierten Abstand punktfokussieren zu können. Dazu müssten erwartungsgemäß zwei separate, aufeinander abgestimmte Schichtpakete mit unterschiedlichen, nach dem Zonenplattenbildungsgesetz bestimmten Einzelschichtdicken hergestellt werden. Bezüglich der optimalen Wahl der optischen Tiefe $\Delta t$ einer MLL wäre es sinnvoll, nicht mit einem konstanten Wert zu arbeiten, sondern mittels FIB ein keilförmiges Ausdünnen längs eines Multischichtpakets zu erzeugen. Damit ließe sich ein variabler Phasenschub generieren, um am Synchrotron in-situ die beste Effizienz in Abhängigkeit der optischen Tiefe zu erlangen. Darüber hinaus würde eine Effizienzerhöhung mit einem lateralen Schichtdickengradient erreicht werden, da auf diese Weise eine globale Erfüllung der Bragg-Bedingung gewährleistet ist $[102,130]$. Für eine Steigerung der Effizienz könnte außerdem die Herstellung von Multilevel-MLL weiter präzisiert werden. Um für einen graduellen Phasenschub zu sorgen, könnte eine durchmischte Grenzfläche entweder durch das hier unter- 
suchte energetische Ionenmischen oder durch Legierungsherstellung mittels gezieltem Targetwechsel erzeugt werden. Um die Auflösung der Optiken weiter steigern zu können, sollen zukünftige Experimente zeigen, welche minimale auflösungsbestimmende Schichtdicken beim W/Si-System oder auch anderer Materialkombinationen mittels gepulster Laserdeposition realisierbar sind.

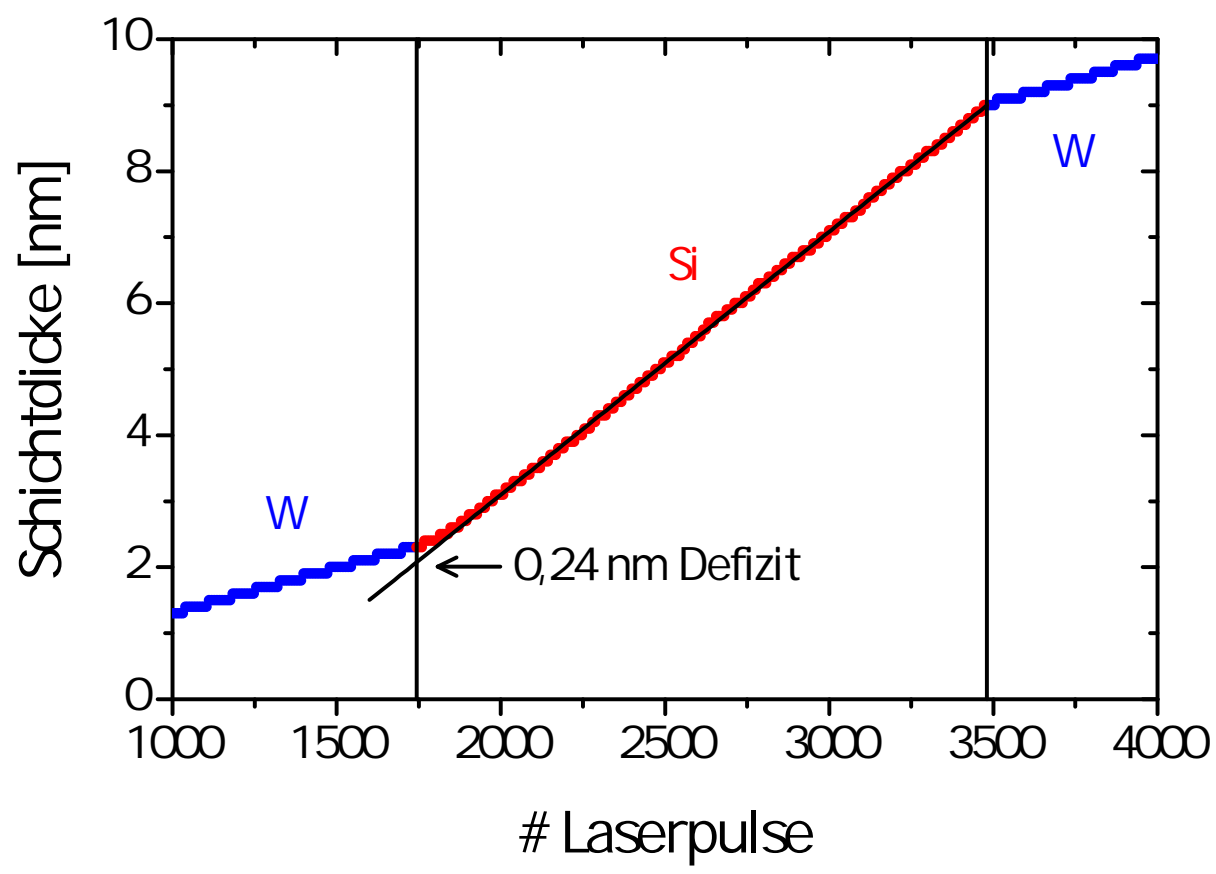

Abbildung 6.43: Untersuchung einer W/Si-Grenzfläche bei verringerten Energiedichten im Vergleich zu den verwendeten Parametern während der MLL-Herstellung. Der kaum messbare Schichtdickenverlust spricht für eine mögliche Multischichtherstellung ohne Sputtereffekte. (Die Messdaten wurden freundlicherweise von Christian Eberl zur Verfügung gestellt.)

Ebenfalls könnte die genaue Einhaltung der einzelnen Zonenbreiten einer MLL weiter präzisiert werden, indem das Absputtern von Schichtmaterial an den jeweiligen Grenzflächen vollends unterbunden wird. Dies lässt sich z. B. durch eine Reduktion der kinetischen Teilchenenergien bei optimaler Energiedichte erreichen. Geeignete Herstellungsbedingungen werden bereits im Rahmen der begleitenden Masterarbeit von Christian Eberl untersucht, bei der durch eine automatisierte Verschiebung der Fokussierungslinse des Laserstrahls für jedes Targetmaterial eine bestmögliche Energieeinkopplung definiert werden kann. Diesbezüglich machen erste Grenzflächenmessungen in Abbildung 6.43 bei verringerter Energiedichte aufgrund des sehr geringen Schichtdickenverlustes deutlich, dass eine Schichtherstellung ohne Wiederabtrag möglich ist. Auch durch das Arbeiten mit inerten Hintergrundgasen ließe sich eine Verringerung des Sputteryields infolge niedrigerer Ionenenergien erzielen [133, 205]. 
Insgesamt hat dieses Kapitel gezeigt, wie sich mittels PLD und FIB fabrizierte ein- und zweidimensionale Multilagen-Laue-Linsen zur Nanofokussierung harter Röntgenstrahlung verwenden lassen. Angefangen beim MLL-Design müssen äußere Faktoren wie Wellenlänge und Quellgröße berücksichtigt werden, um ein geeignetes Materialsystem sowie optimale Geometrien zu erarbeiten. Durch theoretische und experimentelle Untersuchungen innerer Faktoren wie Grenzflächendurchmischung und Sputtereffekte konnten in dieser Arbeit optimale Herstellungsparameter für ein präzises Multischichtwachstum gefunden werden. Mittels fokussierter Ionenstrahltechnik wurden Verbundsysteme aus eindimensionaler Optik und Probe sowie zweidimensionaler, gekreuzter Einzellinsen fabriziert, wobei erstere im Synchrotron-Experiment kombiniert mit einem KB-System eine außerordentliche Fokussierung kleiner als $7 \mathrm{~nm}$ gezeigt hat. Ergebnisse über einen kleineren Fokus sind in der Literatur bisher nicht bekannt. 



\section{Zusammenfassung}

In der hier vorliegenden Arbeit wurde das Ziel gesetzt, eine völlig neue komplementäre Methode bestehend aus gepulster Laserdeposition (PLD) und fokussierter Ionenstrahltechnik (FIB) aufzuzeigen, um multilagenbasierte Transmissionsoptiken als Alternative zu konventionellen Zonenplatten herstellen zu können. Dabei wurde vor allem auf ein physikalisches Verständnis von Ablation und Deposition (insbesondere an den Grenzflächen) unterschiedlicher aperiodischer Schichtsysteme Wert gelegt, die sich für die Herstellung entsprechender Optiken zur effizienten Fokussierung von Röntgenstrahlung im weichen und harten Wellenlängenbereich eignen.

Zum allgemeinen Verständnis der optischen Eigenschaften von Multilagen-LaueLinsen (MLL) und Multilagen-Zonenplatten (MZP) wurde zunächst die Beugungstheorie von herkömmlichen Zonenplatten betrachtet. Ihre Funktionsweise sowie theoretisch mögliche Effizienzen und Auflösungen wurden detailliert beschrieben und die Unterschiede zu MLL/MZP im Anschluss aufgeführt. In speziellen Geometrien existiert bei derartigen Multischichtoptiken kein physikalisches Limit, annähernd $70 \%$ der transmittierten Strahlung mit Auflösungen kleiner als $1 \mathrm{~nm}$ zu fokussieren. Die experimentellen Voraussetzungen für deren Herstellung ohne einen merklichen Verlust von Effizienz oder Auflösung lassen sich im Wesentlichen durch einen hohen Unterschied im Absorptionskoeffizienten der verwendeten Materialien, präzises Schichtwachstum, niedrige Grenzflächenrauigkeiten, geringe mechanische Spannungen und ausreichende Haftung zwischen Schicht und Substrat zusammenfassen. Anhand aktueller Literatur wurde anschließend der derzeitige Forschungsstand aufgeführt, an den diese Arbeit angeknüpft wurde. So waren z. B. anfangs überhaupt keine MLL oder MZP bekannt, die im Bereich weicher Röntgenstrahlung eingesetzt wurden. Im harten Röntgenwellenlängenbereich existieren bislang keine MLL, die eine Fokussierung besser als $10 \mathrm{~nm}$ zeigen.

Da es mit der ursprünglich vorhandenen Hard- und Software-Ausstattung der Depositionsanlage nicht möglich war, aperiodische Schichtpakete herzustellen, wurde 
$\mathrm{zu}$ Beginn dieser Arbeit eine komplett neue Anlagensteuerung in einer LabVIEWProgrammierumgebung entwickelt, die dem Benutzer zahlreiche neue Möglichkeiten zur Herstellung komplexer periodischer und aperiodischer Multischichtsysteme bietet. Hochpräzise Multischichten herzustellen erforderte im Laufe der Arbeit weitere Maßnahmen, die für eine experimentelle Stabilisierung der Depositionsraten ergriffen wurden. So wurde die Programmierung einer neuen Regeltechnik für eine konstante Energieeinkopplung in die Vakuumkammer (mittlere Abweichung von weniger als 0,1\%), eine Verlängerung des Strahlengangs innerhalb der Depositionsanlage sowie eine oszillierende Scheibenverschiebung (Transmissionsverlust der Laserstrahlung von $17 \%$ auf 1,5\% minimiert) und die Implementierung einer neuen Targetrotation (Minimierung von Targetaufrauung) realisiert. Weiterhin konnten die Ergebnisse automatisierter Überwachungen der Schichtherstellung dazu genutzt werden, veränderlichen Depositionsraten durch Variation der Laserenergie oder -pulszahl entgegenzuwirken.

Während der Optimierung der Herstellungsbedingungen wurde gleichzeitig mit der Fabrikation von MLL und MZP für weiche Röntgenstrahlung im Bereich des Wasserfensters $(\lambda=2,3-4,4 \mathrm{~nm})$ begonnen. Dazu wurden zunächst die Ablationsund Depositionseigenschaften geeigneter Materialsysteme $\left(\mathrm{Ti} / \mathrm{ZrO}_{2}\right.$ und $\left.\mathrm{MgO} / \mathrm{ZrO}_{2}\right)$ eingehend untersucht und passende Herstellungsparameter durch einen Kompromiss an minimaler Dropletdichten (z. B. $50 \cdot 10^{-3} / \mu \mathrm{m}^{3}$ für Ti) und ausreichender Depositionsraten (Ti: 0,0025 nm/Puls, $\mathrm{ZrO}_{2}$ : 0,0326 nm/Puls, MgO: 0,0141 nm/Puls) gefunden. Dabei ist besonders der glättende Charakter des laserdeponierten $\mathrm{ZrO}_{2}$ hervorzuheben, durch den die Multilagendeposition ohne Ausbildung einer kumulativen Rauigkeit gelingt. Besonders bei der Drahtbeschichtung erwies sich der Glättungsprozess äußerst hilfreich, um im Gegensatz zu den aus der Literatur bekannten MZP-Strukturen nahezu beliebig viele Zonen herstellen zu können. Vorhandene Substrat- oder Schichtrauigkeiten werden zudem durch den Einfluss energetischer Targetionen (bis zu $100 \mathrm{eV}$ ) effektiv ausgeglättet. Durch sensitive Schichtdickenmessungen per Depositionsratenmonitor und Röntgenreflektometrie wurde ein kontinuierliches Wachstum an den Grenzflächen der Materialsysteme ohne die Existenz von Sputtereffekten ermittelt und anschließend die Herstellung periodischer Schichtpakete mit präzise einstellbarem Schichtdickenverhältnis gezeigt.

Hergestellt wurden sodann MLL für weiche Röntgenstrahlung ( $\lambda=2,879 \mathrm{~nm})$ mit aperiodischen Schichtdicken zwischen 80 und $10 \mathrm{~nm}$, die nach dem binären Zonenplattenbildungsgesetz berechnet wurden. Die Multischichtpakete mit Gesamtschichtdicken zwischen 1 und $17 \mu \mathrm{m}$ konnten dabei ohne kumulative Rauigkeiten 
und ohne starke Verspannungen, die ein Abplatzen von den Substraten hervorrufen würden, deponiert werden. Darüber hinaus spielte die Depositionsreihenfolge (dickste oder dünnste Schicht zuerst) hinsichtlich der Grenzflächenrauigkeiten keine Rolle, was besonders für die Deposition auf rotierenden Drähten wichtig war.

Die Präparation verschiedener Transmissionsoptiken (MLL aus ein und zwei Schichtpaketen, Röntgenmonochromator) und die Untersuchungen an MZP-Demonstratoren erfolgten mit der fokussierten Ionenstrahltechnik (FIB), welche ein flexibles Schneiden, Transferieren und Polieren (minimaler Ionenstrom 30 pA) von Multischichtstrukturen unter ständiger REM-Beobachtung erlaubt. Dabei konnten die Multischichtstrukturen für die Fabrikation von Absorptions-MLL auf minimale optische Tiefen bis zu $200 \mathrm{~nm}$ gleichmäßig ausgedünnt werden. Durch in-situ Pt-Deposition wurden beam-stops erzeugt und die gefertigten Linsen auf $\mathrm{Si}_{3} \mathrm{~N}_{4}$-Trägersubstrate fixiert.

Anhand der Charakterisierung der Fokuseigenschaften einer planaren aperiodischen $\mathrm{Ti} / \mathrm{ZrO}_{2}-$ Teststruktur mittels eines Labor-Röntgenmikroskops wurde schließlich erstmals die Anwendbarkeit von MLL zur Erzeugung von Linienfoki im Bereich weicher Röntgenstrahlung mit einer Aufösung von $R=180 \mathrm{~nm}$ demonstriert. Weitere unter optimierten Bedingungen hergestellte Optiken konnten jedoch aus zeitlichen Gründen bisher noch nicht vermessen werden.

Beim Multischichtsystem W/Si wurden im darauf folgenden Teil der Arbeit zu berücksichtigende physikalische Erkenntnisse erarbeitet, um präzise Schichtdicken zu erzeugen und somit die Auflösung von MLL für harte Röntgenstrahlung voranzutreiben. Aufgrund der stark unterschiedlichen Schmelztemperaturen von W $\left(3422^{\circ} \mathrm{C}\right)$ und $\mathrm{Si}$ $\left(1414^{\circ} \mathrm{C}\right)$ waren hohe Laserenergiedichten nötig, um einen Materialabtrag insbesondere von $\mathrm{W}$ zu gewährleisten. Der daraus resultierende hohe Ionenanteil mit hohen kinetischen Energien von Si $(\approx 100 \mathrm{eV})$ führte zu einem asymmetrischen Schichtwachstum an den Grenzflächen. Wurde hochenergetisches Si auf W deponiert, ließ sich ein markanter Schichtdickenverlust bis zu 2,5 nm aufgrund von Absputtereffekten und eine sichtbare Durchmischung von W und Si im TEM bis zu $3 \mathrm{~nm}$ Dicke festhalten. SRIMund TRIDYN-Simulationen haben gezeigt, dass Si weniger stark in W implantiert wird als umgekehrt $\left(\bar{x}_{\mathrm{Si} \rightarrow \mathrm{W}}=0,6 \mathrm{~nm}, \bar{x}_{\mathrm{W} \rightarrow \mathrm{Si}}=2,5 \mathrm{~nm}\right.$ bei $\left.E_{k i n}=100 \mathrm{eV}\right)$, sodass das eigentliche Schichtwachstum nur wenige Monolagen unterhalb der Oberfläche stattfindet („subsurface growth“). Während dieses Ionenmischens wird W nahe an die Schichtoberfläche geschoben, sodass der Effekt einer Verstärkung des Sputteryields und folglich ein Defizit an deponiertem Material auftritt. Nur aus dem hier gewonnenen Verständnis der Wachstumseigenschaften heraus konnte bei vorbereitenden Beschich- 
tungsprozessen für die MLL-Herstellung der Schichtdickenverlust durch eine entsprechende Addition an Laserpulsen ausgeglichen und genaue Schichtdickenverhältnisse (a)periodischer Multischichten eingestellt werden.

Mit einem aperiodischen W/Si-Schichtpaket konnte sodann im FIB eine eindimensionale MLL für harte Röntgenstrahlung $(\lambda=0,0898 \mathrm{~nm})$ mit Schichtdicken zwischen 36 und 2,6 nm fabriziert werden. Die gesamte Multischicht (400 nm Dicke) wurde anschließend im FIB auf $3,26 \mu \mathrm{m}$ optische Tiefe ausgedünnt, um eine Phasenschub von $\pi \mathrm{zu}$ erzeugen. Weiterhin wurde eine demonstrative zweidimensionale MLL hergestellt, indem zwei Schichtsegmente in gekreuzter Geometrie durch Pt-Fixierung miteinander verbunden wurden. Der wesentliche Vorteil einer solchen in sich vibrationsfreien Anordnung ist in dem Wegfall aufwändiger Justageeinheiten und -arbeiten zu sehen, um zwei gekreuzte Einzellinsen zueinander auszurichten. In einer innovativen Kombination mit einem vorfokussierenden Kirkpatrick-Baez-Spiegel konnte anhand einer mit Synchrotronstrahlung durchgeführten Analyse der eindimensionalen MLL eine Fokusgröße von nur 6,8 nm ermittelt werden. In der aktuellen Forschung stellt diese die bisher beste erreichte Aufösung einer Zonenplattenoptik im Bereich harter Röntgenstrahlung dar.

Insgesamt konnte in dieser Arbeit also gezeigt werden, dass sich ein- und zweidimensionale Multilagen-Laue-Linsen und Multilagen-Zonenplatten auf innovative Weise mittels gepulster Laserdeposition und fokussierter Ionenstrahltechnik herstellen lassen. Diese vielversprechende Methode eröffnet neue physikalische und technologische Wege zur Nanofokussierung weicher und harter Röntgenstrahlung für die Anwendung hochauflösender Röntgenmikroskopie. 


\section{Literaturverzeichnis}

[1] Röntgen W. C.: Ueber eine neue Art von Strahlen. Sitzungsberichte der physikal.medizin. Gesellschaft zu Würzburg (1895)

[2] Schmahl G., Rudolph D., Niemann B., Guttmann P., Thieme J., Schneider G.: Röntgenmikroskopie. Naturwissenschaften 83 (1996), S. 61

[3] Bauer A.: Röntgenmikroskopie. Physik in unserer Zeit 23 (1992), S. 151

[4] Kirz J., Jacobsen C., Howells M.: Soft X-ray microscopes and their biological applications. Quarterly Reviews of Biophysics 28 (1995), S. 33

[5] Howells M., Jacobsen C., Warwick T., Bos A.: Principles and Applications of Zone Plate X-Ray Microscopes. In: Hawkes P. W. (Hrsg.), Spence J. C. H. (Hrsg.): Science of Microscopy. Springer New York 2007 S. 835

[6] Kinz J., Jacobsen C.: The history and future of X-ray microscopy. J. Phys.: Conf. Series: 9th International Conference On X-Ray Microscopy 186 (2009), S. 012001

[7] Miao J., Charalambous P., Kirz J., Sayre D.: Extending the methodology of X-ray crystallography to allow imaging of micrometre-sized non-crystalline specimens. Nature 400 (1999), S. 342

[8] Shapiro D., Thibault P., Beetz T., Elser V., Howells M., Jacobsen C., Kirz J., Lima E., Miao H., Neiman A. M., Sayre D.: Biological imaging by soft x-ray diffraction microscopy. PNAS 102 (2005), S. 15343

[9] Holzner C., Feser M., Vogt S., Hornberger B., Baines S. B., Jacobsen C.: Zernike phase contrast in scanning microscopy with X-rays. Nature Physics 6 (2010), S. 883

[10] Atтwood D.: Nanotomography comes of age. Nature 442 (2006), S. 642

[11] Schneider G., Guttmann P., Heim S., Rehbein S., Mueller F., Nagashima K., Heymann J. B., Müller W. G., McNally J. G.: Three-dimensional cellular ultrastructure resolved by X-ray microscopy. Nature Methods 7 (2010), S. 985

[12] Sakdinawat A., Attwood D.: Nanoscale X-ray imaging. Nature Photonics 4 (2010), S. 840 
[13] Hell S. W., Wichmann J.: Breaking the diffraction resolution limit by stimulated emission: stimulated-emission-depletion fluorescence microscopy. Opt. Lett. 19 (1994), S. 780

[14] Aquino D., Schonle A., Geisler C., von Middendorff C., Wurm C. A., Okamura Y., Lang T., Hell S. W., Egner A.: Two-color nanoscopy of threedimensional volumes by 4 Pi detection of stochastically switched fluorophores. Nature Methods 8 (2011), S. 353

[15] Robinson A. L.: History of Synchrotron Radiation. In: Thompson A. C. (Hrsg.): X-Ray Data Booklet. Center for X-ray Optics and Advanced Light Source (2009) S. 2

[16] Takman P. A. C., Stollberg H., Johansson G. A., Holmberg A., Lindblom M., Hertz H. M.: High-resolution compact X-ray microscopy. Journal of Microscopy 226 (2007), S. 175

[17] Kirkpatrick P., Baez A. V.: Formation of Optical Images by X-Rays. J. Opt. Soc. Am. 38 (1948), S. 773

[18] Mimura H., Yumoto H., Matsuyama S., Sano Y., Yamamura K., Mori Y., Yabashi M., Nishino Y., Tamasaku K., Ishikawa T., Yamauchi K.: Efficient focusing of hard x-rays to $25 \mathrm{~nm}$ by a total reflection mirror. Appl. Phys. Lett. 90 (2007), S. 051903

[19] Spiller E., Segmüller A.: Propagation of x-rays in waveguides. Appl. Phys. Lett. 24 (1974), S. 60

[20] Krüger S. P., Neubauer H., Bartels M., Kalbfleisch S., Giewekemeyer K., Wilbrandt P. J., Sprung M., Salditt T.: Sub-10nm beam confinement by $X$ ray waveguides: design, fabrication and characterization of optical properties. J. Synchrotron Radiat. 19 (2012), S. 227

[21] Snigirev A., Kohn V., Snigireva I., Lengeler B.: A compound refractive lens for focusing high-energy X-rays. Nature 384 (1996), S. 49

[22] Schroer C. G., Kurapova O., Patommel J., Boye P., Feldkamp J., Lengeler B., Burghammer M., Riekel C., Vincze L., van der Hart A., Küchler M.: Hard x-ray nanoprobe based on refractive $x$-ray lenses. Appl. Phys. Lett. 87 (2005), S. 124103

[23] Niemann B., Rudolph D., Schmahl G.: Soft X-ray imaging zone plates with large zone numbers for microscopic and spectroscopic applications. Opt. Commun. 12 (1974), S. 160 
[24] Chao W., Kim J., Rekawa S., Fischer P., Anderson E. H.: Demonstration of 12 nm Resolution Fresnel Zone Plate Lens based Soft X-ray Microscopy. Opt. Express 17 (2009), S. 17669

[25] Rehbein S., Guttmann P., Werner S., Schneider G.: Soft X-Ray Microscopy at HZB: Zone Plate Development and Imaging Using the Third Order of Diffraction. AIP Conf. Proc.: 10th International Conference on X-Ray Microscopy 1365 (2011), S. 32

[26] Vila-Comamala J., Gorelick S., Färm E., Kewish C. M., Diaz A., Guzenko V. A., Barrett R., RaAbe J., Menzel A., Bunk O., Ritala M., David C.: ZoneDoubled Fresnel Zone Plates for Scanning Transmission X-ray Microscopy. AIP Conf. Proc.: 10th International Conference on X-Ray Microscopy 1365 (2011), S. 192

[27] Robinson A. L.: High-resolution imaging with soft x-rays. Science 215 (1982), S. 150

[28] Schmahl G.: X-ray microscopy. Nucl. Instrum. Meth. Phys. Res. 208 (1983), S. 361

[29] Schmahl G., Rudolph D., Lengeler B., Schroer C.: Röntgenlinsen. In: Physikalische Blätter Bd. 57, Nr. 12001 S. 43

[30] Maser J., Stephenson G. B., Vogt S., Yun W., Macrander A. T., Kang H. C., LiU C., Conley R.: Multilayer Laue lenses as high-resolution x-ray optics. SPIE Conf. Proc.: Design and Microfabrication of Novel X-Ray Optics II 5539 (2004), S. 185

[31] Liu C., Conley R., Macrander A. T., Maser J., Kang H. C., Zurbuchen M. A., Stephenson G. B.: Depth-graded multilayers for application in transmission geometry as linear zone plates. J. Appl. Phys. 98 (2005), S. 113519

[32] Schmahl G.: Röntgenoptik. In: Bergmann, Schäfer: Lehrbuch der Experimentalphysik, Band 3: Optik; Walter der Gruyter, Berlin - New York 2004

[33] Attwood D.: Soft x-rays and extreme ultraviolet radiation. Cambridge University Press 1999

[34] Schmahl G., Rudolph D.: X-ray microscopy. Springer-Verlag, Berlin 1984

[35] Thieme J., Schmahl G., Umbach E.: X-ray microscopy and spectromicroscopy. Springer-Verlag, Heidelberg 1998

[36] SoRet J. L.: Über die von Kreisgittern erzeugte Diffraktionsphänomene. Annalen der Physik und Chemie 156 (1875), S. 99

[37] Lord Rayleigh: Wave theory of light. Encyclopaedia Britannica, Ninth Edition, Volume 24 p. 429 (1889) 
[38] David C., Thieme J., Guttmann P., Schneider G., Rudolph D., Schmahl G.: Electron-beam generated $x$-ray optics for high-resolution microscopy studies. Optik 91 (1992), S. 95

[39] KIRz J.: Phase zone plates for x-rays and the extreme uv. J. Opt. Soc. Am. 64 (1974), S. 301

[40] CXRO: Center for X-Ray Optics, Lawrence Berkeley National Laboratory

[41] Di Fabrizio E., Romanato F., Gentili M., Cabrini S, Kaulich B., Susini J., BARrett R.: High-efficiency multilevel zone plates for keV X-rays. Nature 401 (1999), S. 895

[42] Jahns J., Walker S. J.: Two-dimensional array of diffractive microlenses fabricated by thin film deposition. Appl. Opt. 29 (1990), S. 931

[43] Maser J., Schmahl G.: Coupled wave description of the diffraction by zone plates with high aspect ratios. Opt. Comm. 89 (1992), S. 355

[44] Schroer C.: Focusing hard x-rays to nanometer dimensions using Fresnel zone plates. Phys. Rev. B 74 (2006), S. 033405

[45] Jacobsen C., Kirz J., Williams S.: Resolution in soft X-ray microscopes. Ultramicroscopy 47 (1992), S. 55

[46] Pfeiffer F., David C., van der Veen J. F., Bergemann C.: Nanometer focusing properties of Fresnel zone plates described by dynamical diffraction theory. Phys. Rev. B 73 (2006), S. 245331

[47] Bergemann C., Keymeulen H., van der Veen J. F.: Focusing X-Ray Beams to Nanometer Dimensions. Phys. Rev. Lett. 91 (2003), S. 204801

[48] Schmahl G., Rudolph D., Niemann B.: X-ray Microscopy Using Fresnel Zone Plates. AIP Conf. Proc. 75 (1981), S. 225

[49] Jochum L., Niemann B.: X-Ray Microscopy with Synchrotron Radiation and 3-Dimensional Image Data Processing. Science on Form: Proceedings of the Second International Symposium for Science on Form (1990), S. 115

[50] Schmahl G., Rudolph D., Schneider G., Thieme J., Schliebe T., Kaulich B., Hettwer M.: Diffraction optics for X-ray imaging. Microelectron. Eng. 32 (1996), S. 351

[51] Ilinski P. P., Lai B. P., Bassom N. J., Donald J., Athas G. J.: X-ray zone plate fabrication using a focused ion beam. SPIE Conf. Proc.: Advances in X-Ray Optics 4145 (2001), S. 311 
[52] Surpi A., Valizadeh S., Leifer K., Lagomarsino S.: Focused ion beam fabrication procedures of x-ray micro Fresnel zone plates. J. Micromech. Microeng. 17 (2007), S. 617

[53] Lenz J., Krupp N., Wilhein T., Irsen S.: Nanofabrication of Optical Elements for SXR and EUV Applications: Ion Beam Lithography as a New Approach. AIP Conf. Proc.: 10th International Conference on X-Ray Microscopy 1365 (2011), S. 104

[54] Matthies T., David C., Thieme J.: Trilevel reactive ion etching processes for fabrication of $60 \mathrm{~nm}$ germanium structures with high aspect ratio. J. Vac. Sci. \& Tech. B 11 (1993), S. 1873

[55] David C., Kaulich B., Medenwaldt R., Hettwer M., Fay N., Diehl M., Thieme J., Schmahl G.: Low-distortion electron-beam lithography for fabrication of highresolution germanium and tantalum zone plates. J. Vac. Sci. Technol. B 13 (1995), S. 2762

[56] Simpson M. J., Michette A. G.: The Effects of Manufacturing Inaccuracies on the Imaging Properties of Fresnel Zone Plates. Optica Acta 30 (1983), S. 1455

[57] Hambach D., Peuker M., Schneider G.: Nanostructured diffractive optical devices for soft X-ray microscopes. Nucl. Instrum. Meth. Phys. Res. A 467 (2001), S. 877

[58] Holmberg A., Lindblom M., Reinspach J., Bertilsson M., Hertz H. M.: Soft xray zone plate fabrication at KTH, Stockholm. J. Phys. Conf. Series: 9th International Conference On X-Ray Microscopy 186 (2009), S. 012065

[59] Lindblom M., Reinspach J., von Hofsten O., Bertilson M., Hertz H. M., Holmberg A.: High-aspect-ratio germanium zone plates fabricated by reactive ion etching in chlorine. J. Vac. Sci. Technol. B 27 (2009), S. L1

[60] Vila-Comamala J., Jefimovs K., Pilvi T., Ritala M., Sarkar S. S., Solak H. H., Guzenko V. A., Stampanoni M., Marone F., Raabe J., Tzvetkov G., Fink R. H., Grolimund D., Borca C. N., Kaulich B., David C.: Advanced X-ray diffractive optics. J. Phys.: Conf. Series: 9th International Conference on X-Ray Microscopy 186 (2009), S. 012078

[61] David C., Weitkamp T., Nöhammer B., van der Veen J. F.: Diffractive and refractive X-ray optics for microanalysis applications. Spectrochimica Acta Part B: Atomic Spectroscopy 59 (2004), S. 1505

[62] Feng Y., Feser M., Lyon A., Rishton S., Zeng X., Chen S., Sassolini S., Yun W.: Nanofabrication of high aspect ratio $24 \mathrm{~nm}$ x-ray zone plates for $x$-ray imaging applications. 25 (2007), S. 2004 
[63] Lindblom M., Reinspach J., von Hofsten O., Bertilson M., Hertz H. M., HolmBerG A.: Nickel-germanium soft x-ray zone plates. J. Vac. Sci. Technol. B 27 (2009), S. L5

[64] Reinspach J., Lindblom M., Bertilson M., von Hofsten O., Hertz H. M., HolmberG A.: $13 \mathrm{~nm}$ high-efficiency nickel-germanium soft $x$-ray zone plates. J. Vac. Sci. Technol. B 29 (2011), S. 011012

[65] Holmberg A., Reinspach J., Lindblom M., Chubarova E., Bertilson M., von Hofsten O., Nilsson D., Selin M., Larsson D., Skoglund P., Lundström U., Takman P., Vogt U., Hertz H. M.: Towards 10-nm Soft X-Ray Zone Plate Fabrication. AIP Conf. Proc.: 10th International Conference on X-Ray Microscopy 1365 (2011), S. 18

[66] Werner S., Rehbein S., Guttman P., Heim S., Schneider G.: Towards stacked zone plates. J. Phys.: Conf. Series: 9th International Conference on X-Ray Microscopy 186 (2009), S. 012079

[67] Rehbein S., Schneider G.: Volume Zone Plate Development at BESSY. IPAP Conf. Series: Proceedings of the 8th International Conference on X-Ray Microscopy 7 (2006), S. 103

[68] Chao W., Harteneck B. D., Liddle J. A., Anderson E. H., Attwood D. T.: Soft $X$-ray microscopy at a spatial resolution better than $15 \mathrm{~nm}$. Nature 435 (2005), S. 10210

[69] Simpson M.J., Michette A.G.: Imaging Properties of Modified Fresnel Zone Plates. Optica Acta 31 (1984), S. 403

[70] von Hofsten O., Bertilson M., Reinspach J., Holmberg A., Hertz H. M., Vogt U.: Sub-25-nm laboratory x-ray microscopy using a compound Fresnel zone plate. Opt. Lett. 34 (2009), S. 2631

[71] Jefimovs K., Vila-Comamala J., Pilvi T., Raabe J., Ritala M., David C.: ZoneDoubling Technique to Produce Ultrahigh-Resolution X-Ray Optics. Phys. Rev. Lett. 99 (2007), S. 264801

[72] Vila-Comamala J., Gorelick S., Färm E., Kewish C. M., Diaz A., Barrett R., Guzenko V. A., Ritala M., David C.: Ultra-high resolution zone-doubled diffractive X-ray optics for the multi-keV regime. Opt. Express 19 (2011), S. 175

[73] Yin G.-C., Song Y.-F., Tang M.-T., Chen F.-R., Liang K. S., Duewer F. W., FeSER M., Yun W., ShieH H.-P. D.: $30 \mathrm{~nm}$ resolution $x$-ray imaging at $8 \mathrm{keV}$ using third order diffraction of a zone plate lens objective in a transmission microscope. Appl. Phys. Lett. 89 (2006), S. 221122 
[74] Rehbein S., Heim S., Guttmann P., Werner S., Schneider G.: UltrahighResolution Soft-X-Ray Microscopy with Zone Plates in High Orders of Diffraction. Phys. Rev. Lett. 103 (2009), S. 110801

[75] Koyama T., Takenaka H., Ichimaru S., Ohchi T., Tsuji T., Takano H., Kagoshima Y.: Development of Multilayer Laue Lenses; (1) Linear Type. AIP Conf. Proc.: The 10th International Conference On X-Ray Microscopy 1365 (2011), S. 24

[76] Kamijo N., Tamura S., Suzuki Y., Kinara H.: Fabrication and testing of hard x-ray sputtered-sliced zone plate. Rev. Sci. Instrum. 66 (1995), S. 2132

[77] Kamijo N., Tamura S., Suzuki Y., Handa K., Takeuchi A., Yamamoto S., Ando M., Ohsumi K., Kinara H.: Fabrication of a hard $x$-ray sputtered-sliced Fresnel phase zone plate. Rev. Sci. Instrum. 68 (1997), S. 14

[78] Tamura S., Murai K., Kamijo N., Yoshida K., Kihara H., Suzuki Y.: Focusing efficiency of a multilayer Fresnel zone plate for hard X-ray fabricated by DC sputtering deposition. Vacuum 59 (2000), S. 553

[79] Awaji M., Suzuki Y., Takeuchi A., Takano H., Kamijo N., Tamura S., Yasumoto M.: X-ray imaging microscopy at $25 \mathrm{keV}$ with Fresnel zone plate optics. Nucl. Instrum. Meth. Phys. Res. A 467 (2001), S. 845

[80] Kamijo N., Suzuki Y., Awaji M., Takeuchi A., Uesugi K., Yasumoto M., Tamura S., Kohmura Y., Duevel A., Rudolph D., Schmahl G.: Characterization of the sputtered-sliced zone plate for high energy X-rays. Nucl. Instrum. Meth. Phys. Res. A 467 (2001), S. 868

[81] Kamijo N., Suzuki Y., Takano H., Tamura S., Yasumoto M., Takeuchi A., Awaji M.: Microbeam of $100 \mathrm{keV} \mathrm{X-ray} \mathrm{with} \mathrm{a} \mathrm{Sputtered-Sliced} \mathrm{Fresnel} \mathrm{Zone} \mathrm{Plate.} \mathrm{Rev.} \mathrm{Sci.}$ Instrum. 74 (2003), S. 5101

[82] Koyama T., Takano H., Konishi S., Tsuji T., Takenaka H., Ichimaru S., Ohchi T., Kagoshima Y.: Circular multilayer zone plate for high-energy $x$-ray nano-imaging. Rev. Sci. Instrum. 83 (2012), S. 013705

[83] Saitoh K., Inagawa K., Kohra K., Hayashi C., Lida A., Kato N.: Characterization of sliced multilayer zone plates for hard x-rays. Rev. Sci. Instrum. 60 (1989), S. 1519

[84] Yun W. B., Viccaro P. J., Lai B., Chrzas J.: Coherent hard x-ray focusing optics and applications. Rev. Sci. Instrum. 63 (1992), S. 582

[85] Yan H., Maser J., Macrander A. T., Shen Q., Vogt S., Stephenson G. B., Kang H. C.: Takagi-Taupin description of $x$-ray dynamical diffraction from diffractive optics with large numerical aperture. Phys. Rev. B 76 (2007), S. 115438 
[86] Koyama T., Ichimaru S., Tsuji T., Takano H., Kagoshima Y., Ohchi T., Takenaka H.: Optical Properties of $\mathrm{MoSi}_{2} / \mathrm{Si}$ Multilayer Laue Lens as Nanometer X-ray Focusing Device. Appl. Phys. Express 1 (2008), S. 117003

[87] Kamijo N., Suzuki Y., Tamura S., Takeuchi A., Yasumoto M.: Practical Use of Quasi-Kinoform Zone Plate: Towards High-Efficiency Microbeam for Hard/HighEnergy X-Rays. IPAP Conf. Series: Proceedings of the 8th International Conference on X-Ray Microscopy 7 (2006), S. 97

[88] Yasumoto M., Tamura S., Kamijo N., Suzuki Y., Takeuchi A.: Diffraction Efficiency of Multi-Level Zone Plate Fabricated by Sputtered-Sliced Method for Hard $X$-Ray Focusing. IPAP Conf. Series: Proceedings of the 8th International Conference on X-Ray Microscopy 7 (2006), S. 119

[89] Kang H. C., Maser J., Stephenson G. B., Liu C., Conley R., Macrander A. T., Vogt S.: Nanometer Linear Focusing of Hard X-Rays by a Multilayer Laue Lens. Phys. Rev. Lett. 96 (2006), S. 127401

[90] Yan H., Kang H. C., Conley R., Liu C., Macrander A. T., Stephenson G. B., Maser J.: Multilayer Laue Lens: A Path Toward One Nanometer X-Ray Focusing. X-Ray Optics and Instrumentation 2010 (2010)

[91] SCHNEIDER G.: Influence of roughness and interdiffusion in zone plate structures on the diffraction efficiency described by coupled wave theory. Appl. Phys. Lett. 73 (1998), S. 599

[92] YAN H.: X-ray dynamical diffraction from multilayer Laue lenses with rough interfaces. Phys. Rev. B 79 (2009), S. 165410

[93] Liu Chian, Conley R., Macrander A. T., Maser J., Kang H.C., Stephenson G.B.: A multilayer nanostructure for linear zone-plate applications. Thin Solid Films 515 (2006), S. 654

[94] Yan H., Maser J., Kang H. C., Macrander A. T., Stephenson B.: A theoretical study of two-dimensional point focusing by two multilayer Laue lenses. SPIE Conf. Proc.: Advances in X-Ray/EUV Optics and Components III 7077 (2008), S. 70770Q

[95] Yan H., Kang H. C., Maser J., Macrander A. T., Kewish C. M., Liu C., Conley R., Stephenson G. B.: Characterization of a multilayer Laue lens with imperfections. Nucl. Instrum. Meth. Phys. Res. 582 (2007), S. 126

[96] Hart H. E., Scrandis J. B., Mark R., Hatcher R. D.: Diffraction Characteristics of a Linear Zone Plate. J. Opt. Soc. Am. 56 (1966), S. 1018 
[97] Rudolph D., Niemann B., Schmahl G.: Status Of The Sputtered Sliced Zone Plates For X-Ray Microscopy. Proc. SPIE 316 (1981), S. 103

[98] Bionta R. M., Skulina K. M., Weinberg J.: Hard x-ray sputtered-sliced phase zone plates. Appl. Phys. Lett. 64 (1994), S. 945

[99] Awaji M., Suzuki Y., Takeuchi A., Takano H., Kamijo N., Yasumoto M., Terada Y., TAmura S.: Microfocusing of $82 \mathrm{keV}$ x-rays with a sputtered-sliced Fresnel zone plate. Rev. Sci. Instrum. 74 (2003), S. 4948

[100] Suzuki Y., Takeuchi A., Uesugi K., Awaji M., Yasumoto M., Tamura S., Kamijo N.: X-Ray Imaging Microscopy at 82 keV with Sputtered-Sliced Fresnel Zone Plate Objective. IPAP Conf. Series: Proceedings of the 8th International Conference on XRay Microscopy 7 (2006), S. 47

[101] Kamijo N., Suzuki Y., Takeuchi A., Itou M., Tamura S.: Microbeam of $200 \mathrm{keV}$ X-ray with Sputtered-Sliced Zone Plate. Jpn. J. Appl. Phys. 48 (2009), S. 010219

[102] Koyama T., Tsuji T., Takano H., Kagoshima Y., Ichimaru S., Ohchi T., Takenaka H.: Development of Multilayer Laue Lenses; (2) Circular Type. AIP Conf. Proc.: The 10th International Conference On X-Ray Microscopy 1365 (2011), S. 100

[103] Tamura S., Yasumoto M., Kamijo N., Awaji M., Takeuchi A., Uesugi K., Terada Y., Suzuki Y., Takano H.: Multilayer Fresnel Zone Plate with High Diffraction Efficiency for High Energy X-Ray Region at AIST and SPring-8. IPAP Conf. Series: Proceedings of the 8th International Conference on X-Ray Microscopy 7 (2006), S. 107

[104] Tamura S., Yasumoto M., Kamijo N., Uesugi K., Takeuchi A, Terada Y., SUZUKI Y.: Quasi-kinoform type multilayer zone plate with high diffraction efficiency for high-energy X-rays. J. Phys.: Conf. Series: 9th International Conference on X-Ray Microscopy 186 (2009), S. 012075

[105] Tamura S.: Multilayer Fresnel Zone Plate with High-Diffraction Efficiency: Application of Composite Layer to X-Ray Optics. In: Cuppoletti J. (Hrsg.): Metal, Ceramic and Polymeric Composites for Various Uses. InTech 2011 S. 637

[106] Mayer M., Grévent C., Szeghalmi A., Knez M., Weigand M., Rehbein S., Schneider G., BAREtzky B., Schütz G.: Multilayer Fresnel zone plate for soft X-ray microscopy resolves sub-39 nm structures. Ultramicroscopy 111 (2011), S. 1706

[107] Golant K. M., Lavrishchev S. V., Popov A. V., Artyukov I. A., Feshchenko R. M., Mitrofanov A. N., Vinogradov A. V.: Fabrication of x-ray zone plates by surface-plasma chemical vapor deposition. Appl. Opt. 46 (2007), S. 5964 
[108] Artyukov I. A., Bukreeva I. N., Chernov V. A., Feshchenko R. M., Golant K. M., Jark W., Lavrishchev S. V., Mitrofanov A. N., Popov A. V., Vinogradov A. V.: Zone plates for hard X-rays fabricated with the SPCVD technology. Nucl. Instrum. Meth. Phys. Res. A 603 (2009), S. 66

[109] Fullerton E. E., Pearson J., Sowers C. H., Bader S. D., Wu X. Z., Sinha S. K.: Interfacial roughness of sputtered multilayers: Nb/Si. Phys. Rev. B 48 (1993), S. 17432

[110] Salditt T., Metzger T. H., Peisl J.: Kinetic Roughness of Amorphous Multilayers Studied by Diffuse X-Ray Scattering. Phys. Rev. Lett. 73 (1994), S. 2228

[111] Salditt T., Lott D., Metzger T. H., Peisl J., Vignaud G., Høghøu P., Schärpf O., Hinze P., LAUeR R.: Interfacial roughness and related growth mechanisms in sputtered W/Si multilayers. Phys. Rev. B 54 (1996), S. 5860

[112] Ghafoor N., Persson P. O. A., Birch J., Eriksson F., Schäfers F.: Interface engineered ultrashort period Cr-Ti multilayers as high reflectance mirrors and polarizers for soft $x$-rays of $\lambda=2,74$ nm wavelength. Appl. Opt. 45 (2006), S. 137

[113] Wang Y.-P., Zhou H., Zhou L., Headrick R. L., Macrander A. T., Özcan A. S.: Interface roughness evolution in sputtered WSi2/Si multilayers. J. Appl. Phys. 101 (2007), S. 023503

[114] Thornton J. A.: The microstructure of sputter-deposited coatings. J. Vac. Sci. Technol. A 4 (1986), S. 3059

[115] Tamura S., Yasumoto M., Mihara T., Kamijo N., Suzuki Y., Awaji M., Takeuchi A., Takano H., Handa K.: Multilayer Fresnel zone plate for high-energy X-ray by DC sputtering deposition. Vacuum 66 (2002), S. 495

[116] Tamura S., Ohtani K., KamiJo N.: Materials for multilayer zone plates: development of a focusing element for use in SR photo-excited processes. Appl. Surf. Sci. 79 (1994), S. 514

[117] Düvel A., Rudolph D., Schmahl G.: Fabrication of thick zone plates for multikilovolt X-rays. AIP Conf. Proc.: 6th International Conference on X-Ray Microscopy 507 (2000), S. 607

[118] Bionta R. M.: Transmission gratings that diffract 8 keV x-rays. Appl. Phys. Lett. 51 (1987), S. 725

[119] Koike M., Suzuki I.H.: Linear bragg fresnel x-ray optical device using a multilayer zone plate and a crystal. In: Pacific Rim Conference on Lasers and Electro-Optics 1995 S. 106 
[120] Koike M, Suzuki I H., Komiya S, Amemiya Y: Ti/Al multilayer zone plate and Bragg-Fresnel lens. J. Synchrotron Radiat. 5 (1998), S. 794

[121] Kang H. C., Stephenson G. B., Liu C., Conley R., Khachatryan R., Wieczorek M., Macrander A. T., Yan H., Maser J., Hiller J., Koritala R.: Sectioning of multilayers to make a multilayer Laue lens. Rev. Sci. Instrum. 78 (2007), S. 046103

[122] Bouet N., Conley R., Biancarosa J., Divan R., Macrander A. T.: WSi $/$ Si multilayer sectioning by reactive ion etching for multilayer Laue lens fabrication. SPIE Conf. Proc.: Advances in X-Ray/EUV Optics and Components V 7802 (2010), S. 780203

[123] Liu C., Conley R., Qian J., Kewish C. M., Macrander A. T., Maser J., Kang H. C., Yan H., Stephenson G. B.: Bonded Multilayer Laue Lens for focusing hard X-rays. Nucl. Instrum. Meth. Phys. Res. A 582 (2007), S. 123

[124] Takenaka H., Ichimaru S., Ohchi T., Koyama T., Tsuji T., Takano H., Kagoshima Y.: Mo/Si and MoSi $/$ Si nanostructures for multilayer Laue lens. J. Phys.: Conf. Series: 9th International Conference on X-Ray Microscopy 186 (2009), S. 012074

[125] Kang H. C., Yan H., Winarski R. P., Holt M. V., Maser J., Liu C., Conley R., Vogt S., Macrander A. T., Stephenson G. B.: Focusing of hard $x$-rays to 16 nanometers with a multilayer Laue lens. Appl. Phys. Lett. 92 (2008), S. 221114

[126] Yan H., Rose V., Shu D., Lima E., Kang H. C., Conley R., Liu C., Jahedi N., Macrander A. T., Stephenson G. B., Holt M., Chu Y. S., Lu M., Maser J.: Two dimensional hard $x$-ray nanofocusing with crossed multilayer Laue lenses. Opt. Express 19 (2011), S. 15069

[127] Shu D., MASER J.: Mechanical design of ultraprecision weak-link stages for nanometerscale $x$-ray imaging. J. Phys.: Conf. Series: 9th International Conference on X-Ray Microscopy 186 (2009), S. 012017

[128] Shu D., Maser J., Chu Y., Yan H., Nazaretski E., O’Hara S., Kearney S., Anton J., Quintana J., Shen Q.: Development of an Advanced Sample-Scanning Stage System Prototype for an MLL-Based Hard X-ray Nanoprobe. AIP Conf. Proc.: 10th International Conference on X-Ray Microscopy 1365 (2011), S. 144

[129] Conley R., Bouet N., Lauer K., Carlucci-Dayton M., Biancarosa J., Boas L., Drannbauer J., Feraca J., Rosenbaum L.: Multilayer Laue Lens Growth at NSLS-II. AIP Conf. Proc.: 10th International Conference on X-Ray Microscopy 1365 (2011), S. 69 
[130] Conley R., Liu C., Qian J., Kewish C. M., Macrander A. T., Yan H., Kang H. C., Maser J., Stephenson G. B.: Wedged multilayer Laue lens. Rev. Sci. Instrum. 79 (2008), S. 053104

[131] Chrisey D. B., Hubler G. K.: Pulsed Laser Deposition of Thin Films. John Wiley \& Sons, Inc. 1994

[132] Fähler S., Krebs H.-U.: Calculations and experiments of material removal and kinetic energy during pulsed laser ablation of metals. Appl. Surf. Sci. 96 (1996), S. 61

[133] Fähler S., Sturm K., Krebs H.-U.: Resputtering during the growth of pulsed-laserdeposited metallic films in vacuum and in an ambient gas. Appl. Phys. Lett. 75 (1999), S. 3768

[134] Fähler S., Kahl S., Weisheit M., Sturm K., Krebs H.-U.: The interface of laser deposited Cu/Ag multilayers: evidence of the "subsurface growth mode" during pulsed laser deposition. Appl. Surf. Sci. 154-155 (2000), S. 419

[135] Fähler S., StÖrmer M., Krebs H.-U.: Origin and avoidance of droplets during laser ablation of metals. Appl. Surf. Sci. 109/110 (1997), S. 433

[136] Fetzer D.: Homogenisierung der Schichtdicken laserdeponierter Filme. Bachelorarbeit, Institut für Materialphysik, Georg-August-Universität Göttingen, 2009

[137] Giannuzzi L. A., Stevie F. A.: Introduction To Focused Ion Beams. Springer Science + Business Media, Inc., Boston 2005

[138] Volkert C. A., Minor A. M.: Focused ion beam microscopy and micromachining. MRS Bulletin 32 (2007), S. 389

[139] Berglund M., Rymell L., Peuker M., Wilhein T., Hertz H. M.: Compact waterwindow transmission X-ray microscopy. Journal of Microscopy 197 (2000), S. 268

[140] Bayer A., Barkusky F., Döring S., Grossmann P., Mann K.: Applications of Compact Laser-Driven EUV/XUV Plasma Sources. X-Ray Opt. Instrum. 2010 (2010)

[141] Schwoerer H.: Generation of X-Rays by Intense Femtosecond Lasers. In: Dausinger F. (Hrsg.), Lubatschowski H. (Hrsg.), Lichtner F. (Hrsg.): Femtosecond Technology for Technical and Medical Applications Bd. 96. Springer-Verlag, Berlin, Heidelberg 2004 S. 235

[142] Fiedorowicz H., Bartnik A., Szczurek M., Daido H., Sakaya N., Kmetik V., Kato Y., Suzuki M., Matsumura M., Tajima J., Nakayama T., Wilhein T.: Investigation of soft X-ray emission from a gas puff target irradiated with a Nd:YAG laser. Opt. Comm. 163 (1999), S. 103 
[143] Salditt T., Kalbfleisch S., Osterhoff M., Krüger S. P., Bartels M., Giewekemeyer K., Neubauer H., Sprung M.: Partially coherent nano-focused x-ray radiation characterized by Talbot interferometry. Opt. Express 19 (2011), S. 9656

[144] Kalbfleisch S., Osterhoff M., Giewekemeyer K., Neubauer H., Küger S. P., Hartmann B., Bartels M., Sprung M., Leupold O., Siewert F., Salditt T.: The holography endstation of beamline P10 at PETRA III. AIP Conf. Proc.: The 10th International Conference On Radiation Instrumentation 1234 (2010), S. 433

[145] SAuerbrey G.: Verwendung von Schwingquarzen zur Wägung dünner Schichten und zur Mikrowägung. Zeitschrift für Physik A 155 (1959), S. 206

[146] Saxena A. M., Schoenborn B. P.: Multilayer neutron monochromators. Acta Crystallographica A 33 (1977), S. 805

[147] Windt D. L.: IMD-Software for modeling the optical properties of multilayer films. Computers in Physics 12 (1998), S. 360

[148] Parratt L. G.: Surface Studies of Solids by Total Reflection of X-Rays. Phys. Rev. 95 (1954), S. 359

[149] LiESE T.: Wachstum und Grenzflächenrauigkeit laserdeponierter $\mathrm{MgO} / \mathrm{Ti}$ - und $\mathrm{ZrO}_{2} /$ Ti-Multischichtsysteme. Diplomarbeit, Institut für Materialphysik, GeorgAugust-Universität Göttingen, 2007

[150] Hafner Bob: Introductory Transmission Electron Microscopy Primer. Characterization Facility, University of Minnesota-Twin Cities (2008), S. 1

[151] Hafner Bob: Scanning Electron Microscopy Primer. Characterization Facility, University of Minnesota-Twin Cities (2007), S. 1

[152] Spiess L., Schwarzer R., Behnken H., Teichert G.: Moderne Röntgenbeugung. Teubner Verlag 2005

[153] Banyay M., JuschKin L.: Table-top reflectometer in the extreme ultraviolet for surface sensitive analysis. Appl. Phys. Lett. 94 (2009), S. 063507

[154] National Instruments Corporation: Erste Schritte mit LabVIEW. Labview $^{\text {TM }} 2009$ Benutzerhandbuch (2009)

[155] Krebs H.-U., Bremert O.: Pulsed laser deposition of thin metallic alloys. Appl. Phys. Lett. 62 (1993), S. 2341

[156] Usoskin A., Garcìa-Moreno F., Freyhardt H. C., Knoke J., Sievers S., Gorkhover L., Hofmann A., PInk F.: Variable-azimuth laser ablation: principles and application for film deposition on long tubes and tapes. Appl. Phys. A 69 (1999), S. S423 
[157] Reese M., Schäfer B., Grossmann P., Bayer A., Mann K., Liese T., Krebs H.U.: Submicron focusing of XUV radiation from a laser plasma source using a multilayer Laue lens. Appl. Phys. A: Materials Science \& Processing 102 (2011), S. 85

[158] Schäfers F., Mertins H.-C., Schmolla F., Packe I., Salashchenko N. N., Shamov E. A.: Cr/Sc Multilayers for the Soft-X-Ray Range. Appl. Opt. 37 (1998), S. 719

[159] Kuhlmann T., Yulin S., Feigl T., Kaiser N., Gorelik T., Kaiser U., Richter W.: Chromium-Scandium Multilayer Mirrors for the Nitrogen $K_{\alpha}$ Line in the Water Window Region. Appl. Opt. 41 (2002), S. 2048

[160] Stollberg H., Yulin S., Takman P. A. C., Hertz H. M.: High-reflectivity Cr/Sc multilayer condenser for compact soft x-ray microscopy. Rev. Sci. Instrum. 77 (2006), S. 123101

[161] Fuhse C., Krebs H.-U., Vitta S., Johansson G. A.: Interface Quality and Thermal Stability of Laser-Deposited Metal/MgO Multilayers. Appl. Opt. 43 (2004), S. 6265

[162] REese M.: Charakterisierung von Optiken für weiche Röntgenstrahlung an Laborund Synchrotronstrahlungsquellen. Dissertation, Laser-Laboratorium Göttingen e. V., Georg-August-Universität Göttingen, 2011

[163] Singh R. K.: Transient plasma shielding effects during pulsed laser ablation of materials. J. Electron. Mater. 25 (1996), S. 125

[164] Kelly R., Cuomo J. J., Leary P. A., Rothenberg J. E., Braren B. E., Aliotta C. F.: Laser sputtering: Part I. On the existence of rapid laser sputtering at $193 \mathrm{~nm}$. Nucl Instrum Methods B: Beam Interactions with Materials and Atoms 9 (1985), S. 329

[165] Heiroth S., Koch J., Lippert T., Wokaun A., Günther D., Garrelie F., GuilLERMIN M.: Laser ablation characteristics of yttria-doped zirconia in the nanosecond and femtosecond regimes. J. Appl. Phys. 107 (2010), S. 014908

[166] Kelly R., Miotello A.: Mechanisms of Pulsed Laser Sputtering. In: Chrisey D. B. (Hrsg.), Hubler G. K. (Hrsg.): Pulsed Laser Deposition of Thin Films. John Wiley \& Sons, Inc. 1994 S. 55

[167] Voevodin A. A., Jones J. G., Zabinski J. S.: Characterization of $\mathrm{ZrO}_{2} / \mathrm{Y}_{2} \mathrm{O}_{3}$ laser ablation plasma in vacuum, oxygen, and argon environments. J. Appl. Phys. 88 (2000), S. 1088 
[168] Heiroth S., Lippert T., Wokaun A., Döbeli M., Rupp J. L. M., Scherrer B., GaucKler L. J.: Yttria-stabilized zirconia thin films by pulsed laser deposition: Microstructural and compositional control. J. Eur. Cer. Soc. 30 (2010), S. 489

[169] Hidalgo H., Reguzina E., Millon E., Thomann A.-L., Mathias J., BoulmerLeborgne C., Sauvage T., Brault P.: Yttria-stabilized zirconia thin films deposited by pulsed-laser deposition and magnetron sputtering. Surf. Coat. Tech. 205 (2011), S. 4495

[170] RöDer J., Krebs H.-U.: Frequency dependent smoothing of rough surfaces by laser deposition of $\mathrm{ZrO}_{2}$. Appl. Phys. A 90 (2008), S. 609

[171] RÖDer J., Liese T., Krebs H.-U.: Material-dependent smoothing of periodic rippled structures by pulsed laser deposition. J. Appl. Phys. 107 (2010), S. 103515

[172] Voevodin A. A., Jones J. G., Zabinski J. S.: Structure control of pulsed laser deposited $\mathrm{ZrO}_{2} / \mathrm{Y}_{2} \mathrm{O}_{3}$ films. J. Vac. Sci. Technol. A 19 (2001), S. 1320

[173] Okabayashi J., Toyoda S., Kumigashira H., Oshima M., Usuda K., Niwa M., Liu G. L.: Crystallization and chemical structures with annealing in $\mathrm{ZrO}_{2}$ gate insulators studied by photoemission spectroscopy and x-ray absorption spectroscopy. J. Vac. Sci. Technol. A 23 (2005), S. 1554

[174] Liese T., Radisch V., Krebs H.-U.: Fabrication of multilayer Laue lenses by a combination of pulsed laser deposition and focused ion beam. Rev. Sci. Instrum. 81 (2010), S. 073710

[175] Giannuzzi L. A., Prenitzer B. I., Kempshall B. W.: Ion-Solid Interactions. In: Giannuzzi L. A. (Hrsg.), Stevie F. A. (Hrsg.): Introduction To Focused Ion Beams. Springer Science + Business Media, Inc., Boston 2005 S. 13

[176] Liese T., Radisch V., Knorr I., Reese M., Grossmann P., Mann K., Krebs H.-U.: Development of laser deposited multilayer zone plate structures for soft X-ray radiation. Appl. Surf. Sci. 257 (2011), S. 5138

[177] Alda J.: Laser and Gaussian Beam Propagation and Transformation. Opt. Eng. (2003), S. 999

[178] Eichler J., Dünkel L., Eppich B.: Die Strahlqualität von Lasern - Wie bestimmt man Beugungsmaßzahl und Strahldurchmesser in der Praxis? Laser Technik Journal 1 (2004), S. 63

[179] International Organisation for Standardization, Genf, Schweiz. ISO 11146: Laser and laser-related equipment - Test methods for laser beam parameters - Beam width, divergence angle and beam propagation factor, (1999) 
[180] Windischmann H.: An intrinsic stress scaling law for polycrystalline thin films prepared by ion beam sputtering. J. Appl. Phys. 62 (1987), S. 1800

[181] D'Heurle F. M., Harper J. M. E.: Note on the origin of intrinsic stresses in films deposited via evaporation and sputtering. Thin Solid Films 171 (1989), S. 81

[182] Li D., Guruvenket S., Hassani S., Bousser E., Azzi M., Szpunar J. A., KlemberG-SAPIeHA J. E.: Effect of Cr interlayer on the adhesion and corrosion enhancement of nanocomposite TiN-based coatings deposited on stainless steel 410. Thin Solid Films 519 (2011), S. 3128

[183] Pischow K. A., Eriksson L., Harju E., Korhonen A. S., Ristolainen E. O.: The influence of titanium interlayers on the adhesion of PVD TiN coatings on oxidized stainless steel substrates. Surf. Coat. Tech. 58 (1993), S. 163

[184] Mimura H., Handa S., Kimura T., Yumoto H., Yamakawa D., Yokoyama H., Matsuyama S., Inagaki K., Yamamura K., Sano Y., Tamasaku K., Nishino Y., Yabashi M., IshikaWA T., Yamauchi K.: Breaking the $10 \mathrm{~nm}$ barrier in hard-X-ray focusing. Nature Phys. 6 (2010), S. 122

[185] Ruhlandt A., Liese T., Radisch V., Krüger S. P., Osterhoff M., Giewekemeyer K., Krebs H.-U., Salditt T.: A combined Kirkpatrick-Baez mirror and multilayer lens for sub-10 nm x-ray focusing. AIP Advances 2 (2012), S. 012175

[186] Windt D. L., Donguy S., Hailey C. J., Koglin J., Honkimaki V., Ziegler E., Christensen F. E., Chen H., Harrison F. A., Craig W. W.: W/SiC X-Ray multilayers optimized for use above $100 \mathrm{keV}$. Appl. Opt. 42 (2003), S. 2415

[187] Ruhlandt A.: Design und Berechnung eines kohärenten sub-20nm Fokus für das Strahlrohr P10 an Petra III. Bachelorarbeit, Institut für Röntgenphysik, Georg-AugustUniversität Göttingen, 2011

[188] Massalksi T.-B.: Binary Alloy Phase Diagrams, 2nd Edition, Vol. 3. ASM International 1990

[189] Chen L. J.: Solid state amorphization in metal/Si systems. Mat. Sci. Eng. R 29 (2000), S. 115

[190] von Allmen M.: Laser Beam Interactions with Materials Physical Principles and Applications. Springer-Verlag, Berlin, Heidelberg, New York 1987, Kap. 5

[191] Störmer M., Sturm K., Fähler S., Weisheit M., Winkler J., Kahl S., Kesten P., Pundt A., Seibt M., Senz S., Krebs H.-U.: Study of laser-deposited metallic thin films by a combination of high-resolution ex-situ and time-resolved in-situ experiments. Appl. Phys. A 69 (1999), S. S455 
[192] Sturm K., Krebs H.-U.: Quantification of resputtering during pulsed laser deposition. J. Appl. Phys. 90 (2001), S. 1061

[193] Biswas A., Bhattacharyya D.: Correlation of interface roughness for ion beam sputter deposited W/Si multilayers. J. Appl. Phys. 109 (2011), S. 084311

[194] Windt D. L., Christensen F. E., Craig W. W., Hailey C., Harrison F. A., Jimenez-Garate M., Kalyanaraman R., Mao P. H.: Growth, structure, and performance of depth-graded W/Si multilayers for hard x-ray optics. J. Appl. Phys. 88 (2000), S. 460

[195] Haefer R. A.: Oberflächen- und Dünnschicht-Technologie, Teil I - Beschichtung von Oberflächen. Springer-Verlag, Berlin, Heidelberg, New York 1987, Kap. 6

[196] Yamamura Y., Tawara H.: Energy Dependence Of Ion-Induced Sputtering Yields From Monatomic Solids At Normal Incidence. Atomic Data and Nuclear Data Tables 62 (1996), S. 149

[197] Ziegler J. F., Biersack J. P., Ziegler M. D.: The Stopping and Range of Ions in Matter. SRIM Co. 2008

[198] Möller W., Eckstein W.: Tridyn - A TRIM simulation code including dynamic composition changes. Nucl. Instrum. Meth. Phys. Res. B 2 (1984), S. 814

[199] Berg S., Katardjiev I.: Resputtering effects during ion beam assisted deposition and the sputter yield amplification effect. Surf. Coat. Tech. 84 (1996), S. 353

[200] Berg S., Barklund A. M., Gelin B., Nender C., Katardjiev I.: Atom assisted sputtering yield amplification. J. Vac. Sci. Technol. A 10 (1992), S. 1592

[201] Nender C., Katardjiev I. V., Biersack J. P., Berg S., Barklund A. M.: Numerical and experimental studies of the sputter yield amplification effect. Radiation Effects and Defects in Solids 130 (1994), S. 281

[202] Berg S., Katardjiev I. V.: Preferential sputtering effects in thin film processing. J. Vac. Sci. Technol. A 17 (1999), S. 1916

[203] Austgen M., Koehl D., Zalden P., Kubart T., Nyberg T., Pflug A., Siemers M., Berg S., Wuttig M.: Sputter yield amplification by tungsten doping of $\mathrm{Al}_{2} \mathrm{O}_{3}$ employing reactive serial co-sputtering: process characteristics and resulting film properties. J. Phys. D 44 (2011), S. 345501

[204] Krüger S. P., Giewekemeyer K., Kalbfleisch S., Bartels M., Neubauer H., SALDITT T.: Sub-15 nm beam confinement by two crossed $x$-ray waveguides. Opt. Express 18 (2010), S. 13492 
[205] Vaziri M. R. R., Hajiesmaeilbaigi F., Maleki M. H.: Monte Carlo simulation of the subsurface growth mode during pulsed laser deposition. J. Appl. Phys. 110 (2011), S. 043304 


\section{Danksagung}

Zum Abschluss möchte ich all denen meinen herzlichsten Dank aussprechen, ohne die es weder einen Abschluss noch einen Anfang dieser Arbeit gegeben hätte.

Allen voran bedanke ich mich bei Prof. Dr. Hans-Ulrich Krebs für die überaus exzellente Betreuung und die phantastische Atmosphäre während meiner Promotion. Nicht nur eine fest entschlossene Begeisterung an der Physik konnte er mir tagtäglich vermitteln, sondern auch eine außergewöhnliche Beobachtungsgabe physikalischer und menschlicher Natur, für die ich ihm genau genommen noch dankbarer bin. Viele gemeinsam verbrachte „Abenteuer“, sei es die Röntgenmikroskopie-Tagung in Chicago oder die Fahrradtour nach Hemeln, werden mir lange in Erinnerung bleiben.

Ganz besonders bedanken möchte ich mich bei meiner Arbeitsgruppe für das großartige freundschaftliche und kollegiale Verhältnis: Susanne Schlenkrich für die vielen tollen Gespräche, die längst nicht nur Physik beinhalteten, und natürlich für ihre unbändige gute Laune, die sehr ansteckend sein kann. Felix Schlenkrich für eine super Zeit bei allen möglichen Diskussionen und Gesprächen sowie für das gegenseitige Pushen bei zahlreichen Jogging-Touren und Kicker-Runden. Weiterhin bin ich dankbar, solch interessante Persönlichkeiten wie Sarah Hoffmann und Benedikt Ernst kennengelernt zu haben, die mich mit ihren Arbeiten an $\mathrm{Ti} / \mathrm{ZrO}_{2}-$ Schichten begleitet haben. Meinem „Nachfolger" Christian Eberl danke ich für seine enthusiastische Arbeitsweise, die mit der gemeinsamen Herstellung äußerst schwer herzustellender W-Schichten begonnen hat. Ich freue mich, dass er zusammen mit Florian Döring auf dem Gebiet der Transmissionsoptiken weiterarbeitet. Ebenfalls wünsche ich den jüngsten Mitgliedern Sara-Lisa Jäckle und Stephanie Demuth eine angenehme und erfolgreiche Zeit in unserer Arbeitsgruppe.

Auch bei ehemaligen Gruppenmitgliedern möchte ich mich bedanken: Dr. Andreas Meschede für zahlreiche physikalische Denkanstöße und die einführende Unterstützung in LabVIEW sowie Dr. Johanna Röder für die stete Hilfsbereitschaft während und nach ihres Studiums, die allesamt zum Gelingen dieser Arbeit beigetragen haben. Dirk 
Fetzer danke ich für die tolle Zeit während der Betreuung seiner Bachelor-Arbeit, in der wir gemeinsam an der Kammer rumgeschraubt und programmiert und uns über jeden Schrittmotor gefreut haben, der sich endlich drehen wollte.

Ganz herzlich möchte ich mich bei unserem FIB-Experten Volker Radisch bedanken, ohne den vermutlich keine einzige in dieser Arbeit gezeigte MLL zustande gekommen wäre. Nur durch seine Erfahrung und sein Interesse an unseren Ideen, mit fokussierten Ionenstrahlen die unterschiedlichsten Röntgenlinsen zu schnitzen, konnten diese auch tatsächlich umgesetzt werden. Und ich danke ihm besonders dafür, dass er an einem sonnigen Samstagnachmittag, als ich während einer FIB-Session am Ende meiner Möglichkeiten angekommen war, extra in die Uni kam um mir unter die Arme zu greifen. Natürlich danke ich auch Dr. Peter Wilbrandt für die theoretische FIB-Einführung.

Von der technischen Seite her danke ich außerdem Conni Mewes und Michael Malchow, die mir stets mit Rat und Tat zur Seite standen. Bedanken möchte ich mich auch bei den Feinmechanik- und Elektronik-Werkstätten, welche die Umsetzung sämtlicher Änderungen an der Depositionsanlage maßgeblich unterstützt haben. Inga Knorr, Matthias Hahn und Burkhard Roos danke ich für die Anfertigung der TEMAufnahmen. Mein Dank gilt außerdem allen anderen Mitarbeitern des Instituts, die das angenehme Arbeitsklima abgerundet haben.

Meinen herzlichen Dank möchte ich der Arbeitsgruppe „Optik/Kurze Wellenlängen“ des Laser-Laboratoriums Göttingen e. V. um Dr. Klaus Mann für die grundlegende Zusammenarbeit im gemeinsamen SFB-Projekt C4 entgegenbringen. Für die MLLFokusexperimente und die EUV-Reflektometriemessung sowie die Übermittlung theoretischen Wissens rund um lasergetriebene Plasmaquellen bedanke ich mich bei Dr. Christian Peth, Dr. Frank Barkusky, Dr. Michael Reese, Peter Großmann und Stefan Döring.

Ich danke den Mitarbeitern des Instituts für Röntgenphysik für die gute Zusammenarbeit an Multilagenoptiken für harte Röntgenstrahlung. Vor allem Prof. Dr. Tim Salditt, der mit seinen nicht enden wollenden Ideen zur Nanofokussierung von Röntgenstrahlung einen wesentlichen Einfluss auf den Verlauf meiner Arbeit genommen hat. Für viele hilfreiche Diskussionen und die MLL-Untersuchung am DESY im 24-StundenBetrieb bin ich Dr. Sven Philip Krüger, Dr. Klaus Giewekemeyer, Dr. Markus Osterhoff und Aike Ruhlandt sehr dankbar. Dr. Dong-Du Mai danke ich für ein stets offenes Ohr und die zahlreichen Versuche, einige Optiken im „table-top“-Röntgenmikroskop zu untersuchen. 
Dem SFB 755 „Nanoscale Photonic Imaging“ danke ich für die finanzielle Unterstützung und die herrliche Atmosphäre während sämtlicher Herbst- und Winterschulen, bei denen es zusammen mit Eva Hetzel und Kerstin Grüny immer etwas zu lachen gab. Ihnen bin ich neben den vielen netten Gesprächen außerdem sehr dankbar, mir im bürokratischen Labyrinth den richtigen Weg gezeigt zu haben.

Prof. Dr. Hans Hofsäß danke ich für die fruchtbaren Diskussionen rund um SRIMund TRIDYN-Simulationen zum Verständnis des W/Si-Schichtwachstums. Weiterhin möchte ich mich herzlich bei Prof. Dr. Sarah Köster für die Übernahme des Korreferats bedanken.

Ich danke allen meinen Freunden, die mich während der gesamten Studienzeit begleitet und diese angenehm verschönert haben. Ohne den prägenden Zusammenhalt wäre ich sicherlich nicht so weit gekommen.

Mein herzlichster Dank gilt natürlich meiner Familie für die langjährige Unterstützung in allen erdenklichen Lebenslagen und für die als nicht selbstverständlich gegebene Möglichkeit, überhaupt studieren zu können. Besonders möchte ich mich bei Steffi für ihre Warmherzigkeit und ihre Liebe bedanken, die sie mir in der wenigen Zeit zu zweit unaufhörlich entgegenbringt. Ich könnte mir kein besseres Mittel vorstellen, die Physik Physik sein zu lassen. 



\section{Lebenslauf}

Angaben zur Person

Name:

Geburtsdatum:

Geburtsort:

Staatsangehörigkeit:

Familienstand:

\section{Schulbildung}

1988 - 1992

$1992-2001$

2001

\section{Wehrdienst}

2001 - 2002

\section{Studium}

$10.2002-04.2005$

20.04.2005

$04.2005-01.2008$

29.01.2008

02.2008
Tobias Liese

02.04.1982

Rotenburg a.d. Fulda

deutsch

ledig

Grundschule, Sontra-Ulfen

Gesamtschule mit gymnasialer Oberstufe, Adam-von-TrottSchule Sontra

Abitur

Grundwehrdienst im Panzeraufklärungsbataillon 1./13, Gotha

Grundstudium in Physik an der Georg-August-Universität Göttingen

Vordiplom in Physik

Hauptstudium in Physik an der Georg-August-Universität Göttingen

Diplom in Physik, Thema der Diplomarbeit: „Wachstum und Grenzflächenrauigkeit laserdeponierter $\mathrm{MgO} / \mathrm{Ti}$ - und $\mathrm{ZrO}_{2} /$ Ti-Multischichtsysteme", angefertigt am Institut für Materialphysik in der Arbeitsgruppe von Prof. Dr. H.-U. Krebs Beginn der Promotion am Institut für Materialphysik in der Arbeitsgruppe von Prof. Dr. H.-U. Krebs, wissenschaftlicher Mitarbeiter im Sonderforschungsbereich 755 „Nanoscale Photonic Imaging“" 